\title{
Omega Minus \\ Polarization and Magnetic Moment
}

HERMAN THOMAS DIEHL, III

May, 1990

Supported in part by:

National Science Foundation

Department of Physics and Astronomy 


\section{Omega Minus Polarization and Magnetic Moment}

by Herman Thomas DiehI, III

A dissertation submitted to the

Graduate School- New Brunswick

Rutgers, The State University of New Jersey

in partial fulfilment of the requirements

for the degree of

Doctor of Philosophy

Graduate Program in Physics

Written under the direction of

Dr. Gordon B. Thomson

and approved by
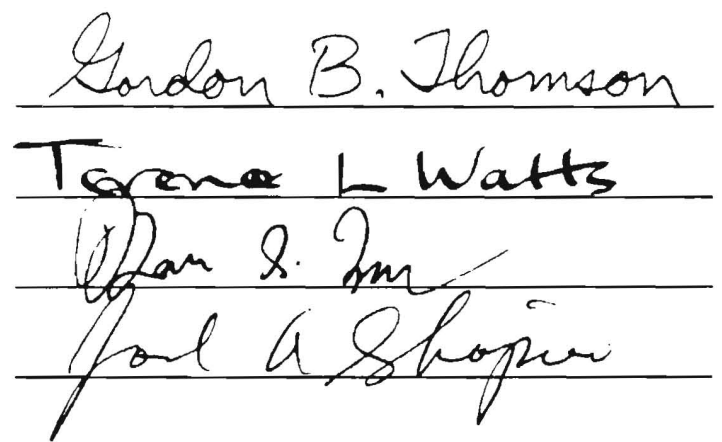

New Brunswick, New Jersey

May, 1990 


\title{
ABSTRACT OF THE DISSERTATION
}

\section{Omega Minus Polarization and Magnetic Moment}

\author{
by Herman Thomas Diehl, III, Ph.D. \\ Dissertation Director: Dr. Gordon B. Thomson
}

We have studied the polarization of a secondary beam of $\Omega^{-} \mathrm{s}$ and $\Xi^{-} \mathrm{s}$ produced by 800 $\mathrm{GeV} / \mathrm{c}$ unpolarized protons. The polarization of a sample of $\Omega^{-} \mathrm{s}$ in the kinematic region $x_{f}=0.42-0.60$ and $P_{t}=0.8-1.2 \mathrm{GeV} / \mathrm{c}$ containing $88 \mathrm{~K}$ events was $-0.018 \pm 0.09$. The polarization of a sample containing $663 \mathrm{~K} \Xi^{-} \mathrm{s}$ in the same kinematic region was $-0.109 \pm 0.004$.

A tertiary beam containing polarized $\Omega^{-} \mathrm{s}$ and $\Xi^{-} \mathrm{s}$ was produced by a secondary beam which contained polarized neutral hyperons. This spin-transfer method produced an $\Omega^{-}$polarization of $-0.069 \pm 0.022$. The $\Xi^{-}$polarization averaged $-0.112 \pm 0.005$ and increased with the $\Xi^{-}$momentum.

The magnetic moment of the $\Omega^{-}$and $\Xi^{-}$hyperons were measured via the spinprecession technique. $\mu_{\Omega^{-}}=-2.02 \pm 0.16 \pm 0.12 \mu_{N} \cdot \mu_{\Xi-}=-0.650 \pm 0.005 \pm 0.002 \mu_{N}$, where the first uncertainty is statistical and the second systematic. 


\section{Acknowledgements}

I would like to thank Gordon Thomson for introducing me to experimental high energy physics and for his tolerant and continuous support as my thesis advisor.

Many current and ex-members of E756 and its predecessors contributed to this experiment. Kam-Biu Luk was an inexhaustable source of ideas, and his committment and devotion to the experiment and analysis were invaluable. Gina Rameika's efforts in bringing up the beamline in Phase 1 and Phase 2 and her enthusiasm during the analysis were appreciated. In addition, I particularly stress the importance of discussions, explanations and criticisms from: Ken Heller, Tom Devlin, Ken Johns, Cat James, Scott Teige, Pak-Ming Ho, Jeff Duryea, and Gerald "Hobbes" Guglielmo.

Above all, I would like to thank Brenna Flaugher, my parents, my brother and my sister for the understanding, support, patience and encouragement they gave me throughout my years as a student.

This experiment would not have been possible without the cooperation and efforts of the Fermilab staff. 


\section{Table of Contents}

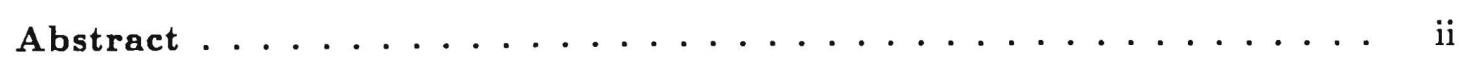

Acknowledgements $\ldots \ldots \ldots \ldots \ldots \ldots \ldots$ iii

List of Tables ...................... . . . . .

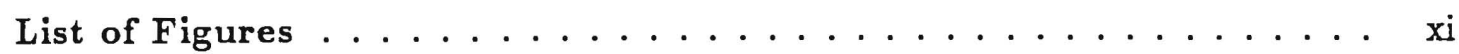

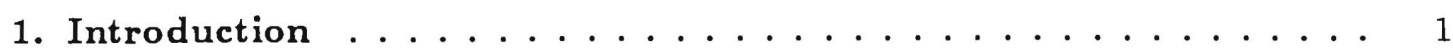

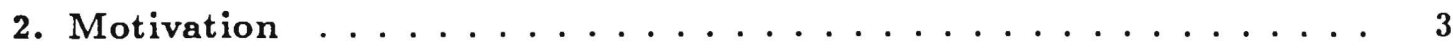

2.1. Experimental Background . . . . . . . . . . . . . 3

2.2. Omega Minus Properties .................. 6

2.3. Theoretical Background . . . . . . . . . . . . . 7

2.4. Magnetic Moment Calculations ............... 8

2.5. Polarization Theories . . . . . . . . . . . . . . . 12

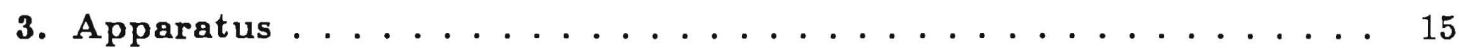

3.1. Beamline and Target Area Enclosure . . . . . . . . . . . . . 15

3.2. Hyperon Magnet and Charged Particle Channel . . . . . . . . . 18

3.3. Spectrometer ....................... 22

3.4. Trigger ............................ 27

3.5. Data Acquisition . . . . . . . . . . . . . . . . 29

3.6. Rates and Tapes ..................... 30

4. Event Analysis ...................... 37

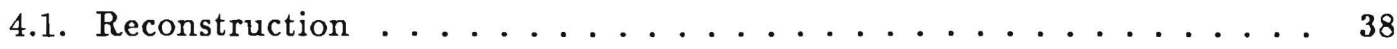

4.2. Monte Carlo. . . . . . . . . . . . . . . . . . 46

4.3. Omega Minus Event Selection and Background . . . . . . . . 56 
4.4. Cascade Minus Event Selection and Background . . . . . . . . 73

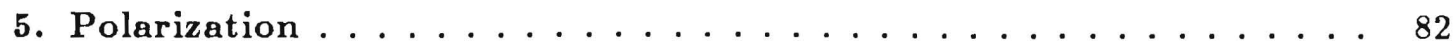

5.1. The Joint Angular Decay Distribution and Polarization . . . . . . . 82

5.2. The Hybrid Monte Carlo . . . . . . . . . . . . . . . . 88

5.3. Phase 1 Omega Minus Polarization Results . . . . . . . . . . . 90

5.4. Phase 1 Cascade Minus Polarization Results . . . . . . . . . . . 101

5.5. Phase 2 Omega Minus Polarization Results ........... 107

5.6. Phase 2 Cascade Minus Polarization Results . . . . . . . . . . . 115

5.7. Monte Carlo Studies . . . . . . . . . . . . . . . . . . . . 124

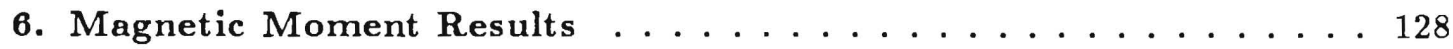

6.1. Cascade Minus Magnetic Moment Result . . . . . . . . . . . . 128

6.2. Omega Minus Magnetic Moment Result . . . . . . . . . . . . . 131

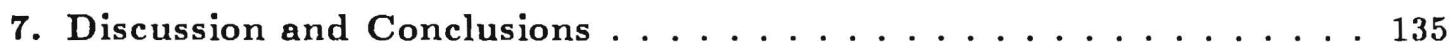

Appendix A. Spin Precession in a Magnetic Field . . . . . . . 137

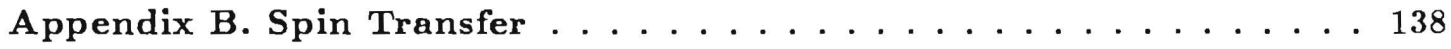

B.1. Neutral Particle Cross-sections . . . . . . . . . . . . 138

B.2. Neutral Beam Polarization . . . . . . . . . . . . . 143

B.3. Omega Minus and Cascade Minus Production from the Neutral Beam . 144

B.4. Spin Transfer to the Omega Minus and Cascade Minus . . . . . . 145

B.5. Phase 2 Polarization Predictions . . . . . . . . . . . 149

B.6. Discussion . . . . . . . . . . . . . . . 149

Appendix C. The Rutgers $1 \mathrm{~mm}$ Pitch MWPCs . . . . . . . . 152

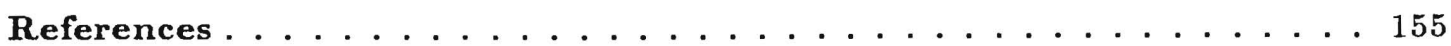

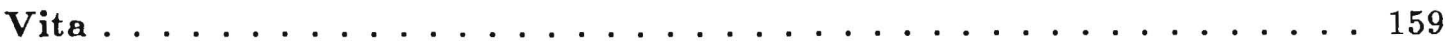




\section{List of Tables}

2.1. Magnetic moment measurements. Before E756. The nuclear magneton

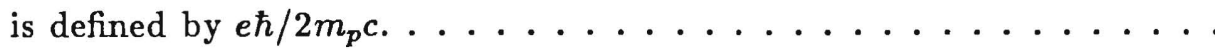

2.2. Naive quark model wavefunctions. These are simplified wavefunctions presented for the purpose of illustrating the baryon's flavor and spin content. To obtain the complete wavefunctions, it is necessary to permute the quark flavors and multiply by the anti-symmetric color singlet. . .

2.3. Naive quark model (without corrections) magnetic moment predictions. The numerical values come from using the $p, n$, and $\Lambda$ wavefunctions to determine the up, down and strange quark magnetic moments. Here, $\mu_{u}$ $=1.8516, \mu_{d}=-0.97184$ and $\mu_{s}=-0.613 \pm 0.004$ nuclear magnetons. $\ldots 10$

2.4. Theoretical predictions of the $\Omega^{-}$magnetic moment. . . . . . . . 12

3.1. Hyperon Magnet field integral vs. current. . . . . . . . . . . . . 22

3.2. MWPC construction characteristics. Cathode refers to the high voltage wires; anode refers to the sense wires. The tension is given in terms of the weight applied to each wire. $(1 \mathrm{mil}=0.001$ inch $=25.4$ microns. $)$.

3.3. MWPC characteristics. The location is the distance from the collimator exit of the center of the chamber along $\hat{z}$. The efficiency is the average of the $\mathrm{x}$ and $\mathrm{y}$ planes over the whole data sample. . . . . . . .

3.4. Phase 1. Typical rates for each beam condition. Main conditions. The rates/proton for target out conditions are typically 50 times lower. . . . 31

3.5. Phase 2. Typical rates. . . . . . . . . . . . . . 32 
3.6. Phase 1. Number of tapes for each beam condition. Main conditions. There were $\sim 55.5 \mathrm{M}$ (million) 3-trk triggers recorded among these conditions. ............................... 34

3.7. Phase 1. Number of tapes for each beam condition. Odd conditions. The target in or target out labels apply to all the conditions within their respective boxes. There were $\sim 11.0 \mathrm{M}$ 3-trk triggers recorded among these conditions. . . . . . . . . . . . . . . 35

3.8. Phase 2. Number of tapes for each beam condition. There were $\sim 12.7$ M 3-trk triggers recorded among these conditions. . . . . . . . . 36

4.1. Reconstruction catagories. Only $\mathrm{IOK}=0$ events passed the reconstruction algorithm and were classified "good" three track events. These are compiled from the whole data sample. . . . . . . . . . . . . . 42

4.2. Summary of the cuts used to select $\Omega^{-}$s. A cut number is assigned to each cut for use in Table 4.3. Cuts 0 and 1 were made while processing the raw data tapes as described in Section 4.1. The remainder were applied to the "tapout" tapes. . . . . . . . . . . . 59

4.3. Summary of the cuts used to select $\Omega^{-}$s. The first column is the data. The next 3 columns are Monte Carlo events scaled so that the bottom and top lines equal the number of data events. The Monte Carlo events were generated with the Hyperon Magnet at -450 amps. . . . . . . . 61

4.4. Phase 1. Number of $\Omega^{-}$s for each beam condition. Main conditions. . . 70

4.5. Phase 1. Number of $\Omega^{-}$s for each beam condition. Odd conditions. . . 71

4.6. Phase 2. Number of $\Omega^{-}$s for each beam condition. . . . . . . . . 72

4.7. Summary of the cuts used to select the $\Xi^{-}$event sample. Including Phase 1 and Phase 2, there were an estimated $13.6 \mathrm{M} \Xi^{-}$events which passed

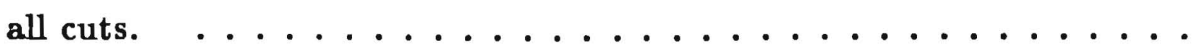


4.8. Summary of cuts used to select $\Xi^{-}$s. The $\Xi^{-}$sample was scaled to the estimated number of data $\Xi^{-} \rightarrow \Lambda \pi^{-}$. The $\Omega^{-}$sample was scaled to the estimated number of data $\Omega^{-} \rightarrow \Lambda \mathrm{K}^{-} \ldots \ldots \ldots 75$

4.9. Phase 1. Number of $\Xi^{-} \mathrm{s}$ for each beam condition. This is a sample of $\sim 8 \%$ of the available $\Xi^{-} \ldots \ldots \ldots \ldots$

4.10. Phase 2. Number of $\Xi^{-} \mathrm{s}$ for each beam condition. The Hyperon Magnet $=-450$ Amps sample is about $12 \%$ of all the $\Xi^{-}$available at that

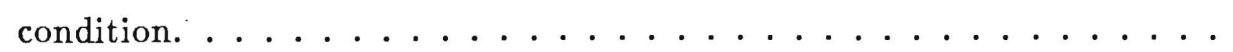

5.1. Phase 1. $\Omega^{-}$asymmetry results from the Hybrid Monte Carlo. These were used in Equations 5.25 and $5.26 . \ldots \ldots \ldots \ldots$

5.2. Phase 1. Polarization results at $\pm 2.5 \mathrm{mR}$ targeting angle for the $\Omega^{-}$data from the Hybrid Monte Carlo. It is assumed that $\gamma_{\Omega^{-}}=1$. Otherwise, the polarizations and uncertainties should be scaled by $-5 / 3$. . . . .

5.3. Phase 1. Bias results at $\pm 2.5 \mathrm{mR}$ targeting angle for the $\Omega^{-}$data from the Hybrid Monte Carlo. . . . . . . . . . . . . . . .

5.4. Phase 1. Statistics of the $\Omega^{-}$polarization results from the Hybrid Monte Carlo.

5.5. Phase $1 \Omega^{-}$grand fit results. The polarization is presented $\gamma$ is +1 . The polarization and error bar are multiplied by $-5 / 3$ if $\gamma$ is -1 . The last two columns are the change in polarization when $\mathrm{g}$ is changed by $\pm 1 \sigma$ (e.g.

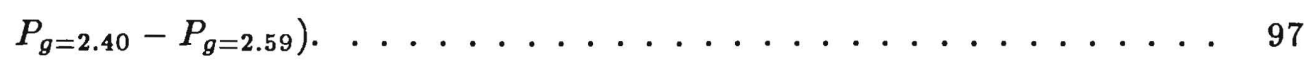

5.6. Phase $1 \Omega^{-}$grand fit bias results. . . . . . . . . . . . 99

5.7. Phase 1. $\Xi^{-}$asymmetry results from the Hybrid Monte Carlo. These were used in Equations 5.25 and $5.26 \ldots \ldots \ldots 2 \ldots \ldots$. . . . . . . 102

5.8. Phase 1. Polarization results at $\pm 2.5 \mathrm{mR}$ targeting angle for the $\Xi^{-}$ data from the Hybrid Monte Carlo. . . . . . . . . . . . . . . 103 
5.9. Phase $1 . \Xi^{-}$polarization results at $\pm 2.5 \mathrm{mR}$ targeting angle. These are the initial polarizations at the $\Xi^{-}$production target. They are calculated

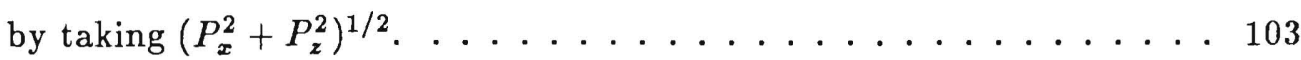

5.10. Phase 1 . Bias results at $\pm 2.5 \mathrm{mR}$ targeting angle for the $\Xi^{-}$data from the Hybrid Monte Carlo. . . . . . . . . . . . . . . . 105

5.11. Phase 1. Statistics of the $\Xi^{-}$polarization results from the Hybrid Monte

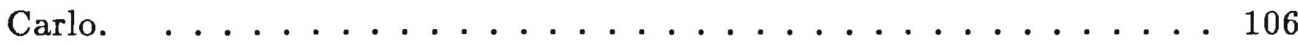

5.12. Phase 2. $\Omega^{-}$asymmetry results from the Hybrid Monte Carlo. These were used in Equations 5.25 and 5.26. The results from $0 \mathrm{mR}$ are given in Table $5.16 . \ldots \ldots \ldots \ldots \ldots$. . . . . . . . . . . . . . . . . . .

5.13. Phase 2. Polarization results at $\pm 2 \mathrm{mR}$ targeting angle for the $\Omega^{-}$ data. It is assumed that $\gamma_{\Omega^{-}}=+1$. If $\gamma_{\Omega}=-1$, the polarizations and uncertainties are scaled by $-5 / 3 . \ldots \ldots \ldots 110$

5.14. Phase 2. Polarization at the $\Omega^{-}$production target. These are calculated from the $P_{x}$ component of the polarization and the magnetic moment measured in the next chapter. $\gamma$ is assumed to be +1 . If it is -1 , all of the numbers should be multiplied by $-5 / 3 \ldots \ldots$. . . . . . . . . 111

5.15. Phase 2. Bias results at $\pm 2 \mathrm{mR}$ targeting angle for the $\Omega^{-}$data. $\ldots 112$

5.16. Phase 2. Bias results at $0 \mathrm{mR}$ targeting angle for the $\Omega^{-}$data. These are to be compared with Table $5.15 \ldots \ldots \ldots 112$

5.17. Phase 2. Statistics of the $\Omega^{-}$polarization results. The results are shown as a function of momentum bin for the $-450 \mathrm{Amp}$ sample. Only averages over all momentum are presented for the -1000 Amp sample because of the low number of events. . . . . . . . . . . . . . 113

5.18. Phase 2. $\Xi^{-}$asymmetry results from the Hybrid Monte Carlo. These were used in Equations 5.25 and 5.26. The results from $0 \mathrm{mR}$ are given in Table 5.22. . . . . . . . . . . . . . . . . . . . 119 
5.19. Phase 2. $\Xi^{-}$polarization results at $\pm 2 \mathrm{mR}$ targeting angle. These are the components of the polarization after precessing in the Hyperon magnet. . . . . . . . . . . . . . . 120

5.20. Phase $2 . \Xi^{-}$polarization results at $\pm 2 \mathrm{mR}$ targeting angle. These are the initial polarizations at the $\Xi^{-}$production target. They are calculated by taking $\left(P_{x}^{2}+P_{z}^{2}\right)^{1 / 2} \ldots \ldots \ldots \ldots \ldots \ldots \ldots \ldots \ldots \ldots \ldots \ldots \ldots \ldots$

5.21. Phase 2 . $\Xi^{-}$bias results at $\pm 2 \mathrm{mR}$ targeting angle. . . . . . . . 122

5.22. Phase $2 . \Xi^{-}$polarization (bias) results at $0 \mathrm{mR}$ targeting angle. These results are to be compared with the bias measured at $\pm 2 . \mathrm{mR}$, shown

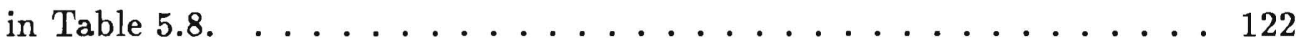

5.23. Phase 2. Statistics of the $\Xi^{-}$polarization results. . . . . . . . 123

5.24. Phase 2. Bias results from the combined $\pm 2 \mathrm{mR}$ targeting angle for the Monte Carlo $\Omega^{-}$data. These are to be compared with Table 5.13. . . . 124

5.25. Monte Carlo Study. Effect of Rank-3 Tensor Polarization. The contamination of vector polarization from a rank-3 tensor polarization which alternates $\operatorname{sign}$ with targeting angle is shown. $\ldots \ldots \ldots 127$

6.1. Phase 1 and Phase $2 . \Xi^{-}$precession angles. . . . . . . . . . 129

6.2. Phase $2 . \Omega^{-}$precession angles. . . . . . . . . . . 131

6.3. $\Omega^{-}$precession angle ambiguities. There are 4 degrees of freedom. These were determined by adding (or subtracting) factors of $\pi$ from the results of Table 6.2 and performing the straight-line fit through the origin to determine the magnetic moment. . . . . . . . . . . . . 133

B.1. These are the constants from the parameterization of the data to Equation B.1. ....................... 140

B.2. Polarization expectations of the D-M Model. The parameters $\epsilon$ and $\delta$ are roughly 0.10 to 0.30 . The incoming beam polarization is $d$. These estimates are given to first order in all of the parameters. . . . . . . 148 


\section{List of Figures}

2.1. Hyperon polarization from $400 \mathrm{GeV} / \mathrm{c}$ protons. The production angle was $5 \mathrm{mR}$ except for the $\bar{\Lambda}$ and $\Sigma^{-}$where the production angle was $7.5 \mathrm{mR}$. The target was Be. . . . . . . . . . . . 4

2.2. Lund model of polarization. A quark or diquark pair is created along a color force string. The transverse momentum of the quark or diquark pair results in an angular momentum which is compensated by it s spin.

3.1. The target enclosure, $\mathrm{PC} 3$, shown in vertical view for Phase 1 (upper) and Phase 2 (lower). The scales are different for $\mathrm{x}$ and $\mathrm{y}$. The size of the

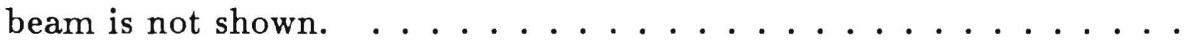

3.2. Phase 1 collimator. The shaded sections are made of tungsten. The unshaded regions are brass. The views from above (plan) and from the side (elevation) are shown, including the direction of the beam centerline. The magnetic field direction is shown. . . . . . . . . . . . .

3.3. Phase 2 collimators. The charged particle channel is the same one used in Phase 1. The plan and elevation views are shown. The shaded sections are made of tungsten. The unshaded regions are brass. The magnetic field directions are shown. . . . . . . . . . . . .

3.4. $\Xi^{-} P_{y}$ at the production target for Phase 1 and $P$ hase 2. Both positive and negative targeting argles are shown. These $\Xi^{-} \mathrm{s}$ were recorded with the Hyperon Magnet current set at -1000 Amps. . . . . . . . . . 
3.5. Relative charged particle collimator acceptance. This plot shows the acceptance of beam pions for the seven Hyperon Magnet fields used in the experiment. This was determined from a Monte Carlo simulation of

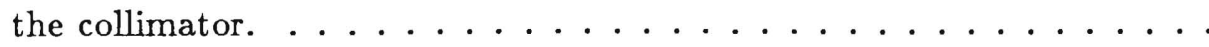

3.6. Spectrometer. The spectrometer is shown in the vertical view. The path of a typical $\Omega^{-}$and it's decay products are shown. Solid lines represent the charged tracks. The dashed line represents the $\Lambda$. . . . . .

3.7. Field of the BM109s. The plots show $B_{y}, B_{x}$, and $B_{z}$, respectively, of both BM109s. The horizontal scale is distance along the center of the magnets in $\mathrm{cm}$. The vertical scale is the field in Kgauss. The right hand side is the $\mathrm{x}-\mathrm{y}$ position in $\mathrm{cm} \ldots \ldots \ldots \ldots \ldots$

4.1. Geometric $\chi^{2}$ of the good three track events of a small sample of the data. This includes $\Xi^{-}$events, $\Omega^{-}$events and all of the backgrounds. .

4.2. Kinematic $\chi^{2}$ of the good three track events of a small sample of the data. This includes $\Xi^{-}$events, $\Omega^{-}$events and their backgrounds. . . . .

4.3. Proton- $\pi^{-}$invariant mass for the good three track events of a small sample of the data. This also includes both $\Xi^{-}$events, $\Omega^{-}$events and

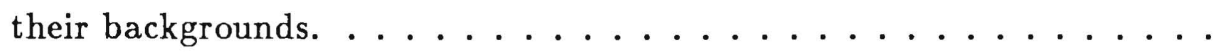

4.4. $\Lambda-\pi^{-}$invariant mass from "good" 3 -track events. This small sample of the data comes from 1 run. . . . . . . . . . . . . 45

4.5. $\Lambda-\mathrm{K}^{-}$invariant mass from "good" 3 -track events. This small sample of the data comes from 1 run. No cuts have been applied to separate $\Omega^{-} \mathrm{s}$ from $\Xi^{-}$s. . . . . . . . . . . . . . . . . 45

4.6. $\Omega^{-}$Monte Carlo vs Data. Shown are the $\Omega^{-}$position at the exit of the charged particle collimator, the radial distance-squared from the target center, and the $\Omega^{-}$momentum. Solid lines represent data. Dashed lines represent the Monte Carlo. (-450 Amp $0 \mathrm{mR}$ Phase 2)........ 
4.7. $\Omega^{-}$Monte Carlo vs Data. The $\Omega^{-}$transverse momentum components at the target and at the collimator exit. Solid lines represent data. Dashed lines represent the Monte Carlo. (-450 Amp $0 \mathrm{mR}$ Phase 2). . . . . . .

4.8. $\Omega^{-}$Monte Carlo vs Data. The momentum of the decay products. Solid lines represent data. Dashed lines represent the Monte Carlo. (-450 Amp $0 \mathrm{mR}$ Phase 2$) \ldots \ldots \ldots \ldots \ldots \ldots \ldots$

4.9. $\Omega^{-}$Monte Carlo vs Data. The $\Lambda$-K and $\mathrm{p}-\pi$ invariant masses and decay vertices. Solid lines represent data. Dashed lines represent the Monte Carlo. (-450 Amp $0 \mathrm{mR}$ Phase 2). . . . . . . . . . . . . . 51

4.10. $\Omega^{-}$Monte Carlo vs Data. The geometric $\chi^{2}$ and the daughter distributions in MWPC C8. The trigger bounday is close to $x=0$. Solid lines represent data. Dashed lines represent the Monte Carlo. (-450 Amp 0 $\mathrm{mR}$ Phase 2$) \ldots \ldots \ldots \ldots \ldots \ldots \ldots \ldots$

4.11. $\Omega^{-}$Monte Carlo vs Data. The daughter distributions in MWPC C9. The trigger boundary is close to $x=0 \mathrm{~cm}$. Solid lines represent data. Dashed lines represent the Monte Carlo. (-450 Amp $0 \mathrm{mR}$ Phase 2). . .

4.12. $\Omega^{-}$Monte Carlo vs Data. Shown are angular distributions of the $\Lambda$ in the $\Omega^{-}$rest frame. Solid lines represent data. Dashed lines represent the Monte Carlo. (-450 Amp $0 \mathrm{mR}$ Phase 2). . . . . . . . . . . . 54

4.13. $\Omega^{-}$Monte Carlo vs Data. Shown are the angular distributions of the proton in the $\Lambda$ rest frame. Solid lines represent data. Dashed lines represent the Monte Carlo. (-450 Amp $0 \mathrm{mR}$ Phase 2). . . . . . . . 55

4.14. $R^{2}$ for events which satisfied all preceeding cuts. The cut was applied at

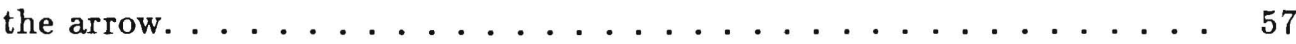

4.15. $\cos \theta_{K^{-}}$versus $\phi_{K^{-}}$for all events preceeding the outlined cut. The cutout band at -0.8 was a result of the $\Xi^{-}$mass cut. . . . . . . . 59

4.16. $\cos \theta_{\mathrm{K}^{-}}$versus $\phi_{\mathrm{K}^{-}}$for Monte Carlo $\Xi^{-}$events. The excess of events centered around $\phi_{\mathrm{K}^{-}}=0$ is from $\Xi^{-}$decays which occurred in the collimator. 60 
4.17. $\Omega^{-}$vertex distribution of the full sample. . . . . . . .

4.18. $\Lambda$ vertex distribution for all $\Omega^{-}$s. The reconstruction program is responsible for the peaks in the distribution at $\mathrm{C} 2$ and C3. It tends to move vertices just upstream of the chamber to the $z$ location of the chamber.

The effect is reproduced in the Monte Carlo events. . . . . . . . 64

4.19. $\pi^{-}$momentum distribution for all $\Omega^{-}$s. . . . . . . . . . . 65

4.20. $\mathrm{K}^{-}$momentum distribution for all $\Omega^{-} \mathrm{s} . \ldots \ldots \ldots \ldots$

4.21. Geometric $\chi^{2}$ for all $\Omega^{-}$events. . . . . . . . . . . . . 66

4.22. Proton momentum distribution from all $\Omega^{-}$events. . . . . . . . . . . 66

4.23. $\Omega^{-}$momentum distribution from all $\Omega^{-}$events. $\ldots \ldots \ldots$. . . . . 67

4.24. $\mathrm{p}-\pi^{-}$invariant mass distribution of all $\Omega^{-}$events. . . . . . . . . 68

4.25. Lambda - $\mathrm{K}^{-}$invariant mass distribution for all $\Omega^{-} \mathrm{s}$. These are two plots of the same data. The bottom plot has a log vertical scale. The position of the mass cut is shown by the arrows. . . . . . . . . 69

4.26. Lambda $-\pi^{-}$invariant mass distribution. . . . . . . . . . . . 76

4.27. Geometric $\chi^{2}$ for $\Xi^{-}$events. . . . . . . . . . . . . . 77

4.28. $\Xi^{-}$momentum distribution. $\ldots \ldots \ldots \ldots \ldots$

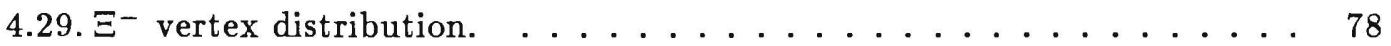

4.30. $\Lambda$ vertex distribution. . . . . . . . . . . . . 79

5.1. Relative orientation of coordinate systems $S$ and $S^{\prime} \ldots \ldots \ldots$. . . 84

5.2. Proton angles in the $\Lambda$ rest frame. . . . . . . . . . 86

5.3. Phase $1 \Omega^{-}$Polarization components. Plotted are $P_{x}$ vs. $P_{z}$ for the 5 fields. These are from Table 5.2. $\gamma_{\Omega}$ is assumed +1 for this plot. . . . 96

5.4. Phase 1 Polarization. These are the results of the grand fit assuming the $\mathrm{g}$-factor is 2.40 as measured in Phase 2. The results are presented assuming $\gamma=+1$. If $\gamma_{\Omega}$ is -1 , scale the polarization and uncertainties by

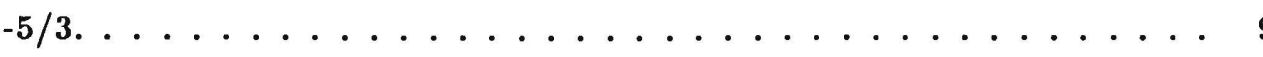


5.5. Phase $1 \hat{x}$ Bias. These are the results of the grand fit assuming the g-factor is 2.40 as measured in Phase $2 \ldots \ldots . \ldots 99$

5.6. Phase $1 \hat{z}$ Bias. These are the results of the grand fit assuming the g-factor is 2.40 as measured in Phase 2. . . . . . . . . . 100

5.7. Phase $1 \Xi^{-}$Polarization components. Plotted are $P_{x}$ vs. $P_{z}$ for each of the 5 field integrals. . . . . . . . . . . . . . . . 104

5.8. $\Omega^{-}$asymmetry components from Phase 2 . The 3 lower momentum points are the -450 Amp sample. The highest momentum point is the $-1000 \mathrm{amp}$ sample. The targeting angle is indicated with a letter symbol. . . . . 108

5.9. Phase $2 \Omega^{-}$Polarization components. Plotted are $P_{x}$ vs. $P_{z}$ for the 4 data points in Table 5.13. The results are presented assuming $\gamma=+1$. If $\gamma_{\Omega}$ is -1 , scale the polarization and uncertainties by $-5 / 3 . \ldots 110$

5.10. Phase 2. $\Omega^{-}$polarization at the production target as a function of momentum. Again, $\gamma_{\Omega}$ is assumed to be +1 . For comparison, the $\Xi^{-}$ polarization from the next section is included. . . . . . . . . . . . 114

5.11. $\hat{x}$ Asymmetry of Phase $2 \Xi^{-}$s. This is the main component of $\alpha_{\Lambda} P_{\Lambda}$. The asymmetry is shown as a function of $\Xi^{-}$momentum for the 3 Hyperon Magnet currents and 3 targeting angles. . . . . . . . . . . 116

5.12. $\hat{y}$ Asymmetry of Phase $2 \Xi^{-}$s. This is the parity-violating component of $\alpha_{\Lambda} P_{\Lambda}$. The asymmetry is shown as a function of $\Xi^{-}$momentum for the 3 Hyperon Magnet currents and 3 targeting angles. . . . . . . . . . 117

5.13. $\hat{z}$ Asymmetry of Phase $2 \Xi^{-}$s. The $\hat{x}$ component of $\alpha_{\Lambda} P_{\Lambda}$ precesses onto this axis in the Hyperon Magnet. The asymmetry is shown as a function of momentum for the the 3 Hyperon Magnet currents and 3 targeting

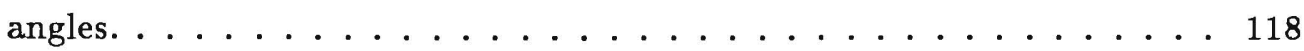

5.14. Phase $2 \Xi^{-}$Polarization components. Plotted are $P_{x}$ vs. $P_{z}$ for the 9 data points of Table 5.19. . . . . . . . . . . . . . 120 
5.15. Monte Carlo Bias vs. Data Bias. The points are shown as a function of momentum for $B_{x}, B_{y}$, and $B_{z} \ldots \ldots \ldots \ldots \ldots$

6.1. Phase $2 \Xi^{-}$precession angles vs. field integral. The precession angle is provided in radians and the field integral in tesla-meters. The fit was

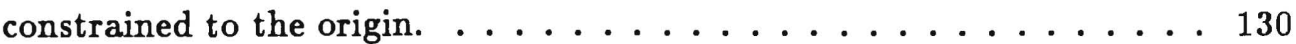

6.2. Phase $2 \Omega^{-}$precession angle vs. field integral. This fit was constrained

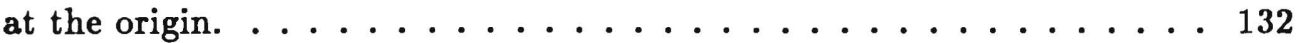

6.3. Lines of constant $\sigma$. The horizontal axis is the polarization. The vertical axis is the magnetic moment. $\gamma$ is assumed to be +1 in this plot. . . 134

B.1. Neutron production by $400 \mathrm{GeV} / \mathrm{c}$ protons. The targeting angles are 0.7 , 1.6, 3.0, 5.0, 6.0, 8.0 and $10.0 \mathrm{mR}$. The points are the data. The lines are the fit at 3.0 and $5.0 \mathrm{mR}$ and the interpolation at $4.0 \mathrm{mR}$. . . .

B.2. $\Lambda$ production by $400 \mathrm{GeV} / \mathrm{c}$ protons. The targeting angles are $0.0,2.0$, 3.5, 7.3, and $9.8 \mathrm{mR}$. The target was $\mathrm{Cu}$. The lines are the result of the

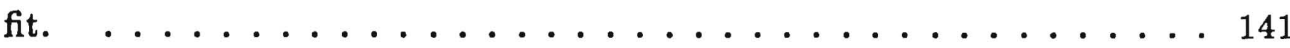

B.3. $\Xi^{0}$ production by $400 \mathrm{GeV} / \mathrm{c}$ protons. The targeting angles are $0.0,2.0$, 3.5, 7.3, and $9.8 \mathrm{mR}$. The target was $\mathrm{Cu}$. The lines are the result of the

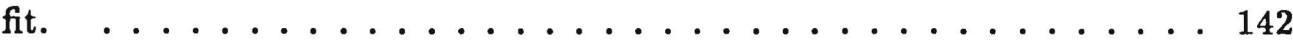

B.4. Relative $\Omega^{-}$and $\Xi^{-}$rates from production by a decaying neutral beam of widespread momentum. . . . . . . . . . . . . . 146

B.5. Predictions for Phase $2 \Omega^{-}$and $\Xi^{-}$polarization. . . . . . . . 150

B.6. Modified predictions for Phase $2 \Omega^{-}$and $\Xi^{-}$polarization. . . . . 151 


\section{Chapter 1}

\section{Introduction}

A group of physicists from Rutgers, The State University of New Jersey; The University of Minnesota; The University of Michigan; and Fermi National Accelerator Laboratory (FNAL) performed FNAL experiment E756 from June, 1987 to February, 1988. The main purpose of the experiment was to measure to the magnetic dipole moment of the $\Omega^{-}$hyperon. The experimental method, called the spin-precession technique, was to produce a polarized beam of $\Omega^{-} \mathrm{s}$ and measure the precession of the polarization vector in a known magnetic field. Two different methods were used in the attempt to produce the polarized $\Omega^{-}$s. The first method was to produce them with unpolarized protons in the inclusive reaction

$$
p\left(800 G e \mathrm{I}^{-}\right)+B e \Rightarrow \Omega^{-}+X
$$

where $X$ is not observed. Preliminary analysis of the data suggested that the $\Omega^{-}$polarization was not significantly different from zero. Another method was then employed with two stage production of hyperons. This method was

$$
\begin{gathered}
p(800 \mathrm{GeV})+C u \Rightarrow N \uparrow \\
N \uparrow+C u \Rightarrow \Omega^{-}+X
\end{gathered}
$$

where $N \uparrow$ stands for a beam containing unobserved polarized neutral particles. These two methods of producing $\Omega^{-}$s are referred to as Phase 1 and Phase 2 respectively. The Phase 2 method produced $\Omega^{-} \mathrm{s}$ with a polarization of $-0.069 \pm 0.022$. The $\Omega^{-}$magnetic moment was determined to be $-2.02 \pm 0.16 \pm 0.12$ nuclear magnetons.

A sample of 13.6 million $\Xi^{-}$events was collected during Phase 1 and Phase 2. A fraction of these events enabled us to perform a precision study of the $\Xi^{-}$polarization and 
magnetic moment. The average polarization of the Phase $1 \Xi^{-}$sample was $-0.109 \pm 0.005$. The average polarization of $\Xi^{-}$s produced in Phase 2 was $-0.112 \pm 0.005$. The magnetic moment was determined to be $-0.650 \pm 0.005 \pm 0.002$ nuclear magnetons.

This thesis reports on the polarization and magnetic moment results from the

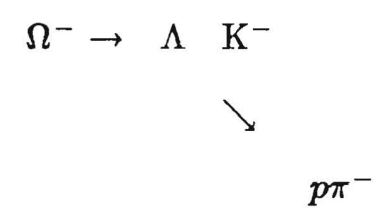

decay chain in Phase 1 and Phase 2. The Phase 1 and Phase 2 polarization and magnetic moment results of the

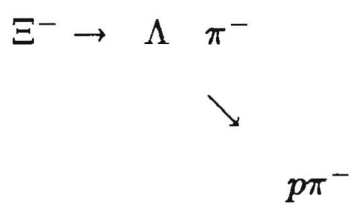

decay chain are also presented. Chapter 2 describes the experimental and theoretical motivation. Chapter 3 describes the apparatus. Chapter 4 describes the $\Omega^{-}$and $\Xi^{-}$ reconstruction and event selection. Chapter 5 contains a discussion of the polarization analysis and results of the Phase 1 and Phase $2 \Omega^{-}$and $\Xi^{-}$samples. Chapter 6 describes contains the $\Omega^{-}$and $\Xi^{-}$magnetic moment analysis. Chapter 7 is a summary of the results. Appendix A describes spin-precession in a magnetic field. Appendix B contains a discussion the contents of the neutral beam and predictions for $\Xi^{-}$and $\Omega^{-}$polarization from spin transfer. Appendix C contains a description of the design of new, large 1mm pitch MWPCs built for E756. 


\section{Chapter 2}

\section{Motivation}

This chapter provides background information useful in understanding the purpose of measuring the polarization and magnetic moments of hyperons. First, a summary of current hyperon polarization results is presented. Then properties of the $\Omega^{-}$are reviewed. The third section is a transition to the discussion of magnetic moment calculations and polarization theories presented in Sections 4 and 5.

\subsection{Experimental Background}

Experiments performed during the last 13 years by this collaboration discovered that polarization was a general feature of high-energy hyperon production. This phenomena, a priori unexpected, was originally discovered by FNAL E8 which studied inclusive $\Lambda$ production by $300 \mathrm{GeV}$ protons [1]. In a flurry of experiments from 1976 to 1981, inclusive hyperon polarization was studied as a function of beam energy, kinematic variables and target materials [2] [3]. The general features of the polarization were that for the reaction proton + target $\rightarrow$ hyperon $+X$; the polarization lie in the direction

$$
\hat{n}=\frac{\vec{k}_{\text {proton }} \times \vec{k}_{\text {hyperon }}}{\left|\vec{k}_{\text {proton }} \times \vec{k}_{\text {hyperon }}\right|}
$$

at the target, where $\vec{k}$ is the particle momentum. The polarization was found to increase with transverse momentum, $P_{t}$, for $0<P_{t}<\sim 1.5 \mathrm{GeV} / \mathrm{c}$, and plateau for higher $P_{t}$. In other words, it was necessary to observe hyperons produced at an angle with respect to the direction of the incident beam. The polarization increased with $x_{f}$, the fraction of the incident beam carried by the hyperon; was not strongly dependent on the energy of the incident beam from 20 to $400 \mathrm{GeV} / \mathrm{c}$; and was slightly lower for targets with high 


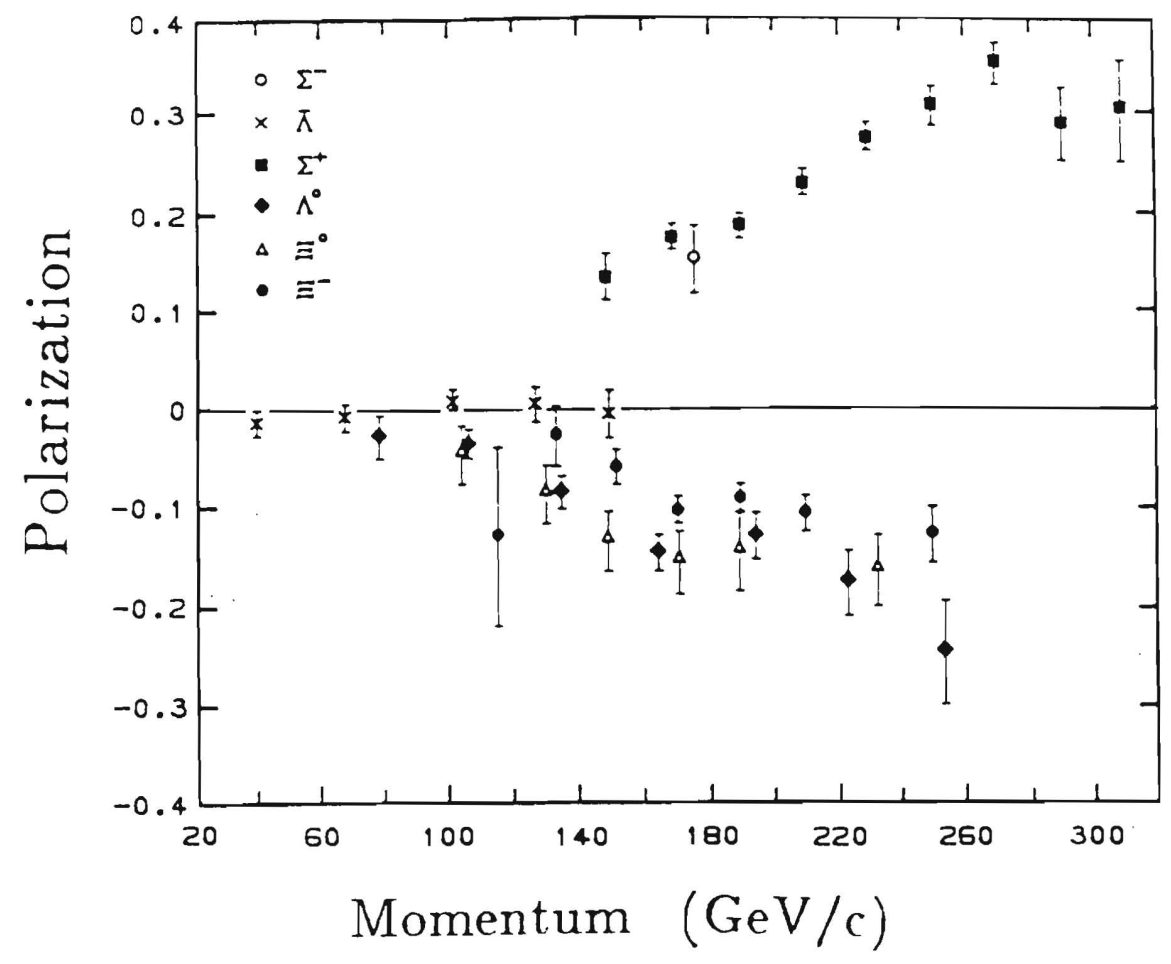

Figure 2.1: Hyperon polarization from $400 \mathrm{GeV} / \mathrm{c}$ protons. The production angle was $5 \mathrm{mR}$ except for the $\bar{\Lambda}$ and $\Sigma^{-}$where the production angle was $7.5 \mathrm{mR}$. The target was Be.

atomic number. Polarizations as high as $\vec{P}_{\Lambda}=-.34 \hat{n}$ were observed. For the other hyperons, $P_{\Sigma^{+}} \approx P_{\Sigma^{-}} \approx-P_{\Lambda}$ and $P_{\Xi^{-}} \leq P_{\Xi^{0}} \approx P_{\Lambda^{\prime}}$. On the other hand, $P_{\text {proton }}=$ $P_{\bar{\Lambda}}=0$. Reference $[2]$ presents a review of many of these results. The $\Omega^{-}$polarization data was inconclusive due to low statistics [4]. These trends are summarized in Figure 2.1 which shows hyperon polarization as a function of kinematic variables for production by $400 \mathrm{GeV} / \mathrm{c}$ protons [5]. Meanwhile, other collaborations discovered that high-energy $\mathrm{K}^{-} p$ reactions produced polarized $\Lambda \mathrm{s}, \bar{p} p$ reactions produce polarized $\bar{\Lambda} \mathrm{s}, \mathrm{K}^{+} p$ reactions produced polarized $\bar{\Lambda}$ s (all from Reference [6]), and possibly, $\pi^{-} p$ reactions produced polarized $\Lambda \mathrm{s}$ [7]. A paper exists reporting that neutrons produced polarized $\Lambda_{c}^{+} s[8]$.

The hyperons decay via the parity-violating weak interaction. In the decay $B \rightarrow B^{\prime}+$ meson, the $B^{\prime}$ are emitted in a preferred direction. Conservation of angular momentum 


\begin{tabular}{||c|c||}
\hline Baryon & Magnetic Moment (Nuclear Magnetons) \\
\hline$p$ & 2.7928 \\
\hline $\bar{p}$ & $-2.795 \pm 0.019$ \\
\hline$n$ & -1.9130 \\
\hline$\Lambda$ & $-0.613 \pm 0.004$ \\
\hline$\left|\left(\Sigma^{0}-\Lambda\right)\right|$ & $1.61 \pm 0.08$ \\
\hline$\Sigma^{+}$ & $2.42 \pm 0.05$ \\
\hline$\Sigma^{-}$ & $-1.157 \pm 0.025$ \\
\hline$\Xi^{0}$ & $-1.250 \pm 0.014$ \\
\hline$\Xi^{-}$ & $-0.679 \pm 0.031$ \\
\hline$\Omega^{-}$ & \\
\hline
\end{tabular}

Table 2.1: Magnetic moment measurements. Before E756. The nuclear magneton is defined by $e \hbar / 2 m_{p} c$.

requires that the $B^{\prime}$ have a net helicity alignment. This means that the decay products of a polarized beam of hyperons had an asymmetry proportional to the polarization. Measuring this asymmetry provided a means of measuring the polarization. In all of these experiments, a large magnet located downstream of the production target was used to select the charge and, for the charged particles, the momentum of the desired particles. This magnet also caused the particle's polarization vectors to precess through an angle proportional to their magnetic moment. Taking advantage of this precession, predecessors of E756 measured the magnetic moments of the $\Lambda, \Xi^{0}, \Xi^{-}, \Sigma^{-}$, and $\Sigma^{+}$. This technique of measuring magnetic moments is known as the spin-precession method. Table 2.1 contains the measurements of the known hadron magnetic moments including the $\Sigma-\Lambda$ transition magnetic moment which was measured by means of the Primakov Effect and the latest high precision measurement of the $\Sigma^{-}$magnetic moment obtained by measuring the fine-structure line-broadening in exotic atom states. All of these measurements are reported in the Particle Data Book [9] except for a recent spinprecession measurement of the $\Xi^{-}$magnetic moment [10]. The $\Xi^{-}$magnetic moment reported in Table 2.1 is the weighted average of References [10] and [11]. As for the $\Omega^{-}$, Luk et al. [4] concluded $1743 \Omega^{-}$collected in E620 were not significantly polarized and made no measurement of the magnetic moment. 


\subsection{Omega Minus Properties}

The following summarizes the current knowledge about the $\Omega^{-}$. It's discovery in bubble chamber interactions at Brookhaven in 1964[12] was an initial triumph of the naive quark model which predicted it's existance as the three-strange-quark member of the spin-3/2 baryon decuplet. The $\Omega^{-}$mass is $1672.43 \pm 0.32 \mathrm{GeV} / \mathrm{c}^{2}$. The lifetime is $0.822 \pm$ $0.012 \times 10^{-10}$ seconds. The principal decay modes and corresponding branching ratios are $\Omega^{-} \rightarrow \Lambda \mathrm{K}^{-}(64.8 \pm 0.8 \%), \Omega^{-} \rightarrow \Xi^{0} \pi^{-}(23.6 \pm 0.7 \%)$, and $\Omega^{-} \rightarrow \Xi^{-} \pi^{0}(8.6 \pm 0.04 \%)$. The spin and parity, while assumed to be $\frac{3}{2}^{+}$, have never been measured. As the $\Omega^{-}$ decays via the weak interaction, the decay is not required to conserve parity. However, it must conserve angular momentum. If the $\Omega^{-}$has spin $\mathrm{J}$, and decays to a spin-1/2 baryon and a spin- 0 meson, the decay products must have orbital angular momentum $\mathrm{J} \pm 1 / 2$. The final state parity is then $(-1)(-1)^{J \pm 1 / 2}$. So, if the $\Omega^{-}$has spin-parity $\frac{3}{2}^{+}$, the $P$-wave $(L=1)$ is parity- conserving and the $D$-wave $(L=2)$ is parity-violating. Writing the amplitudes for decay by $\mathrm{P}$ and $\mathrm{D}$-waves as $A_{J \pm 1 / 2}$, the asymmetry parameters are described by

$$
\begin{gathered}
\alpha=2 \frac{\operatorname{Re}\left(A_{J-1 / 2}^{*} A_{J+1 / 2}\right)}{\left|A_{J-1 / 2}\right|^{2}+\left|A_{J+1 / 2}\right|^{2}} \\
\beta=2 \frac{I m\left(A_{J-1 / 2}^{*} A_{J+1 / 2}\right)}{\left|A_{J-1 / 2}\right|^{2}+\left|A_{J+1 / 2}\right|^{2}} \\
\gamma=\frac{\left|A_{J-1 / 2}\right|^{2}-\left|A_{J+1 / 2}\right|^{2}}{\left|A_{J-1 / 2}\right|^{2}+\left|A_{J+1 / 2}\right|^{2}},
\end{gathered}
$$

with the constraint that $\alpha^{2}+\beta^{2}+\gamma^{2}=1$. The known asymmetry parameters of the $\Omega^{-} \rightarrow \Lambda K^{-}, \Omega^{-} \rightarrow \Xi^{0} \pi^{-}$and $\Omega^{-} \rightarrow \Xi^{-} \pi^{0}$ decay modes are $\alpha_{\Lambda K^{-}}=-0.026 \pm 0.026$, $\alpha_{\Xi^{0} \pi^{-}}=0.09 \pm 0.14$ and $\alpha_{\Xi^{-} \pi^{0}}=0.05 \pm 0.21$. $\beta$ and $\gamma$ have not been measured for any of these decay modes. $\beta$ is expected to be zero up to tiny contributions from possible final-state interactions or CP violation. $\gamma$ is expected to be +1 from theoretical predictions [13] - [15]. Thus, the parity-conserving amplitudes are thought to dominate the decay modes. This summary of $\Omega^{-}$results are the products of many experiments and can be found in the Particle Data Book [9]. 


\subsection{Theoretical Background}

The picture of the hadron structure has evolved from the simple quark model of the early-1960's which envisioned hadrons as consisting solely of non-interacting localized quarks. The dilemma posed by anti-symmetrization of the wavefunction of the spin- $3 / 2$ baryons was resolved by introduction of a new quantum number called color. Because of the results of lepton-nucleon scattering experiments performed in the late 1960's, the quark-parton model of hadrons emerged where quarks are tightly confined in hadrons but interact only weakly at short distances (i.e. high transverse momenta). This shortdistance property of strong interactions is called asymptotic freedom.

The discovery that asymptotic freedom could occur in non-abelian gauge theories made Quantum Chromodynamics (QCD) the leading theory of the strong interaction. QCD is a gauge theory with 8 massless spin-1 bosons (gluons) which carry color, the strong force charge. Unlike photons, the carriers of the electromagnetic force between electrically charged objects, gluons not only interact with the colored quarks but among themselves as well.

Hadrons, in QCD, are color-neutral bound states of quarks and gluons. However, the remarkable property that QCD is asymptotically free makes "static" properties of hadrons such as their charge radius, mass spectrum and magnetic moment and longdistance effects such as inclusive polarization and production cross-sections impossible to calculate at present with perturbative QCD techniques. The non-perturbative field theoretical approach of lattice gauge theory has been used to recreate hadron masses and shows promise towards eventually modeling hadron structure sucessfully but suffers because of the large amounts of computer time required to make a precise calculation. Phenomenological models of hadrons based on QCD have prospered including the naive quark model and various bag models. Corrections have been added to these which allow the models to fit many experimental parameters with varying degrees of success. 


\subsection{Magnetic Moment Calculations}

One static property determined from particle's structure is its magnetic moment. The magnetic moment describes the particles behavior in a magnetic field. A particle with angular momentum, $\vec{J}$, experiences a torque

$$
\frac{\overrightarrow{d J}}{d t}=\vec{\mu} \times \vec{B}
$$

and an interaction energy

$$
\delta E=-\vec{\mu} \cdot \vec{B}
$$

in a magnetic field, $\vec{B}$. The magnetic moment gives clues about the particle's structure. For example, the magnetic moment of a spin-S pointlike particle is

$$
\vec{\mu}=\frac{e g_{s}}{2 m c} \vec{S}
$$

where $e$ is the particle's charge, $m$ its mass, and $S$ its intrinsic spin angular momentum. The value of $g_{s}$ is 2 . But for an extended object such as a baryon, the magnetic moment is

$$
\mu_{b}=\left\langle\Psi_{b} \uparrow\left|\sum_{i} \frac{e_{i}}{2 m_{i} \hbar c(J / \hbar+1)}\left(g_{s} \vec{S} \cdot \vec{J}+g_{l} \vec{L} \cdot \vec{J}\right)_{i}\right| \Psi_{b} \uparrow\right\rangle,
$$

where $e_{i}, m_{i}, L, S, J$ are the constituent's charge, mass, orbital angular momentum, intrinsic spin, and total angular momentum. That is, the magnetic moment is the sum of the Dirac moment and the contribution from the orbital angular momenta of the charged particles which make up the baryon. The value of $g_{l}$ is 1 . The fact that the neutron has a non-zero magnetic moment was one of the original clues that the baryons are composed of smaller particles.

The naive quark model assumes that the hadron constituents (only quarks) have $L=0$. Under this assumption, the baryon magnetic moments are given by

$$
\mu_{b}=\left\langle\Psi_{b} \uparrow\left|\sum_{i} \mu_{z_{b}}\right| \Psi_{b} \uparrow\right\rangle
$$

The wavefunctions for the spin- $1 / 2$ octet baryons and some of the spin- $3 / 2$ decuplet baryons, including the $\Omega^{-}$are given in Table 2.2. The predictions for those particles 


\begin{tabular}{cc} 
Baryon & Wavefunction \\
\hline \hline $\mathrm{p} \uparrow$ & $\sqrt{2 / 3} \mathrm{u} \uparrow \mathrm{u} \uparrow \mathrm{d} \downarrow-\sqrt{1 / 6}(\mathrm{u} \uparrow \mathrm{u} \downarrow+\mathrm{u} \downarrow \mathrm{u} \uparrow) \mathrm{d} \downarrow$ \\
$\mathrm{n} \uparrow$ & $\sqrt{2 / 3} \mathrm{~d} \uparrow \mathrm{d} \uparrow \mathrm{u} \downarrow-\sqrt{1 / 6}(\mathrm{~d} \uparrow \mathrm{d} \downarrow+\mathrm{d} \downarrow \mathrm{d} \uparrow) \mathrm{u} \downarrow$ \\
$\Lambda \uparrow$ & $\sqrt{1 / 2}(\mathrm{u} \uparrow \mathrm{d} \downarrow-\mathrm{u} \downarrow \mathrm{d} \uparrow) \mathrm{s} \uparrow$ \\
$\Sigma^{+} \uparrow$ & $\sqrt{2 / 3} \mathrm{u} \uparrow \mathrm{u} \uparrow \mathrm{s} \downarrow-\sqrt{1 / 6}(\mathrm{u} \uparrow \mathrm{u} \downarrow+\mathrm{u} \downarrow \mathrm{u} \uparrow) \mathrm{s} \uparrow$ \\
$\Sigma^{0} \uparrow$ & $\sqrt{2 / 3} \mathrm{u} \uparrow \mathrm{d} \uparrow \mathrm{s} \downarrow-\sqrt{1 / 6}(\mathrm{u} \uparrow \mathrm{d} \downarrow+\mathrm{u} \downarrow \mathrm{d} \uparrow) \mathrm{s} \uparrow$ \\
$\Sigma^{-} \uparrow$ & $\sqrt{2 / 3} \mathrm{~d} \uparrow \mathrm{d} \uparrow \mathrm{s} \downarrow-\sqrt{1 / 6}(\mathrm{~d} \uparrow \mathrm{d} \downarrow+\mathrm{d} \downarrow \mathrm{d} \uparrow) \mathrm{s} \uparrow$ \\
$\Xi^{0} \uparrow$ & $\sqrt{2 / 3} \mathrm{~s} \uparrow \mathrm{s} \uparrow \mathrm{u} \downarrow-\sqrt{1 / 6}(\mathrm{~s} \uparrow \mathrm{s} \downarrow+\mathrm{s} \downarrow \mathrm{s} \uparrow) \mathrm{u} \uparrow$ \\
$\Xi^{-} \uparrow$ & $\sqrt{2 / 3} \mathrm{~s} \uparrow \mathrm{s} \uparrow \mathrm{d} \downarrow-\sqrt{1 / 6}(\mathrm{~s} \uparrow \mathrm{s} \downarrow+\mathrm{s} \downarrow \mathrm{s} \uparrow) \mathrm{d} \uparrow$ \\
$\Omega^{-} \uparrow$ & $\mathrm{s} \uparrow \mathrm{s} \uparrow \mathrm{s} \uparrow$ \\
$\Delta^{++} \uparrow$ & $\mathrm{u} \uparrow \mathrm{u} \uparrow \mathrm{u} \uparrow$ \\
$\Delta^{-} \uparrow$ & $\mathrm{d} \uparrow \mathrm{d} \uparrow \mathrm{d} \uparrow$ \\
\hline
\end{tabular}

Table 2.2: Naive quark model wavefunctions. These are simplified wavefunctions presented for the purpose of illustrating the baryon's flavor and spin content. To obtain the complete wavefunctions, it is necessary to permute the quark flavors and multiply by the anti-symmetric color singlet.

magnetic moments as well as the $\Lambda-\Sigma^{0}$ transition magnetic moment are given in Table 2.3. Corrections to these values have been suggested which incorporate the effects of

1. changing the quark wavefunctions to include admixtures of quarks with orbital angular momentum [16] - [19],

2. adding anomalous magnetic moments to the dirac moments of the quarks [20],

3. $\Lambda-\Sigma^{0}$ mixing $[21]$,

4. using an arbitrary (odd) number of colors while retaining the overall wavefunction antisymmetry $[22]$, and

5. supposing that the contribution of the individual quark magnetic moments depends on the hyperon in which they sit [23] - [25].

Corrections 1, 3, and 4 involve modifying the quark wavefunctions. Corrections 2 and 5 involve modifying the contribution from each quark. 


\begin{tabular}{||c|c|c||}
\hline Baryon & Magnetic Moment Prediction & Nuclear Magnetons \\
\hline $\mathrm{p}$ & $4 / 3 \mu_{u}-1 / 3 \mu_{d}$ & \multirow{2}{*}{ Input } \\
\cline { 1 - 2 } $\mathrm{n}$ & $4 / 3 \mu_{d}-1 / 3 \mu_{u}$ & \multirow{2}{*}{} \\
\hline$\Lambda$ & $\mu_{s}$ & -1.630 \\
\hline$\Sigma^{0}-\Lambda$ & $\sqrt{1 / 3}\left(\mu_{d^{-}} \mu_{u}\right)$ & $2.673 \pm 0.001$ \\
\hline$\Sigma^{+}$ & $4 / 3 \mu_{u}-1 / 3 \mu_{s}$ & $0.791 \pm .001$ \\
\hline$\Sigma^{0}$ & $2 / 3\left(\mu_{u}+\mu_{d}\right)-1 / 3 \mu_{s}$ & $-1.091 \pm 0.001$ \\
\hline$\Sigma^{-}$ & $4 / 3 \mu_{d}-1 / 3 \mu_{s}$ & $-1.435 \pm 0.005$ \\
\hline$\Xi^{0}$ & $4 / 3 \mu_{s}-1 / 3 \mu_{u}$ & $-0.493 \pm 0.005$ \\
\hline$\Xi^{-}$ & $4 / 3 \mu_{s}-1 / 3 \mu_{d}$ & $-1.839 \pm 0.012$ \\
\hline$\Omega^{-}$ & $3 \mu_{s}$ & 5.555 \\
\hline$\Delta^{++}$ & $3 \mu_{u}$ & -2.916 \\
\hline$\Delta^{-}$ & $3 \mu_{d}$ & \\
\hline
\end{tabular}

Table 2.3: Naive quark model (without corrections) magnetic moment predictions. The numerical values come from using the $\mathrm{p}, \mathrm{n}$, and $\Lambda$ wavefunctions to determine the up, down and strange quark magnetic moments. Here, $\mu_{u}=1.8516, \mu_{d}=-0.97184$ and $\mu_{s}=-0.613 \pm 0.004$ nuclear magnetons.

Bag models are another QCD-inspired phenomonological attempt at modeling the structure of hadrons. Hadrons are envisioned as bubbles of quark gas immersed in the vacuum. In the simplest bag model, the quarks are massless, non-interacting, free particles confined to a domain with a spherical boundary. The pressure exerted by the quarks on the bag boundary is compensated by the pressure exerted by the vacuum and the surface tension of the domain walls. Thus, the hadron mass can be written

$$
M=\frac{a_{1} n}{r}+a_{2} 4 \pi r^{2}+a_{3} \frac{4}{3} \pi r^{3}
$$

where $\mathrm{n}$ is the number of quarks, $r$ is the bag radius to be determined by minimizing the mass, and $a_{1}, a_{2}$, and $a_{3}$ are free parameters to be determined from the data. The magnetic moment of a bag model hadron is calculated from the energy shift due to a weak, constant external magnetic field. More explicitly, for a magnetic field $\vec{B}$, the magnetic vector potential $\vec{A}$ can be taken as $1 / 2 \vec{B} \times \vec{r}$ and the electromagnetic interaction energy as

$$
-\vec{\mu} \cdot \vec{B}=-\int \vec{j} \cdot \vec{A} d^{3} r
$$


where the electromagnetic current operator is

$$
j_{\nu}=i \sum_{q} e_{q} \Psi_{q}^{\dagger} \gamma_{4} \gamma_{\nu} \Psi_{q}
$$

The wavefunctions are the Bessel function solutions to the Dirac Equation for free quarks in a spherical bag. Thus, taking $\vec{B}=B_{z} \hat{z}$,

$$
\mu_{b}=\frac{1}{2} \sum_{q} e_{q} \int \Psi_{q}^{\dagger} \uparrow\left(i \gamma_{4} \gamma_{k} \times \vec{r}\right)_{z} \Psi_{q} \uparrow d r^{3}
$$

The first bag model calculations provided marginally satisfactory results for the neutron, proton [26], and hyperon magnetic moments [27]. Corrections to the bag model calculations include the effects of

1. adding gluons to the bag $[28]-[29]$,

2. penetration of the bag boundary by virtual $q \bar{q}$ pairs [30],

3. giving the quarks mass $[31]$,

4. using bag potentials other than a spherical cavity [32] - [34], and

5. center-of-mass corrections to the quark wavefunctions [35]- [36].

All of these effects change the charge radius, and thus the magnetic moment of the hadron.

The $\Omega^{-}$has been ignored, for the most part, in reports making magnetic moment predictions. However, theoretical predictions do exist. The predictions for the $\Omega^{-}$ magnetic moment range from -1.3 to -2.52 nuclear magnetons (n.m.). The two naive quark model predictions which include the effects of giving the quarks orbital angular momentum agree on their prediction of $\mu_{\Omega^{-}}$. The $\Omega^{-}$is free of such effects because each quark must have the same wavefunction and the $\Omega^{-}$must remain in the ground state. Thus, none of the quarks has orbital angular momentum. The magnetic moment is three times the strange quark moment, which is no longer $\mu_{\Lambda}$, but a fitted parameter. Table 2.4 contains a compilation of the predictions including a Lattice Gauge Theory prediction by C. Bernard et al. [37]. 


\begin{tabular}{||c|c|c||}
\hline Reference & Model & Nuclear Magnetons \\
\hline$[17]$ & NQM + Orbital Angular Momentum & $\mathbf{- 2 . 2 6 \pm 0 . 0 9}$ \\
\hline$[18]$ & NQM + Orbital Angular Momentum & $\mathbf{- 2 . 2 5 \pm 0 . 0 9}$ \\
\hline$[23]$ & NQM + Quark-Quark Interactions & -1.37 \\
\hline$[24]$ & NQM + Baryon Dependent Corrections & -2.33 \\
\hline$[25]$ & NQM + Baryon Dependent Corrections & -1.3 \\
\hline$[29]$ & Bag + Gluon Interaction Energy & -2.52 \\
\hline$[36]$ & Bag + Pions Penetrate Bag Walls & -1.51 \\
\hline$[37]$ & Lattice Gauge Theory & $-1.7 \pm 0.6$ \\
\hline
\end{tabular}

Table 2.4: Theoretical predictions of the $\Omega^{-}$magnetic moment.

\subsection{Polarization Theories}

The low-energy, long-distance phenomena of polarization in inclusive hyperon production at $P_{t}$ s on the order of $1 \mathrm{GeV} / \mathrm{c}$ is also uncalculable, at present, with perturbative QCD. As a result, three phenomenological models have arisen which attempt to explain the experimental results. All of the models make the assumption that the maximum number of valence quarks are carried from the beam particle to the outgoing hyperon, along with their part of the $\mathrm{SU}(6)$ naive quark wavefunctions. Each model has a different origin for the polarization and each model has specific problems.

In the Lund Model [38], a color dipole field stretches between the collision point and the beam valence quarks to be retained by the hyperon. The field produces a $q \bar{q}$ pair. The sea quark which hadronizes with the hyperon has some transverse momentum with respect to the original beam direction. Thus the sea quark-antiquark pair has some orbital angular momentum which is compensated by it's net spin angular momentum. Because the contribution to $P_{t}$ of the polarized sea quark increases with the $P_{t}$ of the hyperon, so does the hyperon polarization. Figure 2.2 provides a sketch of this mechanism. In this model, the initial spin asymmetry is carried entirely by the sea quarks. For production of particles with more than one sea quark, the di-quark-antiquark pair is polarized in a similar manner.

In the Thomas Precession Model of DeGrand and Miettinen (D-M) [39], a general 


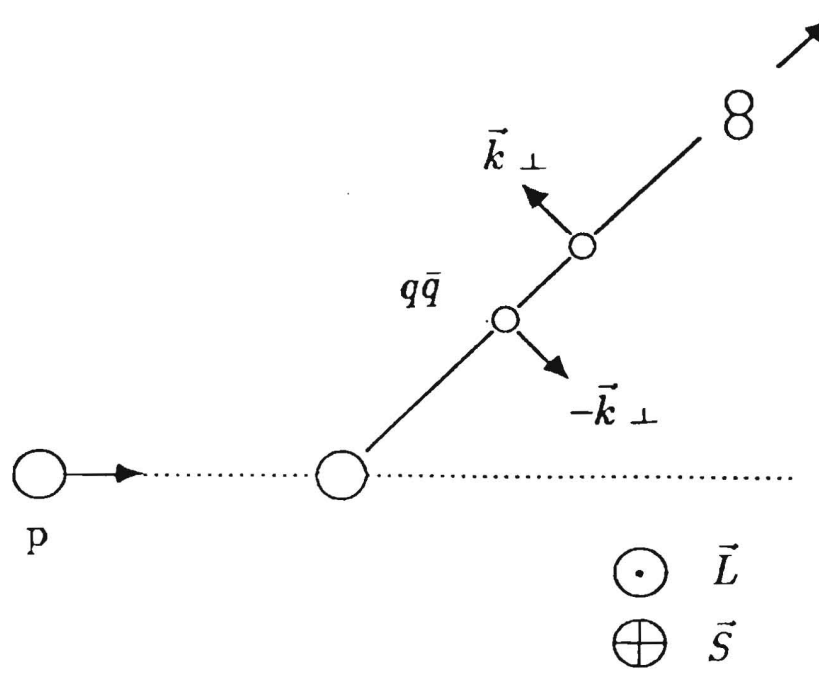

Figure 2.2: Lund model of polarization. A quark or diquark pair is created along a color force string. The transverse momentum of the quark or diquark pair results in an angular momentum which is compensated by its spin.

rule is formulated. The outgoing baryons are formed from sea quarks which, accelerated from the collision point, tend to recombine with their spins down with respect to $\vec{k}_{\text {beam }} \times \vec{k}_{\text {hyperon }}$, and from valence quarks which, decelerated, tend to recombine with their spins up. This occurs because the scattering Hamiltonian contains Thomas Precession terms proportional to the spin of the sea and valence quarks and their respective Thomas precessions while being accelerated (or decelerated) during recombination. In addition to providing the polarization mechanism, the $\mathrm{D}$-M model presents a simple parameterization for polarization observables of any forward-production hadron reaction using the $\mathrm{SU}(6)$ wavefunctions, including production by polarized beam particles.

The QCD-based model of Szwed [40] attempts to calculate polarization resulting from scattering the sea quark off an effective external gluon field produced during the collision. The effective field approximates all of the QCD subprocesses. The calculation is carried out to second order PQCD. The resulting sea quark polarization is

$$
P=\left(2 C \alpha_{s} m \frac{k}{E^{2}}\right) \frac{\sin ^{3}(\theta / 2) \ln \sin (\theta / 2)}{\left[1-(k / E)^{2} \sin ^{2}(\theta / 2)\right] \cos (\theta / 2)},
$$


where $m, k, E$, and $\theta$ are the mass, momentum, energy and scattering angle of the seaquark, $C$ is the strength of the color field, and $\alpha_{s}$ is the strong coupling constant. The beam particle valence quarks (spectators) to be carried by the hyperon are assumed to have momentum distributions determined from their original structure functions. The polarized sea quarks, carrying the remaining fraction of the hyperon's momentum, combine with the spectator quarks to produce polarization in the hyperon.

These models are approximations of a more complex process and each of these models has difficulties. The Lund Model, as originally proposed, incorrectly predicted the sign of the $\bar{\Lambda}$ polarization from $\mathrm{K}^{+}$production. This was later "fixed", at the cost of introducing extra parameters, by allowing the spectator quarks to flip their spins before combining with sea quarks to form the hyperon [41]. The other models do not require this spin-flip to occur. The D-M Model proposes a mechanism for the polarization and a useful parameterization of the results, but glosses over an explicit numerical calculation of its magnitude. Szwed's model predicts lower polarization than is observed.

Regarding E756, the D-M model predicts that unpolarized protons produce unpolarized $\Omega^{-} s$ [42]. The authors of the other models have not made specific predictions but it is apparent that the Lund model predicts the $\Omega^{-}$s produced in Phase 1 are polarized. The parameterization of the D-M Model predicts that polarized hyperons containing strange quarks will produce polarized $\Omega^{-}$s and $\Xi^{-}$s. Appendix B contains an elaboration of this result. 


\section{Chapter 3}

\section{Apparatus}

The Tevatron at Fermilab produced an $800 \mathrm{GeV} / \mathrm{c}$ primary proton beam. The accelerator operated with a cycle approximately one minute long. The beam spill duration was 20 seconds. This beam was divided and transported to the different experimental beamlines which operated simultaneously during fixed-target running periods. E756 occupied the Proton Center beamline and experimental area. The amount of beam delivered to Proton Center was adjusted by the Tevatron operators to settings depending on E756 operating conditions as discussed below (Section 3.6).

E756 consisted of a beamline designed for transporting $800 \mathrm{GeV} / \mathrm{c}$ protons from the Tevatron to the target, a target area, a curved magnetic channel, and a spectrometer for collecting information on the charged particle tracks. This chapter describes the various parts of the apparatus and how they were used.

\subsection{Beamline and Target Area Enclosure}

The Proton Center beamline was roughly 890 meters long from the entrance of the first enclosure to the end of the spectrometer. It consisted of a series of dipole and quadrupole magnets for steering and focusing the proton beam, several segmented-wireion-chambers (SWICs) for determining the beam's position and a secondary-emission moniter (SEM) for measuring the beam's intensity. The last 46 meters of the beam transport system was the target area, PC3, which contained the magnets for producing the final focus and targeting angle.

The two methods used to produce the $\Omega^{-}$required different magnet and target arrangements in PC3. Figure 3.1 shows the vertical view of PC3 including the path of 
the beam center for Phase 1 and Phase 2 .

During Phase 1, primary protons entered PC3 at 0 milliradian $(\mathrm{mR})$ with no offset, either horizontal or vertical, with respect to the centerline of the target. The first magnet elements in PC3 were a set of quadrupole magnets for providing a vertical beam focus at the target. Following the quadrupoles was a horizontal trim dipole magnet. The beam was then steered upward or downward by 6-3-120 dipole magnets (the nomenclature 6-3-120 indicates the horizontal aperture, the vertical aperture and the length, all in inches). They put the beam in position so that a series of EPB (5-1.5120) dipoles could bend the beam back and produce the desired targeting angle at the target. The main targeting angles used were $\pm 2.5 \mathrm{mR}$ and $0.0 \mathrm{mR}$. The targeting angle and beam position were monitored with SWICs placed at the beginning of the enclosure and in front of the target. Care was taken to maintain the targeting angle within $1 / 4$ $\mathrm{mR}$ of the desired setting. The target, a $2 \times 2 \mathrm{~mm}^{2}$ Be rod of $1 / 4$ interaction length (4 inch), was placed directly in front of the curved magnetic channel entrance. The channel (Figure 3.2) is discussed in the next section. The beam spot size was smaller than the target. A SEM determined the beam intensity.

During Phase 2 , the $\Omega^{-}$were produced by a neutral beam. This neutral beam originated at a 1/2" diameter by 1 interaction length ( 6 inch) $\mathrm{Cu}$ target placed in front of a straight magnetic channel embedded in a B2 dipole magnet. This channel (Figure 3.3) was 21' long and located immediately upstream of the Hyperon Magnet. The first $10.5^{\prime}$ had a $0.25 \times 0.50 \mathrm{inch}^{2}$ aperture. For the second 10.5', the aperture was closed to $1 / 8 \times 1 / 8 \mathrm{inch}^{2}$. Since this neutral beam was produced at either a $+2 \mathrm{mR}$ or $-2 \mathrm{mR}$ targeting angle, the hyperons contained significant polarization. The magnetic field of the neutral particle channel was oriented parallel to this polarization so that the neutral particles polarization did not precess. In order to accomodate this new target and channel, the Phase 1 dipoles were moved further upstream. Since less space was available for making the targeting angle, an additional 6-3-120 and EPB dipole were also required. The polarized neutral beam produced the $\Omega^{-}$at a $0.2 \times 0.2 \mathrm{in}^{2} \times 1$. interaction length ( 6 inch) $\mathrm{Cu}$ target located in front of the curved magnetic channel. 

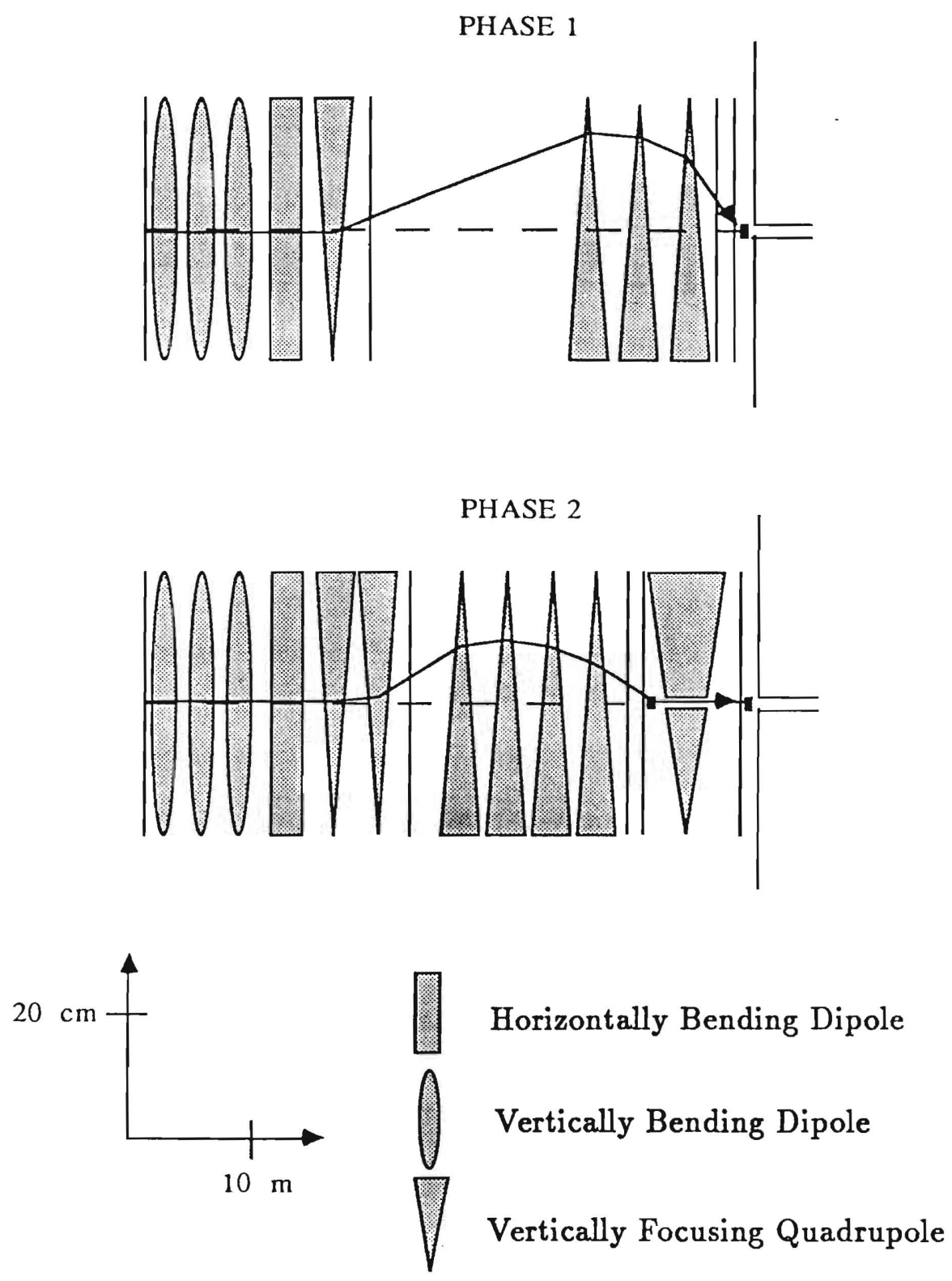

Figure 3.1: The target enclosure, $\mathrm{PC3}$, shown in vertical view for Phase 1 (upper) and Phase 2 (lower). The scales are different for $x$ and $y$. The size of the beam is not shown. 
Again, the beam's position and intensity were monitored by means of SWICS and a SEM.

Figure 3.4 shows the transverse momentum of the $\Xi^{-}$parallel to $\hat{y}$, in the spectrometer coordinate system for Phase 1 compared with Phase 2. Both positive and negative targeting angle are shown. This figure illustrates the difference between Phase 1 and Phase 2. In Phase 1 , the $\Xi^{-}$are produced by a proton beam incident on a target at an angle with respect to the outgoing $\Xi^{-}$. The asymmetry of the Phase 1 distribution shows the the targeting angle changed sign at the production target. In Phase 2, the neutral beam was produced while alternating targeting angles but the $\Xi^{-}$were always produced at $0 \mathrm{mR}$. Thus, the distributions for the two targeting angles are symmetric and peaked at $0 \mathrm{GeV} / \mathrm{c}$.

\subsection{Hyperon Magnet and Charged Particle Channel}

The Proton Center Hyperon Magnet is a 7.31 meter long dipole magnet with a vertical field tunable up to 35 Kgauss (3.5 Tesla). The magnet had an $8 . \times 4$. inch ${ }^{2}$ rectangular hole along the centerline which held a brass channel filled with copper and tungsten blocks. The negative charged beam traversed a curved path arranged in the blocks. Neutral and positive charged particles hit the walls of the collimator. The defining aperture of the path was $0.197 \times 0.197$ inch $^{2}$. The bend angle was $14.7 \mathrm{mR}$. Figure 3.2 shows the location of the aperture and position of the copper and tungsten blocks in the Phase 1 charged particle channel. The Phase 2 charged particle channel was the same one used in Phase 1. Figure 3.3 shows the arrangement of the neutral particle and charged particle channels used in Phase 2.

The Hyperon Magnet current was operated at each of several settings in order to select different kinematic regions and precess the $\Omega^{-}$through different magnetic fields. Table 3.1 shows the field integral for the seven currents used during the experiment. Before data-taking began, the field in the channel was measured at 1-inch intervals with a calibrated Hall probe. This provided the field integrals for four of the fields. The field 


\section{Phase 1}

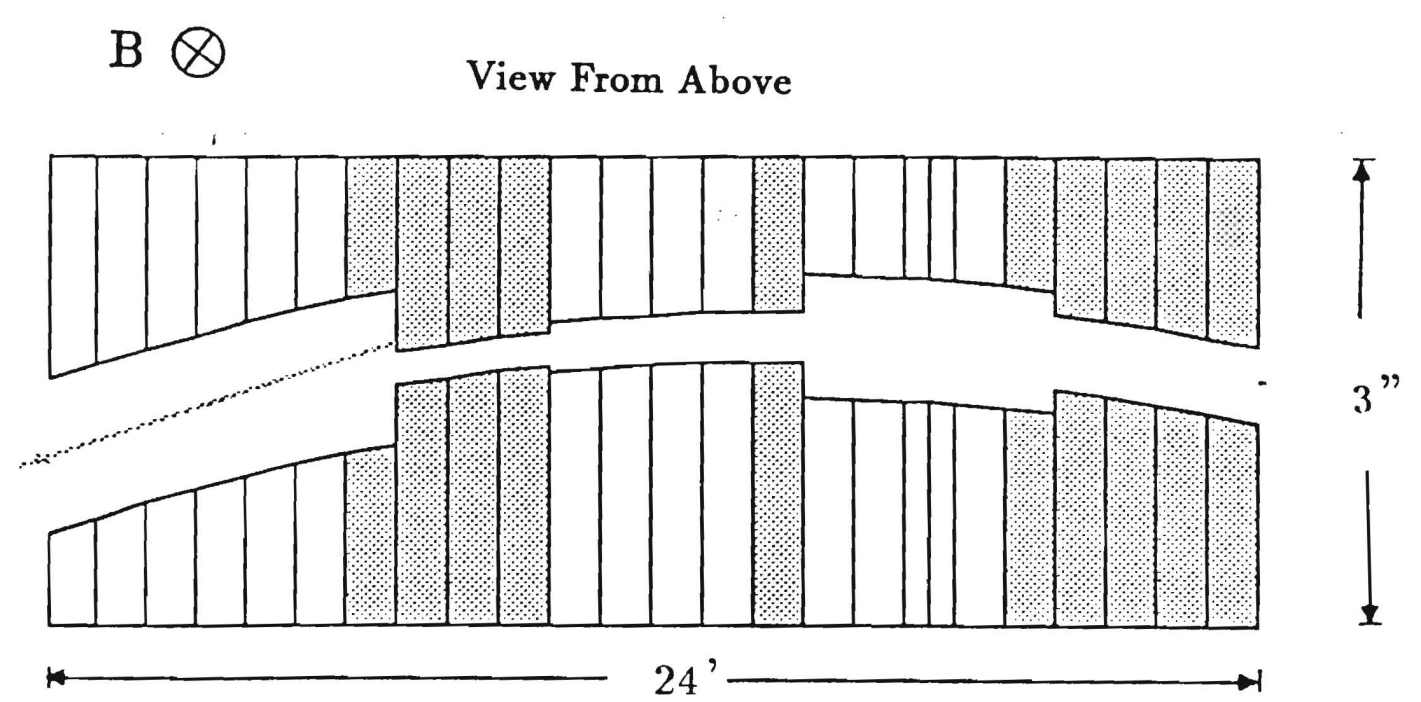

View From the Side

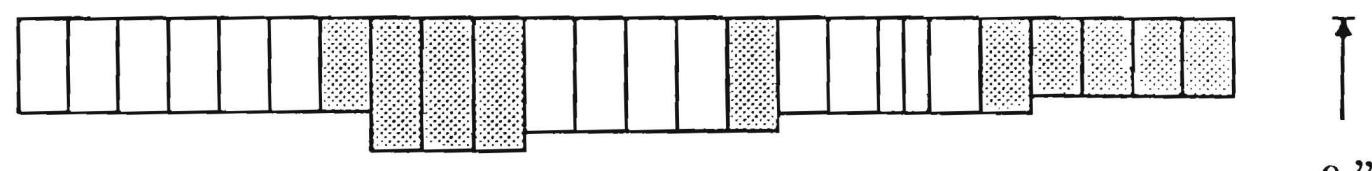

$.9 "$

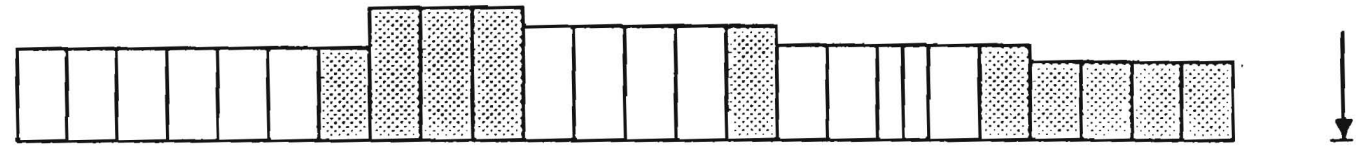

Figure 3.2: Phase 1 collimator. The shaded sections are made of tungsten. The unshaded regions are brass. The views from above (plan) and from the side (elevation) are shown, including the direction of the beam centerline. The magnetic field direction is shown. 


\section{Phase 2}

View From Above

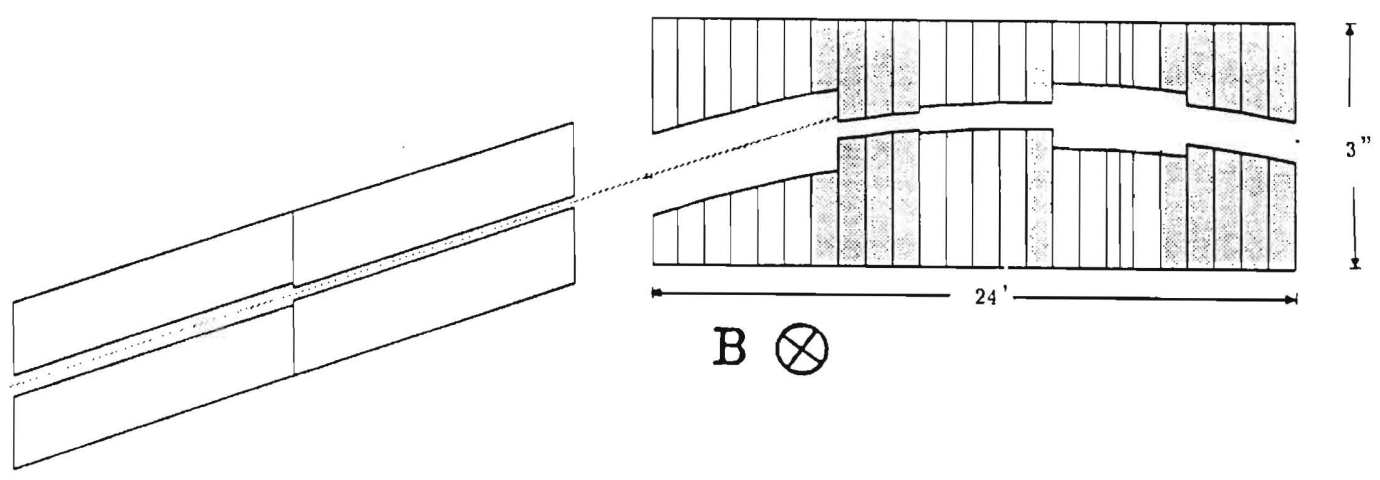

View From the Side

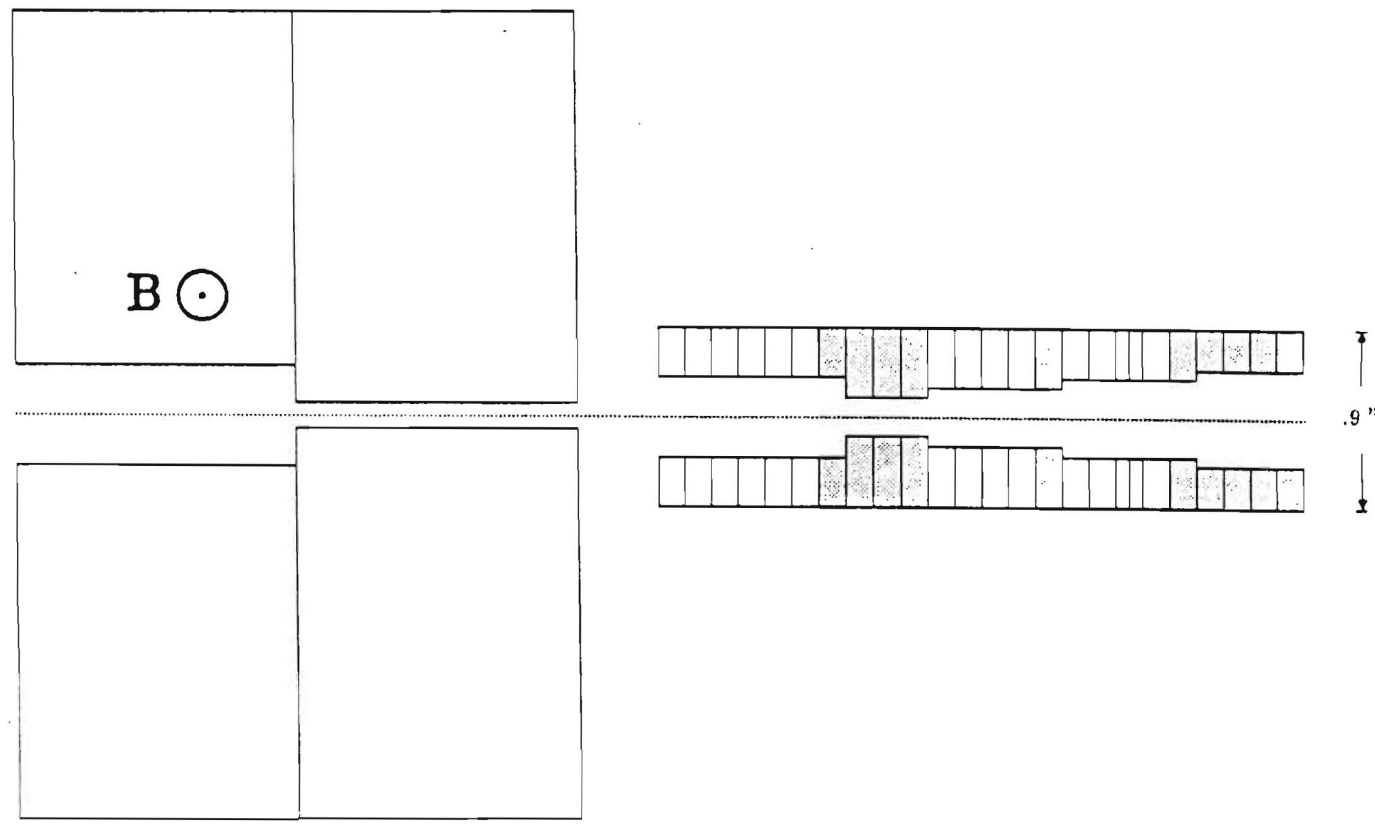

Figure 3.3: Phase 2 collimators. The charged particle channel is the same one used in Phase 1. The plan and elevation views are shown. The shaded sections are made of tungsten. The unshaded regions are brass. The magnetic field directions are shown. 

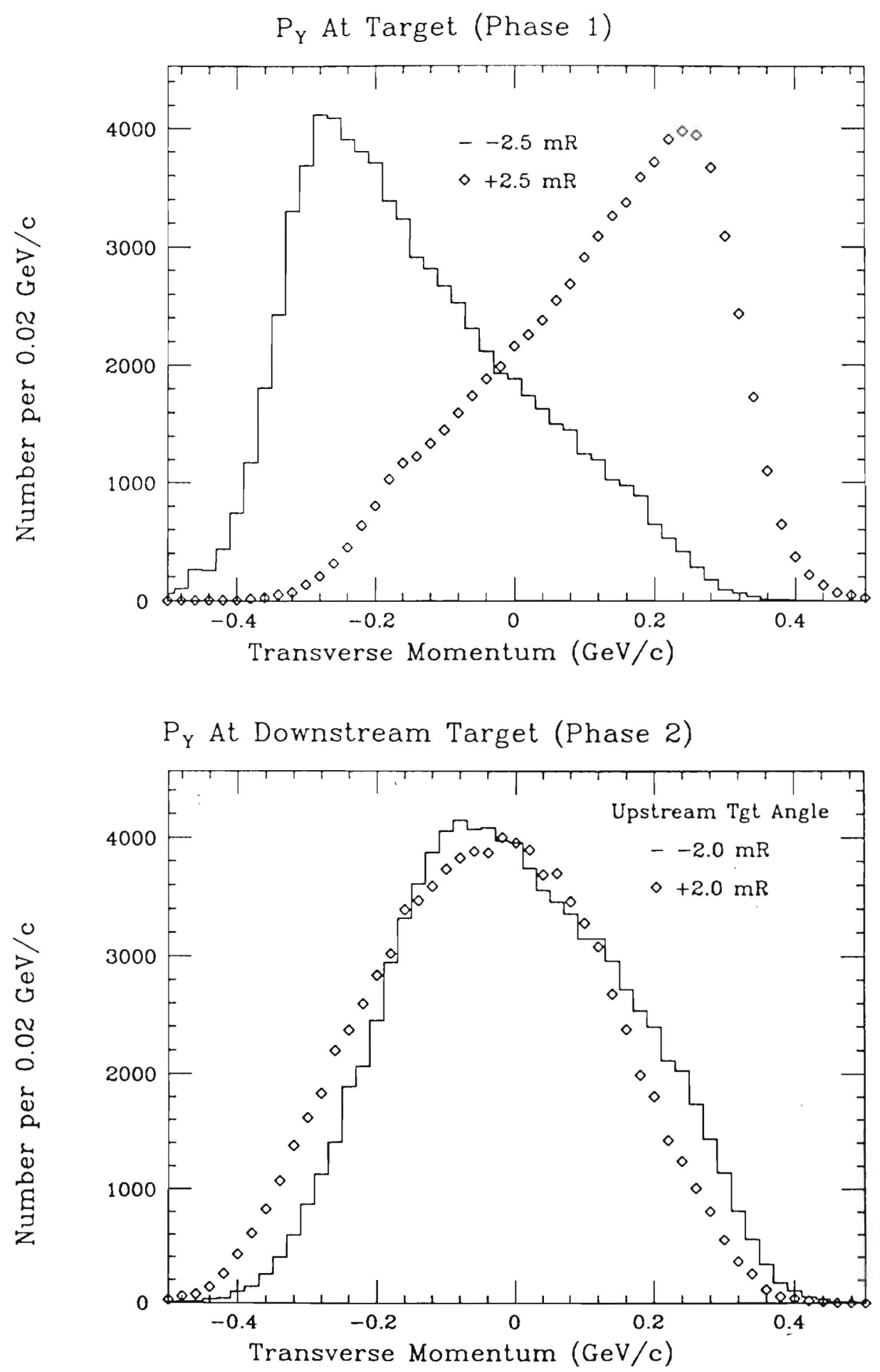

Figure 3.4: $\Xi^{-} P_{y}$ at the production target for Phase 1 and Phase 2. Both positive and negative targeting angles are shown. These $\Xi^{-} s$ were recorded with the Hyperon Magnet current set at -1000 Amps. 


\begin{tabular}{||c|c||}
\hline $\begin{array}{c}\text { Current } \\
\text { (Amps) }\end{array}$ & $\begin{array}{c}\text { Field Integral } \\
\text { (Tesla-meters) }\end{array}$ \\
\hline-450 & -14.48 \\
\hline-500 & -15.30 \\
\hline-750 & -17.87 \\
\hline-1000 & -19.43 \\
\hline-1700 & -22.18 \\
\hline-2500 & -24.11 \\
\hline-3300 & -25.62 \\
\hline
\end{tabular}

Table 3.1: Hyperon Magnet field integral vs. current.

integral of the other currents was estimated from the excitation curve and checked with target-pointing. The estimated uncertainty in the field integrals is less than $1 \%$. When at full field, the Hyperon Magnet and curved channel delivered a beam with a broad momentum spectrum peaked at $500 \mathrm{Gev} / \mathrm{c}$. Figure 3.5 shows the relative channel acceptance as a function of momentum for all seven Hyperon magnet currents for beam pions. The acceptance of $\Omega^{-} \mathrm{s}$ and $\Xi^{-} \mathrm{s}$ is modified by the hyperons exponential decay. During the run, the current was monitored with the beamline controls system and by means of a digital readout connected directly to the magnet current controller.

\subsection{Spectrometer}

The spectrometer is shown in Figure 3.5. Charged particle tracks were detected in a series of silicon-strip detectors (SSDs), multiwire proportional chambers (MWPCs), and scintillation counters. A pair of dipole magnets installed about two-thirds of the way through the spectrometer provided a transverse bend to charged particles so that their momentum could be determined. Available, but not used in this analysis was a hodoscope array and two stacks of lead-glass blocks for electromagnetic calorimetry. A lab coordinate system has been defined in such a way that the charged particles following the central orbit of the Hyperon Magnet moved along the $\hat{z}$ direction. Vertically upward was the $\hat{y}$ direction. The $\hat{x}$ direction was defined so as to make the coordinate system 


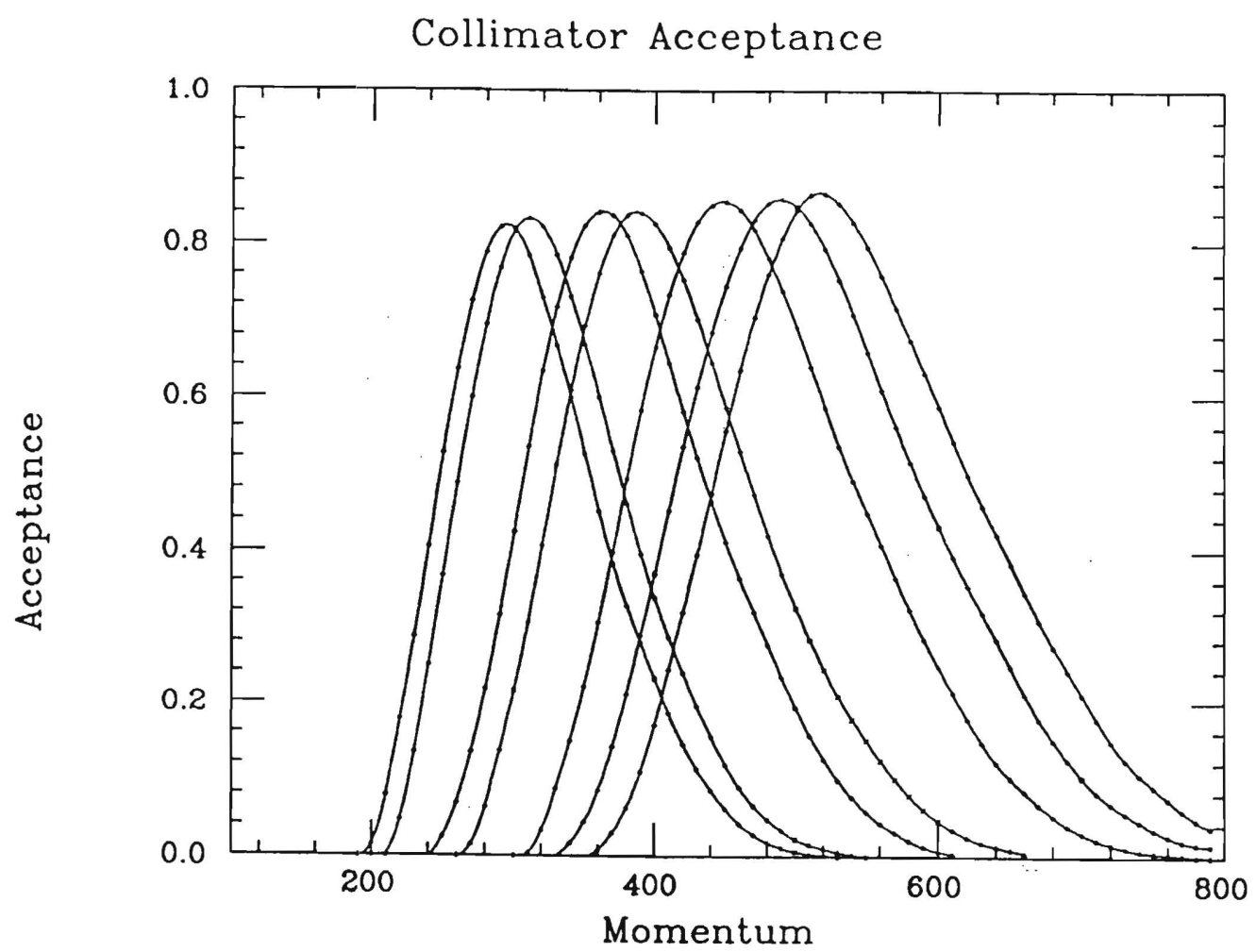

Figure 3.5: Relative charged particle collimator acceptance. This plot shows the acceptance of beam pions for the seven Hyperon Magnet fields used in the experiment. This was determined from a Monte Carlo simulation of the collimator. 
right-handed. This convention will be followed in the remainder of this report.

The SSDs were the first detectors after the Hyperon Magnet exit. There were eight planes, four with the strips parallel to $\hat{x}$ alternating with four with the strips parallel to $\hat{y}$. Each SSD covered a $2.8 \times 2.8 \mathrm{~cm}^{2}$ area with $1 / 10 \mathrm{~mm}$ wide strips. In order to reduce rf noise, they were provided special filtered power and a temperature-controlled rf-sheilded housing.

There were nine MWPCs. All were of the standard design of three cathode (highvoltage) planes sandwiching two orthogonal anode (signal-wire) planes. The signal wires were oriented along $\hat{x}$ and $\hat{y}$ with the exception of chamber C5, whose signal wires were rotated 45 degrees with respect to the others, and chamber $\mathrm{C} 6$ which had a fourth high-voltage plane and a third signal plane, also rotated by 45 degrees. Table 3.2 shows the construction parameters including the spacing (pitch), tension and diameter of the anode and cathode planes. The half gaps for both the $1 \mathrm{~mm}$ and $2 \mathrm{~mm}$ MWPCs were 3/16 inch. Table Table 3.3 shows the size of the MWPCs, their sense wire spacing, and their locations with respect to the channel exit. All of the chambers used a gas mixture containing, by weight, $99.88 \%$ argon and $0.12 \%$ freon which was bubbled through a jar of liquid methylal at 2 degrees centigrade. During the run the MWPC's performance was monitored both on-line and off-line and by the current drawn from the chamber power supplies. Table 3.3 also shows their average particle detection efficiency.

$\mathrm{S} 1, \mathrm{~S} 2, \cdot \mathrm{V} 1$, and V2 were thin scintillation counters. S1, $1.5 \times 2.5 \mathrm{inch}^{2}$, and S2, 4.5 x 2.5 inch $^{2}$, covered the beam spot at their respective positions. V1 and V2 were "halo" counters which covered the near beam region and surrounded, but did not cover, the beam. V1 was $12.75 \times 3.5$ inch $^{2}$ with a $4.25 \times 2.5$ inch $^{2}$ hole removed from the center. S2 fit over this hole. V2 was $16.5 \times 4.5$ inch $^{2}$ with a $5.5 \times 3.25$ inch $^{2}$ hole. The halo counters detected very low momentum particles and muons near the beam.

$M$ was a $1 / 2$ inch thick scintillation counter placed in the beam to count the chargedparticle multiplicity of each event. The scintillating material was a special high-lightyield plastic, NE110. A multichannel analyzer was used to determine the range of 

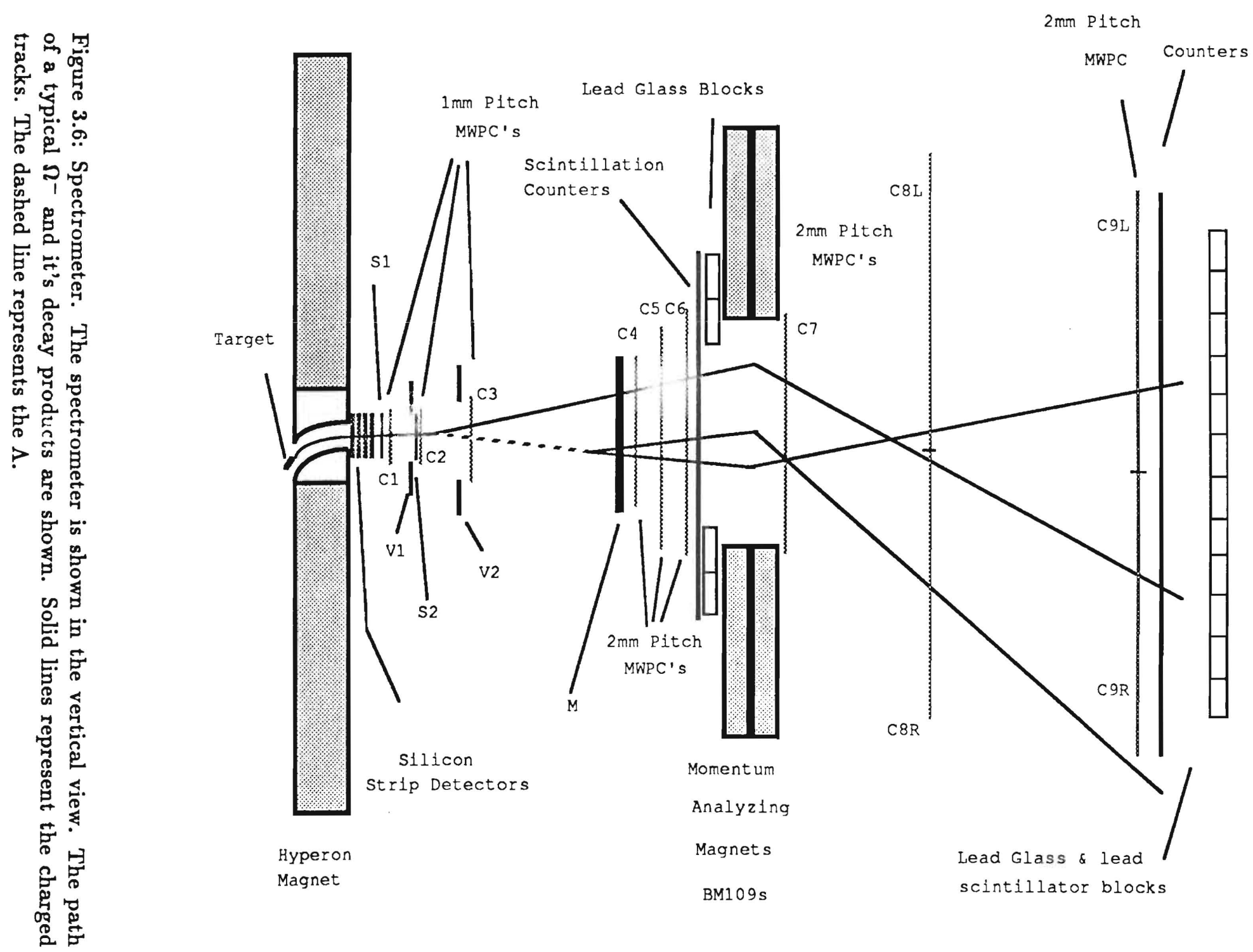


\begin{tabular}{||c|c|c|c|c|c|c||}
\hline & \multicolumn{2}{|c|}{$\begin{array}{c}\text { (Pitch) } \\
(\mathrm{mm})\end{array}$} & \multicolumn{2}{c|}{$\begin{array}{c}\text { Wire Diameter } \\
\text { (mils) }\end{array}$} & \multicolumn{2}{c|}{$\begin{array}{c}\text { Tension } \\
\text { (g-wt) } \\
\text { Anode }\end{array}$} \\
MWPC & Anode & Cathode & Anode & Cathode & Anode & Cathode \\
\hline C1 & 1. & 0.5 & 0.8 & 2.0 & 50. & 100. \\
\hline C2 & 1. & 0.5 & 0.8 & 2.0 & 50. & 100. \\
\hline C3 & 1. & 0.5 & 0.8 & 2.0 & 50. & 100. \\
\hline C4 & 2. & 1.0 & 1.0 & 2.0 & 75. & 100. \\
\hline C5 & 2. & 1.0 & 1.0 & 2.0 & 75. & 100. \\
\hline C6 & 2. & 1.0 & 1.0 & 2.0 & 75. & 100. \\
& 2.828 & & 1.0 & & 75. & \\
\hline C7 & 2. & 1.0 & 1.0 & 2.0 & 75. & 100. \\
\hline C8 & 2. & 1.0 & 1.0 & 2.0 & 75. & 100. \\
\hline C9 & 2. & 1.0 & 1.0 & 2.0 & 75 & 100. \\
\hline
\end{tabular}

Table 3.2: MWPC construction characteristics. Cathode refers to the high voltage wires; anode refers to the sense wires. The tension is given in terms of the weight applied to each wire. ( $1 \mathrm{mil}=0.001$ inch $=25.4$ microns.)

\begin{tabular}{||c|c|c|c|c|c||}
\hline MWPC & $\begin{array}{c}\text { Anode Pitch } \\
(\mathrm{mm})\end{array}$ & View & $\begin{array}{c}\text { Size } \\
\left(\mathrm{cm}^{2}\right)\end{array}$ & $\begin{array}{c}\text { Location } \\
(\mathrm{cm})\end{array}$ & $\begin{array}{c}\text { Average } \\
\text { Efficiency }\end{array}$ \\
\hline C1 & 1. & $\mathrm{x}-\mathrm{y}$ & $12.8 \times 12.8$ & 406. & .965 \\
\hline C2 & 1. & $\mathrm{x}-\mathrm{y}$ & $25.6 \times 25.6$ & 755. & .976 \\
\hline C3 & 1. & $\mathrm{x}-\mathrm{y}$ & $25.6 \times 25.6$ & 1056. & .612 \\
\hline C4 & 2. & $\mathrm{x}-\mathrm{y}$ & $51.2 \times 25.6$ & 2598. & .989 \\
\hline C5 & 2. & $\mathrm{v}-\mathrm{u}$ & $51.2 \times 51.2$ & 3155. & .969 \\
\hline C6 & 2. & $\mathrm{x}-\mathrm{y}$ & $51.2 \times 51.2$ & 3607. & .990 \\
\cline { 2 - 3 } & 2.828 & $\mathrm{u}$ & & & .973 \\
\hline C7 & 2. & $\mathrm{x}-\mathrm{y}$ & $63.8 \times 25.6$ & 4311. & .952 \\
\hline C8 & 2. & $\mathrm{x}-\mathrm{y}$ & $128 . \times 38.4$ & 4898. & .983 \\
\hline C9 & 2. & $\mathrm{x}-\mathrm{y}$ & $128 . \times 38.4$ & 6229. & .973 \\
\hline
\end{tabular}

Table 3.3: MWPC characteristics. The location is the distance from the collimator exit of the center of the chamber along $\hat{z}$. The efficiency is the average of the $\mathrm{x}$ and $\mathrm{y}$ planes over the whole data sample. 
signals from an RCA-8850 photo-multiplier for single, double, triple, and more-thantriple coincident charged-particle hits. The multiplicity counter signal for each event was read out by an analog-digital converter (ADC) and monitored during Phase 2. The average ADC signal was insensitive to the particles $x-y$ position at the counter.

The momentum-analyzing magnets were two 6-foot-long dipole magnets (BM109s) with vertical fields and mirror plates on the ends. The field of the first magnet was of 15.7 Kgauss. The aperture was $24 \times 10$ inch $^{2}$. The field of the second magnet was 11.4 Kgauss. It's aperture was $24 \times 12$ inch $^{2}$. Before startup, field maps were made which showed that the magnetic fields, apart from the fringe fields at the magnet ends, were uniform to better than $0.5 \%$. A longitudinal section of the field map is shown for the center of the two magnets in Figure 3.3. The net effect of the fields was to introduce a $1.556 \mathrm{Gev} / \mathrm{c}$ transverse kick to charged particles passing through the magnets. The direction of the field of the magnets was reversed for some of the conditions in Phase 1 (see Tables 3.6 and 3.7 for more specific information).

Since the magnets separated particles according to their charges, the prompt signals from the sense wires of each half, left and right, of C8 and C9 were combined in an "or"-circuit with the division at the chamber center. These chambers were installed so that a $\mathrm{K}^{-}$from an $\Omega^{-}$decay or a $\pi^{-}$from a $\Lambda$ decay would hit the left or right side of C8 (depending on the BM109 field polarity) and protons from the $\Lambda$ decay would hit the opposite side of C9.

Helium filled polyethylene bags with thin windows were placed in between the chambers and counters and inside the BM109s to reduce the effects of multiple coulomb scattering of the charged particles.

\subsection{Trigger}

Triggers were of two types. The "charged-particle" trigger required coincident hits in scintillation counters S1 and S2 as well as no hits in the halo counters V1 or V2. Thus,

$$
T_{\text {chg }} \equiv S 1 \cdot S 2 \cdot \overline{V 1} \cdot \overline{V 2}
$$


BY VS Z

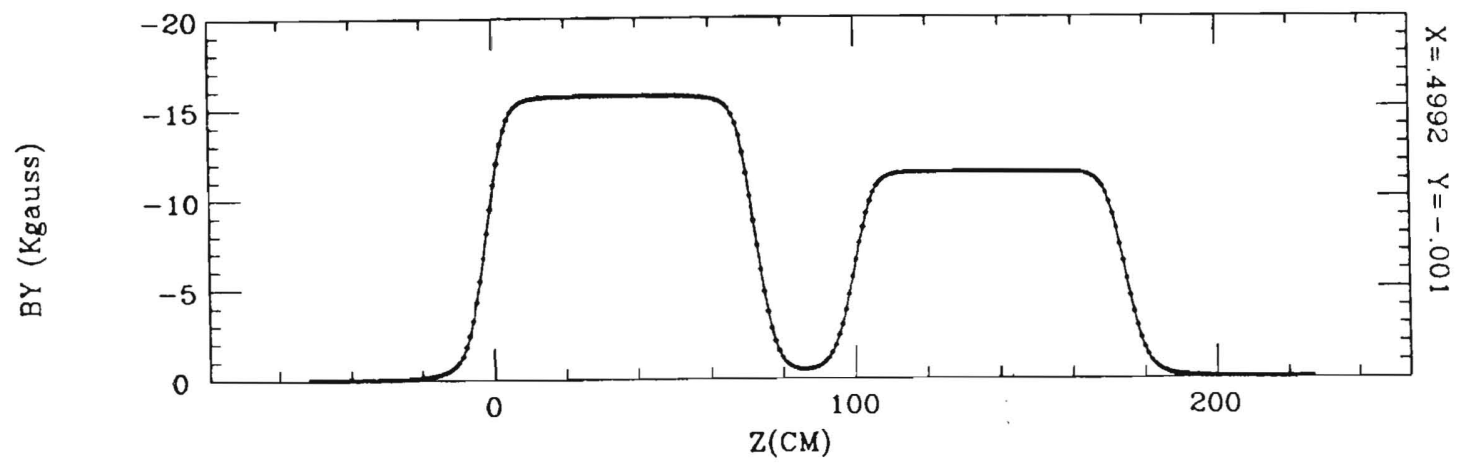

BX VS Z

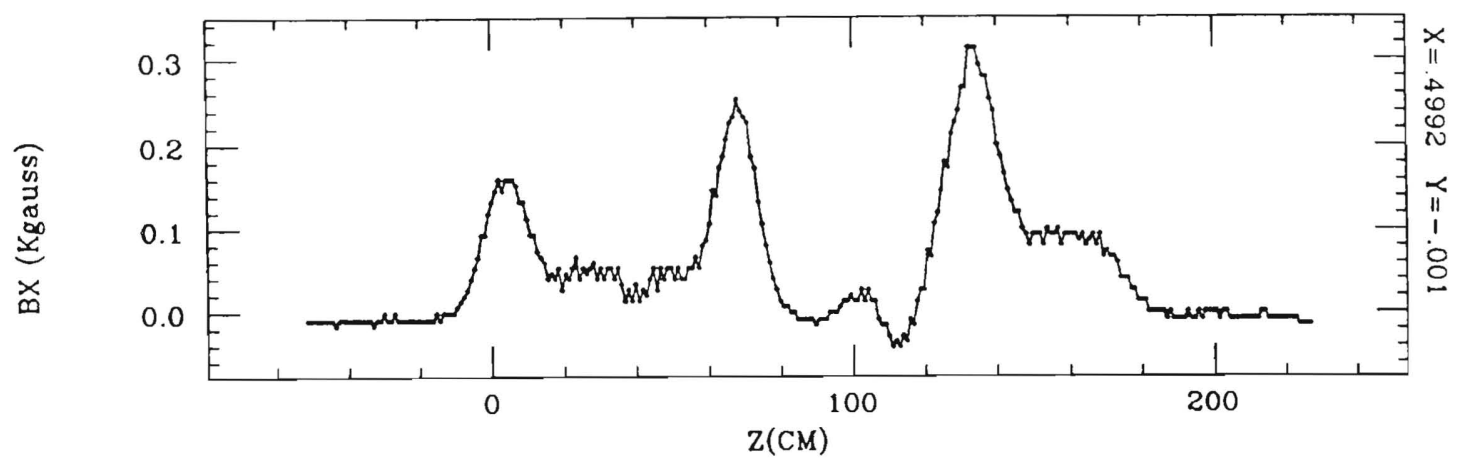

BZ VS Z

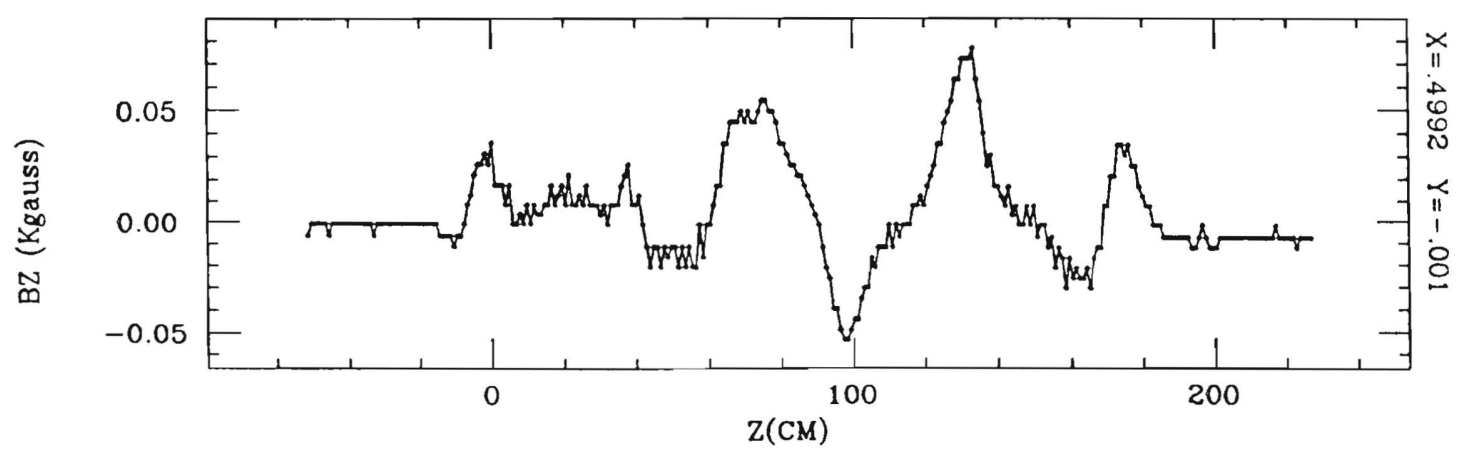

Figure 3.7: Field of the BM109s. The plots show $B_{y}, B_{x}$, and $B_{z}$, respectively, of both BM109s. The horizontal scale is distance along the center of the magnets in $\mathrm{cm}$. The vertical scale is the field in Kgauss. The right hand side is the $\mathrm{x}-\mathrm{y}$ position in $\mathrm{cm}$. 
indicated the presence of a charged particle, usually a $\pi^{-}$, in the spectrometer. The "three-track" trigger required the presence of the charged-particle trigger coincident with at least one hit on the negative charge side of $\mathrm{C} 8$, at least one hit on the positive charge side of $\mathrm{C} 9$, and a signal, $\mathrm{M}$, from the multiplicity counter with a peak voltage in the three-charged-hit range. The signal from the multiplicity counter was split and put into two discriminators set at $285 \mathrm{mV}$ and $450 \mathrm{mV}$. The trigger required the output from the discriminator set at $285 \mathrm{mV}$ was "true" and the output from the discriminator set at $450 \mathrm{mV}$ was "not true". Essentially, the trigger tried to select three-chargedparticle events containing at least one negatively charged and one positively charged particle from a beam containing mostly single negatively charged particle events. Thus,

$$
T_{3-t r k} \equiv T_{c h g} \cdot C 8_{n e g} \cdot C 9_{p o s} \cdot \bar{M}_{>450 m V} \cdot M_{>285 m V}
$$

Due to the identical decay topologies, the three-track trigger could not distinguish $\Omega^{-} \rightarrow$ $\Lambda \mathrm{K}^{-}$from $\Xi^{-} \rightarrow \Lambda \pi^{-}$decays. A prescaled fraction of the $T_{c h g}$ triggers was recorded for calibration purposes with the three-track data as well as on special calibration tapes.

\subsection{Data Acquisition}

When an event satisfied the trigger, several signals were generated. The trigger sent a timed "good event" signal to the MWPCs, SSDs, and latches to hold the hit wires, strips and latchable counter information. A timed gate signal was sent to the ADC modules. A "busy signal" held off additional triggers. Then a priority interrupt sent to a PDP-11-45 initiated the RT11-MULTI read-out process through a PDP-CAMAC interface. The record for each event contained the event header, six latch words, MWPC data, SSD data and ADC information. The full readout for a typical event took about $70 \mu$ sec. The events were sent to a memory buffer, then written onto magnetic tape.

A second type of priority interrupt was generated before the beginning and after the end of each spill which recorded ADC pedestals and the accumulated scalers. 


\subsection{Rates and Tapes}

The number of protons/spill requested from the Tevatron was determined from the singles rate of $\mathrm{C} 4$ which could not exceed about 800 kilohertz. This rate as well as the three-track and single track trigger rates depended on the targeting angle and Hyperon Magnet field. Tables 3.4 and 3.5 show the ungated rates for the major targeting angles and Hyperon Magnet settings during Phase 1 and Phase 2. For the high-rate conditions of Phase 1, the trigger rate was limited by both $\mathrm{C} 4$ and the computer-busy signal. During Phase 2, the intensity of the tertiary beam was much less that the chamber rate limit. The triggers were limited by the number of protons available from the Tevatron for Proton-Center. 


\begin{tabular}{|c|c|c|c|c|c|c|c|}
\hline $\begin{array}{c}\text { Hyperon } \\
\text { Magnet } \\
\text { (Amps) }\end{array}$ & $\begin{array}{r}\text { BM109s } \\
\text { (Amps) }\end{array}$ & $\begin{array}{l}\text { Vert. } \\
\text { Tgting } \\
\text { Angles } \\
(\mathrm{mR})\end{array}$ & $\begin{array}{c}\text { Protons } \\
\text { per } \\
\text { Spill } \\
\times 10^{10} \\
\end{array}$ & $\begin{array}{c}\text { Ungated } \\
\text { 3-Track } \\
\text { Triggers } \\
\text { per } \\
\text { Proton } \\
\times 10^{-8} \\
\end{array}$ & $\begin{array}{c}\text { Ungated } \\
1 \text {-Track } \\
\text { Triggers } \\
\text { per } \\
\text { Proton } \\
\times 10^{-5} \\
\end{array}$ & $\begin{array}{c}\text { C4 Rate } \\
(\mathrm{Khz})\end{array}$ & $\begin{array}{c}\text { Trigger } \\
\text { Livetime }\end{array}$ \\
\hline \multirow[t]{2}{*}{-500} & -2500 & \pm 2.5 & 2.4 & 54 & 19 & 530 & 0.50 \\
\hline & 2500 & \pm 2.5 & 1.2 & 61 & 21 & 313 & 0.64 \\
\hline \multirow[t]{2}{*}{-1000} & -2500 & \pm 2.5 & 0.9 & 24 & 7.8 & 140 & 0.82 \\
\hline & 2500 & \pm 2.5 & 3.1 & 34 & 11 & 562 & 0.59 \\
\hline \multirow[t]{4}{*}{-1700} & \multirow[t]{2}{*}{-2500} & \pm 2.5 & 2.8 & 16 & 5.6 & 286 & 0.77 \\
\hline & & +0.0 & 1.3 & 197 & 53 & 449 & 0.35 \\
\hline & \multirow[t]{2}{*}{2500} & \pm 2.5 & 2.2 & 17 & 5.8 & 403 & 0.78 \\
\hline & & +0.0 & 1.0 & 148 & 51 & 564 & 0.47 \\
\hline \multirow[t]{4}{*}{-2500} & \multirow[t]{2}{*}{-2500} & \pm 2.5 & 3.5 & 9.7 & 4.2 & 456 & 0.81 \\
\hline & & +0.0 & 1.4 & 137 & 39 & 656 & 0.44 \\
\hline & \multirow[t]{2}{*}{2500} & \pm 2.5 & 2.8 & 11 & 4.1 & 308 & 0.81 \\
\hline & & +0.0 & 1.5 & 124 & 39 & 536 & 0.41 \\
\hline \multirow[t]{4}{*}{-3300} & \multirow[t]{2}{*}{-2500} & \pm 2.5 & 4.0 & 6.5 & 3.4 & 395 & 0.86 \\
\hline & & +0.0 & 1.6 & 108 & 31 & 634 & 0.45 \\
\hline & \multirow[t]{2}{*}{2500} & \pm 2.5 & 4.5 & 11 & 3.6 & 530 & 0.81 \\
\hline & & +0.0 & 1.1 & 92 & 17 & 436 & 0.53 \\
\hline
\end{tabular}

Table 3.4: Phase 1. Typical rates for each beam condition. Main conditions. The rates/proton for target out conditions are typically 50 times lower. 


\begin{tabular}{|c|c|c|c|c|c|c|c|}
\hline $\begin{array}{c}\text { Hyperon } \\
\text { Magnet } \\
\text { (Amps) }\end{array}$ & $\begin{array}{c}\text { Upstrm. } \\
\text { Tgting } \\
\text { (mR) }\end{array}$ & $\begin{array}{c}\text { Downst. } \\
\text { Tgting } \\
0.0 \mathrm{mR}\end{array}$ & $\begin{array}{c}\text { Protons } \\
\text { per } \\
\text { Spill } \\
\mathbf{x} 10^{10} \\
\end{array}$ & $\begin{array}{c}\text { Ungated } \\
\text { 3-Track } \\
\text { Triggers } \\
\text { per } \\
\text { Proton } \\
\mathbf{x} 10^{-10} \\
\end{array}$ & $\begin{array}{c}\text { Ungated } \\
\text { 1-Track } \\
\text { Triggers } \\
\text { per } \\
\text { Proton } \\
\mathbf{x} 10^{-8} \\
\end{array}$ & $\begin{array}{c}\text { C4 } \\
\text { Rate } \\
(\mathrm{Khz}) \\
\end{array}$ & $\begin{array}{c}\text { Trigger } \\
\text { Livetime }\end{array}$ \\
\hline \multirow[t]{4}{*}{-450} & \pm 2.0 & \multirow[t]{2}{*}{ (Tgt In) } & 53 & 3.9 & 22 & 117 & 0.88 \\
\hline & 0.0 & & 64 & 94 & 306 & 185 & 0.67 \\
\hline & \pm 2 . & \multirow[t]{2}{*}{ (Out) } & 62 & 1.8 & 28 & 205 & 0.85 \\
\hline & 0.0 & & 47 & 41 & 115 & 231 & 0.52 \\
\hline \multirow[t]{2}{*}{-750} & \pm 2.0 & \multirow[t]{2}{*}{ (Tgt In) } & 58 & 2.4 & 12 & 101 & $\overline{0.94}$ \\
\hline & +0.0 & & 55 & 81 & 216 & 190 & 0.71 \\
\hline \multirow[t]{3}{*}{-1000} & \pm 2.0 & \multirow[t]{3}{*}{ (Tgt In) } & 59 & 1.8 & 8.7 & 88 & 0.96 \\
\hline & \pm 1.0 & & 55 & 20 & 37 & 106 & 0.78 \\
\hline & +0.0 & & 49 & 68 & 168 & 150 & $0 . \overline{76}$ \\
\hline
\end{tabular}

Table 3.5: Phase 2. Typical rates. 
Each tape was divided into 2 to 4 runs, took between one and three hours to record and held a mixture of about 400,000 one-track and three-track triggers. The pre-scale factor for the one-track trigger was set as high as possible so long as the one-track triggers did not to intrude on the three track triggers through the trigger busy signal. During Phase 1 it was normally set at $1 / 2048$. During Phase 2 , it was normally set at $1 / 64$. Tables 3.6 to 3.8 show the number of tapes, runs and triggers for the main conditions used in Phase 1 and Phase 2. 


\begin{tabular}{|c|c|c|c|c|c|c|}
\hline $\begin{array}{c}\text { Hyperon } \\
\text { Magnet } \\
\text { (Amps) }\end{array}$ & $\begin{array}{l}\text { BM109s } \\
\text { (Amps) }\end{array}$ & $\begin{array}{c}\text { Vertical } \\
\text { Targeting } \\
\text { Angles (mR) }\end{array}$ & Tapes & Runs & $\begin{array}{c}\text { Three } \\
\text { Track } \\
\text { Triggers }\end{array}$ & $\begin{array}{c}\text { One } \\
\text { Track } \\
\text { Triggers }\end{array}$ \\
\hline \multirow[t]{4}{*}{-500} & \multirow[t]{2}{*}{-2500} & \multirow{4}{*}{$\begin{array}{c}-2.5 \\
+2.5 \\
-2.5 \\
+2.5\end{array}$} & 8 & 19 & 2266585 & 389376 \\
\hline & & & 8 & 19 & 2458193 & 430583 \\
\hline & \multirow[t]{2}{*}{2500} & & 7 & 14 & 2306630 & 419682 \\
\hline & & & 9 & 18 & 2979725 & 555597 \\
\hline \multirow[t]{4}{*}{-1000} & \multirow[t]{2}{*}{-2500} & \multirow{4}{*}{$\begin{array}{l}-2.5 \\
+2.5 \\
-2.5 \\
+2.5\end{array}$} & 18 & 46 & 6823707 & 108354 \\
\hline & & & 19 & 50 & 7322839 & 116182 \\
\hline & \multirow[t]{2}{*}{2500} & & 12 & 23 & 4226413 & 807970 \\
\hline & & & 11 & 23 & 3893146 & 702115 \\
\hline \multirow[t]{6}{*}{-1700} & \multirow[t]{3}{*}{-2500} & \multirow{6}{*}{$\begin{array}{l}-2.5 \\
+2.5 \\
+0.0 \\
-2.5 \\
+2.5 \\
+0.0\end{array}$} & 13 & 35 & 3974765 & 127570 \\
\hline & & & 11 & 31 & 3542979 & 122167 \\
\hline & & & 2 & 4 & 736328 & 110961 \\
\hline & \multirow[t]{3}{*}{2500} & & 7 & 17 & 2186152 & 405801 \\
\hline & & & 6 & 16 & 2213523 & 414604 \\
\hline & & & 2 & 4 & 679968 & 126124 \\
\hline \multirow[t]{6}{*}{-2500} & \multirow[t]{3}{*}{-2500} & \multirow{6}{*}{$\begin{array}{l}-2.5 \\
+2.5 \\
+0.0 \\
-2.5 \\
+2.5 \\
+0.0\end{array}$} & 3 & 6 & 955411 & 205417 \\
\hline & & & 3 & 6 & 950799 & 211610 \\
\hline & & & 2 & 4 & 701032 & 107654 \\
\hline & \multirow[t]{3}{*}{2500} & & 2 & 4 & 629572 & 140078 \\
\hline & & & 2 & 4 & 495480 & 102182 \\
\hline & & & 2 & 4 & 664205 & 117283 \\
\hline \multirow[t]{6}{*}{-3300} & \multirow[t]{3}{*}{-2500} & -2.5 & 5 & 18 & 1728657 & 0 \\
\hline & & +2.5 & 4 & 13 & 1187766 & 0 \\
\hline & & +0.0 & 2 & 4 & 716243 & 112325 \\
\hline & \multirow[t]{3}{*}{2500} & -2.5 & 2 & 7 & 619260 & 64251 \\
\hline & & +2.5 & 2 & 7 & 622588 & 61468 \\
\hline & & +0.0 & 2 & 4 & 662632 & 115185 \\
\hline
\end{tabular}

Table 3.6: Phase 1. Number of tapes for each beam condition. Main conditions. There were $\sim 55.5 \mathrm{M}$ (million) 3 -trk triggers recorded among these conditions. 


\begin{tabular}{|c|c|c|c|c|c|c|}
\hline $\begin{array}{l}\text { Hyperon } \\
\text { Magnet } \\
\text { (Amps) }\end{array}$ & $\begin{array}{l}\text { BM109s } \\
\text { (Amps) }\end{array}$ & $\begin{array}{c}\text { Horizontal } \\
\text { Targeting } \\
\text { Angles (mR) }\end{array}$ & Tapes & Runs & $\begin{array}{c}\text { Three } \\
\text { Track } \\
\text { Triggers }\end{array}$ & $\begin{array}{c}\text { One } \\
\text { Track } \\
\text { Triggers }\end{array}$ \\
\hline \multirow[t]{2}{*}{-1000} & -2500 & \multirow{2}{*}{$\begin{array}{c}\text { (Tgt Out) }+0.0 \\
+0.0\end{array}$} & 1 & 2 & 320035 & 64265 \\
\hline & 2500 & & 1 & 2 & 317859 & 68087 \\
\hline \multirow[t]{10}{*}{-1700} & -2500 & \multirow{5}{*}{$\begin{array}{c}\text { (Tgt Out) }+0.0 \\
+0.0 \\
-1.3 \\
-1.3 \\
+1.3\end{array}$} & 1 & 2 & 326666 & 66004 \\
\hline & 2500 & & 1 & 2 & 317767 & 66003 \\
\hline & -2500 & & 1 & 2 & 324467 & 62043 \\
\hline & \multirow[t]{2}{*}{2500} & & 1 & 2 & 319293 & 69047 \\
\hline & & & 1 & 2 & 348269 & 64935 \\
\hline & \multirow[t]{3}{*}{-2500} & \multirow{5}{*}{$\begin{array}{c}\text { (Tgt In) }-1.3 \\
+1.3 \\
-2.2 \\
-1.3 \\
+1.3\end{array}$} & 1 & 2 & 345427 & 54741 \\
\hline & & & 1 & 2 & 332454 & 66972 \\
\hline & & & 1 & 1 & 179180 & 28896 \\
\hline & \multirow[t]{2}{*}{2500} & & 1 & 2 & 332972 & 59734 \\
\hline & & & 1 & 2 & 338938 & 65834 \\
\hline \multirow[t]{10}{*}{-2500} & -2500 & \multirow{7}{*}{$\begin{array}{c}(\text { Tgt Out })+0.0 \\
+0.0\end{array}$} & 1 & 2 & 318738 & 61214 \\
\hline & 2500 & & 1 & 2 & 323551 & 65686 \\
\hline & \multirow[t]{2}{*}{-2500} & & 1 & 2 & 329421 & 63787 \\
\hline & & & 1 & 2 & 331486 & 97016 \\
\hline & \multirow[t]{2}{*}{2500} & & 1 & 2 & 322117 & 68616 \\
\hline & & & 1 & 2 & 369473 & 74810 \\
\hline & \multirow[t]{2}{*}{-2500} & & 1 & 2 & 335923 & 53402 \\
\hline & & \multirow{3}{*}{$\begin{array}{c}\text { (Tgt In) }-1.3 \\
+1.3 \\
-1.3 \\
+1.3\end{array}$} & 1 & 2 & 329027 & 77568 \\
\hline & \multirow[t]{2}{*}{2500} & & 1 & 2 & 338722 & 60261 \\
\hline & & & 1 & 2 & 332706 & 73929 \\
\hline \multirow[t]{14}{*}{-3300} & -2500 & \multirow{6}{*}{$\begin{array}{c}\text { (Tgt Out) }+0.0 \\
+0.0 \\
-1.3 \\
+1.3 \\
-1.3 \\
+1.3 \\
\end{array}$} & 1 & 2 & 318672 & 62619 \\
\hline & 2500 & & 1 & 2 & 315514 & 64340 \\
\hline & \multirow[t]{2}{*}{-2500} & & 2 & 2 & 324603 & 66924 \\
\hline & & & 1 & 2 & 314116 & 95468 \\
\hline & \multirow[t]{2}{*}{2500} & & 1 & 2 & 314358 & 64742 \\
\hline & & & 1 & 2 & $\mathbf{3 3 4 9 1 7}$ & 66625 \\
\hline & \multirow[t]{4}{*}{-2500} & \multirow{8}{*}{$\begin{array}{c}\text { (Tgt In) }-2.2 \\
-1.3 \\
+1.3 \\
+2.2 \\
-2.2 \\
-1.3 \\
+1.3 \\
+2.2\end{array}$} & 1 & 1 & 80752 & 13994 \\
\hline & & & 1 & 2 & 342378 & 54839 \\
\hline & & & 1 & 4 & 334102 & 96390 \\
\hline & & & 2 & 5 & 357428 & 151677 \\
\hline & \multirow[t]{4}{*}{2500} & & 1 & 1 & 82370 & 13734 \\
\hline & & & 1 & 2 & 333886 & 59434 \\
\hline & & & 1 & 2 & 353517 & 80480 \\
\hline & & & 1 & 1 & 74913 & 18555 \\
\hline
\end{tabular}

Table 3.7: Phase 1. Number of tapes for each beam condition. Odd conditions. The target in or target out labels apply to all the conditions within their respective boxes. There were $\sim 11.0 \mathrm{M} 3$-trk triggers recorded among these conditions. 


\begin{tabular}{|c|c|c|c|c|c|c|c|}
\hline $\begin{array}{l}\text { Hyperon } \\
\text { Magnet } \\
\text { (Amps) }\end{array}$ & $\begin{array}{l}\text { BM109s } \\
\text { (Amps) }\end{array}$ & $\begin{array}{c}\text { Upstrm. } \\
\text { Tgting } \\
\text { (mR) }\end{array}$ & $\begin{array}{c}\text { Downstm. } \\
\text { Tgting } \\
0.0 \mathrm{mR}\end{array}$ & Tapes & Runs & $\begin{array}{c}\text { Three } \\
\text { Track } \\
\text { Triggers }\end{array}$ & $\begin{array}{c}\text { One } \\
\text { Track } \\
\text { Triggers }\end{array}$ \\
\hline \multirow[t]{6}{*}{-450} & \multirow[t]{6}{*}{-2500} & -2.0 & \multirow[t]{3}{*}{ (Tgt In) } & 83 & 180 & 3397093 & 30343518 \\
\hline & & +2.0 & & 69 & 151 & 3026299 & 25523290 \\
\hline & & +0.0 & & 21 & 42 & 4379992 & 3534407 \\
\hline & & -2.0 & \multirow[t]{3}{*}{ (Tgt Out) } & 3 & 9 & 157066 & 1621851 \\
\hline & & +2.0 & & 4 & 12 & 69761 & 2277142 \\
\hline & & +0.0 & & 3 & 8 & 224483 & 1572546 \\
\hline \multirow[t]{3}{*}{-750} & \multirow[t]{3}{*}{-2500} & -2.0 & \multirow[t]{3}{*}{ (Tgt In) } & 1 & 3 & 61929 & 512786 \\
\hline & & +2.0 & & 1 & 3 & 63926 & 529084 \\
\hline & & +0.0 & & 1 & 2 & 246891 & 145671 \\
\hline \multirow[t]{5}{*}{-1000} & \multirow[t]{5}{*}{-2500} & -2.0 & \multirow[t]{5}{*}{ (Tgt In) } & 5 & 14 & 256005 & 1893531 \\
\hline & & -1.0 & & 1 & 3 & 119152 & 477385 \\
\hline & & +1.0 & & 1 & 3 & 129611 & 464709 \\
\hline & & +2.0 & & 5 & 13 & 282649 & 2252635 \\
\hline & & +0.0 & & 1 & 2 & 243718 & 134939 \\
\hline
\end{tabular}

Table 3.8: Phase 2. Number of tapes for each beam condition. There were $\sim 12.7 \mathrm{M}$ 3-trk triggers recorded among these conditions. 


\section{Chapter 4}

\section{Event Analysis}

The event analysis consisted of computer programs written to analyze the data tapes recorded during the experiment. The tapes were analyzed one run at a time. Each event in the run was treated sequentially and classified according to it's quality and the likelyhood that it was an $\Omega^{-}$. Straight-line tracks were formed from the hit wires and strips of the MWPCs and SSDs. Events with three tracks were fitted to the two vertex, three track decay topology of the $\Omega^{-} \rightarrow \Lambda \mathrm{K}^{-}$or $\Xi^{-} \rightarrow \Lambda \pi^{-}$, where the $\Lambda \rightarrow \mathrm{p} \pi^{-}$decay chain. A kinematic fit was then performed on events which survived the geometric fit to determine if a $\Lambda$ could be identified among the three tracks. Finally, the remaining track was assumed to be a $\mathrm{K}^{-}$or $\pi^{-}$and the mass of the primary was determined. If the primary's mass was close to the $\Omega^{-}$mass, but not close to the $\Xi^{-}$mass, the event was deemed an $\Omega^{-}$candidate. $\Omega^{-}$and $\Xi^{-}$candidates were stored in "tapout files" containing the reconstructed momenta and vertices. These files were then analyzed to locate a clean $\Omega^{-}$and $\Xi^{-}$sample. A series of selection criteria called "cuts" removed events from the sample which were reconstructed poorly or were more likely decays other than $\Omega^{-} \rightarrow \Lambda \mathrm{K}^{-}$or $\Xi^{-} \rightarrow \Lambda \pi^{-}$.

This chapter concentrates on the reconstruction algorithm and the event selection used in choosing the $\Omega^{-}$and $\Xi^{-}$samples. The first section is a detailed discussion of the track-fitting and event reconstruction. The next section discusses the "Monte Carlo" model of the experiment used for studying the spectrometer acceptance, the performance of the reconstruction program and the polarization analysis. The third section contains a discussion of the $\Omega^{-}$event selection and background. The final section contains a discussion of the $\Xi^{-}$event selection and background. 


\subsection{Reconstruction}

The first part of the reconstruction program was the tracking routine. The goal of the track-finder was to identify three track events and sort the hits in the MWPCs and SSDs according to their track. First, the track-finder counted the number of hits in each SSD and MWPC and converted the hits into $\mathrm{x}$ and $\mathrm{y}$ coordinates. Events were rejected if the number of hits in C4-C9 did not satisfy the minimal requirements necessary for the tracking program to find three tracks. Rather than the strict requirement of three separate hits in each view of every chamber: at least three out of the four planes of $\mathrm{C} 4$ and $\mathrm{C} 5$ had two or more hits; at least three out of the five planes of C5U and C6Y to $\mathrm{C} 9 \mathrm{Y}$ had less than four hits and more than one hit; the planes of $\mathrm{C} 5 \mathrm{U}, \mathrm{C} 6 \mathrm{U}$ and C5V each had three or more hits; each $\hat{x}$ plane of C6 to C8 had at least three hits; and, at least two of the $\hat{y}$ planes of $\mathrm{C} 4, \mathrm{C} 7, \mathrm{C} 8$ and $\mathrm{C} 5 \mathrm{U}$ had two or three hits. These hit-counting requirements were loose enough to avoid throwing away good events and tight enough to avoid bogging down the tracking with unreconstructable ones.

The events were then reconstructed using only $\mathrm{C} 4$ to $\mathrm{C} 9$; the hits from the SSDs and $\mathrm{C} 1-\mathrm{C} 3$ were added to the tracks after the event was determined to be a three track candidate. Treating the views independently, the reconstruction program attempted to find three straight-line tracks in the $\hat{y}$ view and three tracks in the $\hat{x}$ view with a bend at the BM109s. Events passed this stage if three tracks were found in the $\hat{y}$ view with hits in at least three chambers, three tracks were found in the $\hat{x}$ view with at least two hits on each track downstream of the spectrometer magnet, and the $\hat{x}$ view tracks could be associated with the $\hat{y}$ view tracks through the $\hat{u}$ planes of $\mathrm{C} 5$ and $\mathrm{C} 6$.

The tracks were then identified as positively or negatively charged particles from the direction of the bend in the BM109s. Two vertices were determined from the distances of closest approach of the tracks. The positively charged track and a negatively charged track were required to form a vertex downstream of their parent particle's intercept with the third track. A least $\chi^{2}$ geometric fit which re-determined the slopes and two decay vertices was performed on the hits. Various combinations of the $\hat{x}$ tracks upstream and 
downstream of the spectrometer magnet and various associations of the $\hat{x}$ and $\hat{y}$ tracks were applied until either the three charged particle, two decay vertex topology with the best fit was found or the possibilities exhausted and the event discarded. If the $\chi^{2}$ was less than 130 for $\sim 30$ degrees of freedom, the event was considered a three track candidate.

The hits in the MWPCs and SSDs upstream of C4 but downstream of the $\Lambda$ vertex were then added to the tracks. Starting with C3 and working upstream one detector at a time, each hit, if found within $3 \mathrm{~mm}$ of a track in $\mathrm{C} 3-\mathrm{C} 1$ or within $1 \mathrm{~mm}$ of a track in the SSDs, was added to the track. For each successive hit candidate, a new geometric fit was performed. The hit was accepted if the $\chi^{2}$ increased by less than 10 compared to the $\chi^{2}$ without the hit and less than 100 in total.

For the sake of the discussion, the particles which made up the downstream vertex are referred to as a proton and a $\pi^{-}$. The third track is referred to as a $\mathrm{K}^{-}$.

The hits in the chambers and SSDs upstream of the $\mathrm{p}-\pi$ vertex were then added to the $\mathrm{K}^{-}$track. If the primary vertex, as determined by the three track geometric fit, was more than $70 \mathrm{~cm}$ downstream of the SSDs, a least-squares fit of the $\mathrm{K}^{-}$track to a straight line with a kink determined which hits belonged on the $\mathrm{K}^{-}$track. If no vertex could be determined this way, if the SSDs did not have at least three hits in both the $\hat{x}$ and $\hat{y}$ views, or if the primary vertex was less than $70 \mathrm{~cm}$ downstream of the SSDs, hits between the two vertices were added to the $\mathrm{K}^{-}$track if the $\chi^{2}$ of a least-squares fit of the $\mathrm{K}^{-}$track to a straight line improved with the hit or was less than 2 per degree of freedom. After the hits on the $\mathrm{K}^{-}$track were found, the three track geometric fit was redone. If the $\chi^{2}$ was greater than 100 , one-at-a-time, the upstream-most hit remaining on the $\mathrm{K}^{-}$track was removed and the fit redone until the $\chi^{2}$ was less than 100 . Finally, events with a $\chi^{2}$ less than 130 were accepted as 3 -track candidates. Figure 4.1 shows the geometric $\chi^{2}$ of the three track candidates for an average of 32 degrees of freedom from a small sample (one run) of the data. 
The next part of the reconstruction program was the kinematic fitter which determined the position of the $\Lambda$ vertex and daughter particle momenta. First, the momentum components of the three tracks were determined from the slopes of the tracks and the bend angles in the spectrometer magnet. The two charged particles making up the downstream vertex were assumed to be a proton and a pion. The proton-pion invariant mass was found by conservation of energy and momentum at the $\Lambda$ vertex. Then a kinematic fit of the $p$ and $\pi^{-}$tracks, with the known $\Lambda$ mass used as a constraint, was performed which determined the final slopes and vertex. Some 3-track candidates were discarded because the kinematic fitter could find no convergent solution. The kinematic $\chi^{2}$ and $\mathrm{p}-\pi^{-}$invariant mass distribution for the three track events of one run are shown in Figure 4.2 and Figure 4.3. This was the end of the tracking and fitting part of the reconstruction program. All events which survived to this point were catagorized "good" three track events. Table 4.1 shows the reconstruction categories and the fraction of events which fell into each for the whole data sample. 


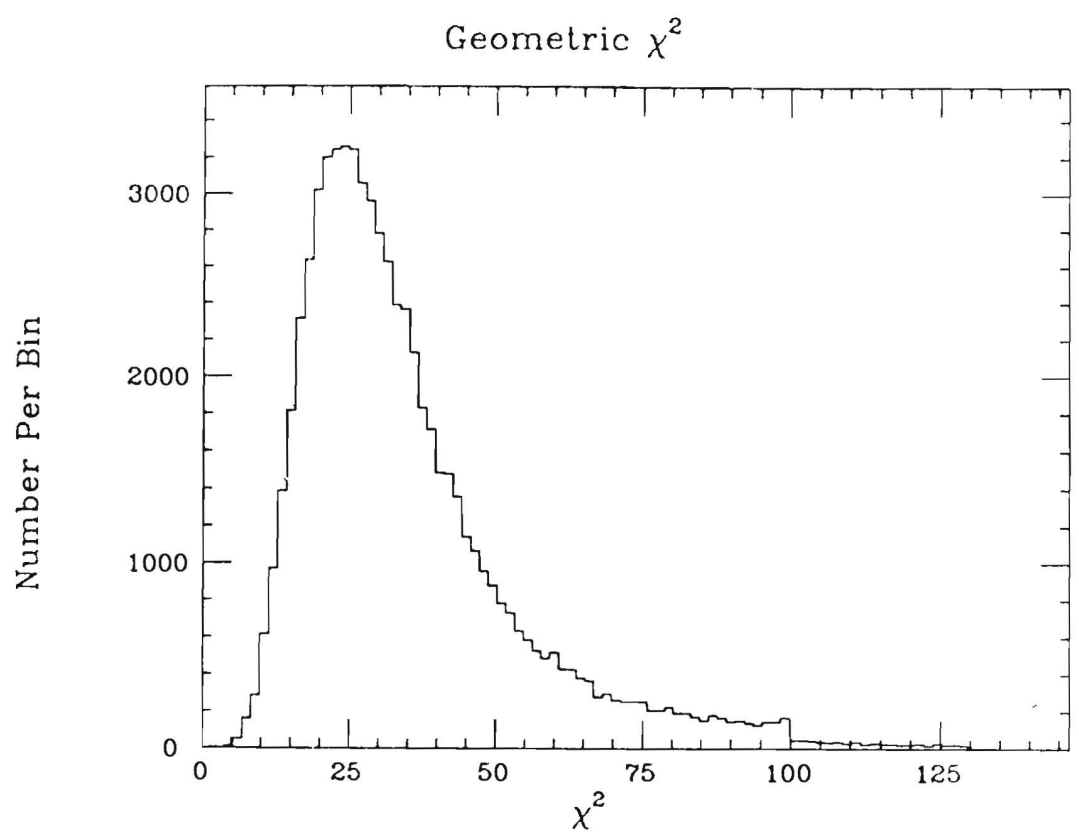

Figure 4.1: Geometric $\chi^{2}$ of the good three track events of a small sample of the data. This includes $\Xi^{-}$events, $\Omega^{-}$events and all of the backgrounds.

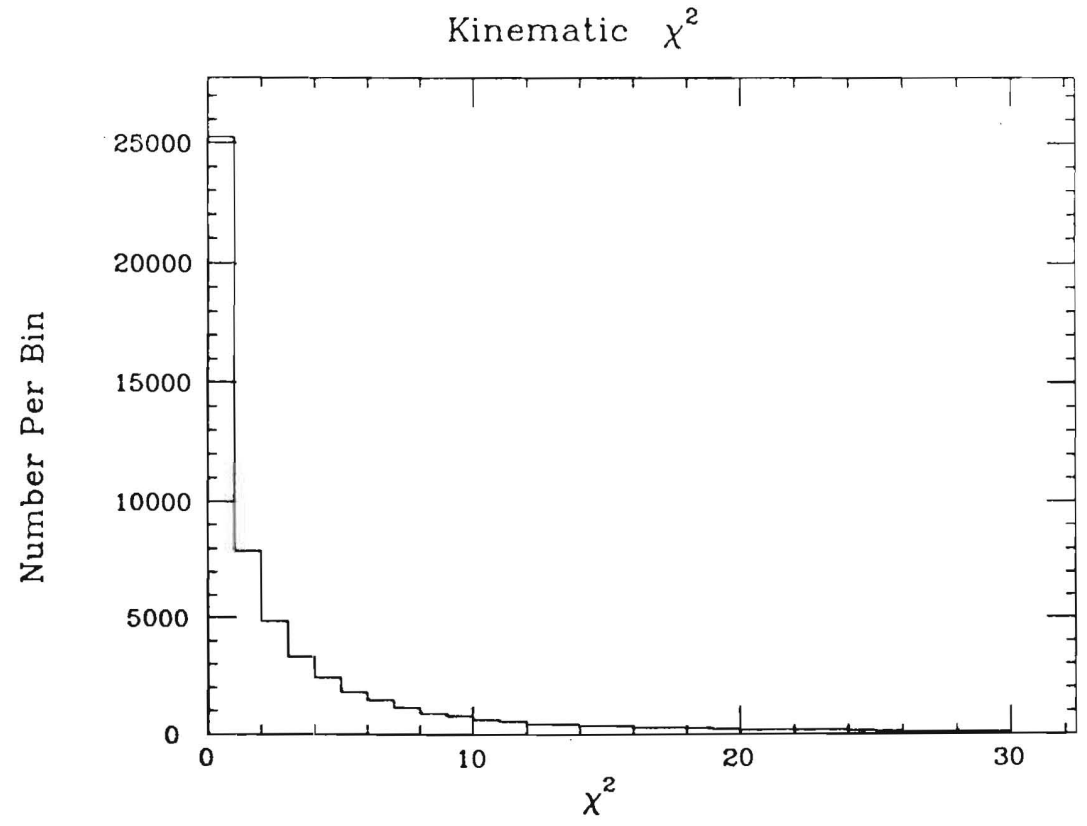

Figure 4.2: Kinematic $\chi^{2}$ of the good three track events of a small sample of the data. This includes $\Xi^{-}$events, $\Omega^{-}$events and their backgrounds. 


\begin{tabular}{|c|c|c|}
\hline $\mathrm{IOK}=$ & Description & $\%$ of Data \\
\hline 0 & Good 3-trk Event & 24.9 \\
\hline 1 & $\begin{array}{l}\text { 3-trk Event in upstrm. Only } 1 \text { hit on } 1 \text { of dnstrm. } \\
\text { trks in } x \text {-view (usually a very low momentum } \pi^{-} \text {). }\end{array}$ & 1.8 \\
\hline 2 & $\begin{array}{l}\text { Less than } 2 \text { hits in }>2 / 4 \text { of planes of } \mathrm{C} 4 \text { and } \mathrm{C} 5 \\
\text { (likely a } 1 \text {-trk trigger checked for a } 3 \text {-trk event). }\end{array}$ & 13.8 \\
\hline 3 & Four or more hits in $>3 / 6$ of the planes of $\mathrm{C} 4-\mathrm{C} 9$. & 4.2 \\
\hline 4 & Two or less hits in $>3 / 6$ of the planes of C4-C9 & 2.6 \\
\hline 5 & $<2$ of $\mathrm{Y}$-planes $\mathrm{C} 4$ and $\mathrm{C} 6-\mathrm{C} 9$ had 2 or 3 hits. & 15.7 \\
\hline 6 & $<3$ points on a $\mathrm{y}$-view track in $\mathrm{C} 4-\mathrm{C} 9$ & 5.5 \\
\hline 7 & $\begin{array}{l}\text { Found } 3 \text { trks in } y \text {-view; found }<3 \text { tracks in } \\
x \text {-view (track finder was confused). }\end{array}$ & 1.1 \\
\hline 8 & $\begin{array}{l}3 \text { tracks before BM109s, } 2 \text { tracks after (likely } \\
\text { a particle hit an aperture at or near the magnet). }\end{array}$ & 2.1 \\
\hline 9 & $\begin{array}{l}\text { The stiffest track bent the same way as a soft } \\
\text { track (track finder was confused). }\end{array}$ & 2.4 \\
\hline 10 & $\begin{array}{l}\text { Two track event in y-view (probably only a } \Lambda \\
\text { from a collimator interaction or early } 3 \text {-trk decay). }\end{array}$ & 6.3 \\
\hline 11 & $\Lambda$ vertex upstr. of primary vertex after Gfit. & 0.8 \\
\hline 12 & $\chi^{2}$ in geometric fitter (Gfit) $>130$ & 2.5 \\
\hline 13 & Gfit does not converge in 10 iterations. & 2.5 \\
\hline 14 & Computer precision error in Gfit. & 0.0 \\
\hline 15 & Kinematic fitter did not converge in 10 iterations. & 0.7 \\
\hline 16 & $\begin{array}{l}\text { Two of the dnstrm. tracks were the same or slope } \\
\text { not found. }\end{array}$ & 1.3 \\
\hline 17 & Cases of IOK $=7$ which deserved extra attention. & 4.8 \\
\hline 18 & Cases of $I O K=8$ which deserved extra attention. & 6.1 \\
\hline 19 & Cases of $I O K=9$ which deserved extra attention. & 2.2 \\
\hline
\end{tabular}

Table 4.1: Reconstruction catagories. Only IOK $=0$ events passed the reconstruction algorithm and were classified "good" three track events. These are compiled from the whole data sample. 


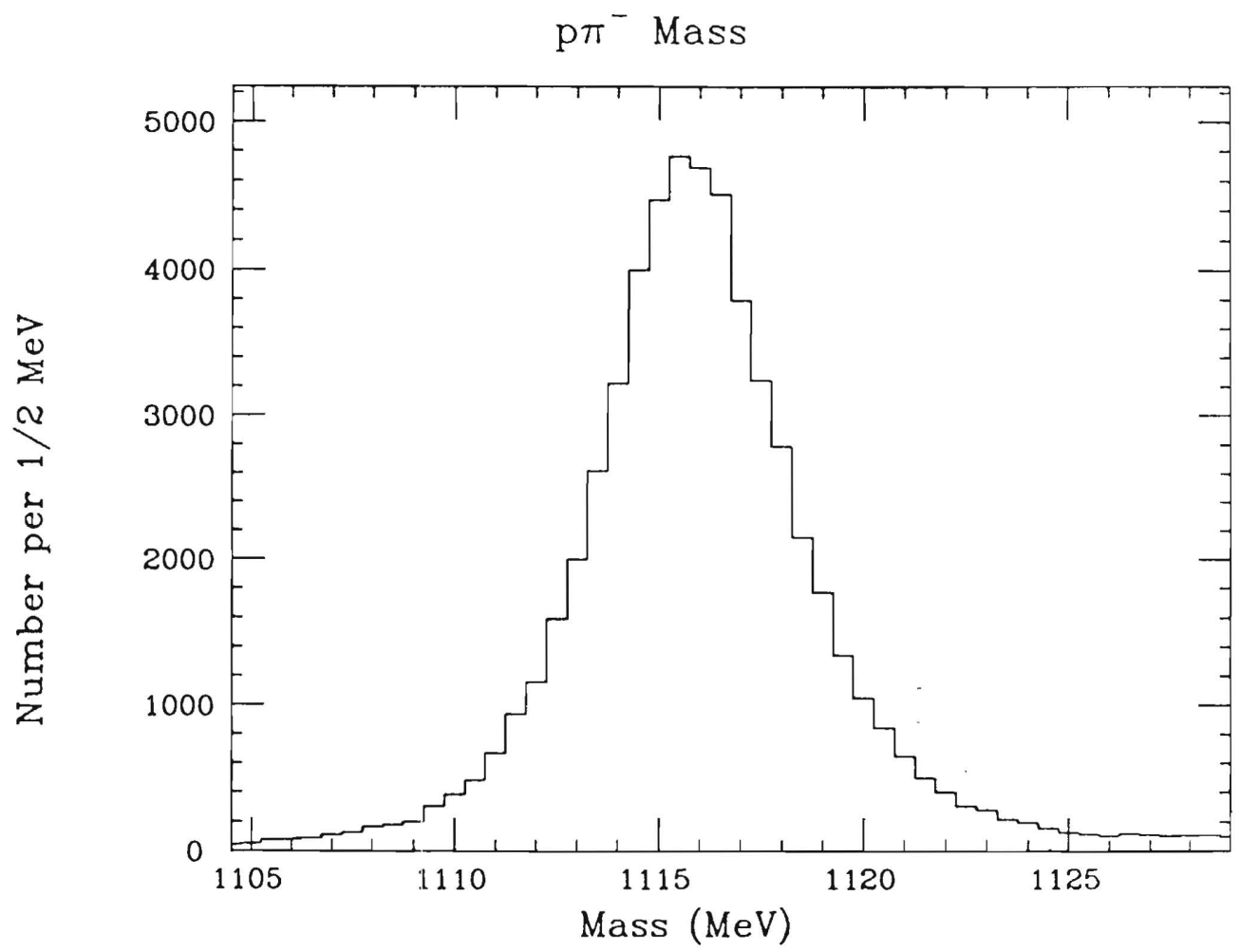

Figure 4.3: Proton- $\pi^{-}$invariant mass for the good three track events of a small sample of the data. This also includes both $\Xi^{-}$events, $\Omega^{-}$events and their backgrounds. 
Until this point, no attempt had been made to separate the $\Omega^{-}$candidates from this sample of mostly $\Xi^{-}$events. The $\Lambda \mathrm{K}^{-}$and $\Lambda \pi^{-}$invariant masses were both calculated from conservation of energy and momentum at the upstream vertex assuming the remaining negative-charged track was either a kaon or a pion, respectively. Figures 4.4 and 4.5 show the $\Lambda \pi^{-}$and $\Lambda \mathrm{K}^{-}$invariant mass plots from one run ( $\sim 1 / 2$ tape). The $\Omega^{-}$mass peak stands out without applying any background cuts. Events for which the $\Lambda \mathrm{K}$ mass was between 1.63 and $1.73 \mathrm{GeV} / \mathrm{c}^{2}$ and the $\Lambda \pi^{-}$mass was not between 1.308 and $1.340 \mathrm{GeV} / \mathrm{c}^{2}$ were considered $\Omega^{-}$candidates. All of the good three track events were classified $\Xi^{-}$candidates. This was the first "cut" which separated $\Omega^{-}$from $\Xi^{-}$events. It will be discussed in more detail later in this chapter.

Data summary files were made for each run. Information for each good event including the momentum vectors of the three charged tracks, the locations of the two vertices, the kinematic and geometric chi-squares, and the $\Lambda$ mass was then written to a data summary file of good $\Omega^{-}$candidates. All of the good three track events were written to a larger file of $\Xi^{-}$candidates. The unpacked raw data for three track events and some one track events was written to a third file. Also, a fourth file containing the final gated and ungated scaler sums and the number of events in each reconstruction category was produced. These "tapout" files were the end product of the raw data reconstruction. They were used in the remainder of the analysis to decide on event selection and in analyzing polarization. 


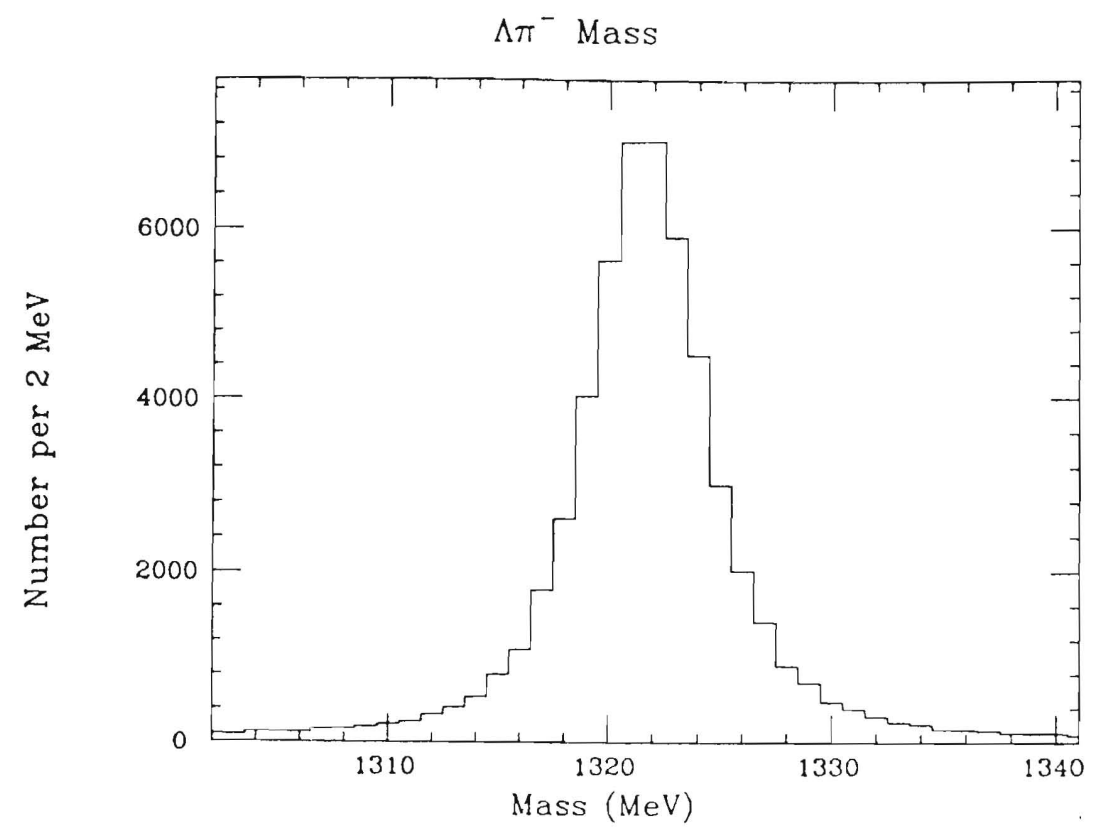

Figure 4.4: $\Lambda-\pi^{-}$invariant mass from "good" 3 -track events. This small sample of the data comes from 1 run.

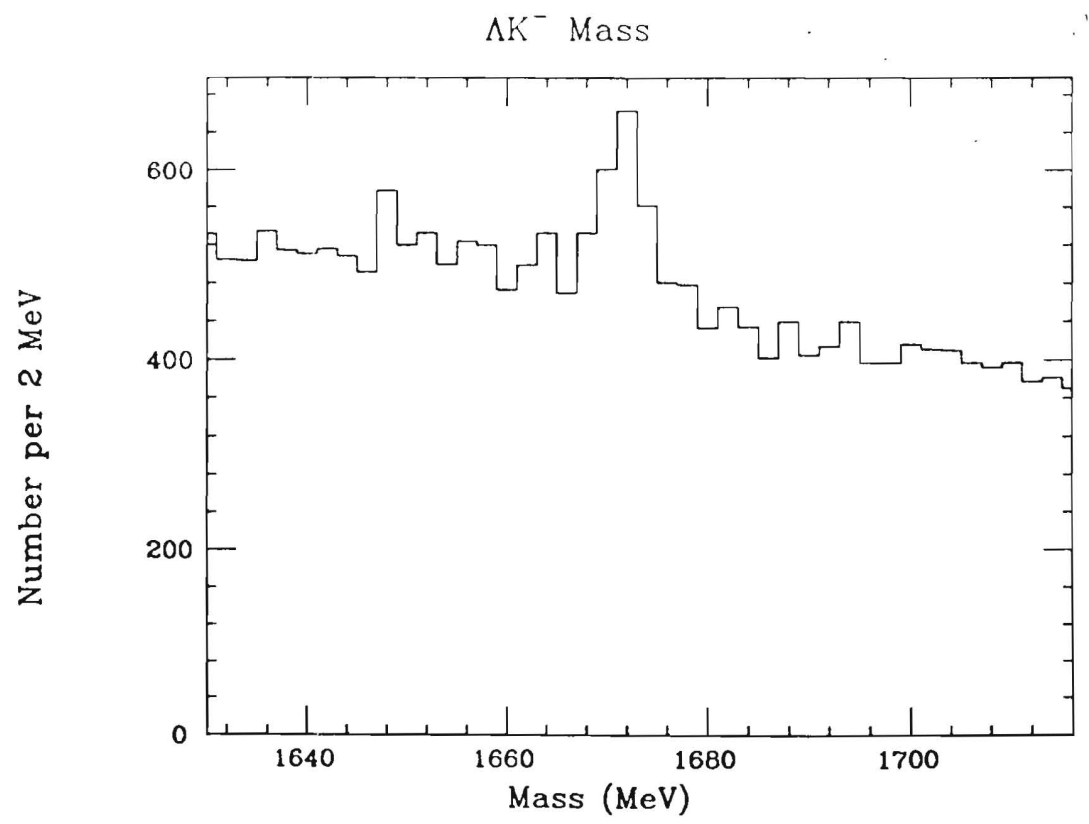

Figure 4.5: $\Lambda-\mathrm{K}^{-}$invariant mass from "good" 3-track events. This small sample of the data comes from 1 run. No cuts have been applied to separate $\Omega^{-} s$ from $\Xi^{-} s$. 


\subsection{Monte Carlo}

A computer program model of the experiment called the "Monte Carlo" was written. The purpose of the Monte Carlo was to simulate the experiment as closely as possible. It was used in the design of the experiment to study the effects on the acceptance of possible changes in the apparatus. It also was used to study the resolution of the detectors, the efficiency of the reconstruction program and possible backgrounds to the $\Omega^{-}$event sample.

The Monte Carlo program created $\Omega^{-}$at the target with an initial position and momentum. They were required to pass through the curved channel and collimator. The $\Omega^{-}$decayed according to an exponential decay distribution of the known lifetime into the chosen daughter particles. The $\Omega^{-}$could be given an initial vector or tensor polarization, if desired, in any direction. The daughters were allowed to decay, if their lifetimes were less than $1 \times 10^{-8}$ seconds. All decays were required to conserve energy and momentum and occur with the proper decay parameters.

The detectable decay products were traced through the apertures of the apparatus. Appropriate multiple coulomb scattering with Moliere tails deflected the charged particles at each piece of material in the spectrometer. The charged particles were bent at the BM109s with the measured transverse momentum. Small non-uniformities in the magnetic field of the BM109s were determined to have a negligible effect on the particle trajectories. If the event satisfied the trigger requirements, the positions of the charged particles at each MWPC and SSD were digitized in a manner identical to the data. Care was taken to insure that each detector was given the correct resolution and detection efficiency. For example, if a charged-particle passed through the region near the center of two wires in a MWPC, both wires were struck. The size of the overlapping region and the detector efficiencies were determined from analysis of the single track and three track data.

The hits were then reconstructed with the same reconstruction program used on the data. Monte Carlo parameters such as the initial momentum spectrum, and beam spot 
size were fine-tuned so that reconstructed Monte Carlo events mocked the data closely. Tapout files identical to those made for the data were an optional product of the Monte Carlo. These were used to study the performance of the event selection cuts and the polarization anaylsis.

It is important that the characteristics of the Monte Carlo model closely match the characteristics of the data. Figures 4.6 - 4.13 show a variety of Monte Carlo vs. Data comparisons. The comparisons include the position of the $\Omega^{-} \mathrm{s}$ at the exit of the collimator, the transverse momentum at the downstream target, the momentum of the $\Omega^{-}$and all the decay products, the $\Lambda$ and $\Omega^{-}$mass plots and vertices, the geometric $\chi^{2}$, the decay products position in the trigger chambers $\mathrm{C} 8$ and $\mathrm{C} 9$, the angular distributions of the $\Lambda$ in the $\Omega^{-}$rest frame and the angular distributions of the proton in the $\Lambda$ rest frame. Naturally, the cuts described in the next section used in selecting $\Omega^{-}$events from the data have been applied to the data and Monte Carlo events shown here. 

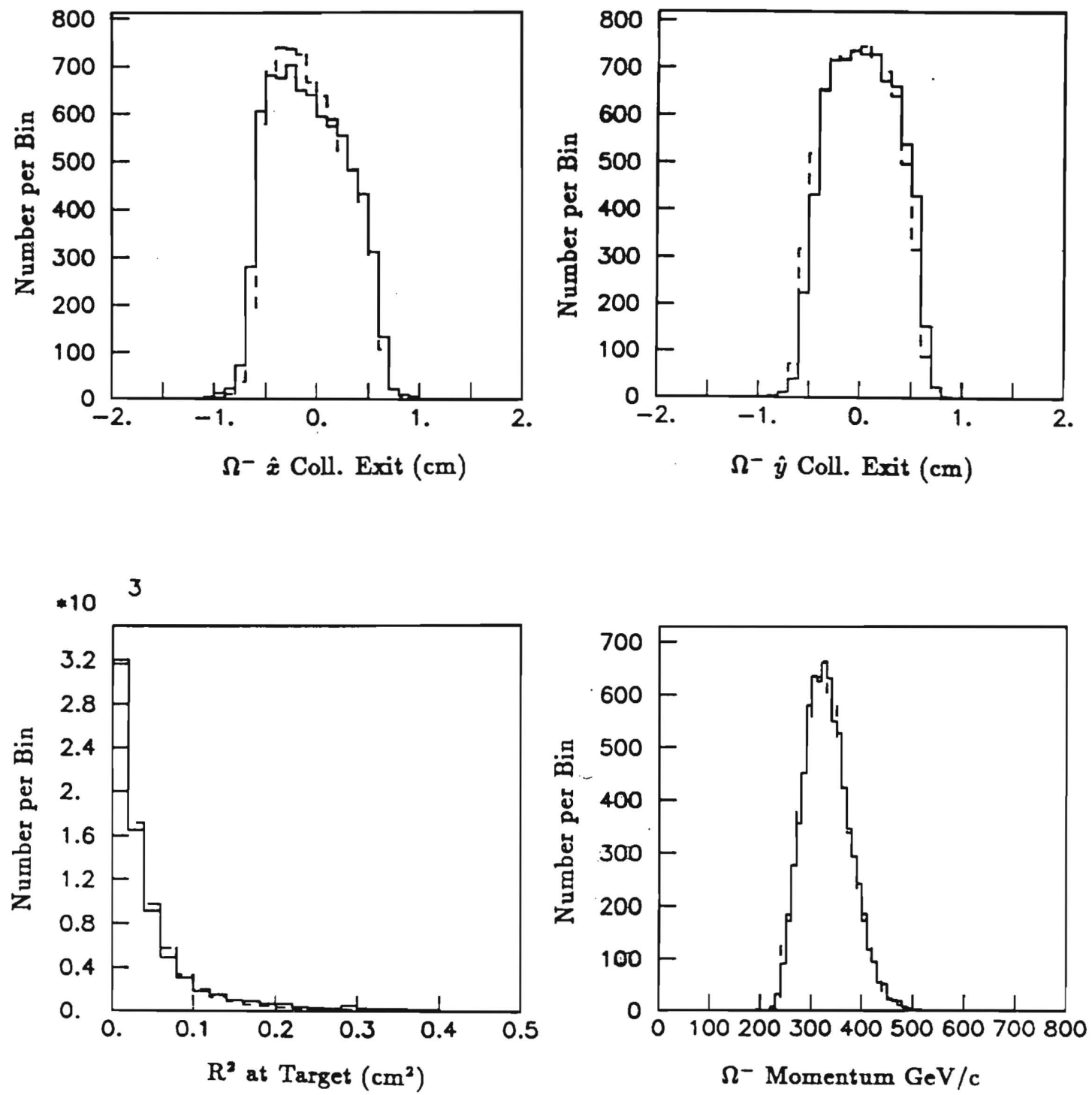

Figure 4.6: $\Omega^{-}$Monte Carlo vs Data. Shown are the $\Omega^{-}$position at the exit of the charged particle collimator, the radial distance-squared from the target center, and the $\Omega^{-}$momentum. Solid lines represent data. Dashed lines represent the Monte Carlo. (-450 Amp $0 \mathrm{mR}$ Phase 2). 

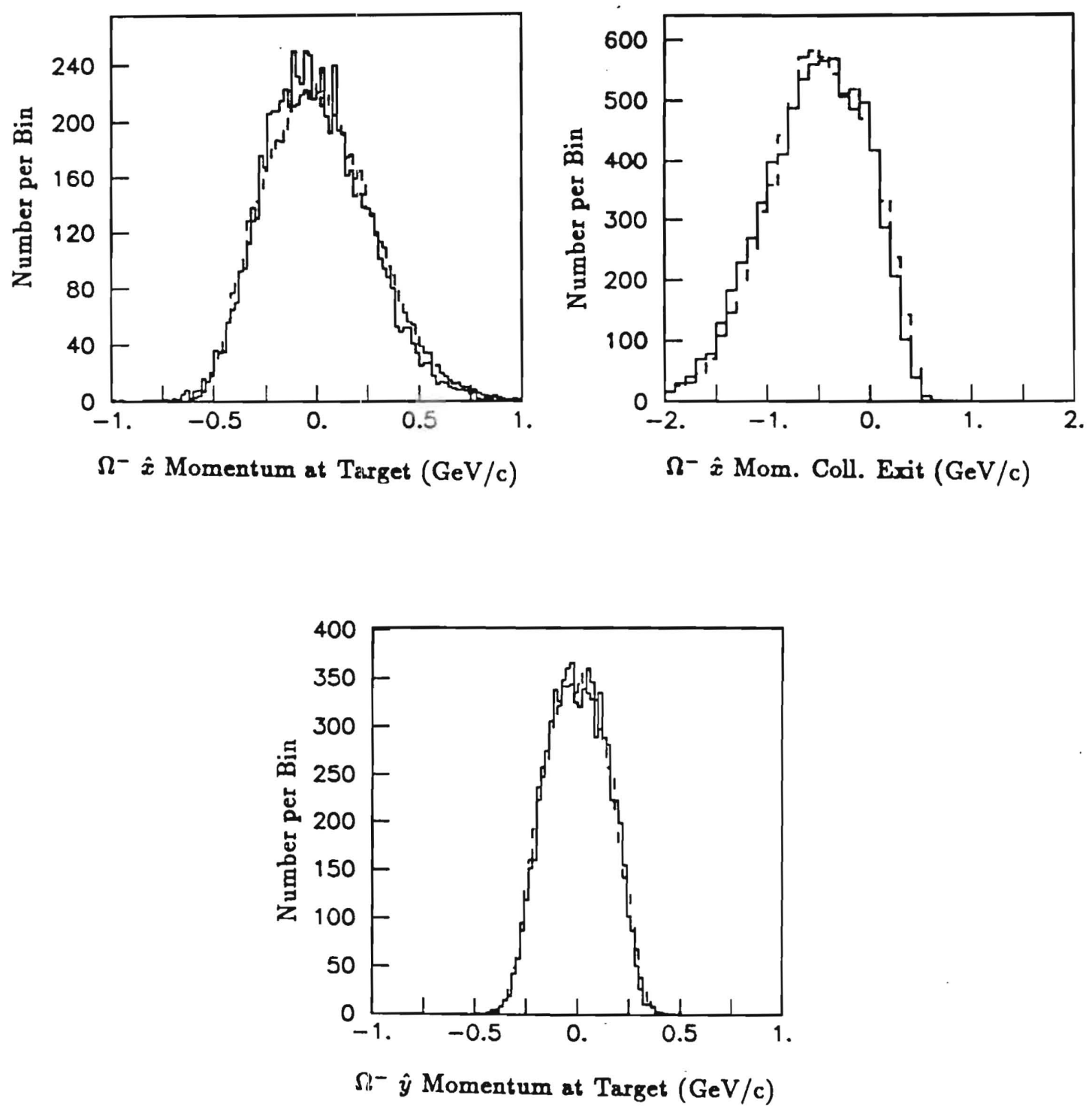

Figure 4.7: $\Omega^{-}$Monte Carlo vs Data. The $\Omega^{-}$transverse momentum components at the target and at the collimator exit. Solid lines represent data. Dashed lines represent the Monte Carlo. (-450 Amp $0 \mathrm{mR}$ Phase 2). 

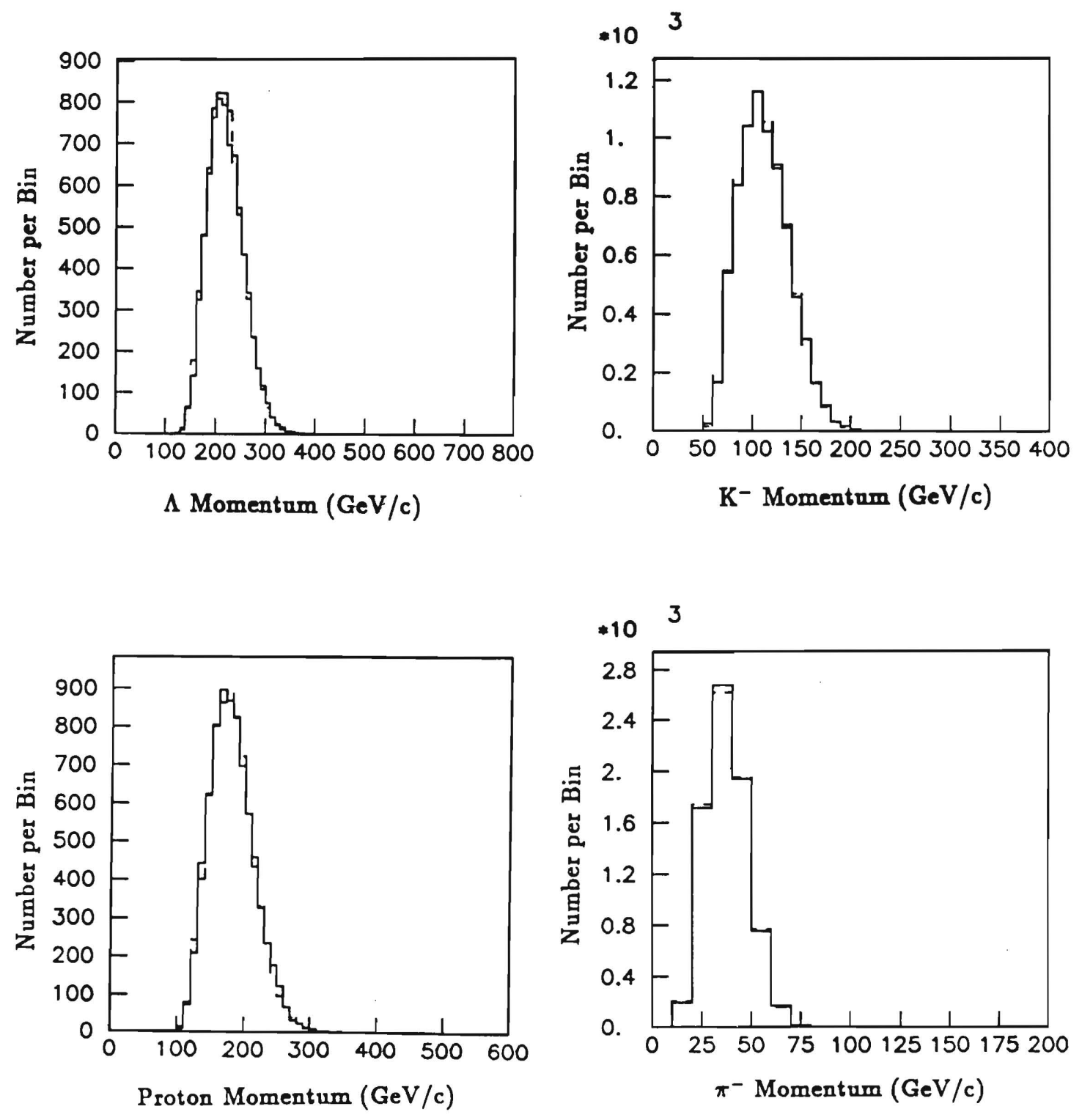

Figure 4.8: $\Omega^{-}$Monte Carlo vs Data. The momentum of the decay products. Solid lines represent data. Dashed lines represent the Monte Carlo. (-450 Amp $0 \mathrm{mR}$ Phase 2). 

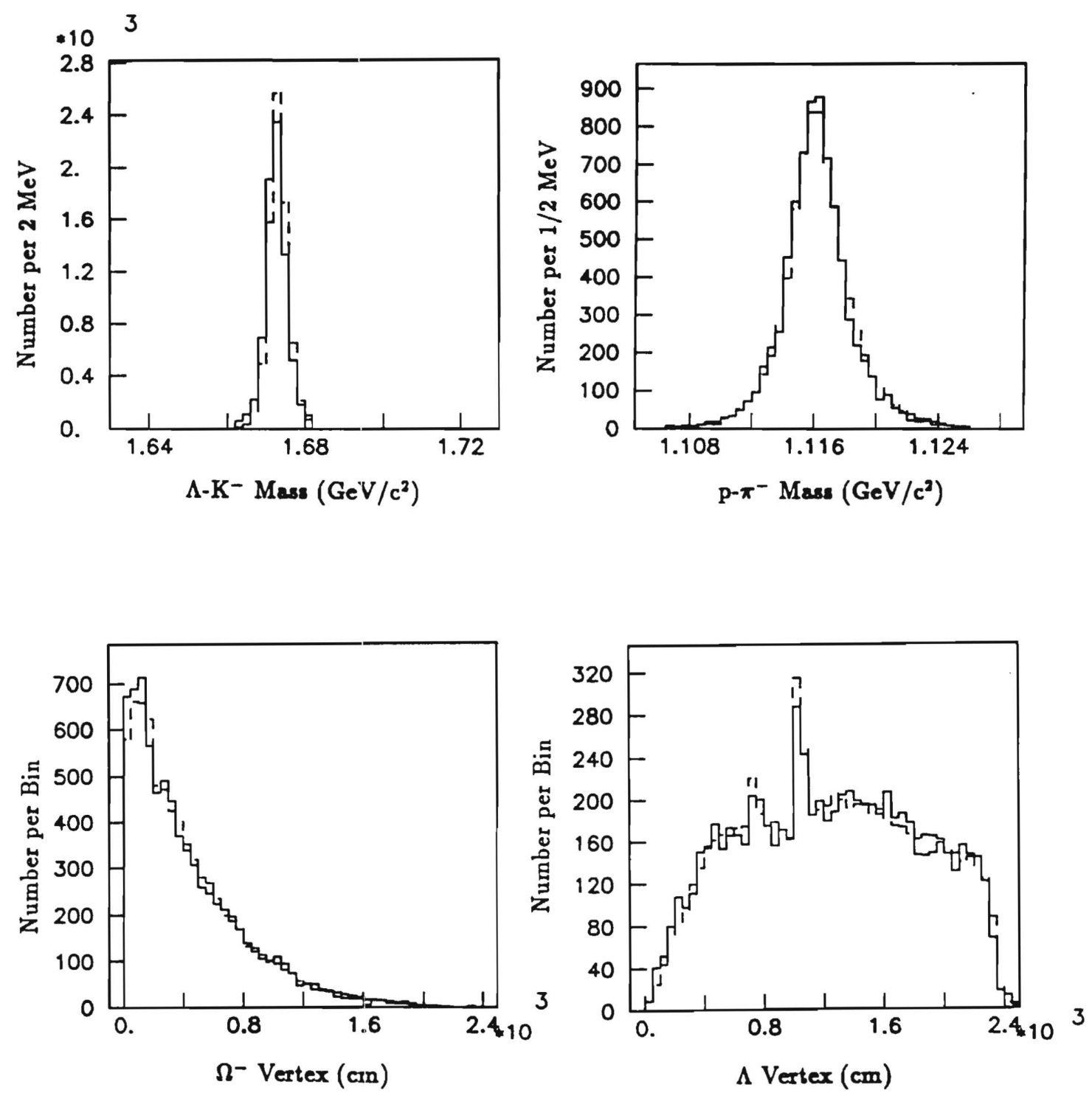

Figure 4.9: $\Omega^{-}$Monte Carlo vs Data. The $\Lambda-\mathrm{K}$ and $\mathrm{p}-\pi$ invariant masses and decay vertices. Solid lines represent data. Dashed lines represent the Monte Carlo. (-450 Amp $0 \mathrm{mR}$ Phase 2). 

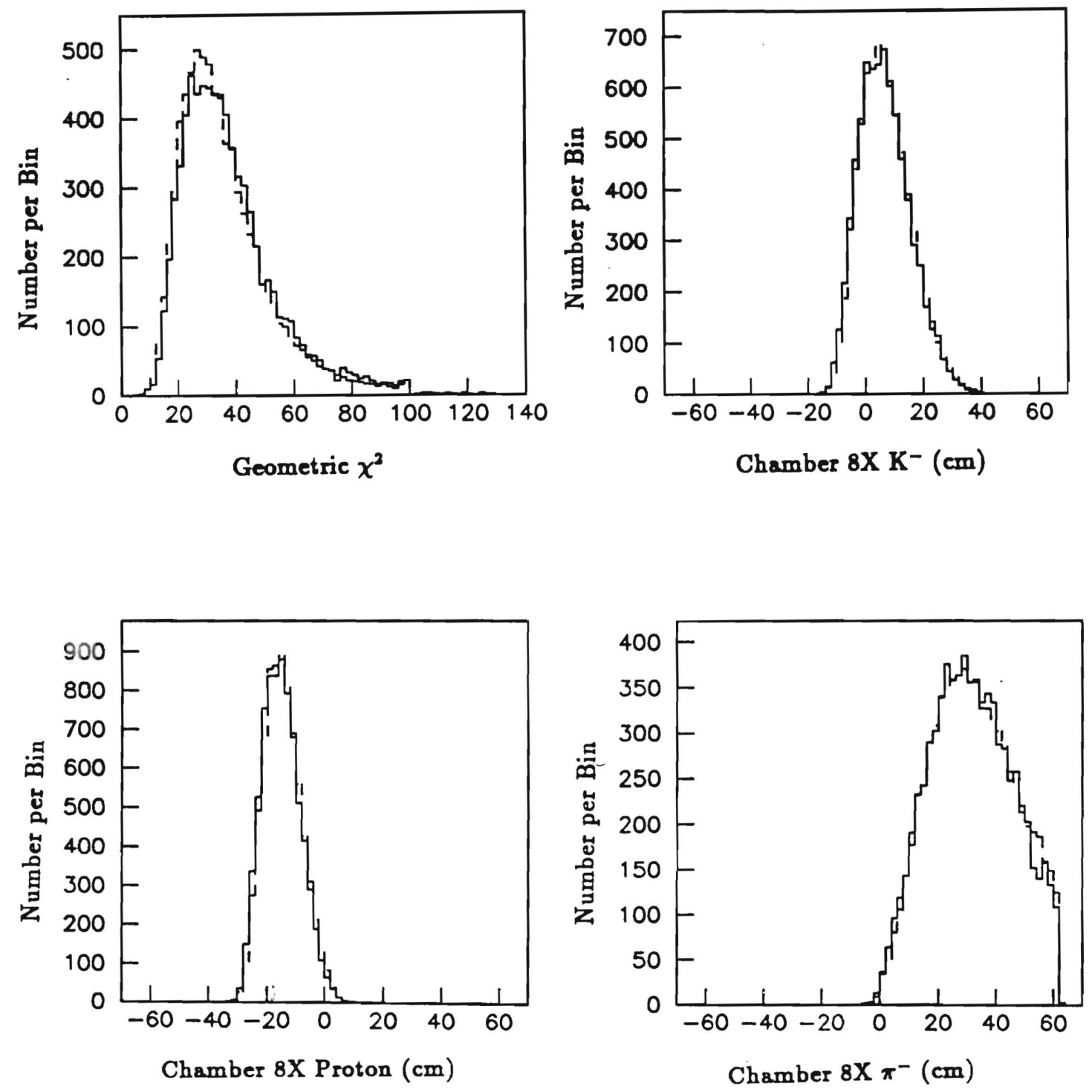

Figure 4.10: $\Omega^{-}$Monte Carlo vs Data. The geometric $\chi^{2}$ and the daughter distributions in MWPC C8. The trigger bounday is close to $x=0$. Solid lines represent data. Dashed lines represent the Monte Carlo. (-450 Amp $0 \mathrm{mR}$ Phase 2). 

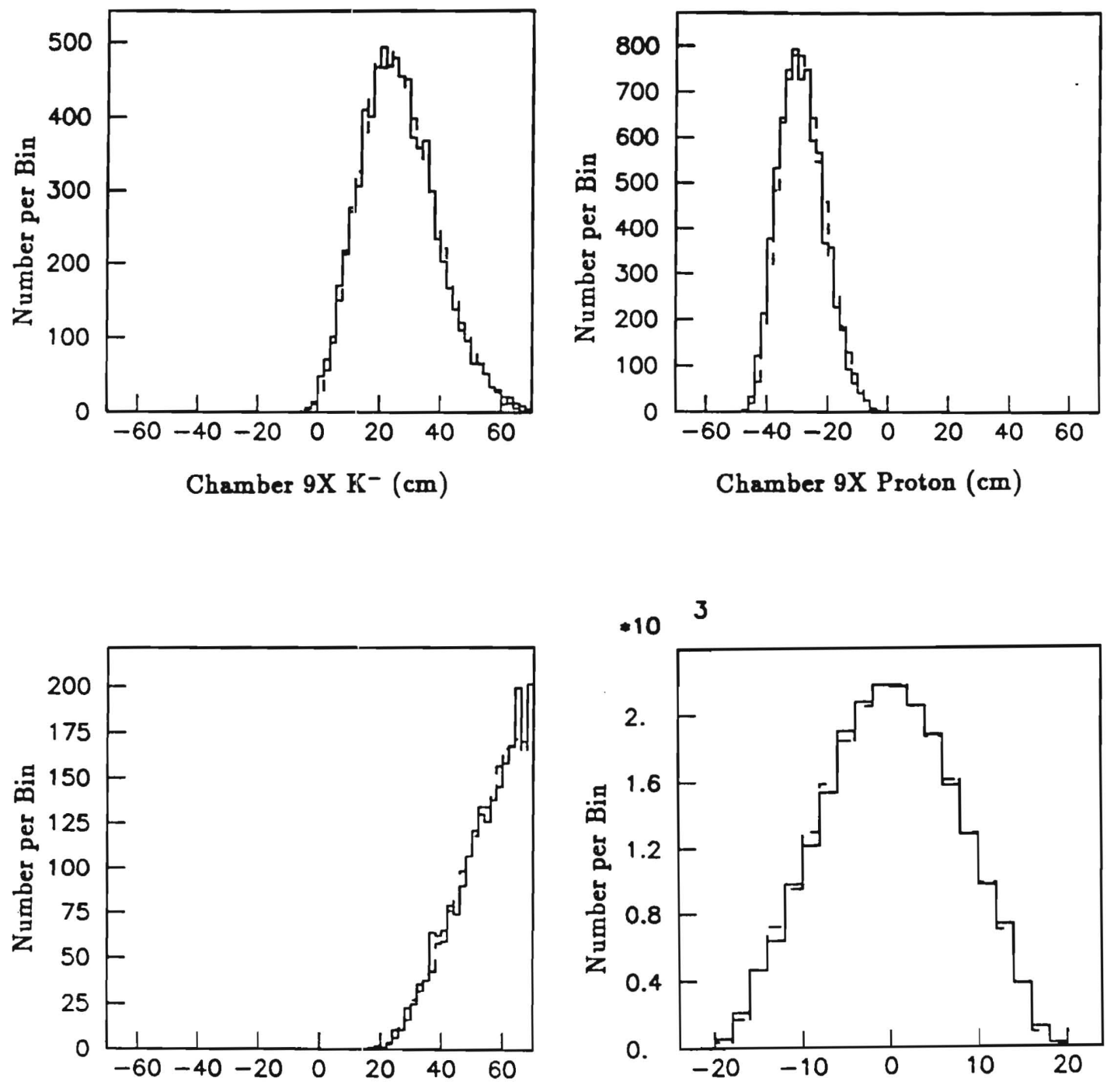

Chamber $9 \times \pi^{-}(\mathrm{cm})$

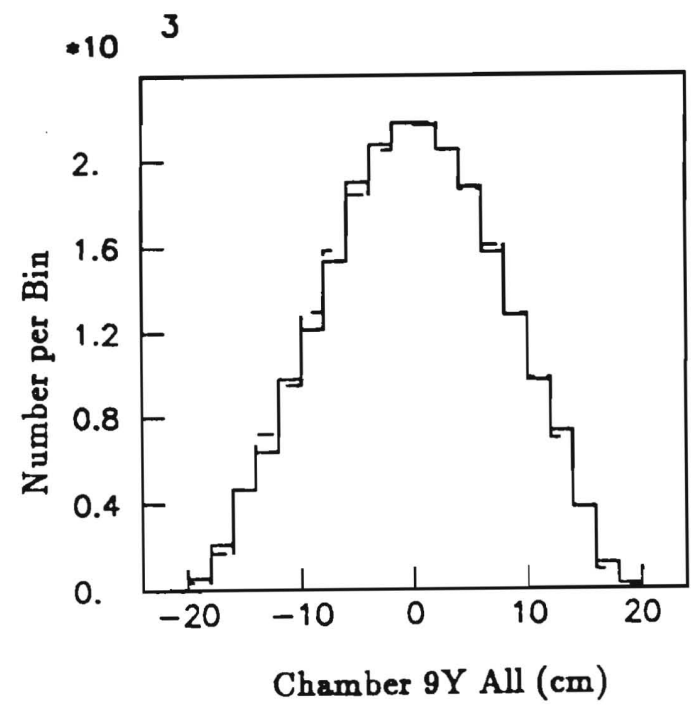

Figure 4.11: $\Omega^{-}$Monte Carjo vs Data. The daughter distributions in MWPC C9. The trigger boundary is close to $\mathrm{x}=0 \mathrm{~cm}$. Solid lines represent data. Dashed lines represent the Monte Carlo. (-450 Amp $0 \mathrm{mR}$ Phase 2). 

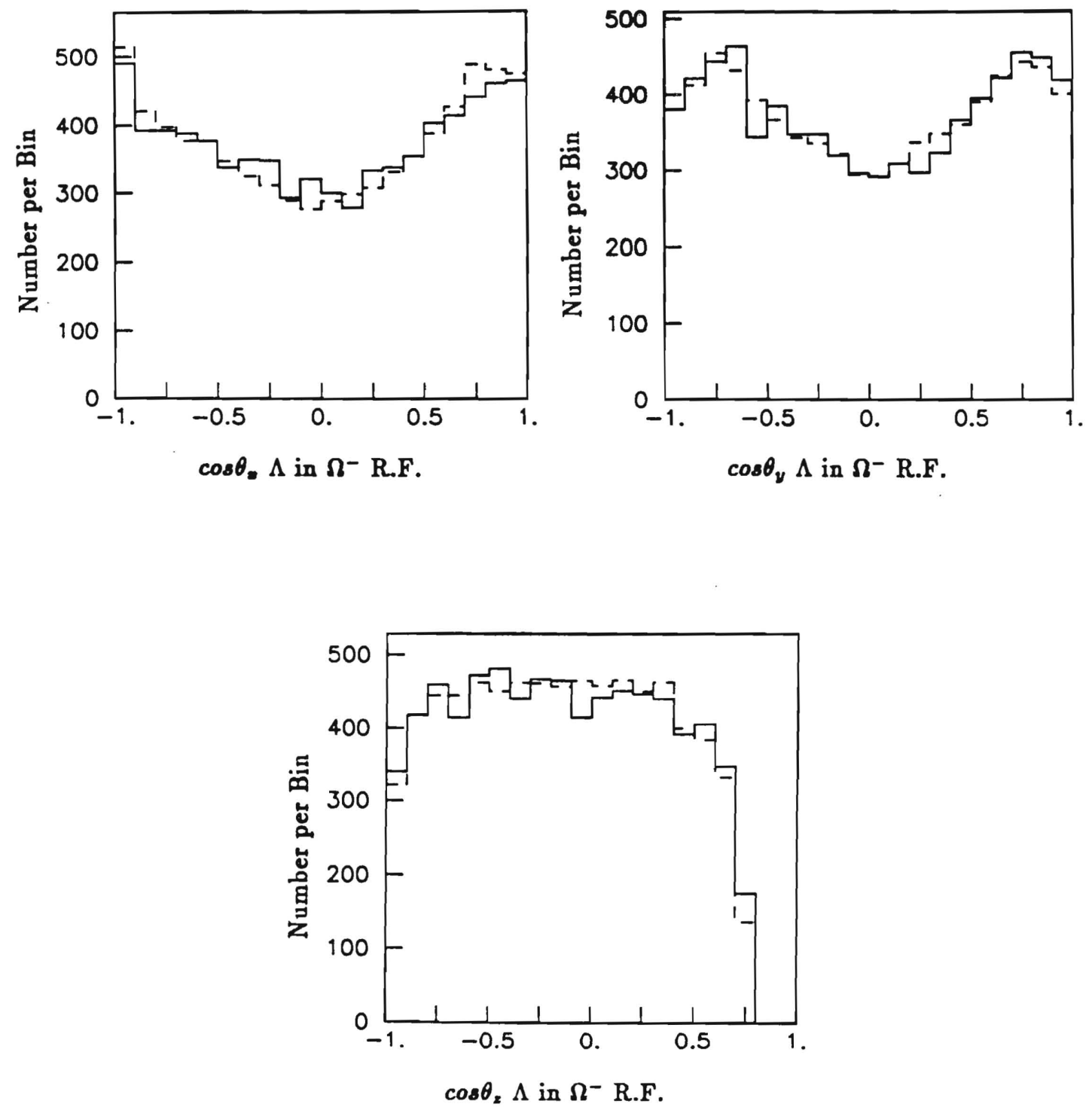

Figure 4.12: $\Omega^{-}$Monte Carlo vs Data. Shown are angular distributions of the $\Lambda$ in the $\Omega^{-}$rest frame. Solid lines represent data. Dashed lines represent the Monte Carlo. (-450 Amp $0 \mathrm{mR}$ Phase 2). 

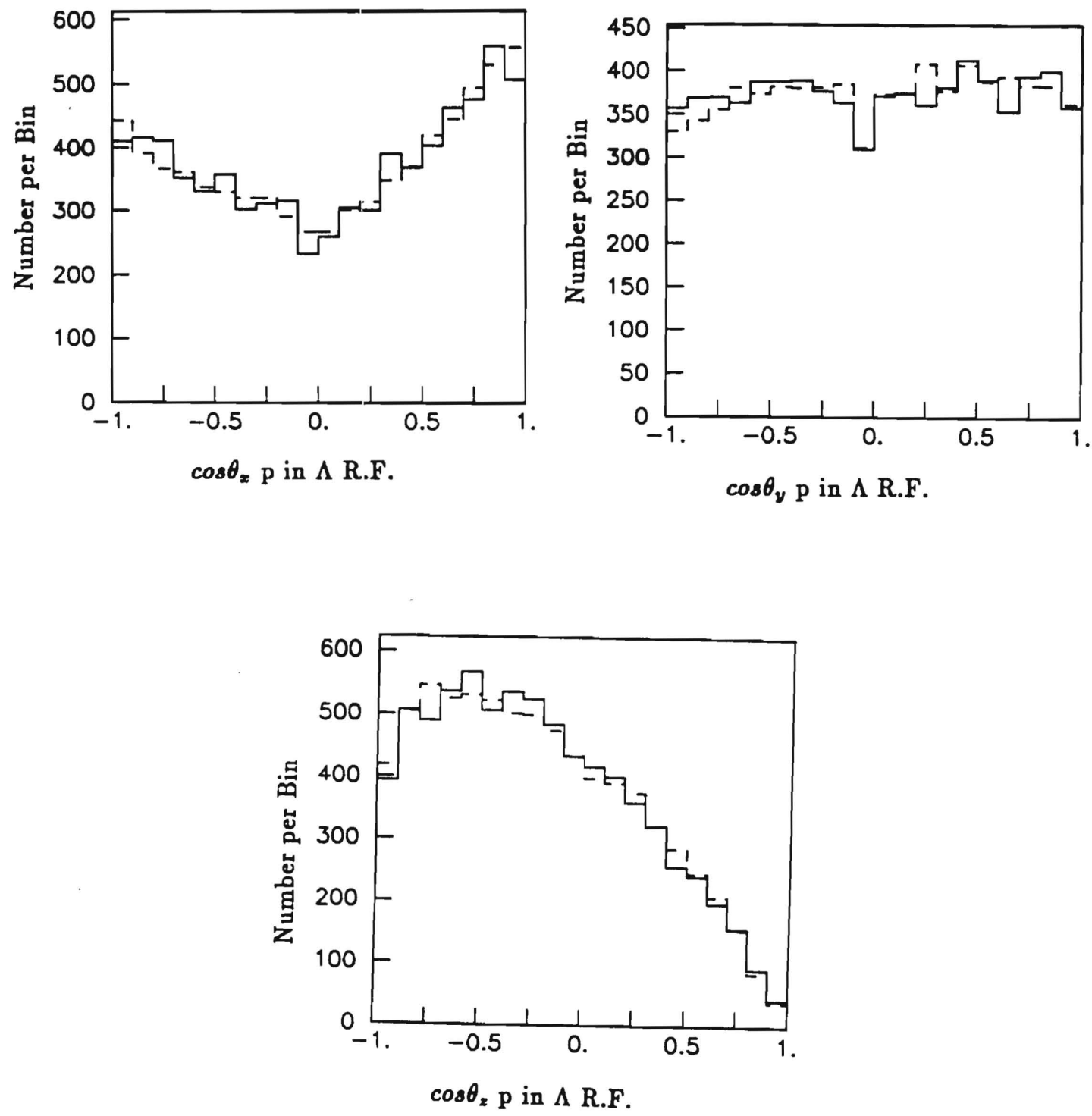

Figure 4.13: $\Omega^{-}$Monte Carlo vs Data. Shown are the angular distributions of the proton in the $\Lambda$ rest frame. Solid lines represent data. Dashed lines represent the Monte Carlo. (-450 Amp $0 \mathrm{mR}$ Phase 2). 


\subsection{Omega Minus Event Selection and Background}

The $\Omega^{-}$signal of Figure 4.5 is embedded in a large background which has a variety of origins including poorly reconstructed events and misidentified decays. The largest potential background comes from $\Xi^{-} \rightarrow \Lambda \pi^{-}$decays. The production cross-section ratio of $\Xi^{-} \mathrm{s}$ to $\Omega^{-} \mathrm{s}$ is roughly 75 . Other potential backgrounds include $\Omega^{-} \rightarrow \Xi^{0} \pi^{-}$, $\Omega^{-} \rightarrow \Xi^{-} \pi^{0}$ and $\mathrm{K}^{-} \rightarrow \pi^{+} \pi^{-} \pi^{-}$decays. The production cross-section of $\mathrm{K}^{-}$to $\Xi^{-}$is roughly 10 . This section contains an explanation for each of the cuts used to separate good $\Omega^{-}$events from this background. Table 4.2 relates each cut to a "cut number". Table 4.3 shows the number of $\Omega^{-}$candidates remaining in the data and an estimate of the number of background events remaining at each cut as determined with the Monte Carlo.

Poorly reconstructed events were eliminated first. The nominal value of the $\Lambda$ mass is $1.1156 \mathrm{GeV} / \mathrm{c}^{2}$. The reconstructed $\mathrm{p}-\pi^{-}$invariant mass was required to fall between 1.106 and $1.126 \mathrm{GeV} / \mathrm{c}^{2}$. This cut events on the tails of the $\Lambda$ mass plot (Figure 4.3). Events with a geometric $\chi^{2}$ greater than 100 were eliminated.

Next, the $\Omega^{-}$s were separated from the $\Xi^{-}$s. Events for which the $\Lambda^{-} \pi^{-}$mass fell between 1.297 and $1.345 \mathrm{GeV} / \mathrm{c}^{2}$ were eliminated at this stage (see Figure 4.4). A similar, but looser, cut had already been made by the reconstruction program in selecting events for tapout tapes. The reason for the cut is that an event which had a $\Lambda-\pi^{-}$mass close to $1321.4 \mathrm{GeV} / \mathrm{c}^{2}$ was more likely a $\Xi^{-}$rather than an $\Omega^{-}$regardless of the $\Lambda-\mathrm{K}^{-}$mass.

At this stage, events where the $\Lambda$ vertex was located upstream of the primary vertex or which had a primary vertex located near the collimator were rejected. The track finder already required that $\Lambda$ s decay downstream of their origin but the kinematic fitter had changed the $\Lambda$ vertex. Also, decays which occurred in the collimator were likely reconstructed poorly. A cut eliminated events with a primary vertex located in the Hyperon Magnet.

Next, a cut which eliminated background events was available because $\Lambda$ s from 


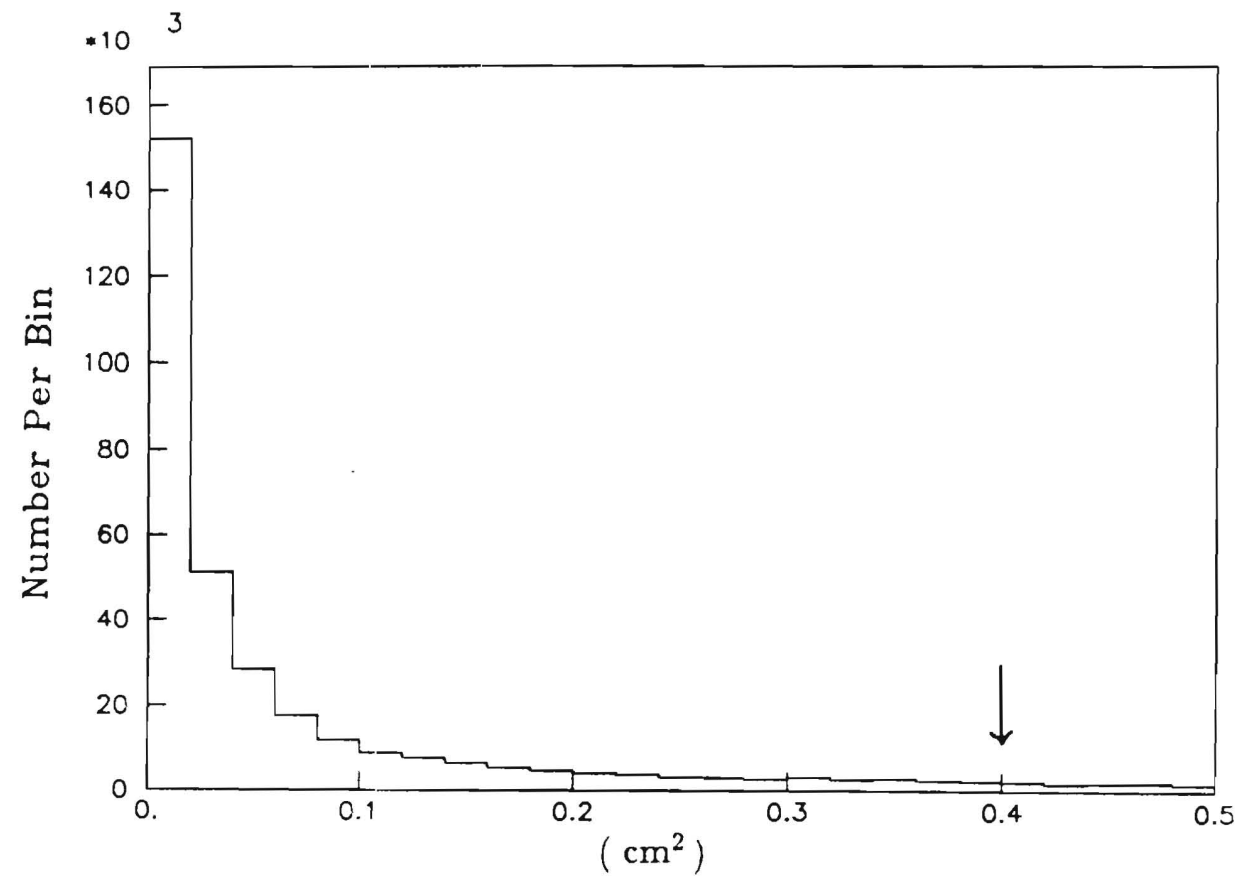

Figure 4.14: $R^{2}$ for events which satisfied all preceeding cuts. The cut was applied at the arrow.

$\Omega^{-} \rightarrow \Lambda \mathrm{K}^{-}$events which were reconstructed as $\Xi^{-} \rightarrow \Lambda \pi^{-}$events fell into one $\cos \theta$ region in the $\Xi^{-}$rest frame. The projection of the $\Lambda$ direction on the $\hat{z}$ axis of the spectrometer coordinates in the $\Xi^{-}$rest frame was $\cos \theta_{\Lambda}=\hat{\Lambda} \cdot \hat{z}$. The event was presumed a poorly reconstructed $\Xi^{-}$and rejected if $\cos \theta_{\Lambda}$ was greater than -0.40 .

The momentum vector of the primary particles was then projected back to the $x-y$ plane containing the midpoint of the production target. Events which pointed outside of a circle centered at the target-center with a radius-squared of $0.40 \mathrm{~cm}^{2}$ were rejected. These were poorly reconstructed events, background events or $\Omega^{-}$s which originated in the collimator. Figure 4.14 shows the target pointing distribution for events which passed all cuts up to the target pointing cut.

Then, the $\mathrm{K}^{-}$angular distribution was calculated in the $\Omega^{-}$rest frame with respect to axes parallel to the spectrometer coordinate system. The distribution of $\cos \theta_{\mathrm{K}^{-}}=$ $\hat{\mathrm{K}}^{-} \cdot \hat{z}$ versus $\phi_{\mathrm{K}^{-}}=\tan ^{-1}\left(\hat{\mathrm{K}}^{-} \cdot \hat{y} / \hat{\mathrm{K}}^{-} \cdot \hat{x}\right)$ for all of the events up until this cut is shown in Figure 4.15. For all $\phi_{\mathrm{K}^{-}}$, the region between $-1 .<\cos \theta_{\mathrm{K}^{-}}<-0.75$ is contaminated by $\Xi^{-}$events reconstructed under the wrong $\left(\Omega^{-}\right)$hypothesis. Kinematic 
calculation and Monte Carlo studies demonstrated this. The depleted band in Figure 4.15 is a result of the $\Xi^{-}$mass cut. There is also an excess of events in the region $-0.75<\cos \theta_{\mathrm{K}^{-}}<-0.40$ with $\phi_{\mathrm{K}^{-}} \sim 0$. Monte Carlo studies indicated these were $\Xi^{-}$ events which decayed in the collimator. Figure 4.16 shows $\cos \theta_{\mathrm{K}^{-}}$versus $\phi_{\mathrm{K}^{-}}$for Monte Carlo $\Xi^{-}$events including collimator decays. Since the topologies of the $\Xi^{-} \rightarrow \Lambda \pi^{-}$ and $\Omega^{-} \rightarrow \Lambda \mathrm{K}^{-}$decays are identical, the spectrometer could not always distinguish $\Xi^{-}$events from $\Omega^{-}$events. Even if the spectrometer resolution was perfect, $\Xi^{-}$events would fall in the band in $\cos \theta_{\mathrm{K}}$ - from -0.8 to -1.0 . A cut was made which removed all of these $\Xi^{-}$. The event was cut if any of the following conditions was true:

$$
\begin{gathered}
\cos \theta_{\mathrm{K}^{-}}<-0.75 \\
-0.75<\cos \theta_{\mathrm{K}^{-}}<-0.65 \text { and }-60<\phi_{\mathrm{K}^{-}}<60, \\
-0.65<\cos \theta_{\mathrm{K}^{-}}<-0.55 \text { and }-36<\phi_{\mathrm{K}^{-}}<36, \\
-0.55<\cos \theta_{\mathrm{K}^{-}}<-0.45 \text { and }-24<\phi_{\mathrm{K}^{-}}<24, \\
-0.45<\cos \theta_{\mathrm{K}^{-}}<-0.40 \text { and }-12<\phi_{\mathrm{K}^{-}}<12,
\end{gathered}
$$

where $\cos \theta_{\mathrm{K}^{-}}$is given in radians and $\phi_{\mathrm{K}^{-}}$is given in degrees. This was done at the cost of a region of the kaon phase space, as shown in Figure 4.15.

Finally, the $\Lambda-\mathrm{K}$ invariant mass was required to fall between 1.662 and $1.682 \mathrm{GeV} / \mathrm{c}^{2}$. 


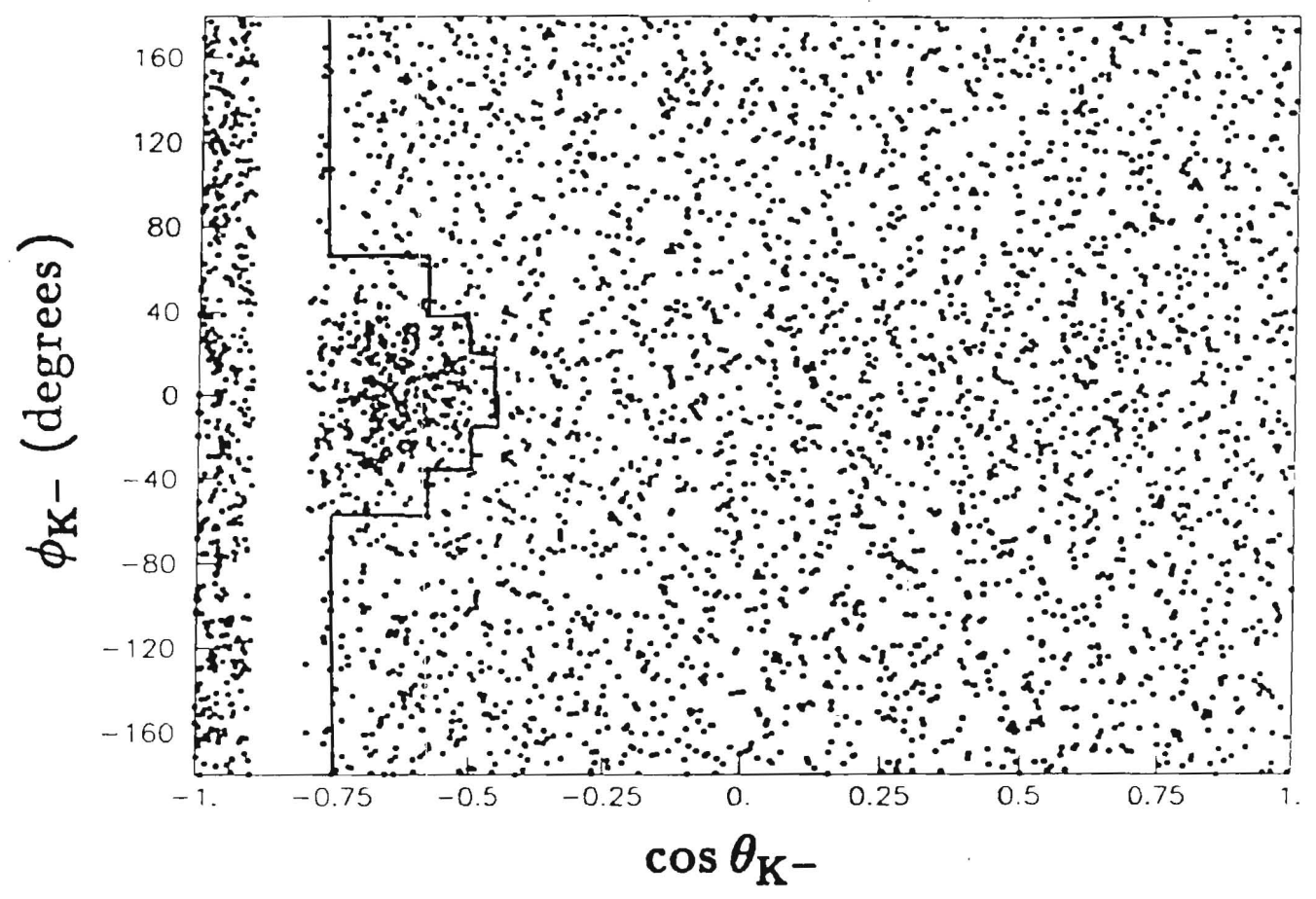

Figure 4.15: $\cos \theta_{K^{-}}$versus $\phi_{K^{-}}$for all events preceeding the outlined cut. The cut-out band at -0.8 was a result of the $\Xi^{-}$mass cut.

\begin{tabular}{||c|c||}
\hline $\begin{array}{c}\text { Cut } \\
\text { Number }\end{array}$ & Description \\
\hline 0 & IOK=0 \\
\hline 1 & $1.63<m_{\Lambda K}<1.73 \mathrm{GeV} / \mathrm{c}^{2}$ \\
& NOT $\left(1.308<m_{\Lambda \pi}<1.340 \mathrm{GeV} / \mathrm{c}^{2}\right)$ \\
\hline 2 & $1.106<m_{p \pi}<1.126 \mathrm{GeV} / \mathrm{c}^{2}$ \\
\hline 3 & NOT $\left(1.297<m_{\Lambda \pi}<1.345 \mathrm{GeV} / \mathrm{c}^{2}\right)$ \\
\hline 4 & Decay verter $\Lambda>\Omega^{-}>0 \mathrm{~cm}$ \\
\hline 5 & $\Lambda$ Angular Dist. in $\Xi^{-}$r.f. \\
\hline 6 & $\mathrm{R}^{2}<0.40 \mathrm{~cm}^{2}$ \\
\hline 7 & $\mathrm{~K}^{-}$Angular Dist. in $\Omega^{-}$r.f. \\
\hline 8 & $1.662<m_{\Lambda K}<1.682 \mathrm{GeV} / \mathrm{c}^{2}$ \\
\hline
\end{tabular}

Table 4.2: Summary of the cuts used to select $\Omega^{-} \mathbf{s}$. A cut number is assigned to each cut for use in Table 4.3. Cuts 0 and 1 were made while processing the raw data tapes as described in Section 4.1. The remainder were applied to the "tapout" tapes. 


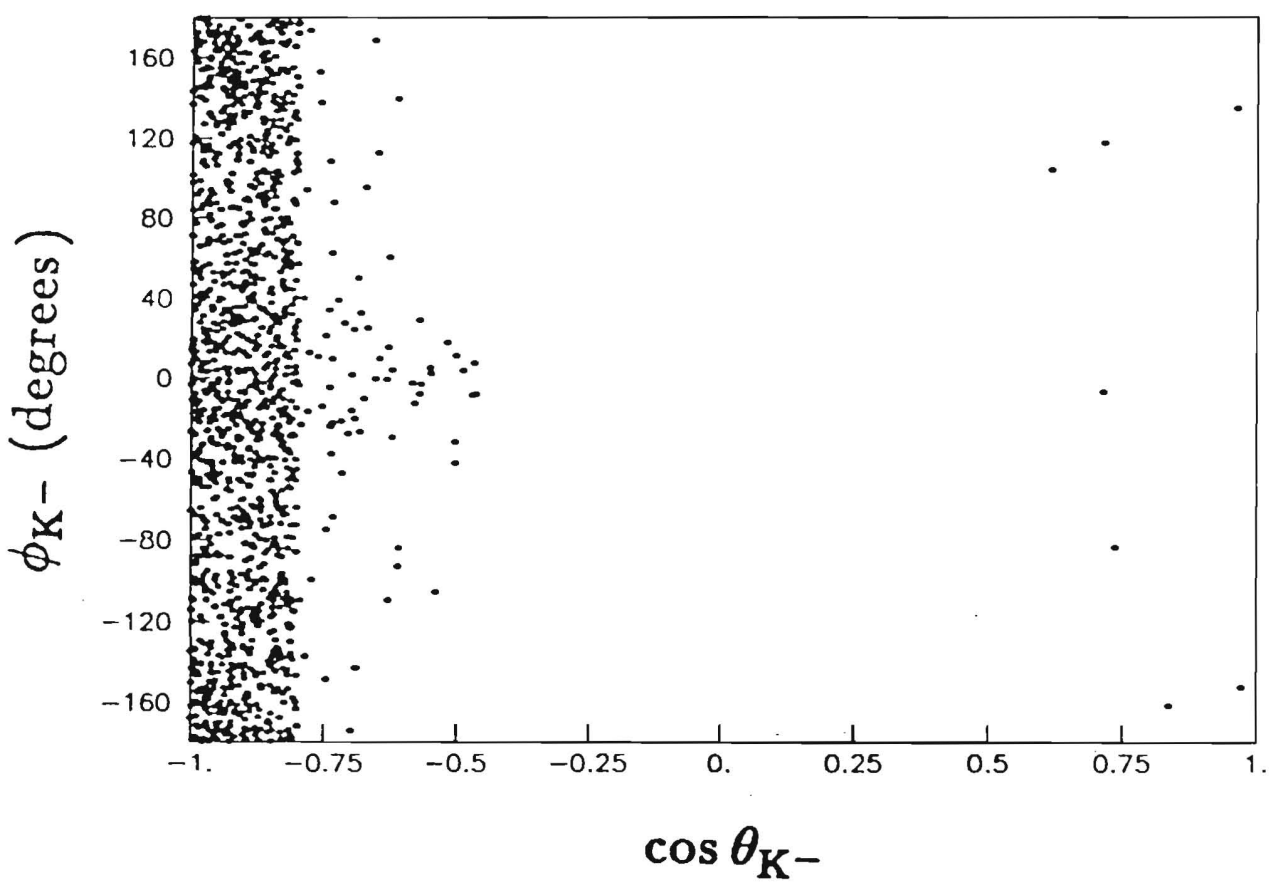

Figure 4.16: $\cos \theta_{K^{-}}$versus $\phi_{K^{-}}$for Monte Carlo $\Xi^{-}$events. The excess of events centered around $\phi_{\mathrm{K}^{-}}=0$ is from $\Xi^{-}$decays which occurred in the collimator. 


\begin{tabular}{|c|c|c|c|c|}
\hline $\begin{array}{c}\text { Cut } \\
\text { Number }\end{array}$ & $\begin{array}{c}\text { Data } \\
\text { Events } \\
\text { Remaining }\end{array}$ & $\begin{array}{c}\text { Scaled } \\
\Omega^{-} \rightarrow \Lambda \mathrm{K}^{-} \\
\text {Monte Carlo } \\
\text { Events } \\
\text { Remaining }\end{array}$ & $\begin{array}{c}\text { Scaled } \\
\Xi^{-} \rightarrow \Lambda \pi^{-} \\
\text {Monte Carlo } \\
\text { Events } \\
\text { Remaining }\end{array}$ & $\begin{array}{c}\text { Scaled } \\
\Omega^{-} \rightarrow \Xi^{0} \pi^{-} \\
\text {Monte Carlo } \\
\text { Events } \\
\text { Remaining }\end{array}$ \\
\hline 0 & $19.7 \mathrm{M}$ & 265130 & $19.3 \mathrm{M}$ & 70660 \\
\hline 1 & 1640551 & 232550 & $464 \mathrm{~K}$ & 49390 \\
\hline 2 & 963486 & 229120 & $378 \mathrm{~K}$ & 49050 \\
\hline 3 & 633286 & 220200 & $296 \mathrm{~K}$ & 48710 \\
\hline 4 & 409567 & 179040 & 35500 & 38070 \\
\hline 5 & 349452 & 178700 & 31400 & 33960 \\
\hline 6 & 240990 & 169440 & 3220 & 13380 \\
\hline 7 & 185045 & 158120 & $320 \pm 200$ & 13380 \\
\hline 8 & 160518 & 156745 & $0_{-0}^{+122}$ & 3773 \\
\hline
\end{tabular}

Table 4.3: Summary of the cuts used to select $\Omega^{-}$s. The first column is the data. The next 3 columns are Monte Carlo events scaled so that the bottom and top lines equal the number of data events. The Monte Carlo events were generated with the Hyperon Magnet at -450 amps. 
There were 160518 events which passed all of the cuts. Less than less than $2.5 \%$ of these events were background. The main background after cuts was the $\Omega^{-} \rightarrow \Xi^{0} \pi^{-}$. Table 4.3 shows that there are an estimated 3773 poorly reconstructed $\Omega^{-} \rightarrow \Xi^{0} \pi^{-}$and $0^{+122}{ }_{0} \Xi^{-} \rightarrow \Lambda \pi^{-}$which fake $\Omega^{-} \rightarrow \Lambda \mathrm{K}^{-}$. The $\Omega^{-} \rightarrow \Xi^{-} \pi^{0}$ and $\mathrm{K}^{-} \rightarrow 3 \pi$ decays were shown by the Monte Carlo to be negligible. The number of $\Omega^{-} \mathrm{s}$ for each of the major data-taking conditions are shown in Tables 4.4 to Table 4.6. Figures 4.17 to 4.25 show the final $\Omega^{-}$vertex distribution, the $\Lambda$ vertex distribution, the geometric $\chi^{2}$, the $\pi^{-}$, proton $\mathrm{K}^{-}$, and $\Omega^{-}$momentum distributions, the proton-pion invariant mass and the $\Lambda$-K invariant mass for the $\Omega^{-}$event sample which passed all of the cuts. 


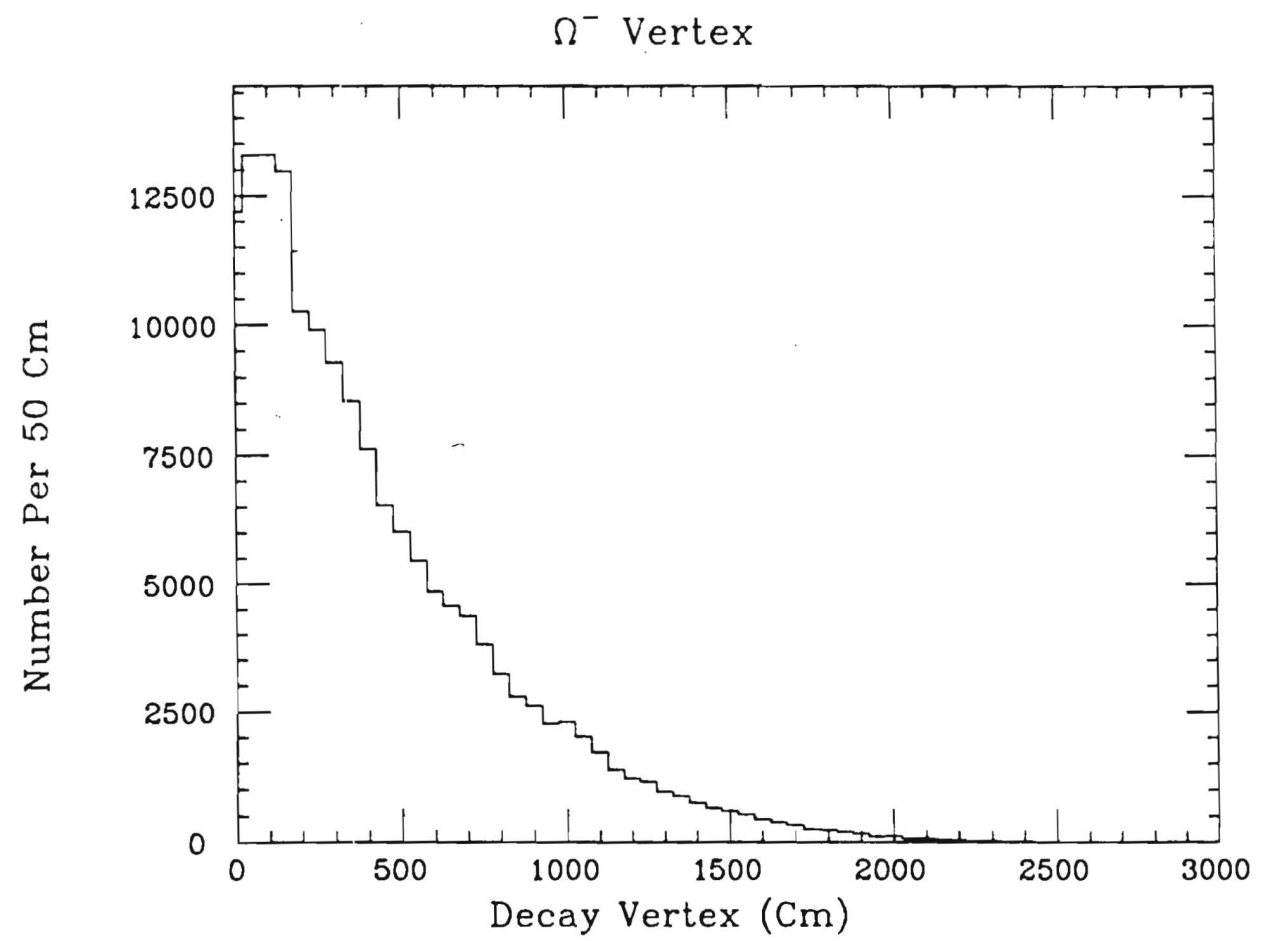

Figure 4.17: $\Omega^{-}$vertex distribution of the full sample. 


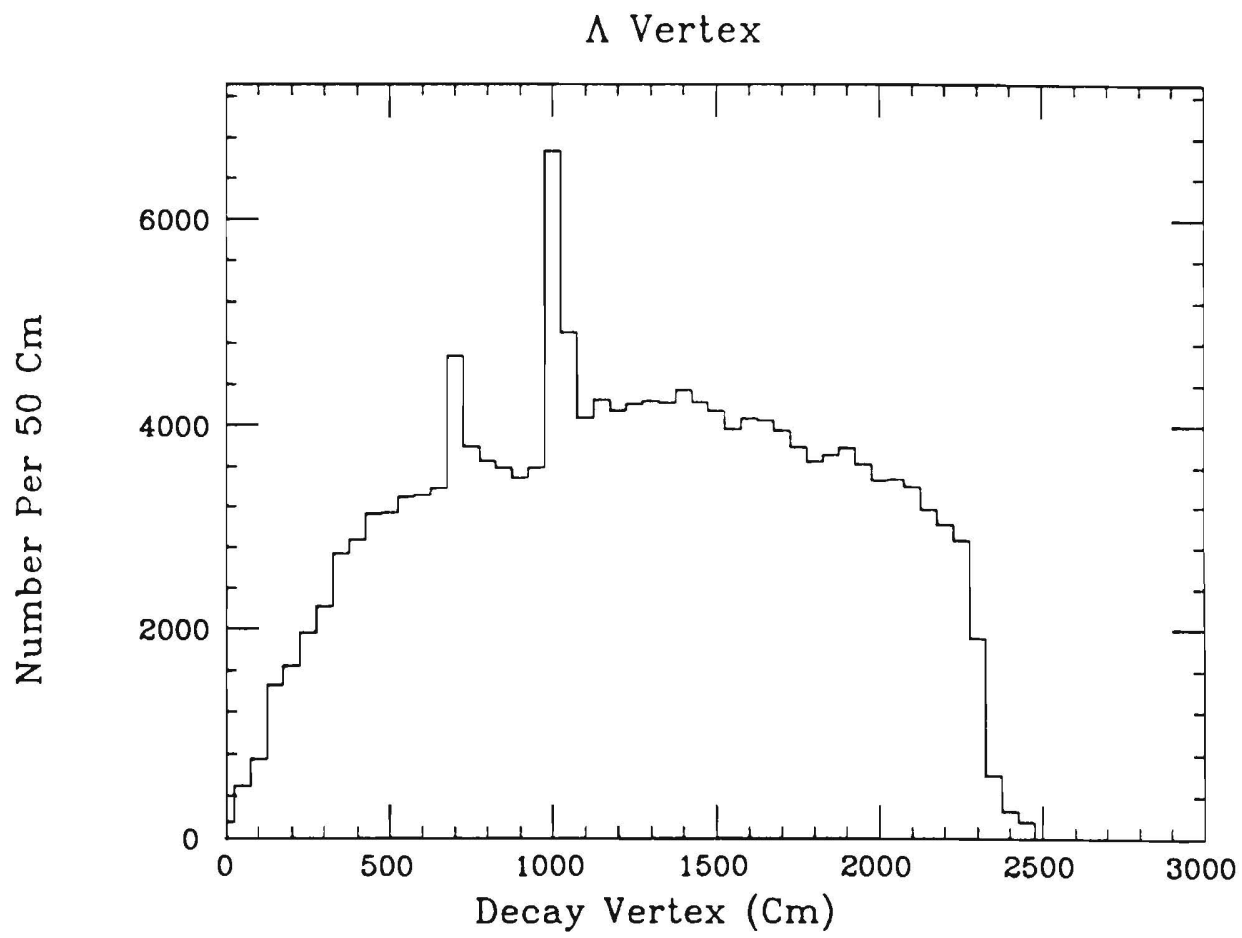

Figure 4.18: $\Lambda$ vertex distribution for all $\Omega^{-}$s. The reconstruction program is responsible for the peaks in the distribution at C2 and C3. It tends to move vertices just upstream of the chamber to the $z$ location of the chamber. The effect is reproduced in the Monte Carlo events. 


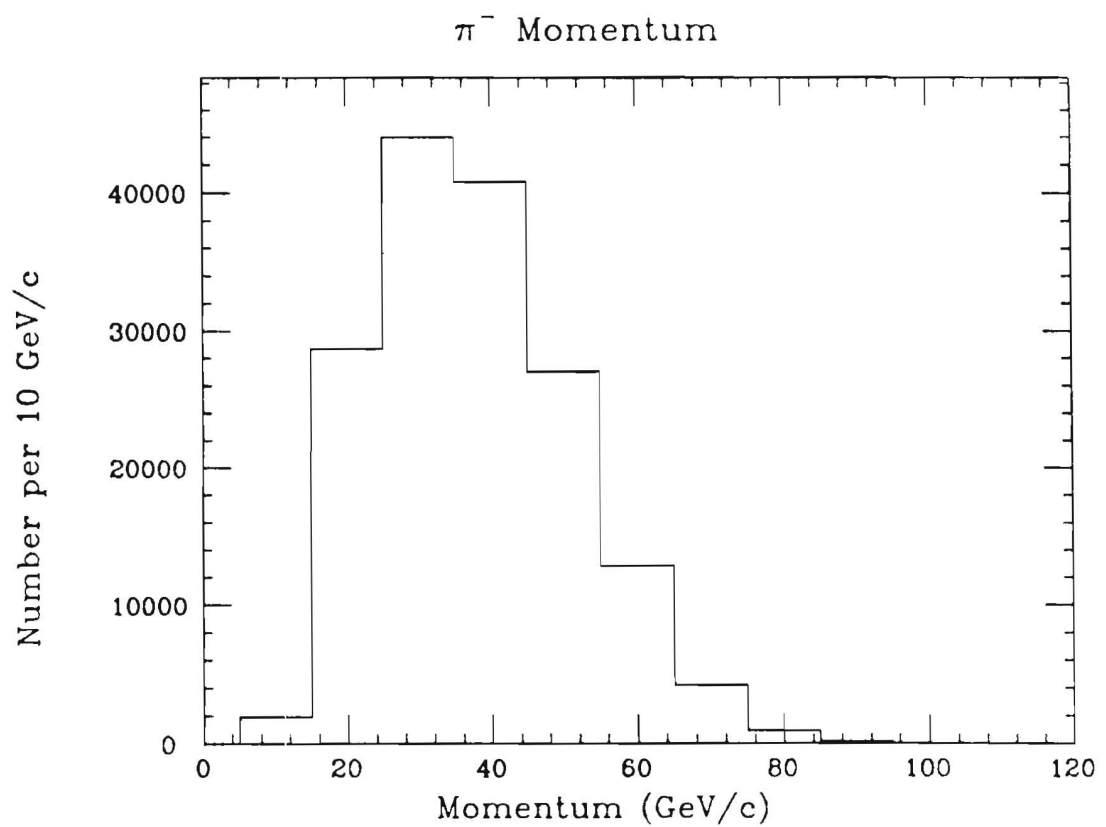

Figure 4.19: $\pi^{-}$momentum distribution for all $\Omega^{-} \mathrm{s}$.

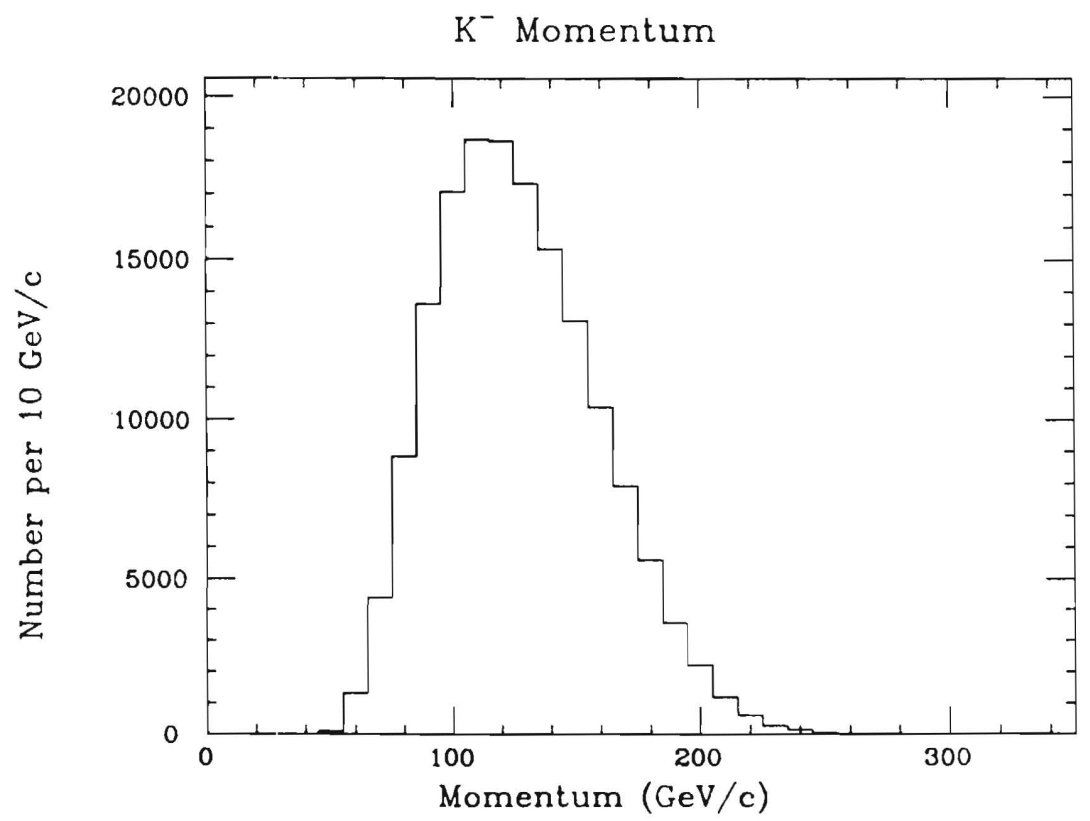

Figure 4.20: $\mathrm{K}^{-}$momentum distribution for all $\Omega^{-} \mathrm{s}$. 


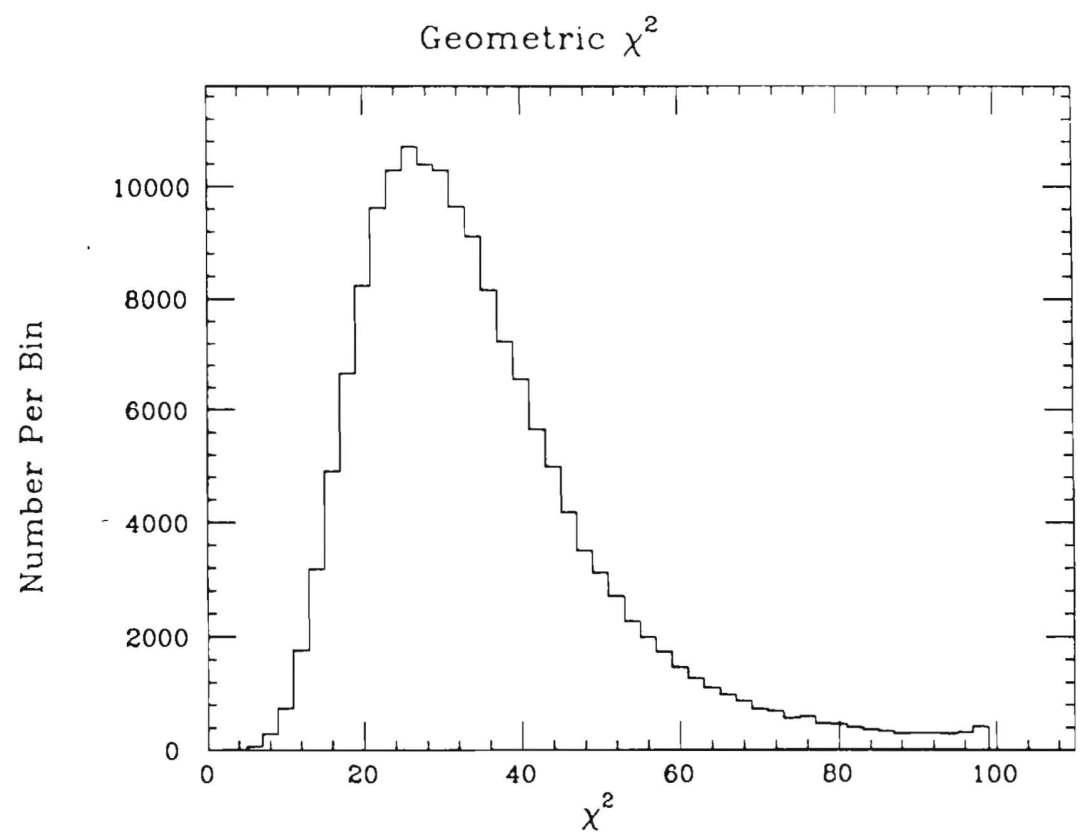

Figure 4.21: Geometric $\chi^{2}$ for all $\Omega^{-}$events.

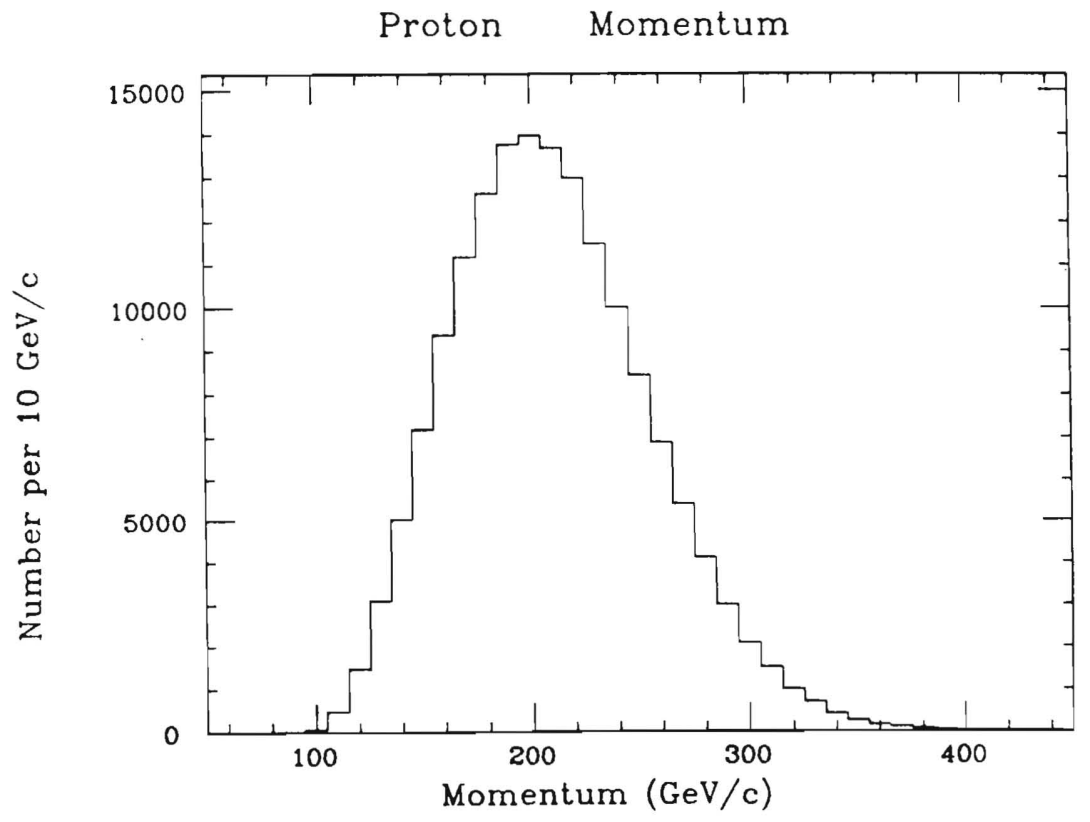

Figure 4.22: Proton momentum distribution from all $\Omega^{-}$-events. 


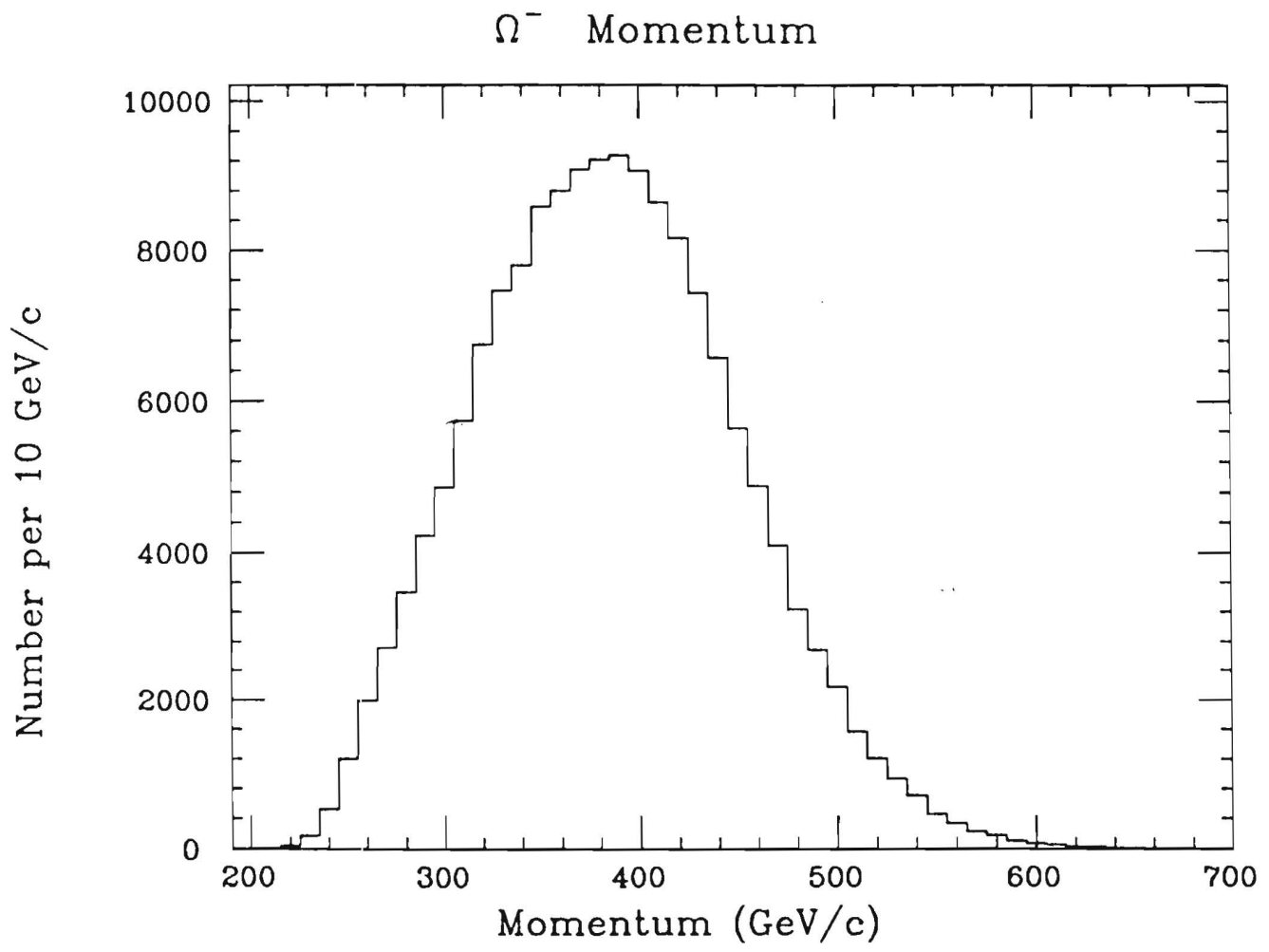

Figure 4.23: $\Omega^{-}$momentum distribution from all $\Omega^{-}$events. 


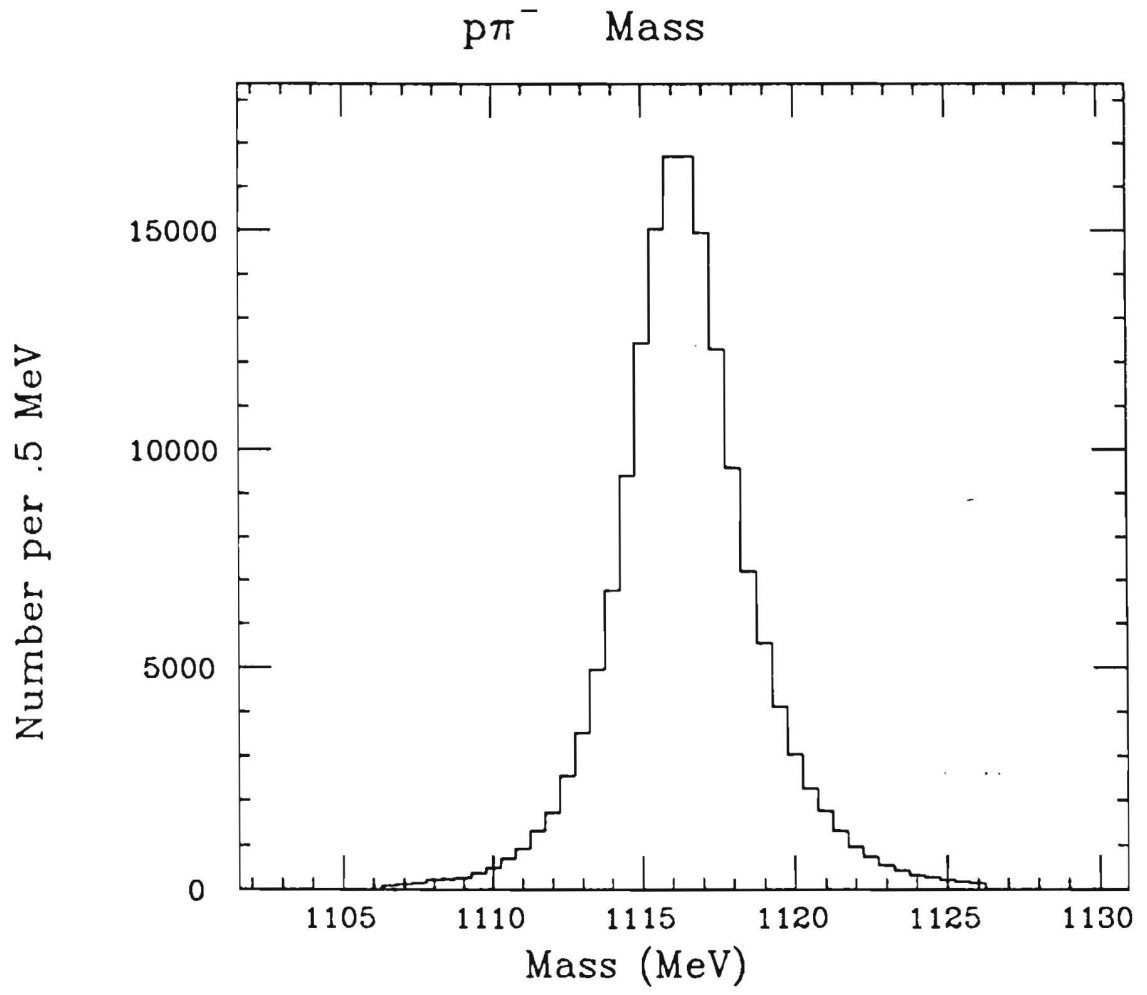

Figure 4.24: $\mathrm{p}-\pi^{-}$invariant mass distribution of all $\Omega^{-}$events. 

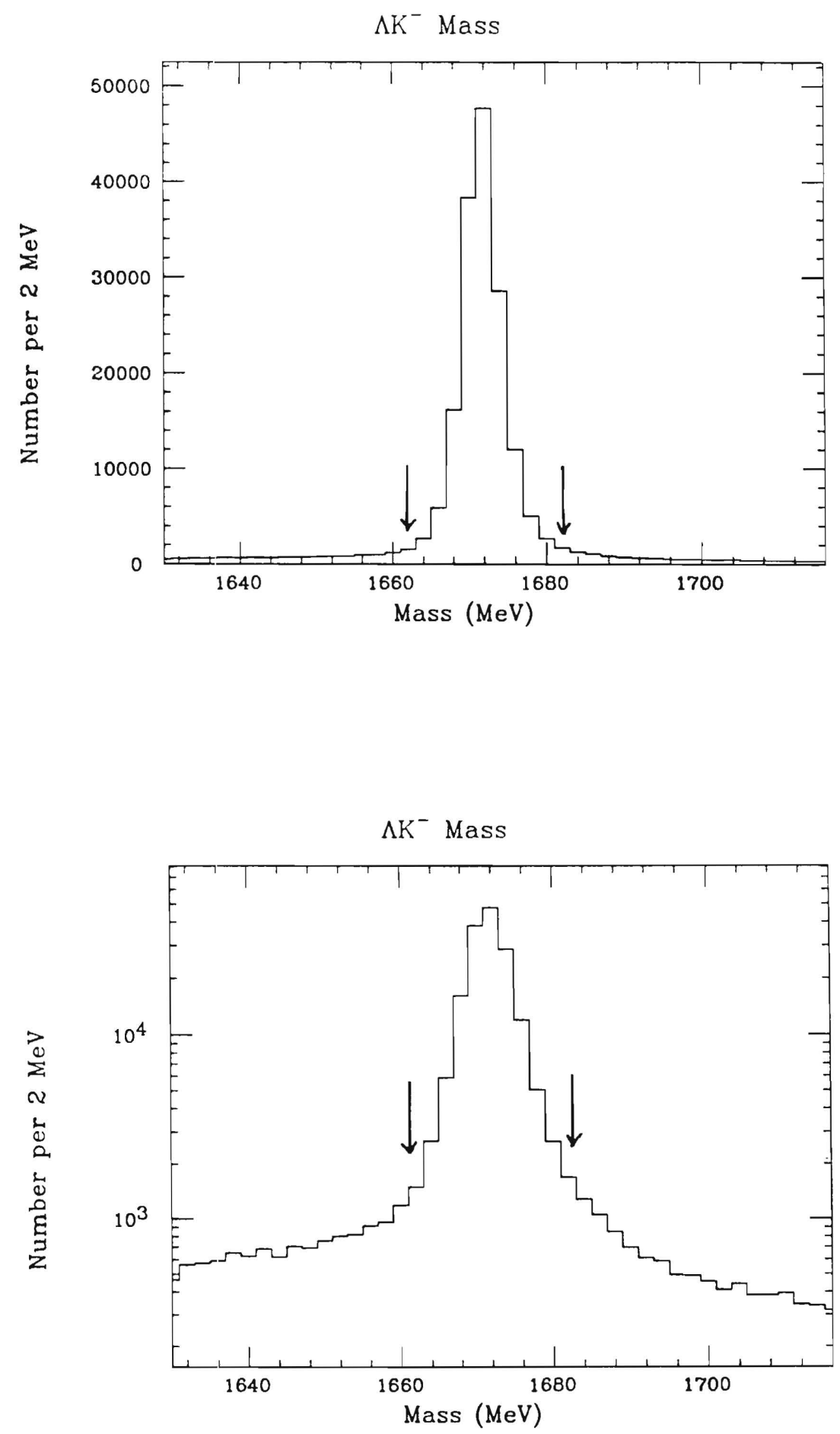

Figure 4.25: Lambda - $\mathrm{K}^{--}$invariant mass distribution for all $\Omega^{-} \mathrm{s}$. These are two plots of the same data. The bottom plot has a log vertical scale. The position of the mass cut is shown by the arrows. 


\begin{tabular}{|c|c|c|c|c|}
\hline $\begin{array}{l}\text { Hyperon } \\
\text { Magnet } \\
\text { (Amps) }\end{array}$ & $\begin{array}{r}\text { BM109s } \\
\text { (Amps) }\end{array}$ & $\begin{array}{c}\text { Vertical } \\
\text { Targeting } \\
\text { Angles }(\mathrm{mR})\end{array}$ & $\begin{array}{c}\Omega^{-} \\
\text {Candidates } \\
\text { Tapped Out }\end{array}$ & $\begin{array}{c}\Omega^{-} \\
\text {Passed } \\
\text { All Cuts }\end{array}$ \\
\hline \multirow[t]{4}{*}{-500} & \multirow[t]{2}{*}{-2500} & -2.5 & 40652 & 4381 \\
\hline & & +2.5 & 38504 & 4304 \\
\hline & \multirow[t]{2}{*}{2500} & -2.5 & 44555 & 3839 \\
\hline & & +2.5 & 57125 & 4915 \\
\hline \multirow[t]{4}{*}{-1000} & \multirow[t]{2}{*}{-2500} & -2.5 & 144038 & 16205 \\
\hline & & +2.5 & 145652 & 15943 \\
\hline & \multirow[t]{2}{*}{2500} & -2.5 & 99950 & 9664 \\
\hline & & +2.5 & 88494 & 8493 \\
\hline \multirow[t]{6}{*}{-1700} & \multirow[t]{3}{*}{-2500} & -2.5 & 87389 & 9323 \\
\hline & & +2.5 & 72476 & 7291 \\
\hline & & +0.0 & 12064 & 634 \\
\hline & \multirow[t]{3}{*}{2500} & -2.5 & 53057 & 5159 \\
\hline & & +2.5 & 51872 & 4794 \\
\hline & & +0.0 & 12677 & 645 \\
\hline \multirow[t]{6}{*}{-2500} & \multirow[t]{3}{*}{-2500} & -2.5 & 22440 & 2184 \\
\hline & & +2.5 & 20896 & 2013 \\
\hline & & +0.0 & 13109 & 610 \\
\hline & \multirow[t]{3}{*}{2500} & -2.5 & 16126 & 1480 \\
\hline & & +2.5 & 11757 & 1033 \\
\hline & & +0.0 & 12994 & 600 \\
\hline \multirow[t]{6}{*}{-3300} & \multirow[t]{3}{*}{-2500} & -2.5 & 41191 & 3647 \\
\hline & & +2.5 & 26287 & 2249 \\
\hline & & +0.0 & 13565 & 551 \\
\hline & \multirow[t]{3}{*}{2500} & -2.5 & 15525 & 1265 \\
\hline & & +2.5 & 14681 & 1203 \\
\hline & & +0.0 & 14276 & 596 \\
\hline
\end{tabular}

Table 4.4: Phase 1. Number of $\Omega^{-}$s for each beam condition. Main conditions. 


\begin{tabular}{|c|c|c|c|c|}
\hline $\begin{array}{l}\text { Hyperon } \\
\text { Magnet } \\
\text { (Amps) }\end{array}$ & $\begin{array}{c}\text { BM109s } \\
\text { (Amps) }\end{array}$ & $\begin{array}{c}\text { Horizontal } \\
\text { Targeting } \\
\text { Angles }(\mathrm{mR})\end{array}$ & $\begin{array}{c}\Omega^{-} \\
\text {Candidates } \\
\text { Tapped Out }\end{array}$ & $\begin{array}{c}\Omega^{-} \\
\text {Passed } \\
\text { All Cuts }\end{array}$ \\
\hline \multirow[t]{2}{*}{-1000} & -2500 & (Tgt Out) +0.0 & 5668 & 394 \\
\hline & 2500 & (Tgt Out) +0.0 & 5943 & 394 \\
\hline \multirow[t]{10}{*}{-1700} & -2500 & (Tgt Out) +0.0 & 6663 & 351 \\
\hline & 2500 & (Tgt Out) +0.0 & 7425 & 372 \\
\hline & -2500 & (Tgt Out) -1.3 & 5717 & 378 \\
\hline & \multirow[t]{2}{*}{2500} & (Tgt Out) -1.3 & 6942 & 423 \\
\hline & & (Tgt Out) +1.3 & 2576 & 142 \\
\hline & \multirow[t]{3}{*}{-2500} & (Tgt In) -1.3 & 5684 & 340 \\
\hline & & $($ Tgt In $)+1.3$ & 6056 & 359 \\
\hline & & (Tgt In) -2.2 & 3236 & 282 \\
\hline & \multirow[t]{2}{*}{2500} & (Tgt In) -1.3 & 6437 & 368 \\
\hline & & $(\mathrm{Tgt}$ In) +1.3 & 5185 & 280 \\
\hline \multirow[t]{10}{*}{-2500} & -2500 & (Tgt Out) +0.0 & 6883 & 323 \\
\hline & 2500 & (Tgt Out) +0.0 & 6922 & 348 \\
\hline & \multirow[t]{2}{*}{-2500} & (Tgt Out) -1.3 & 6610 & 414 \\
\hline & & (Tgt Out) +1.3 & 4813 & 252 \\
\hline & \multirow[t]{2}{*}{2500} & (Tgt Out) -1.3 & 7284 & 412 \\
\hline & & (Tgt Out) +1.3 & 2298 & 107 \\
\hline & \multirow[t]{2}{*}{-2500} & (Tgt In) -1.3 & 5851 & 329 \\
\hline & & $($ Tgt In $)+1.3$ & 5999 & 296 \\
\hline & \multirow[t]{2}{*}{2500} & (Tgt In) -1.3 & 6752 & 386 \\
\hline & & $($ Tgt In $)+1.3$ & 4939 & 242 \\
\hline \multirow[t]{14}{*}{-3300} & -2500 & (Tgt Out) +0.0 & 7103 & 334 \\
\hline & 2500 & (Tgt Out) +0.0 & 7011 & 322 \\
\hline & \multirow[t]{2}{*}{-2500} & (Tgt Out) -1.3 & 6795 & 351 \\
\hline & & (Tgt Out) +1.3 & 5181 & 201 \\
\hline & \multirow[t]{2}{*}{2500} & (Tgt Out) -1.3 & 7139 & 371 \\
\hline & & (Tgt Out) +1.3 & 3264 & 132 \\
\hline & \multirow[t]{4}{*}{-2500} & (Tgt In) -2.2 & 1601 & 150 \\
\hline & & (Tgt In) -1.3 & 6195 & 281 \\
\hline & & $($ Tgt In $)+1.3$ & 5919 & 256 \\
\hline & & $($ Tgt In) +2.2 & 3391 & 278 \\
\hline & \multirow[t]{4}{*}{2500} & (Tgt In) -2.2 & 1890 & 125 \\
\hline & & (Tgt In) -1.3 & 7319 & 357 \\
\hline & & (Tgt In) +1.3 & 3718 & 165 \\
\hline & & $($ Tgt In) +2.2 & 2250 & 125 \\
\hline
\end{tabular}

Table 4.5: Phase 1. Number of $\Omega^{-}$s for each beam condition. Odd conditions. 


\begin{tabular}{|c|c|c|c|c|c|}
\hline $\begin{array}{l}\text { Hyperon } \\
\text { Magnet } \\
\text { (Amps) }\end{array}$ & $\begin{array}{l}\text { BM109s } \\
\text { (Amps) }\end{array}$ & $\begin{array}{l}\text { Upstrm. } \\
\text { Tgting } \\
\text { (mR) }\end{array}$ & $\begin{array}{c}\text { Downstm. } \\
\text { Tgting } \\
0.0 \mathrm{mR} \\
\end{array}$ & $\begin{array}{c}\Omega^{-} \\
\text {Candidates } \\
\text { Tapped Out }\end{array}$ & $\begin{array}{c}\Omega^{-} \\
\text {Passed } \\
\text { All Cuts }\end{array}$ \\
\hline \multirow{6}{*}{-450} & \multirow{6}{*}{-2500} & -2.0 & \multirow{3}{*}{ (Tgt In) } & 76409 & 11989 \\
\hline & & +2.0 & & 69675 & 11406 \\
\hline & & +0.0 & & 83015 & 7638 \\
\hline & & -2.0 & \multirow[t]{3}{*}{ (Tgt Out) } & 1249 & 166 \\
\hline & & +2.0 & & 1179 & 168 \\
\hline & & +0.0 & & 2650 & 230 \\
\hline \multirow[t]{3}{*}{-750} & \multirow[t]{3}{*}{-2500} & -2.0 & \multirow[t]{3}{*}{ (Tgt In) } & 1619 & 289 \\
\hline & & +2.0 & & 1816 & 358 \\
\hline & & +0.0 & & 5116 & 527 \\
\hline \multirow[t]{5}{*}{-1000} & \multirow[t]{5}{*}{-2500} & -2.0 & \multirow[t]{5}{*}{ (Tgt In) } & 7699 & 1375 \\
\hline & & -1.0 & & 3019 & 355 \\
\hline & & +1.0 & & 5336 & 516 \\
\hline & & +2.0 & & 8196 & 1684 \\
\hline & & +0.0 & & 3373 & 429 \\
\hline
\end{tabular}

Table 4.6: Phase 2. Number of $\Omega^{-} \mathrm{s}$ for each beam condition. 


\subsection{Cascade Minus Event Selection and Background}

Samples of $\Xi^{-} \rightarrow \Lambda \pi^{-}$events from Phase 1 and Phase 2 were selected for use as a check of the $\Omega^{-}$results. As there were so many $\Xi^{-}$events in Phase 2 at the Hyperon Magnet setting of $-450 \mathrm{Amps}$, it was not practical or necessary to analyze every $\Xi^{-}$ event. A scheme was chosen which picked a sample with the same beam and detector characteristics as the $\Omega^{-}$sample. After every $\Omega^{-}$on the 3-track data summary tapes, for every run with the Hyperon Magnet set at -450 amps, the next fifteen events were selected for analysis as possible $\Xi^{-}$events. This selected about $12 \%$ of the whole $\Xi^{-}$ sample available at this condition. These events were written to separate files and provided a $\Xi^{-}$sample roughly $10 \times$ the $\Omega^{-}$sample after cuts. The other Hyperon Magnet settings provided far more $\Xi^{-}$than $\Omega^{-}$events but were managable without this selection process as there were fewer tapes and fewer events recorded under those conditions. A small fraction $(\sim 8 \%)$ of the Phase $1 \Xi^{-}$were selected from 2-4 runs at each of 5 Hyperon Magnet currents to provide a comparison with the $\Omega^{-}$sample.

A set of cuts similar to, but not as extensive as those used to isolate the $\Omega^{-}$sample, isolated the $\Xi^{-}$sample from these files. The same $\mathrm{p}-\pi^{-}$invariant mass cut, geometric $\chi^{2}$ cut, vertex cut, vertex separation cut, and target-pointing cut were used to determine the $\Xi^{-}$sample as were used for the $\Omega^{-}$. The cuts on the $\cos \theta$ distributions were not necessary. The $\Xi^{-}$background consisted of $\Omega^{-} \rightarrow \Xi^{-} \pi^{0}$ background events which remained after the target pointing cut and $\Omega^{-} \rightarrow \Lambda \mathrm{K}^{-}$events. The $\Omega^{-} \rightarrow \Xi^{-} \pi^{0}$ were negligible after the $\Xi^{-}$mass cut. Table 4.7 relates the cuts to a cut number which is used in Table 4.8 and showrs the fraction of 3-track triggers remaining after each cut. Table 4.8 shows an estimate of the number of $\Xi^{-}$events which were cut as well as the fraction of background events which remained as determined from a Monte Carlo study (M.C. was generated with the Hyperon Magnet at -450 Amps). Less than $.3 \%$ of the $\Xi^{-}$sample came from background events. The largest background was $\Omega^{-} \rightarrow \Lambda \mathrm{K}^{-}$. The $\mathrm{K}^{-} \rightarrow 3 \pi$ and $\Omega^{-} \rightarrow \Xi^{0} \pi^{-}$backgrounds were determined to be negligible. The $\Lambda-\pi^{-}$mass, geometric $\chi^{2}, \Xi^{-}$momentum, $\Xi^{-}$vertex and $\Lambda$ vertex are shown in Figures 


\begin{tabular}{||c|c|c||}
\hline $\begin{array}{c}\text { Cut } \\
\text { Number }\end{array}$ & $\begin{array}{c}\text { Cut } \\
\text { Description }\end{array}$ & $\begin{array}{c}\% \text { 3-trk Events } \\
\text { Remaining }\end{array}$ \\
\hline 0 & IOK=0 & 100. \\
\hline 1 & Decay vertex $\Xi^{-}>z=0 c m$ & 91.8 \\
\hline 2 & $\mathrm{R}^{2}<.40 \mathrm{~cm}^{2}$ & 77.4 \\
\hline 3 & Decay vertex $\Lambda>\Xi^{-}$ & 73.0 \\
\hline 4 & Momentum Range Cuts & 73.0 \\
\hline 5 & $1.106<m_{p \pi}<1.126 \mathrm{GeV} / \mathrm{c}^{2}$ & 72.1 \\
\hline 6 & $1.306<m_{\Lambda \pi}<1.338 \mathrm{GeV} / \mathrm{c}^{2}$ & 69.4 \\
\hline
\end{tabular}

Table 4.7: Summary of the cuts used to select the $\Xi^{-}$event sample. Including Phase 1

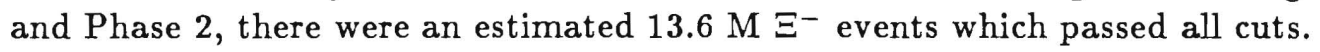

4.26 to 4.30 . Tables 4.9 and 4.10 show the number of $\Xi^{-}$events which passed all cuts for Phase 1 and Phase 2. 


\begin{tabular}{||c|c|c|c||}
\hline & $\begin{array}{c}\text { Scaled } \\
\Xi^{-} \rightarrow \Lambda \pi^{-} \\
\text {Monte Carlo } \\
\text { Events } \\
\text { Rut } \\
\text { Number }\end{array}$ & $\begin{array}{c}\text { Scaled } \\
\Omega^{-} \rightarrow \Lambda \mathrm{K}^{-} \\
\text {Monte Carlo } \\
\text { Events } \\
\text { Remaining }\end{array}$ & $\begin{array}{c}\text { Scaled } \\
\Omega^{-} \rightarrow \Xi^{-} \pi^{0} \\
\text { Monte Carlo } \\
\text { Events } \\
\text { Remaining }\end{array}$ \\
\hline 0 & $16.7 \mathrm{M}$ & 265130 & 108530 \\
\hline 1 & $15.1 \mathrm{M}$ & 214950 & 106710 \\
\hline 2 & $14.5 \mathrm{M}$ & 199170 & 21116 \\
\hline 3 & $13.8 \mathrm{M}$ & 192160 & 19980 \\
\hline 4 & $13.8 \mathrm{M}$ & 191940 & 19980 \\
\hline 5 & $13.6 \mathrm{M}$ & 190620 & 19750 \\
\hline 6 & $13.6 \mathrm{M}$ & 13584 & 19750 \\
\hline
\end{tabular}

Table 4.8: Summary of cuts used to select $\Xi^{-}$s. The $\Xi^{-}$sample was scaled to the estimated number of data $\Xi^{-} \rightarrow \Lambda \pi^{-}$. The $\Omega^{-}$sample was scaled to the estimated number of data $\Omega^{-} \rightarrow \Lambda K^{-}$. 


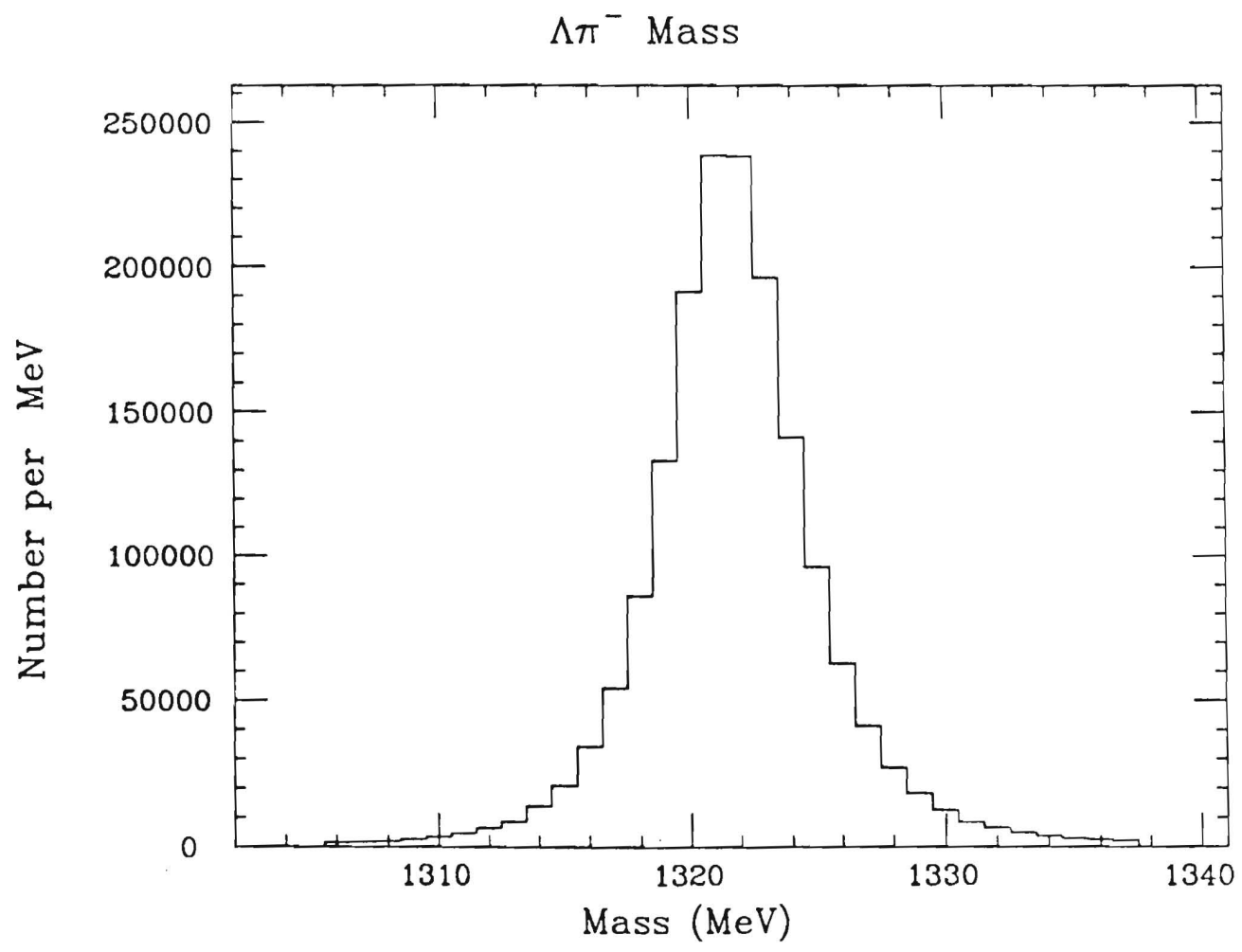

Figure 4.26: Lambda $-\pi^{-}$invariant mass distribution. 


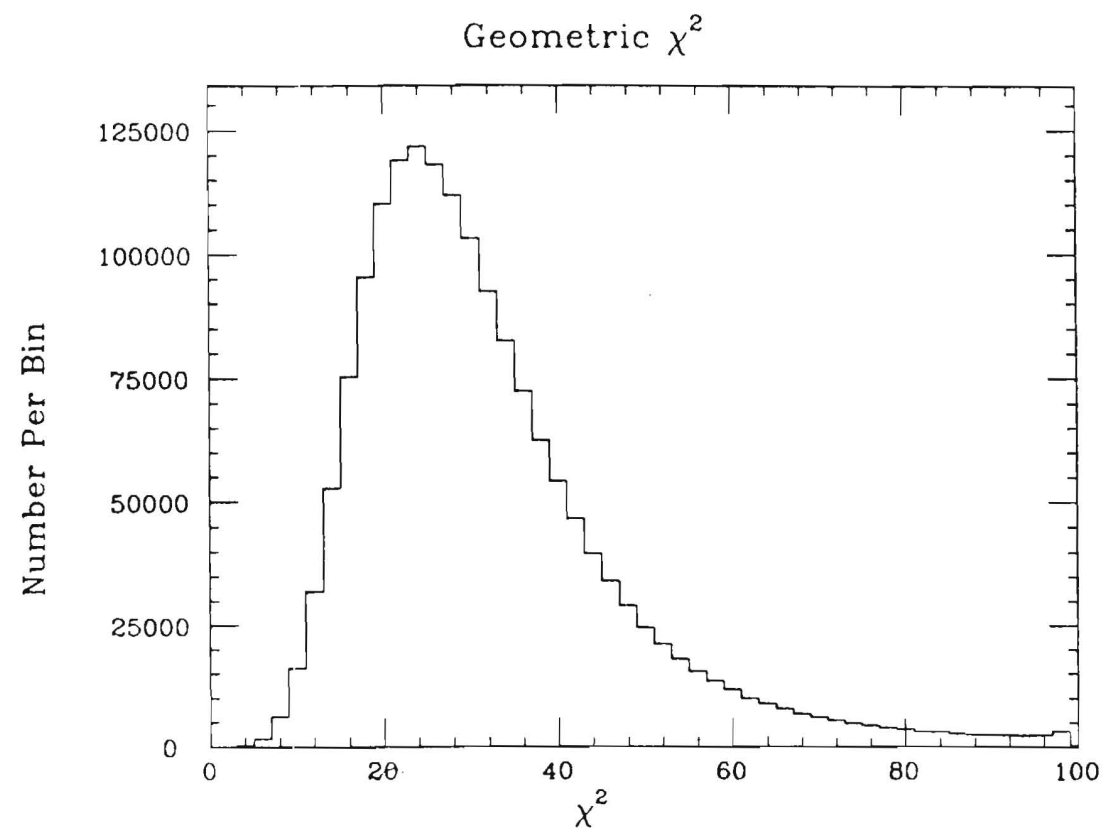

Figure 4.27: Geometric $\chi^{2}$ for $\Xi^{-}$events.

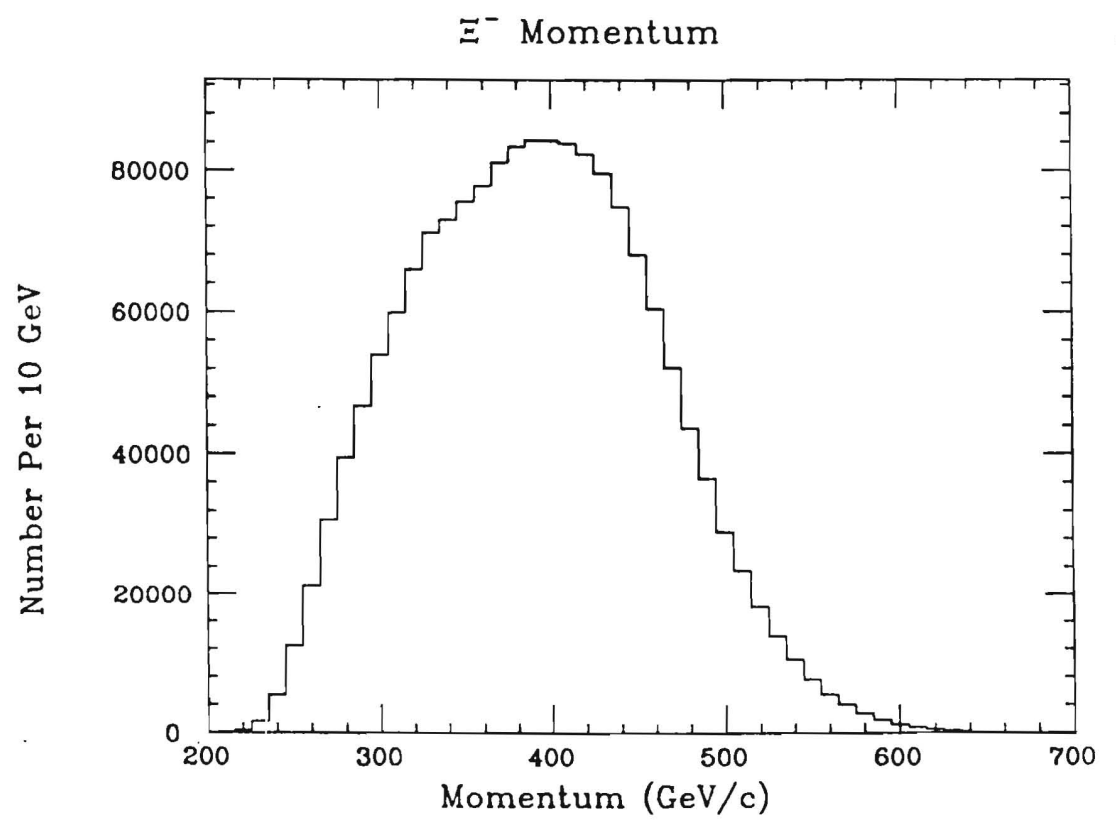

Figure 4.28: $\Xi^{-}$momentum distribution. 


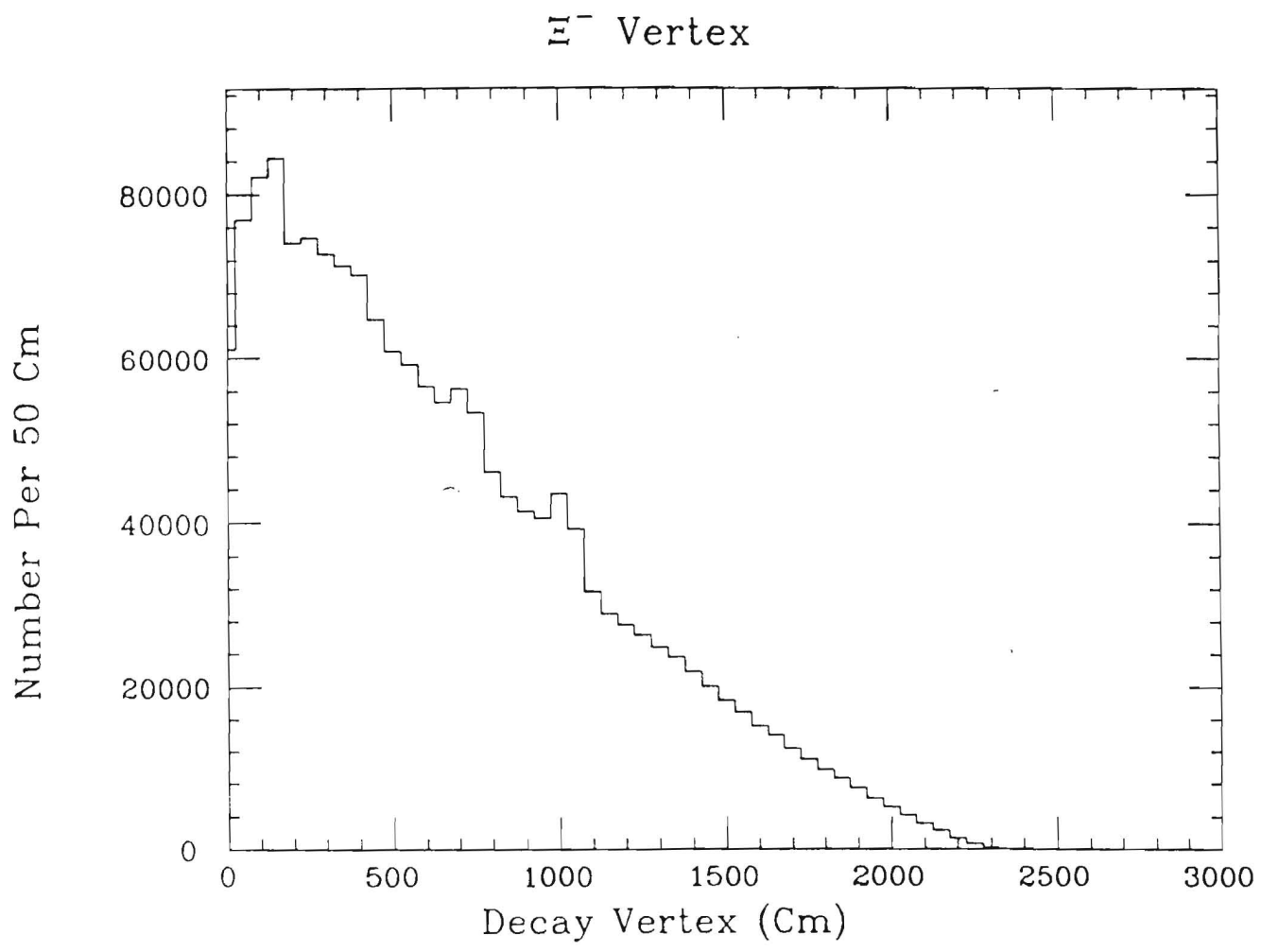

Figure 4.29: $\Xi^{-}$vertex distribution. 


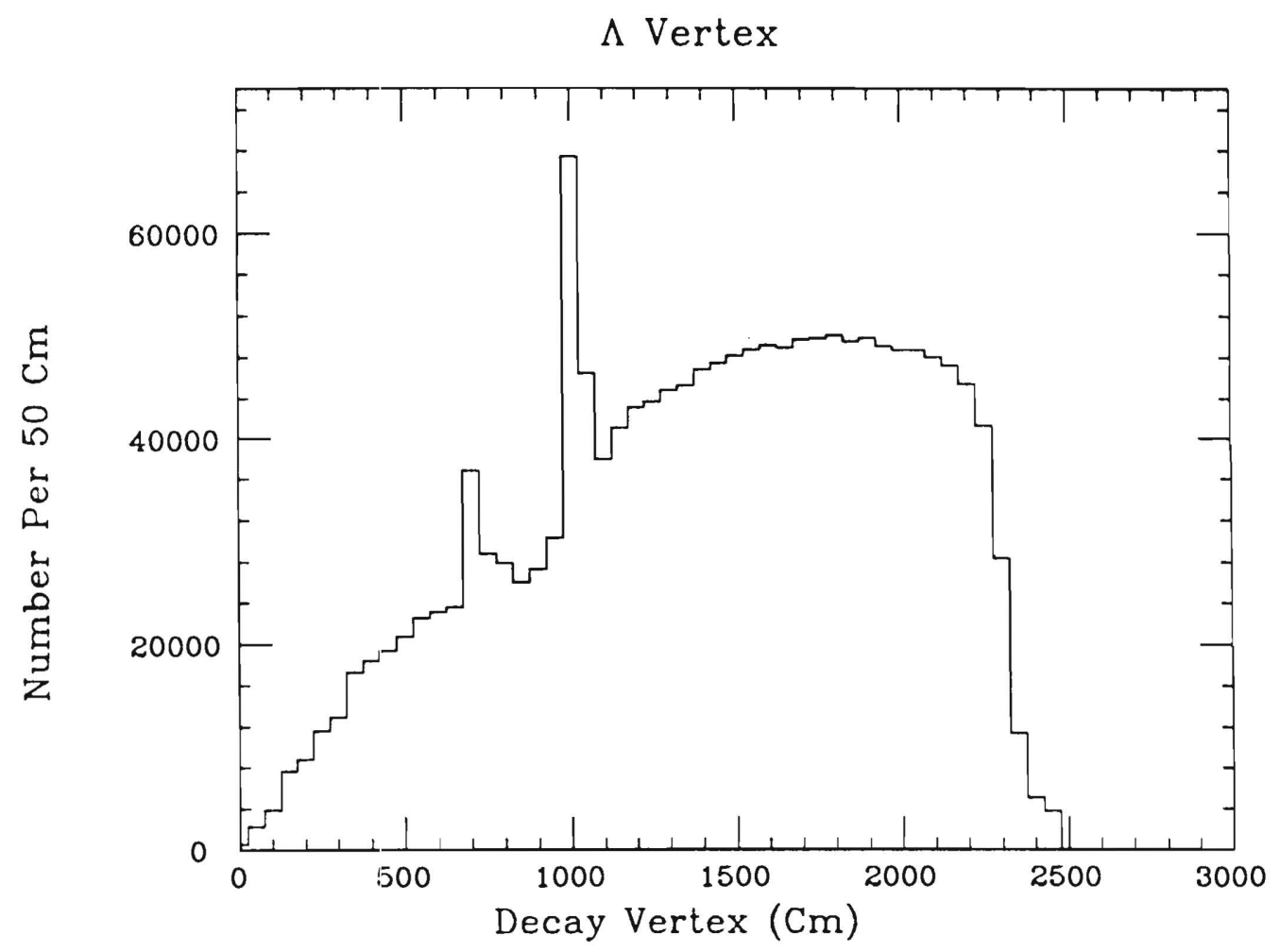

Figure 4.30: $\Lambda$ vertex distribution. 


\begin{tabular}{||c|c|c|c|c||}
\hline $\begin{array}{c}\text { Hyperon } \\
\text { Magnet } \\
\text { (Amps) }\end{array}$ & $\begin{array}{c}\text { BM109s } \\
(\text { Amps })\end{array}$ & $\begin{array}{c}\text { Tgting } \\
\text { Angle } \\
(\mathrm{mR})\end{array}$ & $\begin{array}{c}\Xi^{-} \\
\text {Candidates } \\
\text { Analyzed }\end{array}$ & $\begin{array}{c}\Xi^{-} \\
\text {Passed } \\
\text { All Cuts }\end{array}$ \\
\hline-500 & -2500 & -2.5 & 180080 & 124298 \\
\cline { 3 - 5 } & & +2.5 & 170537 & 116973 \\
\hline-1000 & -2500 & -2.5 & 107611 & 73423 \\
\cline { 3 - 5 } & & +2.5 & 169960 & 115825 \\
\hline-1700 & -2500 & -2.5 & 162621 & 109211 \\
\cline { 3 - 5 } & & +2.5 & 201171 & 134376 \\
\hline-2500 & \multirow{2}{*}{-2500} & -2.5 & 158975 & 104048 \\
\cline { 3 - 5 } & & +2.5 & 149804 & 97602 \\
\hline-3300 & -2500 & -2.5 & 132209 & 82659 \\
\cline { 3 - 5 } & & +2.5 & 82545 & 52524 \\
\hline
\end{tabular}

Table 4.9: Phase 1. Number of $\Xi^{-} \mathrm{s}$ for each beam condition. This is a sample of $\sim 8 \%$ of the available $\Xi^{-}$. 


\begin{tabular}{|c|c|c|c|c|c|}
\hline $\begin{array}{c}\text { Hyperon } \\
\text { Magnet } \\
\text { (Amps) }\end{array}$ & $\begin{array}{l}\text { BM109s } \\
\text { (Amps) }\end{array}$ & $\begin{array}{c}\text { Upstrm. } \\
\text { Tgting } \\
\text { (mR) }\end{array}$ & $\begin{array}{c}\text { Downstm. } \\
\text { Tgting } \\
0.0 \mathrm{mR} \\
\end{array}$ & $\begin{array}{c}\Xi^{-} \\
\text {Candidates } \\
\text { Analyzed }\end{array}$ & $\begin{array}{c}\Xi^{-} \\
\text {Passed } \\
\text { All Cuts }\end{array}$ \\
\hline \multirow[t]{6}{*}{-450} & \multirow[t]{6}{*}{-2500} & -2.0 & \multirow[t]{3}{*}{ (Tgt In) } & 156587 & 117166 \\
\hline & & +2.0 & & 147921 & 110589 \\
\hline & & +0.0 & & 101637 & 70366 \\
\hline & & -2.0 & \multirow[t]{3}{*}{ (Tgt Out) } & 20237 & 14533 \\
\hline & & +2.0 & & 16917 & 12023 \\
\hline & & +0.0 & & 40225 & 27230 \\
\hline \multirow[t]{3}{*}{-750} & \multirow[t]{3}{*}{-2500} & -2.0 & \multirow[t]{3}{*}{ (Tgt In) } & 28513 & 21276 \\
\hline & & +2.0 & & 30004 & 22311 \\
\hline & & +0.0 & & 87245 & 59765 \\
\hline \multirow[t]{5}{*}{-1000} & \multirow[t]{5}{*}{-2500} & -2.0 & \multirow[t]{5}{*}{ (Tgt In) } & 123961 & 90945 \\
\hline & & -1.0 & & 60138 & 43559 \\
\hline & & +1.0 & & 53009 & 38207 \\
\hline & & +2.0 & & 139268 & 102964 \\
\hline & & +0.0 & & 89223 & $612 \overline{26}$ \\
\hline
\end{tabular}

Table 4.10: Phase 2. Number of $\Xi^{-} \mathrm{s}$ for each beam condition. The Hyperon Magnet $=-450$ Amps sample is about $12 \%$ of all the $\Xi^{-}$available at that condition. 


\section{Chapter 5}

\section{Polarization}

This chapter concentrates on the $\Xi^{-}$and $\Omega^{-}$polarization analysis and it's results. The first section contains a derivation relating the $\Omega^{-}$polarization and the daughter- $\Lambda$ polarization. This relation is important because the distribution of protons in the $\Lambda$ rest frame provided a measurement of the daughter- $\Lambda$ polarization which was then used to calculate the $\Omega^{-}$and $\Xi^{-}$polarization. The next section contains a discussion of the Hybrid Monte Carlo, which was used to measure the $\Lambda$ polarization from the $\Omega^{-}$ and $\Xi^{-}$decays. The next section contains Phase $1 \Xi^{-}$polarization results. The next section contains Phase $1 \Omega^{-}$polarization measurements. First, the results are of the Hybrid Monte Carlo are presented. Then the magnetic moment found in Phase 2 is used in a "grand" $\chi^{2}$ fit to the Phase 1 polarization data. The next two sections contain the results of the $\Xi^{-}$and $\Omega^{-}$polarization measurements of Phase 2. Finally, the last section describes Monte Carlo checks of the procedure.

\subsection{The Joint Angular Decay Distribution and Polarization}

This section contains an outline of a derivation relating the polarization of the $\Omega^{-}$and $\Xi^{-}$to the polarization of the daughter $\Lambda \mathrm{s}$. The results are applicable to the decay of any spin-J baryon into a spin- $1 / 2$ baryon and a spin-0 meson. There are two reasons for maintaining this generality. First, the spin of the $\Omega^{-}$has never been proven to be $3 / 2$. Second, the results are applicable to both the $\Omega^{-}$and the $\Xi^{-}$decays.

Two coordinate systems are defined in order to determine the polarization and angular distribution of the decay products of a particle with spin-J. The first is an arbitrary coordinate system, $S$, defined in the $\Omega^{-}$rest frame. The laboratory axes are suitable. 
That is:

$$
S=(\hat{x}, \hat{y}, \hat{z})
$$

where the quantization axis is $\hat{z}$. The other coordinate system, $S^{\prime}$, also defined in the $\Omega^{-}$rest frame, is

$$
S^{\prime}=(\hat{X}, \hat{Y}, \hat{\Lambda})
$$

where $\hat{\Lambda}$ is the unit vector in the direction of the $\Lambda$ momentum,

$$
\hat{X}=\frac{\hat{\Lambda} \times(\hat{\Lambda} \times \hat{z})}{|\hat{\Lambda} \times(\hat{\Lambda} \times \hat{z})|}
$$

and

$$
\hat{Y}=\frac{\hat{z} \times \hat{\Lambda}}{|\hat{z} \times \hat{\Lambda}|}
$$

$S^{\prime}$ is also sometimes called the $\Lambda$ helicity frame. $S^{\prime}$ changes from event to event, depending on the decay angles. Figure 5.1 shows the orientation of the two coordinate systems.

The daughter baryons $(\Lambda \mathrm{s})$ have the following angular distribution and polarization components:

$$
\begin{gathered}
I(\theta, \phi)=A(\theta, \phi)+\alpha_{\Omega} B(\theta, \phi), \\
I(\theta, \phi) \vec{P}_{\Lambda} \cdot \hat{\Lambda}=\alpha_{\Omega} A(\theta, \phi)+B(\theta, \phi),
\end{gathered}
$$

and

$$
\begin{gathered}
I(\theta, \phi) \vec{P}_{\Lambda} \cdot(\hat{X}+i \hat{Y})=(i \beta-\gamma)(2 J+1) \sum_{L=1 \text { odd }}^{2 J} \sum_{M=-L}^{L} \\
\sqrt{\frac{2 L}{4 \pi}} n_{L 0}^{J} t_{L M}(L(L+1))^{-1 / 2} D_{M 1}^{L}(\phi, \theta, 0),
\end{gathered}
$$




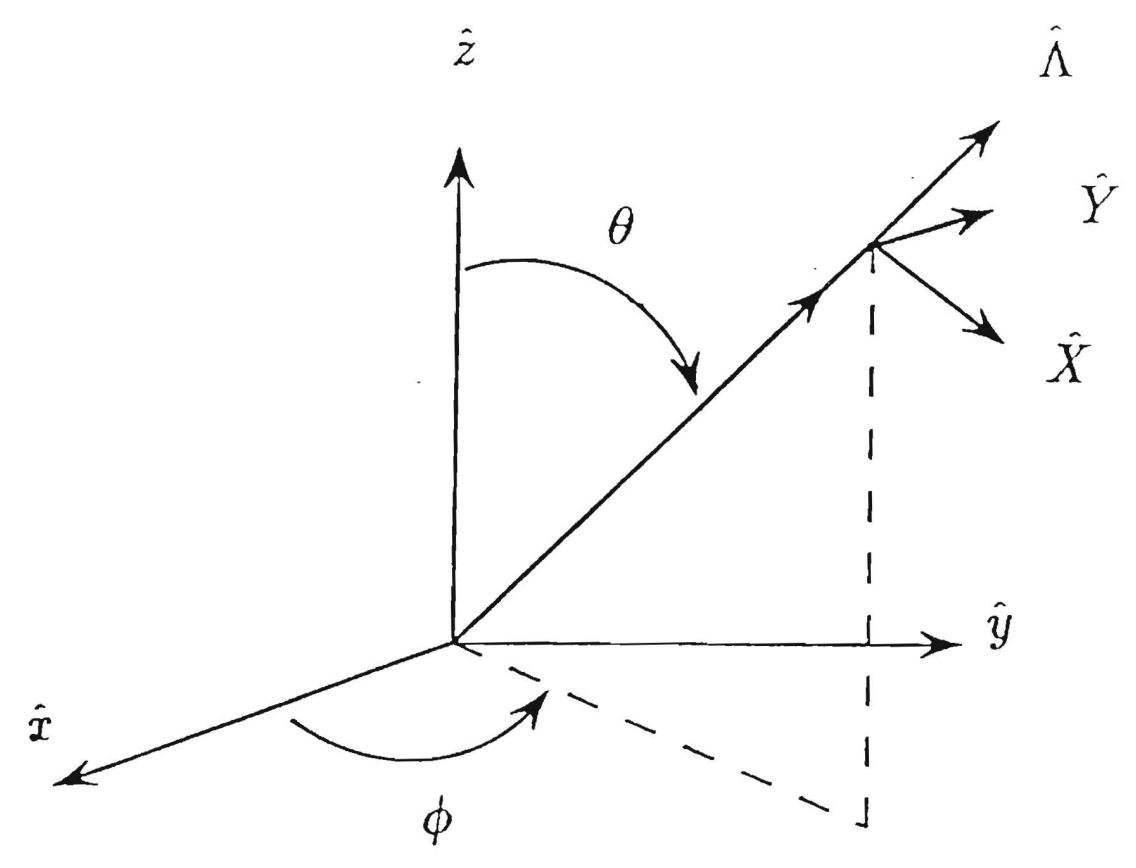

Figure 5.1: Relative orientation of coordinate systems $S$ and $S^{\prime}$.

where,

$$
\begin{aligned}
& A(\theta, \phi)=\sum_{L=0 \text { even }}^{2 J-1} \sum_{M=-L}^{L} n_{L 0}^{J} t_{L M} Y_{L M}^{*}(\theta, \phi) \\
& B(\theta, \phi)=\sum_{L=1 \text { odd }}^{2 J} \sum_{M=-L}^{L} n_{L 0}^{J} t_{L M} Y_{L M}^{*}(\theta, \phi) .
\end{aligned}
$$

The angles $\theta$ and $\phi$ are the polar angles of the daughter baryon in the parent rest frame. $Y_{L M}$ and $D_{M 1}^{L}$ are the spherical harmonics and the Wigner rotation matrices, respectively. The $n_{L 0}^{J}$ are normalization constants proportional to Clebsh-Gordan coefficients and are given by

$$
n_{L 0}^{J}=(-1)^{J-1 / 2} \sqrt{\frac{2 J+1}{4 \pi}}\left\langle J J \frac{1}{2} \frac{1}{2} \mid L 0\right\rangle .
$$

The $t_{L M}$ are the elements of the spin-density matrix related to the expectation values of the spin components of the parent baryon. In particular, the $\Omega^{-}$(or $\Xi^{-}$) vector 
(rank 1) polarization components in terms of the $t_{1 M}$ (where $M=-1,0,1$ ) are

$$
\begin{gathered}
P_{x}=\sqrt{\frac{J+1}{J}}\left(\frac{t_{1-1}-t_{11}}{\sqrt{2}}\right) \\
P_{y}=i \sqrt{\frac{J+1}{J}}\left(\frac{t_{1-1}+t_{11}}{\sqrt{2}}\right) \\
P_{z}=\sqrt{\frac{J+1}{J}} t_{10} .
\end{gathered}
$$

Furthermore, $t_{00}$ is normalized to 1 and $t_{L-M}=(-1)^{M} t_{L}^{*}$. The higher rank polarizations are discussed further in Appendix B.

The $\Lambda$ s decay via the weak interaction. The main decay mode is $\Lambda \rightarrow p \pi^{-}$, with a branching fraction of $64 \%$. The distribution of the protons is given by

$$
\frac{1}{N_{p}} \frac{d N_{p}}{d \Omega_{p}}=\frac{1}{4 \pi}\left(1+\alpha_{\Lambda} \vec{P}_{\Lambda} \cdot \hat{p}\right)
$$

where $\Omega_{p}, \hat{p}$ and $\vec{P}_{\Lambda}$ are, respectively, the solid angle, the unit vector in the direction of the proton momentum, and the polarization of the $\Lambda \mathrm{s}$, all in the $\Lambda$ rest frame. $\alpha_{\Lambda}$ is measured as $+.642 \pm .013$. As Equation 5.11 is independent of azimuthal angle, it can be rewritten as

$$
\frac{1 .}{N_{p}} \frac{d N_{p}}{d \cos \theta_{p}}=\frac{1}{2}\left(1+\alpha_{\Lambda} \vec{P}_{\Lambda} \cdot \hat{n} \cos \theta_{p}\right)
$$

where $\hat{n}$ is an arbitrary unit vector and $\cos \theta_{p}$ is $\hat{n} \cdot \hat{p}$. Choosing $\hat{n}$ parallel to the laboratory axes, the $\Lambda$ polarization can be determined. Choosing $\hat{n}$ parallel to the $\Lambda$ momentum in the $\Omega^{-}$rest frame, the $\Lambda$ helicity can be determined.

The joint angular distribution of $\Omega^{-}$decay products is $I_{\Lambda}\left(\theta_{\Lambda}, \phi_{\Lambda}\right) I_{p}\left(\theta_{p}, \phi_{p}\right)$. This can be written

$$
\begin{gathered}
I_{\Lambda}\left(\theta_{\Lambda}, \phi_{\Lambda}\right) I_{p}\left(\theta_{p}, \phi_{p}\right)=\frac{1}{4 \pi}\left(I_{\Lambda}+\alpha_{\Lambda} I_{\Lambda} \vec{P}_{\Lambda} \cdot \hat{\Lambda} \hat{\Lambda} \cdot \hat{p}\right. \\
\left.+\alpha_{\Lambda} I_{\Lambda} \vec{P}_{\Lambda} \cdot \hat{X} \hat{X} \cdot \hat{p}+\alpha_{\Lambda} I_{\Lambda} \vec{P}_{\Lambda} \cdot \hat{Y} \hat{Y} \cdot \hat{p}\right) .
\end{gathered}
$$

Expressing the proton distribution in $S^{\prime}$, as shown in Figure 5.2, 


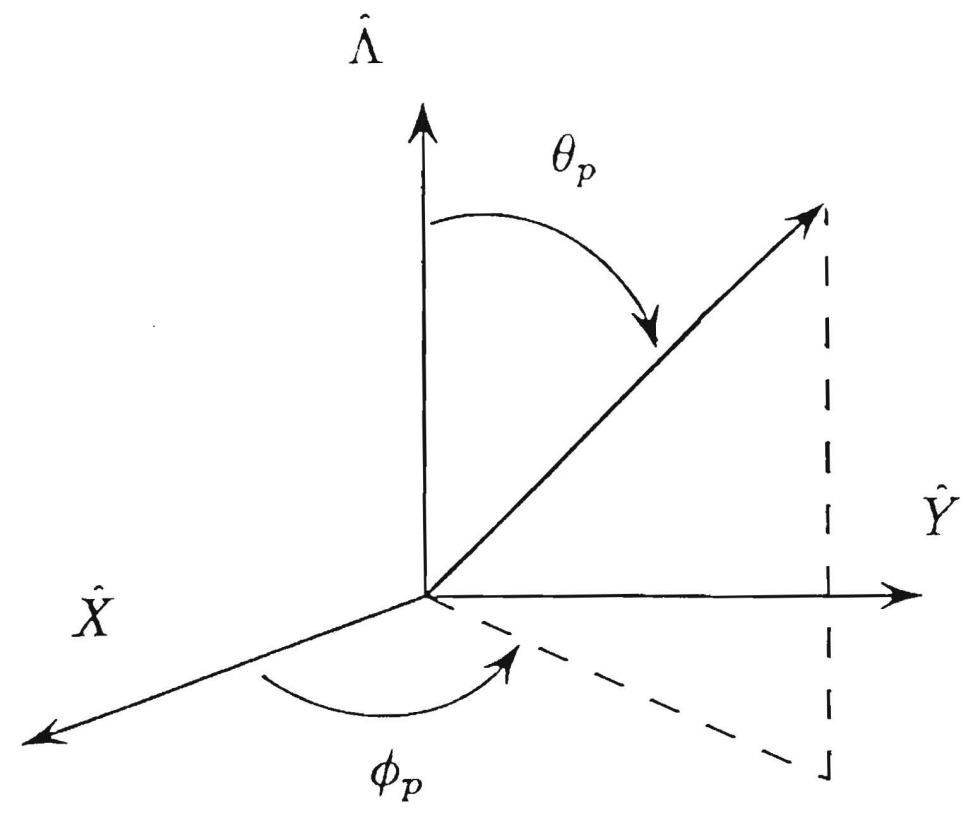

Figure 5.2: Proton angles in the $\Lambda$ rest frame.

$$
\begin{aligned}
\hat{\Lambda} \cdot \hat{p} & =\cos \theta_{p} \\
\hat{X} \cdot \hat{p} & =\sin \theta_{p} \cos \phi_{p} \\
\hat{Y} \cdot \hat{p} & =\sin \theta_{p} \cos \phi_{p}
\end{aligned}
$$

and integrating Equation 5.13 over $\phi_{p}$ and $\Omega_{\Lambda}$ leaves an expression for the proton distribution. It is

$$
\frac{1}{N_{p}} \frac{d N_{p}}{d \cos \theta_{p}}=1 / 2\left(1+\alpha_{\Omega} \alpha_{\Lambda} \cos \theta_{p}\right)
$$

Noticing Equation 5.12 implies $d N_{p} / d \cos \theta_{p}=1 / 2\left(1+\alpha_{\Lambda} \vec{P}_{\Lambda} \cdot \hat{\Lambda} \cos \theta_{p}\right)$ leads to the relation

$$
\alpha_{n}=\vec{P}_{\Lambda} \cdot \hat{\Lambda}
$$

on comparison with Equation 5.15. This relation was used to measure $\alpha_{\Omega}$ [4]. 
Another expression is available for the proton distribution. Projecting the $S^{\prime}$ coordinate system on $S$ gives

$$
\left(\begin{array}{c}
\hat{\Lambda} \\
\hat{X} \\
\hat{Y}
\end{array}\right)=\left(\begin{array}{ccc}
\sin \theta_{\Lambda} \cos \phi_{\Lambda} & \sin \theta_{\Lambda} \sin \phi_{\Lambda} & \cos \theta_{\Lambda} \\
\cos \theta_{\Lambda} \cos \phi_{\Lambda} & \cos \theta_{\Lambda} \sin \phi_{\Lambda} & -\sin \theta_{\Lambda} \\
-\sin \phi_{\Lambda} & \cos \phi_{\Lambda} & 0
\end{array}\right)\left(\begin{array}{c}
\hat{z} \\
\hat{x} \\
\hat{y}
\end{array}\right) \text {. }
$$

Substituting for $\hat{\Lambda}, \hat{X}$, and $\hat{Y}$ in Equation 5.13 and again integrating over $\phi_{p}$ and $\Omega_{\Lambda}$ yields the second expression for the proton distribution. It is

$$
\frac{1}{N_{p}} \frac{d N_{p}}{d \cos \theta_{p}}=\frac{1}{2}\left(1+\frac{\alpha_{\Lambda}}{2(J+1)}\{1+\gamma(2 J+1)\} \vec{P}_{\Omega^{-}} \cdot \hat{p}\right)
$$

Comparing Equation 5.12 or 5.11 with Equation 5.17 yields an expression relating the $\Omega^{-}$vector polarization to the $\Lambda$ polarization. It is

$$
\vec{P}_{\Lambda}=\frac{\{1+\gamma(2 J+1)\}}{2(J+1)} \vec{P}_{\mathbf{n}^{-}} .
$$

$\gamma_{\Omega}$ is unknown, but as $\alpha_{\Omega^{-}}(=0.026 \pm 0.026)$ is close to zero[9], $\left|\gamma_{\Omega^{-}}\right| \approx 1$. If $\gamma_{\Omega^{-}}=+1$, $\vec{P}_{\Lambda}=\vec{P}_{\Omega^{-}}$, independent of the $\Omega^{-}$spin. If $\gamma_{\Omega^{-}}=-1, \vec{P}_{\Lambda}=-\frac{J}{J+1} \vec{P}_{\Omega^{-}}$. Using $J_{\Omega^{-}}=\frac{3}{2}$, Equation 5.18 becomes

$$
\vec{P}_{\Lambda}=\left\{\begin{aligned}
\vec{P}_{n^{-}} & \text {if } \gamma_{n^{-}}=1, \\
-0.6 \vec{P}_{\mathbf{n}^{-}} & \text {if } \gamma_{\mathbf{n}^{-}}=-1
\end{aligned}\right.
$$

For the $\Xi^{-}$where $J=\frac{1}{2}$ and $\gamma_{\Xi^{-}}=0.89[9]$, Equation 5.18 becomes

$$
\vec{P}_{\Lambda}=0.93 \vec{P}_{\Xi^{-}}
$$

Therefore, the $\Omega^{-}$and $\Xi^{-}$polarizations are obtained by measuring the $\Lambda$ polarization, by means of the asymmetry of the proton distribution in the $\Lambda$ rest frame, and using the appropriate relation from Equations 5.19 and 5.20 to relate the $\Lambda$ polarization to the $\Omega^{-}$or $\Xi^{-}$polarization. This method was used by Bunce [43] in measuring the polarization and magnetic moment of the $\Xi^{0}$. This generalization, Equation 5.18 , of the relation between the polarization of the spin-1/2 daughter particles and the spin-J baryon is due to the work of Luk[44]. 


\subsection{The Hybrid Monte Carlo}

The components of the $\Lambda$ polarization parallel to the laboratory axes $\hat{x}, \hat{y}$ and $\hat{z}$ were measured by finding the asymmetry of the proton distribution in the $\Lambda$ rest frame. However, the asymmetry of the proton distribution of Equation 5.12 was modified by the acceptance, $\mathcal{A}\left(\cos \theta_{p}\right.$, kinematic variables $)$, of the apparatus and reconstruction algorithm. Because this acceptance is not a constant function in $\cos \theta_{p}$, Equation 5.12 becomes

$$
\frac{1}{N_{p}} \frac{d N_{p}}{d \cos \theta_{p}}=\frac{1}{2} \mathcal{A}\left(\cos \theta_{p}, \text { kinematic variables }\right)\left(1+\alpha_{\Lambda} \vec{P}_{\Lambda} \cdot \hat{n} \cos \theta_{p}\right) .
$$

This precluded measuring the polarization at each targeting angle simply by measuring the slope on a histogram of the $\cos \theta_{p}$ distributions. That contribution of the acceptance to the slope is called the bias. However, because the polarization changes sign with production angle, if $\mathcal{A}$ were identical for positive and negative production angles, the polarization could be found by measuring the slope of

$$
\alpha_{\Lambda} \vec{P}_{\Lambda} \cos \theta_{p}=\left(\frac{1}{N_{p}^{+}} \frac{d N_{p}^{+}}{d \cos \theta_{p}}-\frac{1}{N_{p}^{-}} \frac{d N_{p}^{-}}{d \cos \theta_{p}}\right) /\left(\frac{1}{N_{p}^{+}} \frac{d N_{p}^{+}}{d \cos \theta_{p}}+\frac{1}{N_{p}^{-}} \frac{d N_{p}^{-}}{d \cos \theta_{p}}\right)
$$

binned in $\cos \theta_{p}$. The superscript \pm indicates the production angle. The bias cancels. Preliminary results of E756 have been reported [45] using this method of determining the polarization of the $\Lambda \mathrm{s}$ from $\Omega^{-}$decays.

Rather than relying on the spectrometer acceptance cancellation, a Hybrid Monte Carlo was used to measure the polarization components, independently, at each targeting angle by determining the acceptance as a function of $\cos \theta$. Whereas a standard Monte Carlo required simulation of all the features of the data, including those incidental to the polarization measurement, the Hybrid Monte Carlo required only a model of the apparatus and trigger to mimic the acceptance. The Hybrid Monte Carlo generated "fake" events where random values of $\cos \theta_{p}$ between -1 and +1 replaced the $\cos \theta_{p}$ value of the polarization component under study. The other parameters of the real event, such as the primary momentum and vertex, the $\Lambda$ momentum and vertex, and the other decay angles of the proton were not changed. 
All events, fake and real, are required to pass the software model of the spectrometer and trigger. The fake events were boosted to the lab frame and traced through the spectrometer. The apertures were defined by the bottom of the downstream end of the upstream calorimeter, the top of $\mathrm{C} 7$, the trigger boundaries in $\mathrm{C8}$ and $\mathrm{C} 9$, the magnet apertures, and the boundaries of the $\mathrm{x}$-view of C8. The $y$-view apertures were restricted slightly more than the actual aperture because the aperture of the upstream calorimeter is at it's downstream end. All particles were required to fall within these apertures. This reduced the size of the event samples.

Fake events are generated for each real event until 10 fake events are accepted. If less than 10 fake events are accepted in 200 trys, the real event and the fake event are discarded. With this procedure, the Hybrid Monte Carlo "measures" the acceptance of the spectrometer and trigger. A disadvantage of the Hybrid Monte Carlo is that the acceptance of the reconstruction algorithm is not measured. It is approximated with a cut on the event topology and finally is removed by comparing the polarization measured at opposite production angles.

The polarization is measured by assigning to the $\mathrm{j}$-th Monte Carlo event of the $\mathrm{i}$-th real event a weight

$$
W_{i j}(k)=\frac{\left(1+\alpha_{\Lambda} \vec{P}_{\Lambda} \cdot \hat{n} \cos \theta_{i j}\right)}{\left(1+\alpha_{\Lambda} \vec{P}_{\Lambda} \cdot \hat{n} \cos \theta_{i}\right)}
$$

$\vec{P}_{\Lambda}$ is chosen by minimizing the $\chi^{2}$ deviation of the $\cos \theta_{p}$ distributions of the fake events from the data. The $\chi^{2}$ is calculated with

$$
\chi^{2}=\sum_{k=1}^{20}\left(N_{k}^{R}-\frac{N_{k}^{M C}}{10}\right)^{2} / N_{k}^{R},
$$

where $N_{k}^{R}$ is the sum of the real events and $N_{k}^{M C}=\sum_{i j} W_{i j}(k)$ is the sum of the fake events with $\cos \theta_{p}$ in bin k. For more details about the Hybrid Monte Carlo method see Ref. [46].

The polarizations of the two targeting angles at a given Hyperon Magnet field were measured with the Hybrid Monte Carlo. The polarization of the daughter $\Lambda \mathrm{s}$ and the 
bias were found adding and subtracting the two signals. That is:

$$
\alpha_{\Lambda} P_{\Lambda}=\frac{\alpha_{\Lambda} P_{\Lambda}^{+}-\alpha_{\Lambda} P_{\Lambda}^{-}}{2}
$$

and

$$
B=\frac{\alpha_{\Lambda} P_{\Lambda}^{+}+\alpha_{\Lambda} P_{\Lambda}^{-}}{2}
$$

where the superscript \pm indicates the targeting angle.

The asymmetry found when the protons are incident at $0 \mathrm{mR}$ was another measure of the bias. The transverse polarization is zero because there is no production plane to provide a preferred direction. Thus any measured signal must be bias and should be consistent with the bias determined from the data produced at targeting angles.

\subsection{Phase 1 Omega Minus Polarization Results}

This section contains a summary of the results of the Hybrid Monte Carlo study of the $\Omega^{-}$data of Phase 1 . These results are then combined with the magnetic moment result of Phase 2 to estimate the polarization at the target in Phase 1.

The event sample was futher reduced by a cut which insured that all the $\Omega^{-}$s precessed for the full length of the Hyperon Magnet. Monte Carlo studies showed that the error in the $\Omega^{-}$vertex at this $\mathrm{z}$-position was approximately $25 \mathrm{~cm}$. A cut on the primary vertex position required the $\Omega^{-}$decay at least $50 \mathrm{~cm}$ downstream of the Hyperon Magnet exit. However, cuts to match the acceptance of the reconstruction program were not used in the $\Omega^{-}$analysis because they cut $\sim 20 \%$ of the events from the already small samples. Instead, the measurement relies on cancellation of the reconstruction acceptance.

The data recorded with the BM109s set at -2500 Amps was analyzed separately from the data recorded with the BM109s set at +2500 Amps. This was done because the acceptance for the +2500 Amp sample was roughly $15 \%$ lower, based on the $\Omega^{-}$yield per proton. The acceptance was different for a combination of reasons. The particle distributions in the spectrometer upstream of the BM109s were identical. The beam 
exited the charged particle collimator with a position-momentum correlation such that the direction towards $+\hat{x}$ contained higher momentum particles. After the $\Omega^{-}$decayed, protons which came from the higher momentum primaries were weighted to the $+\hat{x}$ side. But, when the BM109s were set at $+2500 \mathrm{Amps}$, the protons were bent towards the $-\hat{x}$ side of the downstream chambers. In order to cross to the trigger side of $\mathrm{C} 9$, the stiffer protons had further to travel than the softer protons. The opposite is so when the BM109s were set at -2500 Amps. To complicate matters, C8 and C9 were offset slightly to the $-\hat{x}$ side in order to maximize the efficiency of the trigger when the BM109s were set at -2500 Amps. This offset was about $3.0 \mathrm{~cm}$ at $\mathrm{C} 8$ and $4.3 \mathrm{~cm}$ at C9. Stiff protons could not cross the C9 trigger boundary in the +2500 Amp sample. Furthermore, more soft pions from $\Lambda$ decay were lost in the +2500 Amp data than in the -2500 Amp data because the offset left less room on the $+\hat{x}$ side of C8. Because the acceptance is different, the data sets were analyzed independently. The results are combined in the grand fit.

The results from the Hybrid Monte Carlo are as follows. The asymmetry $\left(=\alpha_{\Lambda} P_{\Lambda}\right)$ for each of the conditions is shown in Table 5.1. These include results from the five Hyperon Magnet currents, at each of two BM109 currents used in Phase 1, for each of two $( \pm 2.5 \mathrm{mR})$ targeting angles. The components of the polarization are shown in Table 5.2. $P_{x}$ vs. $P_{z}$ is plotted in Figure 5.3. Table 5.3 shows the bias as determined from the $\pm 2.5 \mathrm{mR}$ data. All of the main conditions of Table 4.4, with the exception of the $0 \mathrm{mR}$ samples which w'ere too small, were analyzed independently. Some of the like conditions of Table 4.5 were grouped together because of the small number of events. Table 5.4 shows the Hybrid Monte Carlo fit statistics which include the final event sample sizes, the average momentum of the $\Omega^{-} \mathrm{s}$, and the $\chi^{2}$ of the data vs. Hybrid Monte Carlo $\cos \theta$ matchups. Polarization results are presented as $P_{\Omega}$ rather than $P_{\Lambda}$ with the assumption that $\gamma_{\Omega}=+1$. In other words, the factor of $\alpha_{\Lambda}(=0.642)$ has been removed from the results of Equation 5.25. Bias results are presented just as calculated with Equation 5.26. 


\begin{tabular}{|c|c|c|c|c|c|}
\hline $\begin{array}{l}\text { Hyperon } \\
\text { Magnet } \\
\text { (Amps) }\end{array}$ & $\begin{array}{l}\text { BM109 } \\
\text { Current } \\
\text { (Amps) }\end{array}$ & $\begin{array}{l}\text { Tgting } \\
\text { Angle } \\
(\mathrm{mR})\end{array}$ & $\begin{array}{l}\text { Asymmetry } \\
\hat{x} \\
\text { Component }\end{array}$ & $\begin{array}{c}\text { Asymmetry } \\
\hat{y} \\
\text { Component }\end{array}$ & $\begin{array}{c}\text { Asymmetry } \\
\hat{z} \\
\text { Component }\end{array}$ \\
\hline \multirow[t]{4}{*}{-500} & \multirow[t]{2}{*}{-2500} & +2.5 & $-0.006 \pm 0.031$ & $0.034 \pm 0.034$ & $0.118 \pm 0.036$ \\
\hline & & -2.5 & $-0.009 \pm 0.030$ & $0.011 \pm 0.033$ & $0.077 \pm 0.036$ \\
\hline & \multirow[t]{2}{*}{+2500} & +2.5 & $-0.028 \pm 0.030$ & $-0.006 \pm 0.034$ & $0.179 \pm 0.034$ \\
\hline & & -2.5 & $0.085 \pm 0.033$ & $0.021 \pm 0.036$ & $0.145 \pm 0.039$ \\
\hline \multirow[t]{4}{*}{-1000} & \multirow[t]{2}{*}{-2500} & +2.5 & $-0.024 \pm 0.016$ & $0.014 \pm 0.017$ & $0.095 \pm 0.017$ \\
\hline & & -2.5 & $0.014 \pm 0.015$ & $-0.024 \pm 0.016$ & $0.111 \pm 0.017$ \\
\hline & \multirow[t]{2}{*}{+2500} & +2.5 & $0.012 \pm 0.021$ & $0.025 \pm 0.023$ & $0.071 \pm 0.024$ \\
\hline & & -2.5 & $0.001 \pm 0.019$ & $-0.049 \pm 0.022$ & $0.111 \pm 0.022$ \\
\hline \multirow[t]{4}{*}{-1700} & \multirow[t]{2}{*}{-2500} & +2.5 & $0.012 \pm 0.023$ & $-0.008 \pm 0.024$ & $0.156 \pm 0.022$ \\
\hline & & -2.5 & $0.041 \pm 0.020$ & $0.026 \pm 0.020$ & $0.123 \pm 0.020$ \\
\hline & \multirow[t]{2}{*}{+2500} & +2.5 & $0.018 \pm 0.027$ & $0.038 \pm 0.029$ & $0.088 \pm 0.028$ \\
\hline & & -2.5 & $0.019 \pm 0.026$ & $-0.011 \pm 0.028$ & $0.146 \pm 0.025$ \\
\hline \multirow[t]{4}{*}{-2500} & \multirow[t]{2}{*}{-2500} & +2.5 & $0.052 \pm 0.042$ & $-0.040 \pm 0.045$ & $0.132 \pm 0.045$ \\
\hline & & -2.5 & $0.048 \pm 0.040$ & $0.002 \pm 0.041$ & $0.080 \pm 0.042$ \\
\hline & \multirow[t]{2}{*}{+2500} & +2.5 & $0.013 \pm 0.057$ & $0.040 \pm 0.061$ & $0.024 \pm 0.059$ \\
\hline & & -2.5 & $0.058 \pm 0.047$ & $0.027 \pm 0.051$ & $0.160 \pm 0.040$ \\
\hline \multirow[t]{4}{*}{-3300} & \multirow[t]{2}{*}{-2500} & +2.5 & $0.052 \pm 0.040$ & $-0.056 \pm 0.045$ & $0.064 \pm 0.042$ \\
\hline & & -2.5 & $-0.021 \pm 0.032$ & $-0.029 \pm 0.033$ & $0.153 \pm 0.029$ \\
\hline & \multirow[t]{2}{*}{+2500} & +2.5 & $0.010 \pm 0.055$ & $-0.051 \pm 0.058$ & $0.154 \pm 0.047$ \\
\hline & & -2.5 & $0.007 \pm 0.054$ & $-0.108 \pm 0.056$ & $0.135 \pm 0.049$ \\
\hline
\end{tabular}

Table 5.1: Phase 1. $\Omega^{-}$asymmetry results from the Hybrid Monte Carlo. These were used in Equations 5.25 and 5.26. 


\begin{tabular}{|c|c|c|c|c|c|}
\hline $\begin{array}{c}\text { Hyperon } \\
\text { Magnet } \\
(\text { Amps })\end{array}$ & $\begin{array}{c}\text { BM109 } \\
\text { Current } \\
(\text { Amps })\end{array}$ & $\begin{array}{c}\text { A.vg. } \Omega^{-} \\
\text {Momentum } \\
(\mathrm{GeV} / \mathrm{c})\end{array}$ & $P_{x}$ & $P_{y}$ & $P_{z}$ \\
\hline \multirow{2}{*}{500} & -2500 & 335. & $0.002 \pm 0.034$ & $0.019 \pm 0.037$ & $0.032 \pm 0.040$ \\
\cline { 2 - 6 } & +2500 & 320. & $-0.087 \pm 0.035$ & $-0.021 \pm 0.038$ & $0.026 \pm 0.040$ \\
\hline \multirow{2}{*}{-1000} & -2500 & 394. & $-0.030 \pm 0.017$ & $0.029 \pm 0.018$ & $-0.012 \pm 0.018$ \\
\cline { 2 - 6 } & +2500 & 384. & $0.008 \pm 0.022$ & $0.057 \pm 0.027$ & $-0.031 \pm 0.025$ \\
\hline \multirow{2}{*}{-1700} & -2500 & 433. & $-0.023 \pm 0.023$ & $-0.026 \pm 0.025$ & $0.026 \pm 0.023$ \\
\cline { 2 - 6 } & +2500 & 423. & $-0.000 \pm 0.029$ & $0.038 \pm 0.031$ & $-0.046 \pm 0.029$ \\
\hline \multirow{2}{*}{2500} & -2500 & 459. & $0.004 \pm 0.045$ & $-0.032 \pm 0.048$ & $0.040 \pm 0.048$ \\
\cline { 2 - 6 } & +2500 & 451. & $-0.035 \pm 0.058$ & $0.010 \pm 0.062$ & $-0.106 \pm 0.055$ \\
\hline \multirow{2}{*}{-3300} & -2500 & 479. & $0.057 \pm 0.040$ & $-0.021 \pm 0.043$ & $-0.069 \pm 0.040$ \\
\cline { 2 - 6 } & +2500 & 470. & $0.028 \pm 0.060$ & $0.044 \pm 0.063$ & $0.015 \pm 0.053$ \\
\hline
\end{tabular}

Table 5.2: Phase 1. Polarization results at $\pm 2.5 \mathrm{mR}$ targeting angle for the $\Omega^{-}$data from the Hybrid Monte Carlo. It is assumed that $\gamma_{\Omega^{-}}=1$. Otherwise, the polarizations and uncertainties should be scaled by $-5 / 3$.

\begin{tabular}{|c|c|c|c|c|c|}
\hline $\begin{array}{c}\text { Hyperon } \\
\text { Magnet } \\
\text { (Amps) }\end{array}$ & $\begin{array}{c}\text { BM109 } \\
\text { Current } \\
(\text { Amps })\end{array}$ & $\begin{array}{c}\text { Avg. } \Omega^{-} \\
\text {Momentum } \\
(\mathrm{GeV} / \mathrm{c})\end{array}$ & $B_{x}$ & $B_{y}$ & $B_{z}$ \\
\hline \multirow{2}{*}{500} & -2500 & 335. & $-0.008 \pm 0.022$ & $0.022 \pm 0.024$ & $0.096 \pm 0.026$ \\
\cline { 2 - 6 } & +2500 & 325. & $0.029 \pm 0.022$ & $0.007 \pm 0.024$ & $0.162 \pm 0.026$ \\
\hline \multirow{2}{*}{-1000} & -2500 & 394. & $-0.005 \pm 0.011$ & $-0.005 \pm 0.012$ & $0.103 \pm 0.012$ \\
\cline { 2 - 7 } & +2500 & 384. & $0.006 \pm .014$ & $-0.012 \pm 0.017$ & $0.091 \pm 0.016$ \\
\hline \multirow{2}{*}{-1700} & -2500 & 433. & $0.026 \pm 0.015$ & $0.009 \pm 0.016$ & $0.139 \pm 0.015$ \\
\cline { 2 - 7 } & +2500 & 423. & $0.019 \pm 0.019$ & $0.013 \pm 0.020$ & $0.117 \pm 0.019$ \\
\hline \multirow{2}{*}{-2500} & -2500 & 459. & $0.050 \pm 0.029$ & $-0.019 \pm 0.031$ & $0.106 \pm 0.031$ \\
\cline { 2 - 6 } & +2500 & 451. & $0.035 \pm 0.037$ & $0.033 \pm 0.040$ & $0.092 \pm 0.035$ \\
\hline \multirow{2}{*}{-3300} & -2500 & 479. & $0.015 \pm 0.026$ & $-0.042 \pm 0.028$ & $0.108 \pm 0.026$ \\
\cline { 2 - 6 } & +2500 & 470. & $0.008 \pm 0.039$ & $-0.080 \pm 0.040$ & $0.144 \pm 0.034$ \\
\hline
\end{tabular}

Table 5.3: Phase 1 . Bias results at $\pm 2.5 \mathrm{mR}$ targeting angle for the $\Omega^{-}$data from the Hybrid Monte Carlo. 


\begin{tabular}{|c|c|c|c|c|c|c|c|}
\hline $\begin{array}{c}\text { Hyperon } \\
\text { Magnet } \\
\text { (Amps) }\end{array}$ & $\begin{array}{l}\text { BM109 } \\
\text { Current } \\
\text { (Amps) }\end{array}$ & $\begin{array}{l}\text { Tgting } \\
\text { Angle } \\
(\mathrm{mR}) \\
\end{array}$ & $\begin{array}{c}\text { Avg. } \Omega^{-} \\
\text {Momentum } \\
(\mathrm{GeV} / \mathrm{c})\end{array}$ & $\begin{array}{c}\text { Final } \\
\text { Event } \\
\text { Sample }\end{array}$ & $\chi_{x}^{2}$ & $\chi_{y}^{2}$ & $\chi_{z}^{2}$ \\
\hline \multirow[t]{4}{*}{-500} & \multirow[t]{2}{*}{-2500} & +2.5 & 334. & 3451 & 44.2 & 31.4 & 46.6 \\
\hline & & -2.5 & 335. & 3580 & 29.0 & 60.4 & 33.4 \\
\hline & \multirow[t]{2}{*}{+2500} & +2.5 & 320. & 3827 & 42.6 & 81.1 & $\overline{57.7}$ \\
\hline & & -2.5 & 320. & 3062 & 36.6 & 57.5 & 58.7 \\
\hline \multirow[t]{4}{*}{-1000} & \multirow[t]{2}{*}{-2500} & +2.5 & 394. & 12749 & 59.5 & 178. & 139. \\
\hline & & -2.5 & 394. & 13262 & 47.8 & 135. & 119. \\
\hline & \multirow[t]{2}{*}{+2500} & +2.5 & 384. & 6925 & 65.7 & 71.3 & 80.5 \\
\hline & & -2.5 & 383. & 7938 & 42.7 & 110. & 58.1 \\
\hline \multirow[t]{4}{*}{-1700} & \multirow[t]{2}{*}{-2500} & +2.5 & 434. & 5934 & 56.9 & 99.2 & 108. \\
\hline & & -2.5 & 432. & 7831 & 59.9 & 99.4 & 107. \\
\hline & \multirow[t]{2}{*}{+2500} & +2.5 & 423. & 3998 & 34.7 & 76.3 & 53.6 \\
\hline & & -2.5 & 423. & 4306 & 39.0 & 57.1 & 89.3 \\
\hline \multirow[t]{4}{*}{-2500} & \multirow[t]{2}{*}{-2500} & +2.5 & 462. & 1689 & 33.0 & 50.2 & 28.3 \\
\hline & & -2.5 & 457. & 1879 & 32.4 & 50.6 & 46.3 \\
\hline & \multirow[t]{2}{*}{+2500} & +2.5 & 452. & 862 & 18.0 & 33.5 & 32.7 \\
\hline & & -2.5 & 451. & 1275 & 25.4 & 64.4 & 24.0 \\
\hline \multirow[t]{4}{*}{-3300} & \multirow[t]{2}{*}{-2500} & +2.5 & 479. & 1859 & 28.6 & 46.2 & 37.6 \\
\hline & & -2.5 & 479. & 3099 & 30.9 & 36.8 & 67.0 \\
\hline & \multirow[t]{2}{*}{+2500} & +2.5 & 469. & 1008 & 20.9 & 27.2 & 42.4 \\
\hline & & -2.5 & 471. & 1079 & 41.6 & 29.6 & 29.3 \\
\hline
\end{tabular}

Table 5.4: Phase 1. Statistics of the $\Omega^{-}$polarization results from the Hybrid Monte Carlo. 
The horizontal targeting angle data at $\pm 1.3 \mathrm{mR}$ data was analyzed with the Hybrid Monte Carlo. The horizontal targeting angle data was produced by rotating the dipole magnets in PC3 by 90 degrees and setting up a targeting angle in the $\hat{z} \times \hat{x}$ plane. Any polarization would be parallel to $\hat{y}=\hat{z} \times \hat{x}$. It would not precess in the Hyperon Magnet because the magnetic field is in the $-\hat{y}$ direction. Because the sample was small, all of the events at a given targeting angle and BM109 current were combined. The combined result was that the main component, $P_{y}=0.00 \pm 0.05$. The parity-violating components were $P_{x}=-0.03 \pm 0.05$ and $P_{z}=0.09 \pm 0.05$. The average momentum was $450 \mathrm{GeV} / \mathrm{c}$ from a total of 3089 events.

The asymmetry results from the Hybrid Monte Carlo for each targeting angle at the five magnetic fields were combined, along with the value of the $\Omega^{-}$g-factor measured in Phase 2, in a "grand" $\chi^{2}$ fit. This method of combining the $\mathrm{x}$ and $\mathrm{z}$ components of the polarization supplies another measurement of the polarization at the target and the bias for each component. The $\chi^{2}$ expression is:

$$
\chi^{2}=\sum_{i} \sum_{j} \sum_{k}\left\{\frac{\left(\alpha_{\Lambda} P_{x_{i j k}} \pm \alpha_{\Lambda} P_{i} \cos \theta_{i}-B_{x_{i k}}\right)^{2}}{\left(\delta\left[\alpha_{\Lambda} P_{x_{i j k}}\right]\right)^{2}}+\frac{\left(\alpha_{\Lambda} P_{z_{i j k}} \pm \alpha_{\Lambda} P_{i} \sin \theta_{i}-B_{z_{i k}}\right)^{2}}{\left(\delta\left[\alpha_{\Lambda} P_{z_{i j k}}\right]\right)^{2}}\right\}
$$

where the sum over $i$ is for each Hyperon Magnet current, $\theta_{i}$ is the expected precession due at the ith value of the Hyperon Magnet current, the sum over $j$ is for \pm targeting angle, and the sum over $k$ is for the two BM109 currents. $\alpha_{\Lambda} P_{x_{i j k}}$ and $\alpha_{\Lambda} P_{y_{i j k}}$ are the asymmetries measured by the Hybrid Monte Carlo. The \pm is positive for positive targeting angle and negative for negative targeting angle. Again the two BM109 currents are treated independently because the biases may be different. The parameters which come from the fit are: five polarizations, $P_{i}$, one at each Hyperon Magnet current; and ten values of each component, $B_{x_{i k}}$ and $B_{z_{i k}}$, of the bias. Therefore, there are 40 input parameters, 25 fitted parameters, and 15 degrees of freedom.

The following summarizes the results of the fit. Table 5.5 presents the polarization results as a function of $\Omega^{--}$momentum. The variation of the polarization as the g-factor is changed by one sigma is also shown. These polarizations are plotted in Figure 5.4. 


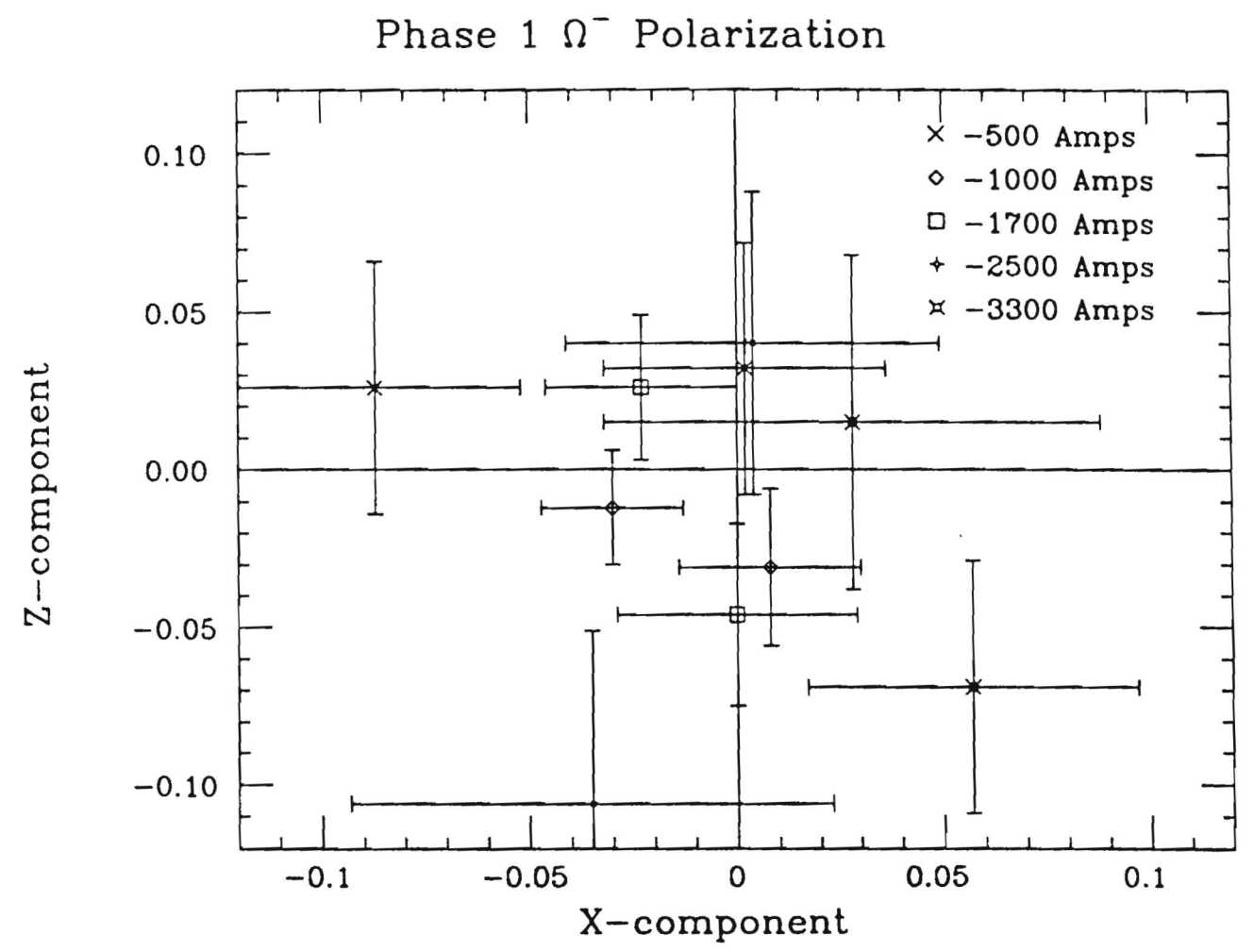

Figure 5.3: Phase $1 \Omega^{-}$Polarization components. Plotted are $P_{x}$ vs. $P_{z}$ for the 5 fields. These are from Table 5.2. $\gamma_{n}$ is assumed +1 for this plot. 


\begin{tabular}{|c|c|c|c|c|}
\hline $\begin{array}{c}\text { Hyperon } \\
\text { Magnet } \\
(\text { Amps })\end{array}$ & $\begin{array}{c}\text { Avg. } \Omega^{-} \\
\text {Momentum } \\
(\mathrm{GeV} / \mathrm{c})\end{array}$ & $\begin{array}{c}\text { Polarization } \\
\text { assuming } \\
\mathrm{g}=2.40\end{array}$ & $\begin{array}{c}\delta P \\
\text { assuming } \\
\mathrm{g}=2.21\end{array}$ & $\begin{array}{c}\delta P \\
\text { assuming } \\
\mathrm{g}=2.59\end{array}$ \\
\hline-500 & 328 & $-0.025 \pm 0.025$ & +0.009 & -0.010 \\
\hline-1000 & 390 & $-0.022 \pm 0.014$ & -0.003 & +0.000 \\
\hline-1700 & 429 & $-0.011 \pm 0.018$ & +0.003 & -0.003 \\
\hline-2500 & 456 & $-0.024 \pm 0.036$ & -0.005 & +0.001 \\
\hline-3300 & 476 & $-0.008 \pm 0.032$ & -0.024 & +0.021 \\
\hline$\chi \chi 2 /$ d.f. & & $22.3 / 15$ & $21.7 / 15$ & $22.5 / 15$ \\
\hline
\end{tabular}

Table 5.5: Phase $1 \Omega^{-}$grand fit results. The polarization is presented $\gamma$ is +1 . The polarization and error bar are multiplied by $-5 / 3$ if $\gamma$ is -1 . The last two columns are the change in polarization when $g$ is changed by $\pm 1 \sigma$ (e.g. $P_{g=2.40}-P_{g=2.59}$ ).

Table 5.6 presents the the components of the bias. The components of the bias are shown in Figures 5.5 and 5.6. The $\chi^{2}$ of the fit was 22.3 for 15 degrees of freedom. In the presentation of these results, $\gamma_{\Omega}$ was assumed to be +1 . If $\gamma_{\Omega}$ is -1 , then the polarizations, biases and error bars are to be multiplied by $-5 / 3$.

The weighted average of the polarization results is $-0.018 \pm 0.009$. Even though some of the individual measurements varied slightly with the $\mathrm{g}$-factor, this average did not. This polarization and momentum dependence is most like the unpolarized antiLambdas in Figure 2.1. The weighted average $P_{y}$ of the combined sample from Table 5.2 is $1.5 \pm 1.0 \%$.

When the grand fit was applied to the two BM109 current samples independently, the weighted average of the -2500 Amp sample was $-0.010 \pm 0.012$ and the weighted average of the +2500 Amp sample was $-0.031 \pm 0.015$. 


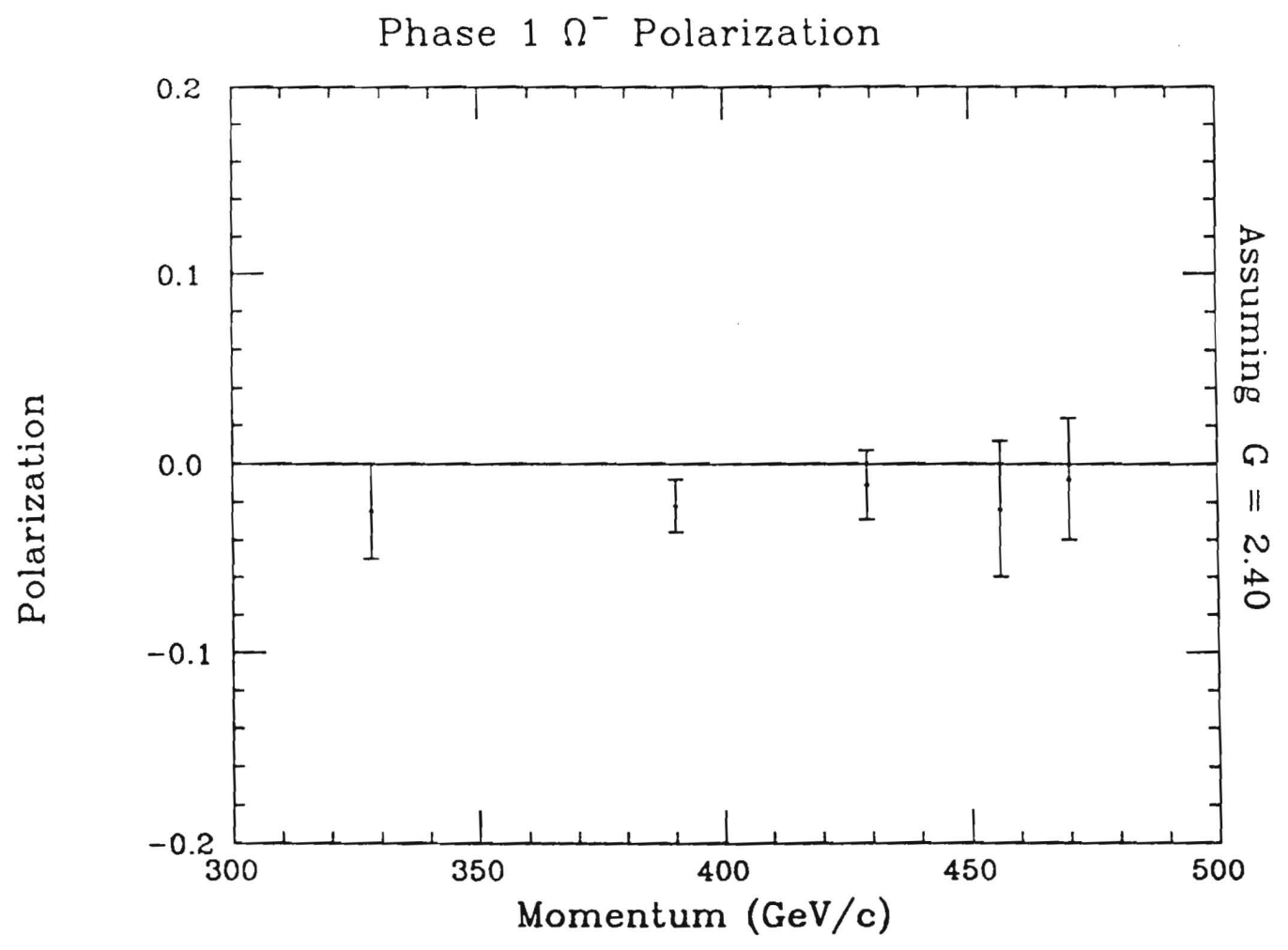

Figure 5.4: Phase 1 Polarization. These are the results of the grand fit assuming the g-factor is 2.40 as measured in Phase 2. The results are presented assuming $\gamma=+1$. If $\gamma_{\Omega}$ is -1 , scale the polarization and uncertainties by $-5 / 3$. 
Phase $1 \Omega^{-}$Bias

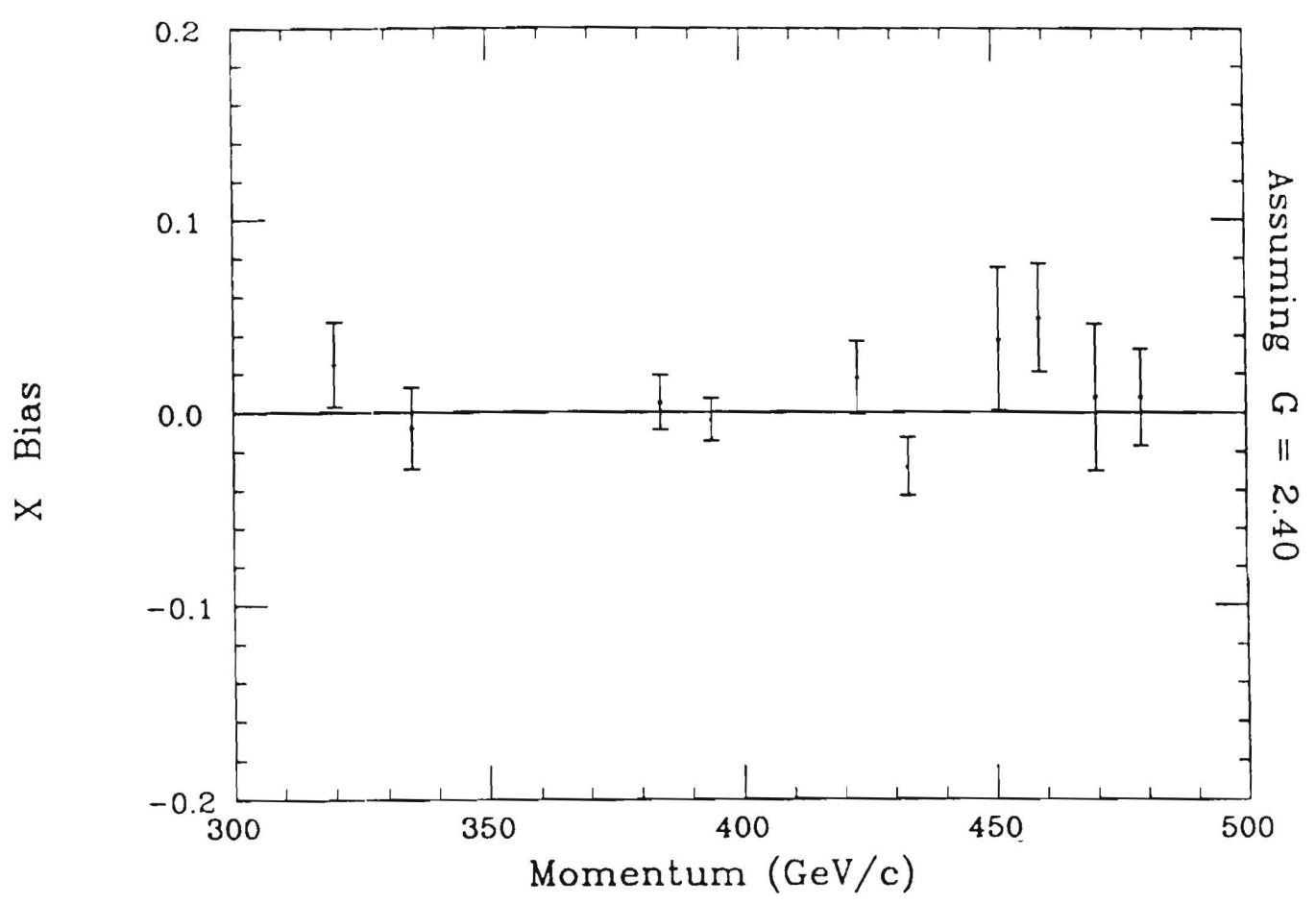

Figure 5.5: Phase $1 \hat{x}$ Bias. These are the results of the grand fit assuming the g-factor is 2.40 as measured in Phase 2.

\begin{tabular}{|c|c|c|c|c|}
\hline $\begin{array}{c}\text { Hyperon } \\
\text { Magnet } \\
\text { (Amps) }\end{array}$ & $\begin{array}{c}\text { Avg. } \Omega^{-} \\
\text {Momentum } \\
(\text { GeV/c) }\end{array}$ & $\begin{array}{c}\text { BM109 } \\
\text { Current } \\
(\text { Amps })\end{array}$ & $B_{x}$ & $B_{z}$ \\
\hline \multirow{2}{*}{500} & 335 & -2500 & $-0.008 \pm 0.021$ & $0.098 \pm 0.026$ \\
\cline { 2 - 5 } & 320 & +2500 & $+0.025 \pm 0.022$ & $0.165 \pm 0.026$ \\
\hline \multirow{2}{*}{-1000} & 394 & -2500 & $-0.004 \pm 0.011$ & $0.105 \pm 0.012$ \\
\cline { 2 - 5 } & 384 & +2500 & $+0.005 \pm 0.014$ & $0.091 \pm 0.016$ \\
\hline \multirow{2}{*}{-1700} & 433 & -2500 & $+0.028 \pm 0.015$ & $0.137 \pm 0.015$ \\
\cline { 2 - 5 } & 423 & +2500 & $+0.018 \pm 0.019$ & $0.120 \pm 0.019$ \\
\hline \multirow{2}{*}{-2500} & 459 & -2500 & $+0.049 \pm 0.028$ & $0.103 \pm 0.031$ \\
\cline { 2 - 5 } & 451 & +2500 & $+0.038 \pm 0.037$ & $0.113 \pm 0.033$ \\
\hline-3300 & 479 & -2500 & $+0.008 \pm 0.025$ & $0.124 \pm 0.024$ \\
\cline { 2 - 5 } & 470 & +2500 & $+0.008 \pm 0.038$ & $0.145 \pm 0.033$ \\
\hline
\end{tabular}

Table 5.6: Phase $1 \Omega^{-}$grand fit bias results. 


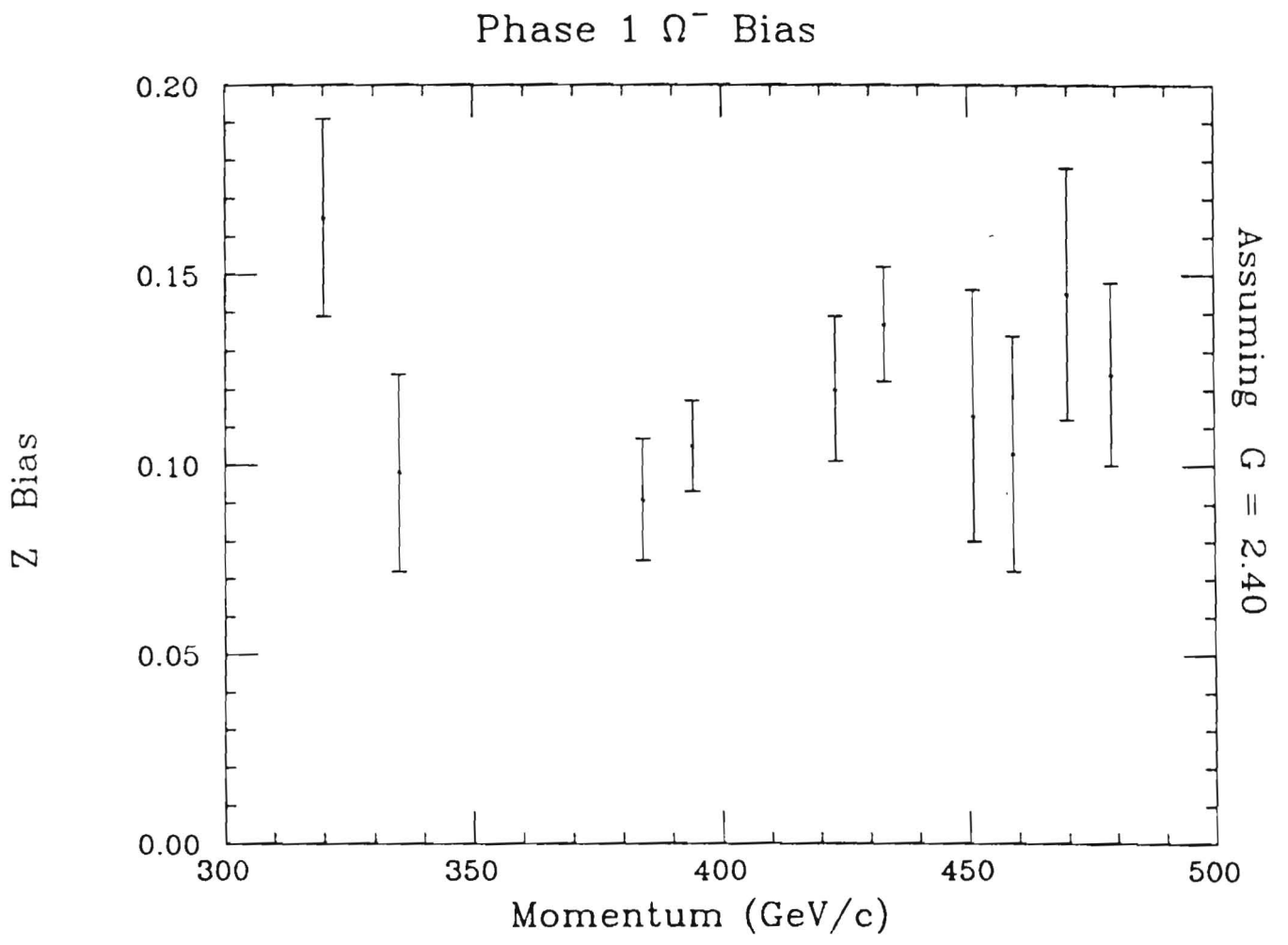

Figure 5.6: Phase $1 \hat{z}$ Bias. These are the results of the grand fit assuming the g-factor is 2.40 as measured in Phase 2. 


\subsection{Phase 1 Cascade Minus Polarization Results}

The polarization of a small sample of the Phase $1 \Xi^{-}$events was determined for comparison with the polarization of the Phase $1 \Omega^{-}$s. The results of analysis of $\sim 1$ to 2 data tapes per targeting angle for each of the 5 Hyperon Magnet currents are presented. A complete analysis of the Phase $1 \Xi^{-s}$ is the subject of a more detailed study [47].

In order to improve the $\chi^{2}$ of the Hybrid Monte Carlo polarization fits and reduce the $\mathrm{z}$ bias, a "separation cut" was used to simulate the effects of the reconstruction program. The separation, $\Delta S$, was defined as

$$
\Delta S=\sum_{i=x \text { and } y} \sum_{j=C i \text { and } C 6}\left(\text { proton position }_{i j}-\text { pion position }_{i j}\right)
$$

That is, the separation is the sum of difference between the position of the proton and the pion from the $\Lambda$ decay in the $\mathrm{x}$ view of $\mathrm{C} 4$, the $\mathrm{x}$ view of $\mathrm{C} 6$, the $\mathrm{y}$ view of $\mathrm{C} 4$ and the $y$ view of 66 . When the proton went nearly backwards in the $\Lambda$ rest frame, the proton track was close to the pion track in the upstream chambers. The tracking program had smaller acceptance for these events. The "separation cut" was the Hybrid Monte Carlo method of characterizing the tracking program's acceptance. The bias and $\chi^{2}$ of the polarization results were scanned as a function of separation cut from $\Delta S=0$. to 6 . $\mathrm{cm}$. The best $\chi^{2}$ and bias results were found by taking a separation cut of separation $=4.0 \mathrm{~cm}$ and removing events with $\cos \theta_{z}<-0.80$. Also, minimum $\mathrm{x}$ and y separations of $0.6 \mathrm{~cm} \mathrm{0.4:} \mathrm{cm}$ were required. The $\chi^{2}$ of the polarization fits improved by a factor of $\approx 10$. The $z$ bias was reduced. The cost was $\approx 20 \%$ of the data. Except for this separation cut, the same aperture cuts were used in analyzing the $\Xi^{-}$as were used when analyzing the $\Omega^{-}$data. The polarization and it's components did not change significantly depending on the final choice of these cuts.

The Hybrid Monte Carlo asymmetry results are presented in Table 5.7. The components of the polarization and bias determined from combining the two targeting angles are presented in Tables 5.3 and 5.9. Table 5.10 shows the statistics of the polarization fits in the Hybrid Monte Carlo. Finally, Table 5.11 contains magnitude and direction 


\begin{tabular}{|c|c|c|c|c|c|}
\hline $\begin{array}{c}\text { Hyperon } \\
\text { Magnet } \\
\text { (Amps) }\end{array}$ & $\begin{array}{c}\text { BM109 } \\
\text { Current } \\
\text { (Amps) }\end{array}$ & $\begin{array}{c}\text { Tgting } \\
\text { Angle } \\
(\mathrm{mR})\end{array}$ & $\begin{array}{c}\text { Asymmetry } \\
\hat{x} \\
\text { Component }\end{array}$ & $\begin{array}{c}\text { Asymmetry } \\
\hat{y} \\
\text { Component }\end{array}$ & $\begin{array}{c}\text { Asymmetry } \\
\hat{z} \\
\text { Component }\end{array}$ \\
\hline-500 & -2500 & +2.5 & $-0.049 \pm 0.006$ & $-0.013 \pm 0.006$ & $0.026 \pm 0.008$ \\
\cline { 3 - 6 } & & -2.5 & $0.068 \pm 0.006$ & $-0.001 \pm 0.006$ & $0.007 \pm 0.008$ \\
\hline-1000 & -2500 & +2.5 & $-0.076 \pm 0.006$ & $-0.016 \pm 0.006$ & $0.009 \pm 0.008$ \\
\cline { 3 - 6 } & & -2.5 & $0.065 \pm 0.007$ & $0.013 \pm 0.007$ & $-0.023 \pm 0.010$ \\
\hline-1700 & -2500 & +2.5 & $-0.066 \pm 0.006$ & $-0.021 \pm 0.006$ & $0.029 \pm 0.007$ \\
\hline & & -2.5 & $0.058 \pm 0.006$ & $-0.002 \pm 0.006$ & $-0.042 \pm 0.008$ \\
\hline-2500 & -2500 & +2.5 & $-0.070 \pm 0.007$ & $-0.027 \pm 0.007$ & $0.028 \pm 0.009$ \\
\cline { 3 - 6 } & & -2.5 & $0.048 \pm 0.006$ & $0.004 \pm 0.006$ & $-0.038 \pm 0.008$ \\
\hline-3300 & -2500 & +2.5 & $-0.068 \pm 0.010$ & $-0.009 \pm 0.009$ & $0.011 \pm 0.012$ \\
\cline { 3 - 6 } & & -2.5 & $0.045 \pm 0.008$ & $0.002 \pm 0.007$ & $-0.035 \pm 0.010$ \\
\hline
\end{tabular}

Table 5.7: Phase 1. $\Xi^{-}$asymmetry results from the Hybrid Monte Carlo. These were used in Equations 5.25 and 5.26.

of the polarization at the target, before it precessed in the Hyperon Magnet. Figure 5.7 show $P_{x}$ vs. $P_{z}$ for the field integrals. Since $\gamma_{\Xi}$ is known, the results are reported as $\vec{P}_{\Xi-}$ rather than $\vec{P}_{\Lambda}$. 


\begin{tabular}{|c|c|c|c|c|c|}
\hline $\begin{array}{c}\text { Hyperon } \\
\text { Magnet } \\
(\text { Amps })\end{array}$ & $\begin{array}{c}\text { BM109 } \\
\text { Current } \\
(\text { Amps })\end{array}$ & $\begin{array}{c}\text { Avg. } \Xi^{-} \\
\text {Momentum } \\
(\mathrm{GeV} / \mathrm{c})\end{array}$ & $P_{x}$ & $P_{y}$ & $P_{z}$ \\
\hline-500 & -2500 & 330. & $-0.093 \pm 0.007$ & $-0.010 \pm 0.007$ & $0.017 \pm 0.010$ \\
\hline-1000 & -2500 & 393. & $-0.112 \pm 0.008$ & $-0.025 \pm 0.008$ & $0.027 \pm 0.011$ \\
\hline-1700 & -2500 & 433. & $-0.103 \pm 0.007$ & $-0.016 \pm 0.007$ & $0.059 \pm 0.009$ \\
\hline-2500 & -2500 & 464. & $-0.099 \pm 0.008$ & $-0.026 \pm 0.008$ & $0.055 \pm 0.010$ \\
\hline-3300 & -2500 & 485. & $-0.094 \pm 0.010$ & $-0.009 \pm 0.010$ & $0.039 \pm 0.013$ \\
\hline
\end{tabular}

Table 5.8: Phase 1. Polarization results at $\pm 2.5 \mathrm{mR}$ targeting angle for the $\Xi^{-}$data from the Hybrid Monte Carlo.

\begin{tabular}{|c|c|c|c|}
\hline $\begin{array}{c}\text { Hyperon } \\
\text { Magnet } \\
(\text { Amps })\end{array}$ & $\begin{array}{c}\text { Avg. } \Xi^{-} \\
\text {Momentum } \\
(\mathrm{GeV} / \mathrm{c})\end{array}$ & $\begin{array}{c}\text { Avg. } \\
\left|P_{t}\right| \\
(\mathrm{GeV} / \mathrm{c})\end{array}$ & Polarization \\
\hline-500 & 331. & 0.83 & $-0.095 \pm 0.007$ \\
\hline-1000 & 394. & 0.99 & $-0.121 \pm 0.008$ \\
\hline-1700 & 434. & 1.09 & $-0.119 \pm 0.008$ \\
\hline-2500 & 464. & 1.16 & $-0.113 \pm 0.008$ \\
\hline-3300 & 485. & 1.21 & $-0.102 \pm 0.011$ \\
\hline
\end{tabular}

Table 5.9: Phase 1. $\Xi^{-}$polarization results at $\pm 2.5 \mathrm{mR}$ targeting angle. These are the initial polarizations at the $\Xi^{-}$production target. They are calculated by taking $\left(P_{x}^{2}+P_{z}^{2}\right)^{1 / 2}$. 


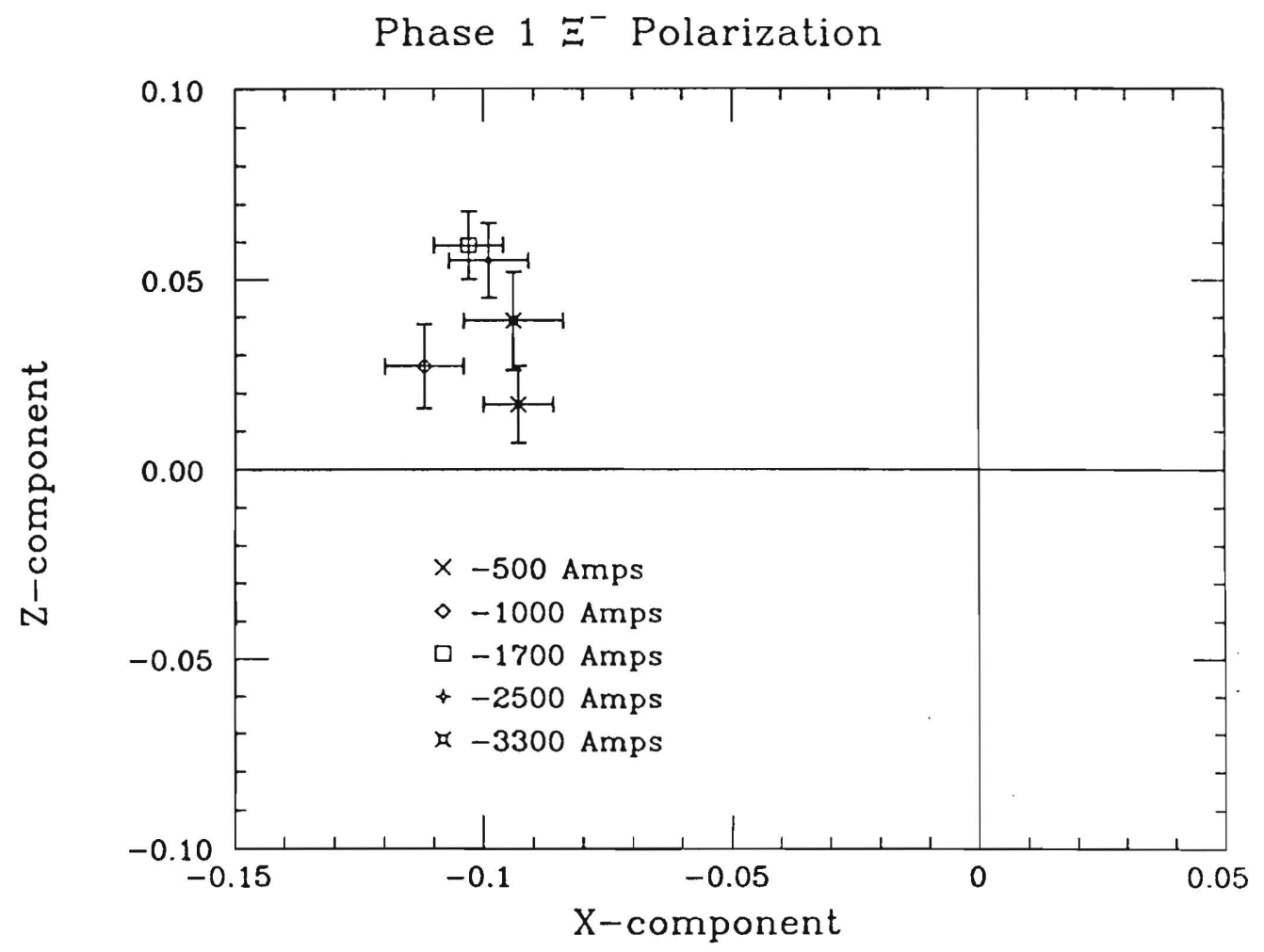

Figure 5.7: Phase $1 \Xi^{-}$Polarization components. Plotted are $P_{x}$ vs. $P_{z}$ for each of the 5 field integrals. 


\begin{tabular}{|c|c|c|c|c|c|}
\hline $\begin{array}{c}\text { Hyperon } \\
\text { Magnet } \\
\text { (Amps) }\end{array}$ & $\begin{array}{c}\text { BM109 } \\
\text { Current } \\
(\text { Amps })\end{array}$ & $\begin{array}{c}\text { Avg. } \Xi^{-} \\
\text {Momentum } \\
(\mathrm{GeV} / \mathrm{c})\end{array}$ & $B_{\boldsymbol{x}}$ & $B_{y}$ & $B_{\boldsymbol{z}}$ \\
\hline-500 & -2500 & $\mathbf{3 3 1 .}$ & $0.010 \pm 0.004$ & $-0.007 \pm 0.004$ & $0.016 \pm 0.006$ \\
\hline-1000 & -2500 & $\mathbf{3 9 4 .}$ & $-0.006 \pm 0.005$ & $-0.002 \pm 0.005$ & $-0.007 \pm 0.006$ \\
\hline-1700 & -2500 & 434. & $-0.004 \pm 0.004$ & $-0.012 \pm 0.004$ & $0.006 \pm 0.006$ \\
\hline-2500 & -2500 & 464. & $-0.011 \pm 0.005$ & $-0.011 \pm 0.005$ & $-0.005 \pm 0.006$ \\
\hline-3300 & -2500 & 485. & $-0.012 \pm 0.006$ & $-0.004 \pm 0.006$ & $-0.012 \pm 0.008$ \\
\hline
\end{tabular}

Table 5.10: Phase 1. Bias results at $\pm 2.5 \mathrm{mR}$ targeting angle for the $\Xi^{-}$data from the Hybrid Monte Carlo. 


\begin{tabular}{|c|c|c|c|c|c|c|c|}
\hline $\begin{array}{c}\text { Hyperon } \\
\text { Magnet } \\
\text { (Amps) }\end{array}$ & $\begin{array}{c}\text { BM109 } \\
\text { Current } \\
(\text { Amps })\end{array}$ & $\begin{array}{c}\text { Tgting } \\
\text { Angle } \\
(\mathrm{mR})\end{array}$ & $\begin{array}{c}\text { Avg. } \Xi^{-} \\
\text {Momentum } \\
(\mathrm{GeV} / \mathrm{c})\end{array}$ & $\begin{array}{c}\text { Final } \\
\text { Event } \\
\text { Sample }\end{array}$ & $\chi_{x}^{2}$ & $\chi_{y}^{2}$ & $\chi_{z}^{2}$ \\
\hline \multirow{2}{*}{500} & -2500 & +2.5 & 331. & 78854 & 39.1 & 49.1 & 104. \\
\hline & & -2.5 & 330. & 84188 & 35.9 & 87.4 & 91.1 \\
\hline \multirow{2}{*}{-1000} & \multirow{2}{*}{-2500} & +2.5 & 394. & 77993 & 61.4 & 55.3 & 103. \\
\cline { 3 - 8 } & & -2.5 & 391. & 49935 & 53.3 & 41.1 & 63.3 \\
\hline \multirow{2}{*}{-1700} & \multirow{2}{*}{-2500} & +2.5 & 435. & 89409 & 95.0 & 41.2 & 180. \\
\cline { 3 - 8 } & & -2.5 & 432. & 69109 & 91.6 & 61.9 & 126. \\
\hline \multirow{2}{*}{2500} & \multirow{2}{*}{-2500} & +2.5 & 467. & 63556 & 84.0 & 79.3 & 189. \\
\cline { 3 - 8 } & & -2.5 & 461. & 68146 & 70.0 & 56.3 & 120. \\
\hline \multirow{2}{*}{-3300} & \multirow{2}{*}{-2500} & +2.5 & 485. & 31899 & 57.4 & 52.7 & 70.2 \\
\cline { 3 - 8 } & & -2.5 & 485. & 50554 & 82.1 & 42.6 & 80.9 \\
\hline
\end{tabular}

Table 5.11: Phase 1. Statistics of the $\Xi^{-}$polarization results from the Hybrid Monte Carlo. 
The average $\hat{x}$ polarization at the target is $-0.109 \pm 0.004$. This is in contrast to the $-0.018 \pm 0.009$ Phase $1 \Omega^{-}$polarization.

There is a small $(-0.0173 \pm 0.0035)$ component in the $\hat{y}$ direction. This is in the nominal production plane and was expected to be zero. It is most likely that there was some small $\hat{x}$ component in the targeting angle of the runs analyzed which would vanish when averaged over many tapes taken far apart in time. This is to be taken as a systematic uncertainty in the overall $\Xi^{-}$polarization at the target. Because it is parallel to the Hyperon Magnet field, it does not precess into the $\hat{x}$ or $\hat{z}$ direction and therefore does not affect a nagnetic moment measurement made from this data.

\subsection{Phase 2 Omega Minus Polarization Results}

The $\Omega^{-}$polarization was also measured in the same way as for Phase 1. Unlike Phase 1, a clear $\Omega^{-}$polarization signal was discovered. Only the -450 amp sample has conclusive statistics. The -1000 amp sample supports the polarization. The other conditions do not provide enough $\Omega^{-}$s for polarization analysis. The polarization components are shown in Figure 5.8 as functions of targeting angle and Hyperon Magnet field. Table 5.12 shows the polarization results from the combined $\pm 2 \mathrm{mR}$ targeting angle data. $P_{x}$ vs. $P_{z}$ is plotted in Figure 5.9. Table 5.13 shows the direction and magnitude of the polarization at the $\Omega^{-}$production target assuming $\gamma_{\Omega^{-}}=1$. Table 5.14 shows the bias calculated from the $\pm 2 \mathrm{mR}$ data. Table 5.15 shows the $0 \mathrm{mR}$ polarization results. These provide another measure of the bias since the $0 \mathrm{mR}$ data should be unpolarized. Table 5.16 shows the statistics of the polarization fits in the Hybrid Monte Carlo. Finally, the $\Omega^{-}$and $\Xi^{-}$polarizations at the production target are plotted as a function of momentum in Figure 5.10. The results are that the $\Omega^{-} \mathrm{s}$, like the $\Xi^{-} \mathrm{s}$, are produced polarized by spin-transfer from the polarized neutral beam. The average polarization is $-0.069 \pm 0.022$. 

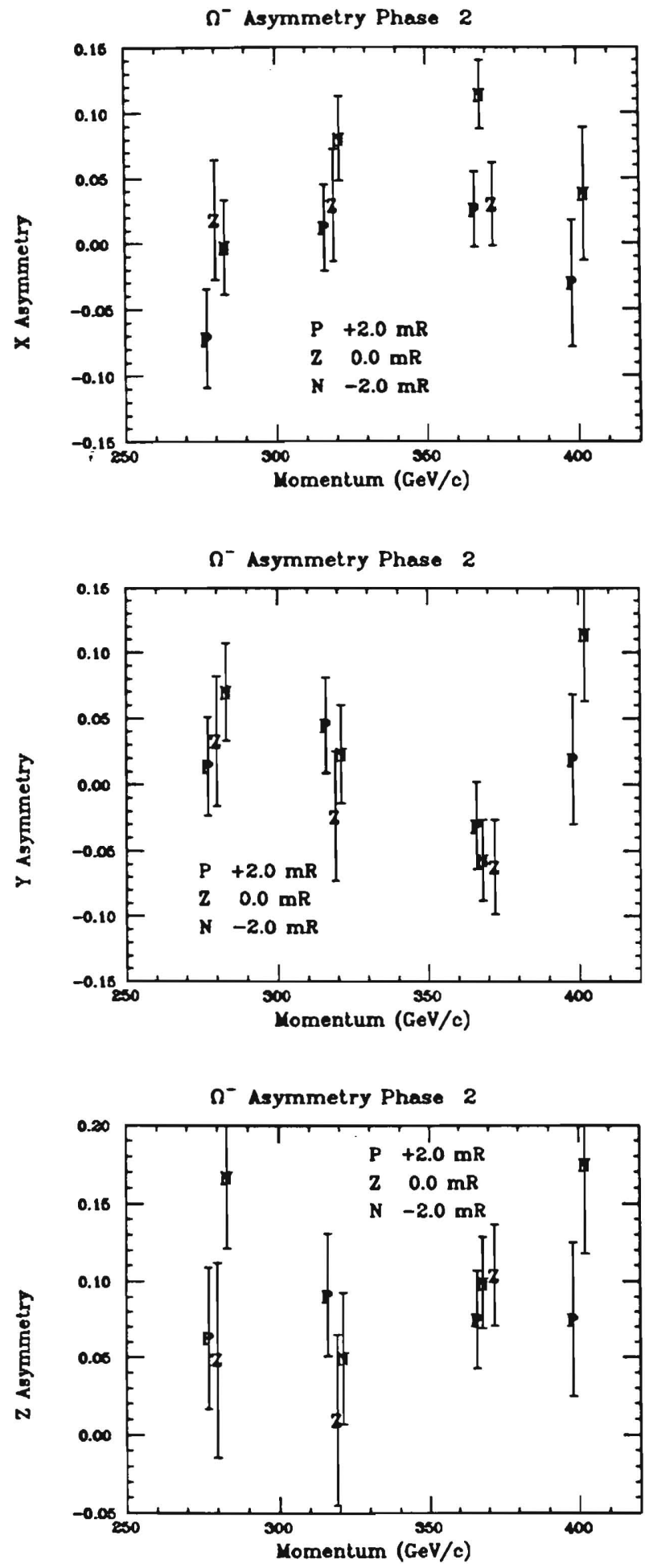

Figure 5.8: $\Omega^{-}$asymmetry components from Phase 2. The 3 lower momentum points are the $-450 \mathrm{Amp}$ sample. The highest momentum point is the $-1000 \mathrm{amp}$ sample. The targeting angle is indicated with a letter symbol. 


\begin{tabular}{|c|c|c|c|c|c|}
\hline $\begin{array}{c}\text { Hyp. } \\
\text { Magnet } \\
\text { (Amps) }\end{array}$ & $\begin{array}{l}\text { Tgt. } \\
\text { Angle } \\
(\mathrm{mR})\end{array}$ & $\begin{array}{c}\Omega^{--} \\
\text {Momentum } \\
(\mathrm{GeV} / \mathrm{c})\end{array}$ & $\begin{array}{c}\text { Asymmetry } \\
\hat{x} \\
\text { Component }\end{array}$ & $\begin{array}{c}\text { Asymmetry } \\
\hat{y} \\
\text { Component }\end{array}$ & $\begin{array}{c}\text { Asymmetry } \\
\hat{z} \\
\text { Component }\end{array}$ \\
\hline \multirow[t]{8}{*}{-450} & \multirow[t]{4}{*}{+2.0} & 280 & $-0.072 \pm 0.037$ & $0.014 \pm 0.037$ & $0.063 \pm 0.046$ \\
\hline & & 319. & $0.012 \pm 0.033$ & $0.045 \pm 0.036$ & $0.091 \pm 0.040$ \\
\hline & & 368. & $0.026 \pm 0.029$ & $-0.031 \pm 0.033$ & $0.075 \pm 0.032$ \\
\hline & & 323.(avg.) & $-0.006 \pm 0.019$ & $0.009 \pm 0.020$ & $0.082 \pm 0.022$ \\
\hline & \multirow[t]{4}{*}{-2.0} & 280. & $-0.003 \pm 0.036$ & $0.070 \pm 0.037$ & $0.167 \pm 0.046$ \\
\hline & & 319. & $0.080 \pm 0.032$ & $0.023 \pm 0.037$ & $0.050 \pm 0.043$ \\
\hline & & 368. & $0.114 \pm 0.026$ & $-0.057 \pm 0.031$ & $0.099 \pm 0.030$ \\
\hline & & 325.(avg.) & $0.071 \pm 0.018$ & $0.004 \pm 0.020$ & $0.103 \pm 0.022$ \\
\hline \multirow[t]{2}{*}{-1000} & +2.0 & 400. (avg.) & $-0.030 \pm 0.048$ & $0.019 \pm 0.049$ & $0.075 \pm 0.050$ \\
\hline & -2.0 & 40 (avg.) & $0.038 \pm 0.051$ & $0.114 \pm 0.051$ & $0.175 \pm 0.057$ \\
\hline
\end{tabular}

Table 5.12: Phase 2. $\Omega^{-}$asymmetry results from the Hybrid Monte Carlo. These were used in Equations 5.25 and 5.26. The results from $0 \mathrm{mR}$ are given in Table 5.16. 


\begin{tabular}{|c|c|c|c|c|}
\hline $\begin{array}{c}\text { Hyperon } \\
\text { Magnet } \\
+(\text { Amps })\end{array}$ & $\begin{array}{c}\text { Avg. } \Omega^{-} \\
\text {Momentum } \\
(\mathrm{GeV} / \mathrm{c})\end{array}$ & $P_{x}$ & $P_{y}$ & $P_{x}$ \\
\hline-450 & 280. & $-0.054 \pm 0.040$ & $-0.043 \pm 0.041$ & $-0.080 \pm 0.050$ \\
& 319. & $-0.053 \pm 0.036$ & $0.017 \pm 0.040$ & $0.032 \pm 0.046$ \\
& 368. & $-0.068 \pm 0.030$ & $0.020 \pm 0.035$ & $-0.018 \pm 0.034$ \\
\cline { 2 - 5 } & 325 (avg.) & $-0.060 \pm 0.020$ & $0.004 \pm 0.022$ & $-0.016 \pm 0.024$ \\
\hline-1000 & 400 (avg.) & $-0.053 \pm 0.054$ & $-0.073 \pm 0.056$ & $-0.077 \pm 0.059$ \\
\hline
\end{tabular}

Table 5.13: Phase 2. Polarization results at $\pm 2 \mathrm{mR}$ targeting angle for the $\Omega^{-}$data. It is assumed that $\gamma_{\Omega^{-}}=+1$. If $\gamma_{\Omega}=-1$, the polarizations and uncertainties are scaled by $-5 / 3$.

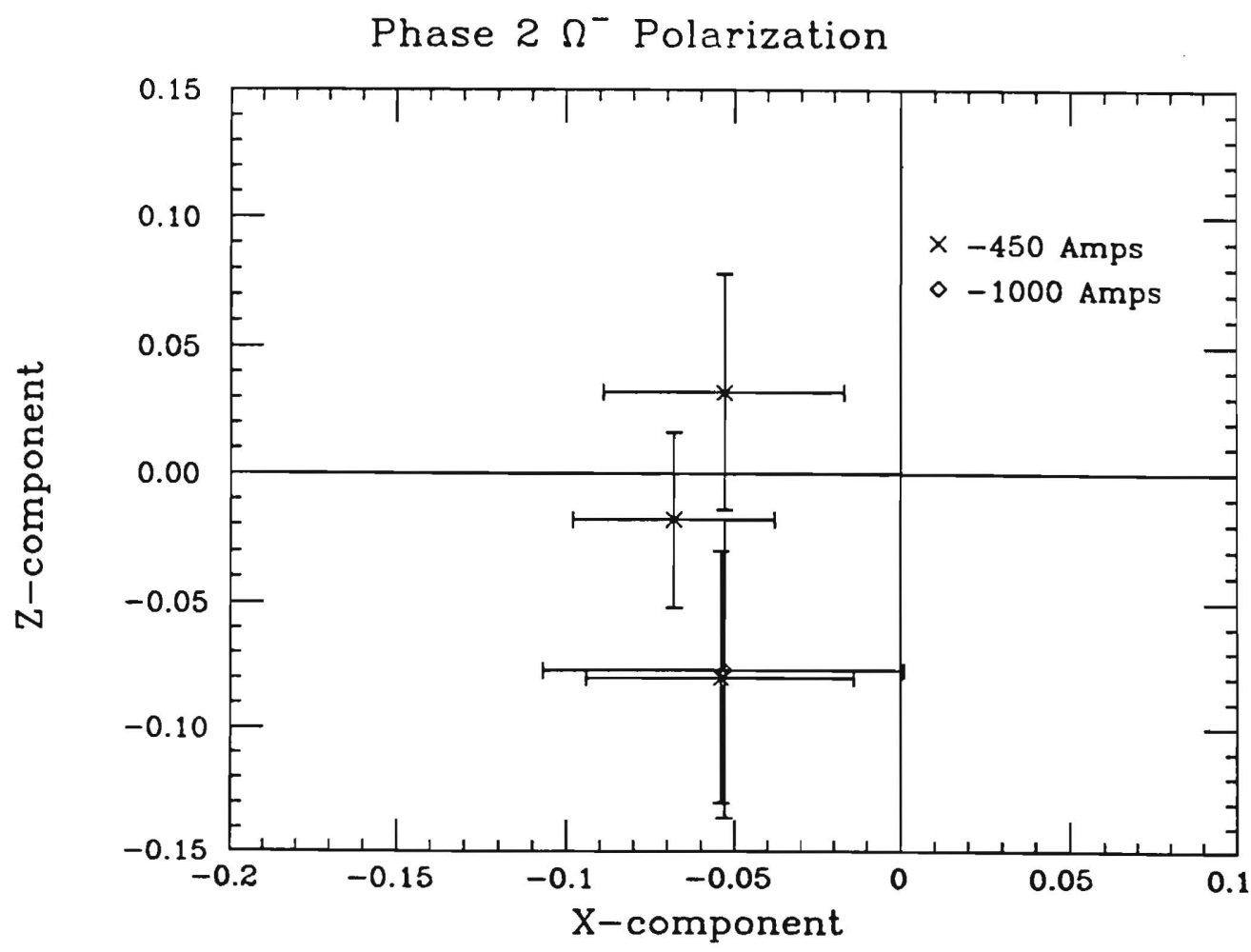

Figure 5.9: Phase $2 \Omega^{-}$Polarization components. Plotted are $P_{x}$ vs. $P_{z}$ for the 4 data points in Table 5.13. The results are presented assuming $\gamma=+1$. If $\gamma_{\Omega}$ is -1 , scale the polarization and uncertainties by $-5 / 3$. 


\begin{tabular}{|c|c|c|}
\hline $\begin{array}{c}\text { Hyperon } \\
\text { Magnet } \\
\text { (Arnps) }\end{array}$ & $\begin{array}{c}\text { Avg. } \Omega^{-} \\
\text {Momentum } \\
(\mathrm{GeV} / \mathrm{c})\end{array}$ & Polarization \\
\hline-4.50 & 280. & $-0.063 \pm 0.047$ \\
& 319. & $-0.063 \pm 0.042$ \\
& 368. & $-0.080 \pm 0.035$ \\
\cline { 2 - 3 } & 325. (avg.) & $-0.070 \pm 0.023$ \\
\hline-1000 & 400. (avg.) & $-0.062 \pm 0.065$ \\
\hline
\end{tabular}

Table 5.14: Phase 2. Polarization at the $\Omega^{-}$production target. These are calculated from the $P_{x}$ component of the polarization and the magnetic moment measured in the next chapter. $\gamma$ is assumed to be +1 . If it is -1 , all of the numbers should be multiplied by $-5 / 3$. 


\begin{tabular}{|c|c|c|c|c|}
\hline $\begin{array}{c}\text { Hyperon } \\
\text { Magnet } \\
\text { (Amps) }\end{array}$ & $\begin{array}{c}\text { Avg. } \Omega^{-} \\
\text {Momentum } \\
(\mathrm{GeV} / \mathrm{c})\end{array}$ & $B_{x}$ & $B_{y}$ & $B_{z}$ \\
\hline-450 & 280. & $-0.037 \pm 0.026$ & $0.042 \pm 0.026$ & $0.115 \pm 0.032$ \\
& 319. & $0.046 \pm 0.023$ & $0.034 \pm 0.026$ & $0.071 \pm 0.030$ \\
& 368. & $0.070 \pm 0.019$ & $-0.044 \pm 0.022$ & $0.087 \pm 0.022$ \\
\cline { 2 - 5 } & 325. (avg.) & $0.032 \pm 0.013$ & $0.006 \pm 0.014$ & $0.092 \pm 0.015$ \\
\hline-1000 & 400. (avg.) & $0.004 \pm 0.035$ & $0.066 \pm 0.087$ & $0.124 \pm 0.038$ \\
\hline
\end{tabular}

Table 5.15: Phase 2. Bias results at $\pm 2 \mathrm{mR}$ targeting angle for the $\Omega^{-}$data.

\begin{tabular}{|c|c|c|c|c|}
\hline $\begin{array}{c}\text { Hyperon } \\
\text { Magnet } \\
\text { (Amps) }\end{array}$ & $\begin{array}{c}\text { Avg. } \Omega^{-} \\
\text {Momentum } \\
(\mathrm{GeV} / \mathrm{c})\end{array}$ & $B_{x}$ & $B_{y}$ & $B_{z}$ \\
\hline \multirow[t]{4}{*}{-450} & 280. & $0.018 \pm 0.046$ & $0.033 \pm 0.049$ & $0.049 \pm 0.063$ \\
\hline & 319. & $0.029 \pm 0.043$ & $-0.024 \pm 0.049$ & $0.010 \pm 0.055$ \\
\hline & 372. & $0.030 \pm 0.032$ & $-0.062 \pm 0.036$ & $0.104 \pm 0.033$ \\
\hline & 332.(avg.) & $0.028 \pm 0.026$ & $-0.027 \pm 0.026$ & $0.074 \pm 0.026$ \\
\hline-1000 & 408.(avg.) & $0.125 \pm 0.078$ & $-0.027 \pm 0.090$ & $0.019 \pm 0.098$ \\
\hline
\end{tabular}

Table 5.16: Phase 2. Bias results at $0 \mathrm{mR}$ targeting angle for the $\Omega^{-}$data. These are to be compared with Table 5.15 . 


\begin{tabular}{|c|c|c|c|c|c|c|}
\hline $\begin{array}{l}\text { Hyperon } \\
\text { Magnet } \\
\text { (Amps) }\end{array}$ & $\begin{array}{l}\text { Tgting } \\
\text { Angle } \\
(\mathrm{mR})\end{array}$ & $\begin{array}{l}\text { Avg. } \Omega^{-} \\
\text {Momentum } \\
(\mathrm{GeV} / \mathrm{c})\end{array}$ & $\begin{array}{c}\text { Final } \\
\text { Event } \\
\text { Sample }\end{array}$ & $\chi_{x}^{2}$ & $\chi_{y}^{2}$ & $\chi_{z}^{2}$ \\
\hline \multirow[t]{12}{*}{-450} & \multirow[t]{4}{*}{+2.0} & 280. & 3133 & 35.5 & 35.0 & 41.6 \\
\hline & & 319. & 2883 & 26.9 & 63.3 & 47.3 \\
\hline & & 368. & 3460 & 31.7 & 30.0 & 47.1 \\
\hline & & 323.(avg.) & 9476 & 55.9 & 93.0 & 105. \\
\hline & \multirow[t]{4}{*}{0.0} & 281. & 1744 & 23.8 & 35.3 & 58.4 \\
\hline & & 319. & 1661 & 36.3 & 23.4 & 31.9 \\
\hline & & 372. & 2811 & 47.4 & 31.4 & 52.2 \\
\hline & & 332.(avg.) & 6216 & 55.1 & 55.1 & 86.4 \\
\hline & \multirow[t]{4}{*}{-2.0} & 280. & 3099 & 35.4 & 38.4 & 21.5 \\
\hline & & 319. & 2894 & 24.6 & 27.1 & 23.7 \\
\hline & & 368. & 3970 & 20.5 & 54.4 & 38.9 \\
\hline & & 325.(avg.) & 9963 & 26.8 & 70.8 & 56.2 \\
\hline \multirow[t]{2}{*}{-1000} & +2.0 & 400.(avg.) & 1403 & 26.1 & 44.4 & 40.6 \\
\hline & -2.0 & 400.(avg.) & 1167 & 14.6 & 26.7 & 20.5 \\
\hline
\end{tabular}

Table 5.17: Phase 2. Statistics of the $\Omega^{-}$polarization results. The results are shown as a function of momentum bin for the $-450 \mathrm{Amp}$ sample. Only averages over all momentum are presented for the -1000 Amp sample because of the low number of events. 


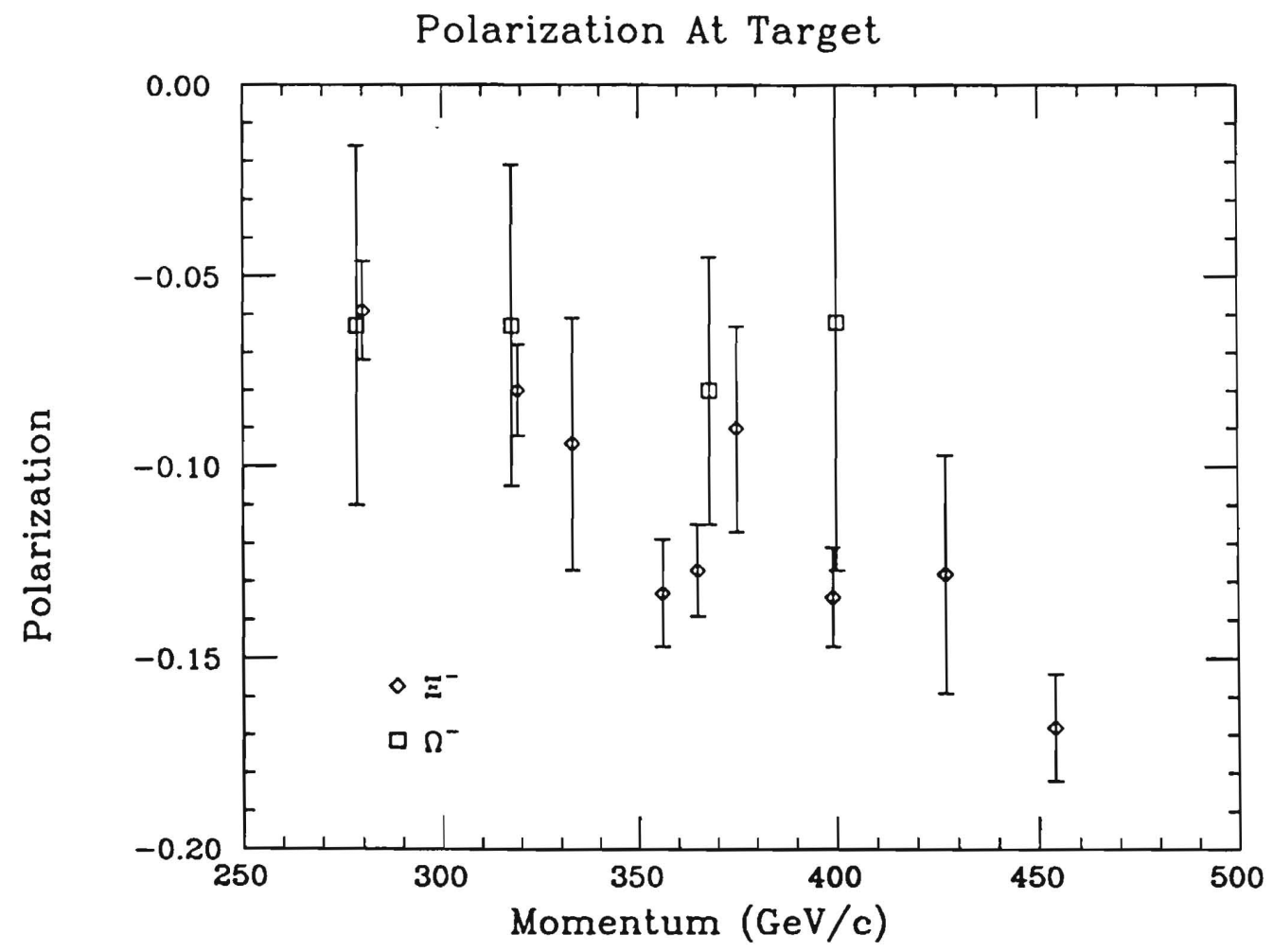

Figure 5.10: Phase 2. $\Omega^{-}$polarization at the production target as a function of momentum. Again, $\gamma_{\Omega}$ is assumed to be +1 . For comparison, the $\Xi^{-}$polarization from the next section is included. 


\subsection{Phase 2 Cascade Minus Polarization Results}

The Hybrid Monte Carlo procedure was carried out for the Phase $2 \Xi^{-}$data. The same aperture and separation cuts used in analyzing the Phase $1 \Xi^{-}$sample was used in this analysis.

The results of the polarization analysis are as follows. Table 5.18 shows the asymmetry results of the Hybrid M.onte Carlo. Table 5.19 shows the polarization components for three momentum bins at each field integral for the $\pm 2 \mathrm{mR}$ data. These were found by combining the results of Equation 5.25 with the relation between $P_{\Lambda}$ and $P_{\Xi}$ (Equation 5.20). Table 5.20 shows the polarization at the $\Xi^{-}$production target, before it precesses in the Hyperon Magnet. Table 5.21 shows the bias (Equation 5.26) removed from the $\pm 2 \mathrm{mR}$ data. Table 5.22 shows bias measured from the $0 \mathrm{mR}$ data. Any asymmetry signal measured in this data is all bias since that is produced by unpolarized hyperons. The asymmetries of Tables 5.18 and 5.22 are graphed in Figures 5.11 to 5.13. $P_{x}$ vs. $P_{z}$ is plotted in Figure 5.14. Lastly, Table 5.23 gives the statistics of the Hybrid Monte Carlo fits to the data. The results are that the $\Xi^{-}$are significantly polarized. The weighted average polarization is $-0.112 \pm 0.005 \%$. The magnitude and direction of the $\Xi^{-}$polarization is similar to that expected in the neutral beam. The kinematic dependance of the polarization is also similar to that expected in the neutral beam in that the polarization increases with momentum. Since the $\Xi^{-}$production was at $0 \mathrm{mR}$, the polarization of the neutral beam was transferred to the $\Xi^{-}$with the exchanged quarks. There is no evidence for any parity-violating $\hat{y}$ component in the polarization. 

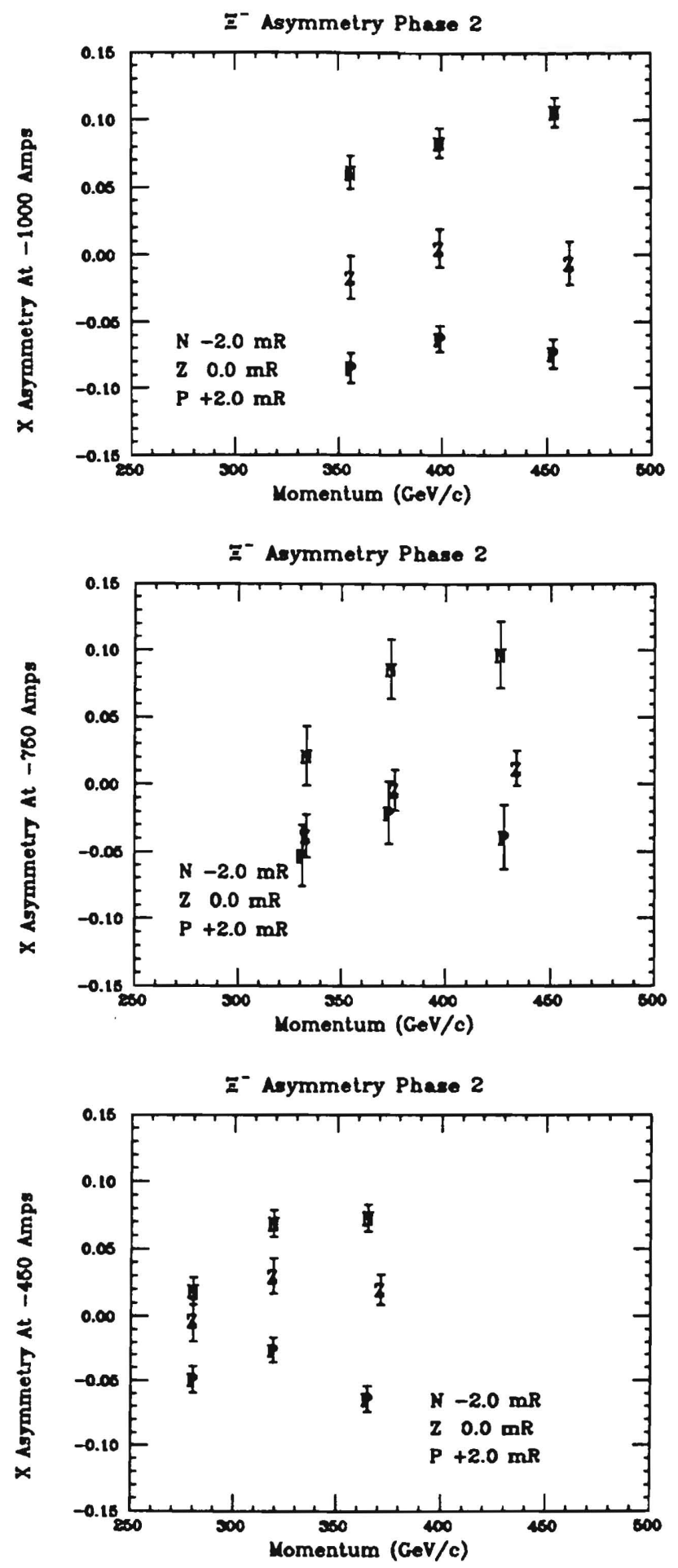

Figure 5.11: $\hat{x}$ Asymmetry of Phase $2 \Xi^{-}$s. This is the main component of $\alpha_{\Lambda} P_{\Lambda}$. The asymmetry is shown as a function of $\Xi^{-}$momentum for the 3 Hyperon Magnet currents and 3 targeting angles. 


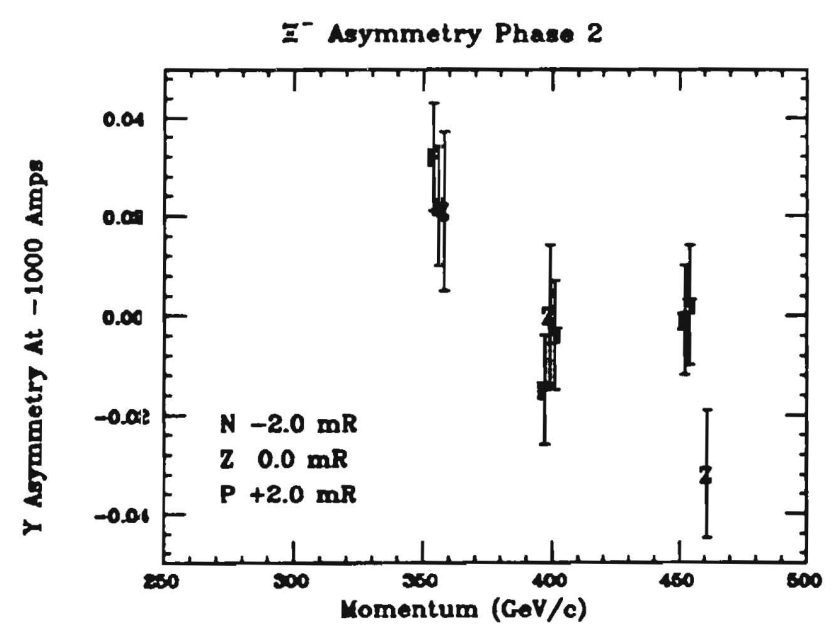

$\Xi^{-}$Asymmetry Phase 2

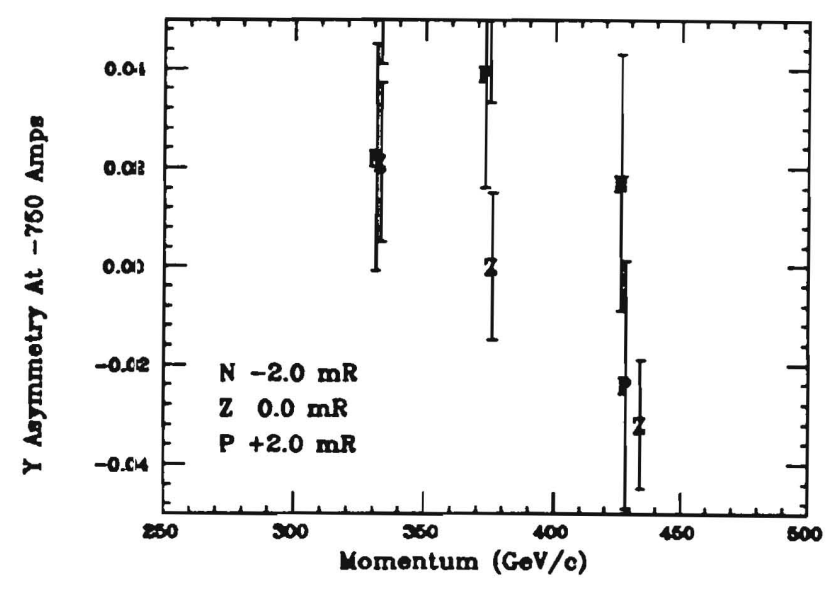

I- Asymmetry Phase 2

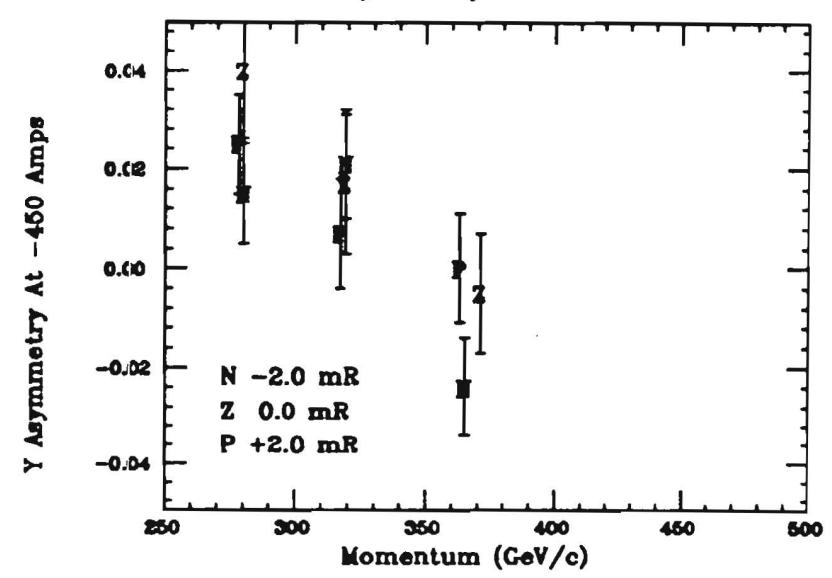

Figure 5.12: $\hat{y}$ Asymmetry of Phase $2 \Xi^{-} \mathrm{s}$. This is the parity-violating component of $\alpha_{\Lambda} P_{\Lambda}$. The asymmetry is shown as a function of $\Xi^{-}$momentum for the 3 Hyperon Magnet currents and 3 targeting angles. 
E- Asymmetry Phase 2

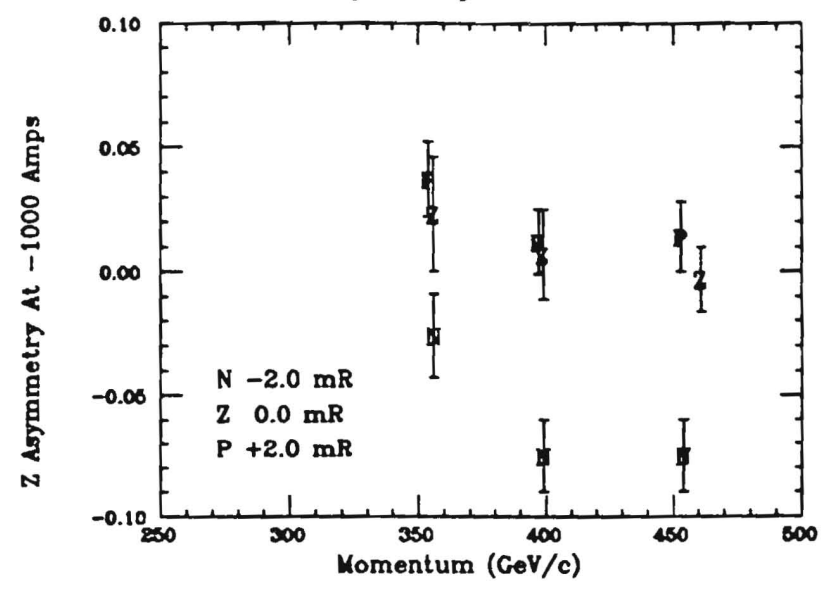

E- Asymmetry Phase 2

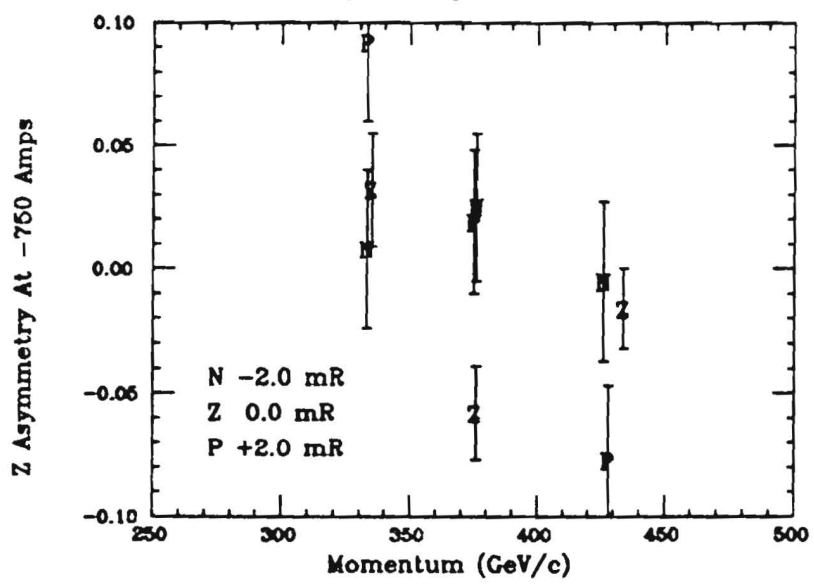

ב- Asymmetry Phase 2

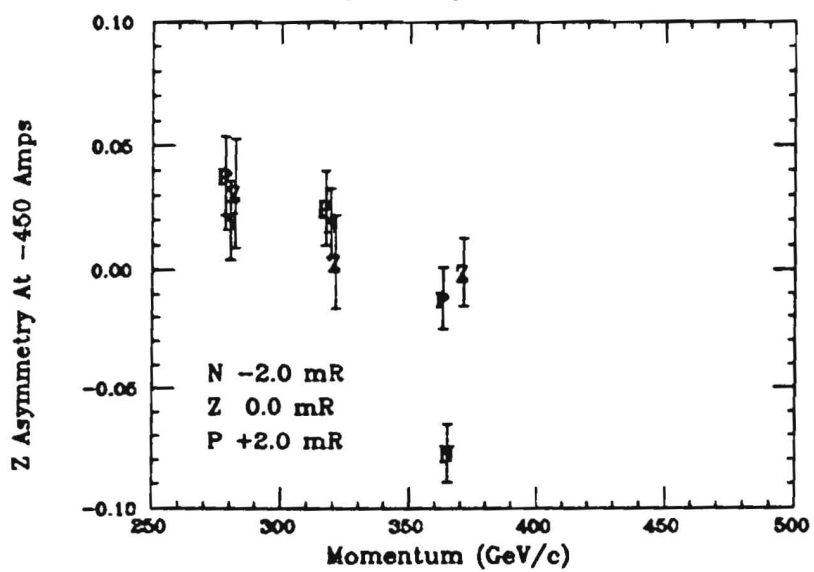

Figure 5.13: $\hat{z}$ Asymmetry of Phase $2 \Xi^{-}$s. The $\hat{x}$ component of $\alpha_{\Lambda} P_{\Lambda}$ precesses onto this axis in the Hyperon Magnet. The asymmetry is shown as a function of momentum for the the 3 Hyperon Magnet currents and 3 targeting angles. 


\begin{tabular}{|c|c|c|c|c|c|}
\hline $\begin{array}{l}\text { Hyperon } \\
\text { Magnet } \\
\text { (Amps) }\end{array}$ & $\begin{array}{l}\text { Tgting } \\
\text { Angle } \\
(\mathrm{mR})\end{array}$ & $\begin{array}{l}\text { Momentum } \\
(\mathrm{GeV} / \mathrm{c})\end{array}$ & $\begin{array}{c}\text { Asymmetry } \\
\hat{x} \\
\text { Component }\end{array}$ & $\begin{array}{c}\text { Asymmetry } \\
\hat{y} \\
\text { Component }\end{array}$ & $\begin{array}{c}\text { Asymmetry } \\
\hat{z} \\
\text { Component }\end{array}$ \\
\hline \multirow{6}{*}{-450} & \multirow{3}{*}{+2.0} & 280 & $-0.049 \pm 0.010$ & $0.025 \pm 0.010$ & $0.038 \pm 0.016$ \\
\hline & & $3 \overline{19}$ & $-0.026 \pm 0.010$ & $0.007 \pm 0.011$ & $0.025 \pm 0.015$ \\
\hline & & $3 \overline{65}$ & $-0.064 \pm 0.010$ & $-0.000 \pm 0.011$ & $-0.012 \pm 0.013$ \\
\hline & \multirow[t]{3}{*}{-2.0} & 230 & $0.019 \pm 0.010$ & $0.015 \pm 0.010$ & $0.020 \pm 0.016$ \\
\hline & & $3 \overline{19}$ & $0.069 \pm 0.010$ & $0.021 \pm 0.011$ & $0.019 \pm 0.014$ \\
\hline & & 365 & $0.073 \pm 0.010$ & $-0.024 \pm 0.010$ & $-0.077 \pm 0.012$ \\
\hline \multirow[t]{6}{*}{-750} & \multirow[t]{3}{*}{+2.0} & 3.33 & $-0.053 \pm 0.023$ & $0.022 \pm 0.023$ & $0.092 \pm 0.032$ \\
\hline & & $3 \overline{75}$ & $-0.021 \pm 0.023$ & $0.039 \pm 0.023$ & $0.020 \pm 0.029$ \\
\hline & & 428 & $-0.039 \pm 0.024$ & $-0.024 \pm 0.025$ & $-0.077 \pm 0.030$ \\
\hline & \multirow[t]{3}{*}{-2.0} & 333 & $0.021 \pm 0.022$ & $0.063 \pm 0.022$ & $0.008 \pm 0.032$ \\
\hline & & 374 & $0.086 \pm 0.022$ & $0.057 \pm 0.024$ & $0.025 \pm 0.030$ \\
\hline & & 426 & $0.097 \pm 0.025$ & $0.017 \pm 0.026$ & $-0.005 \pm 0.032$ \\
\hline \multirow[t]{6}{*}{-1000} & \multirow[t]{3}{*}{+2.0} & $3 \overline{55}$ & $-0.085 \pm 0.011$ & $0.032 \pm 0.011$ & $0.037 \pm 0.015$ \\
\hline & & 399 & $-0.063 \pm 0.010$ & $-0.015 \pm 0.011$ & $0.012 \pm 0.013$ \\
\hline & & 453 & $-0.074 \pm 0.011$ & $-0.001 \pm 0.011$ & $0.014 \pm 0.014$ \\
\hline & \multirow[t]{3}{*}{-2.0} & 356 & $0.061 \pm 0.012$ & $0.022 \pm 0.012$ & $-0.026 \pm 0.017$ \\
\hline & & 399 & $0.083 \pm 0.011$ & $-0.004 \pm 0.011$ & $-0.075 \pm 0.015$ \\
\hline & & $\overline{454}$ & $0.106 \pm 0.011$ & $0.002 \pm 0.012$ & $-0.07 \overline{5} \pm 0.015$ \\
\hline
\end{tabular}

Table 5.18: Phase 2. $\Xi^{-}$asymmetry results from the Hybrid Monte Carlo. These were used in Equations 5.25 and 5.26. The results from $0 \mathrm{mR}$ are given in Table 5.22. 


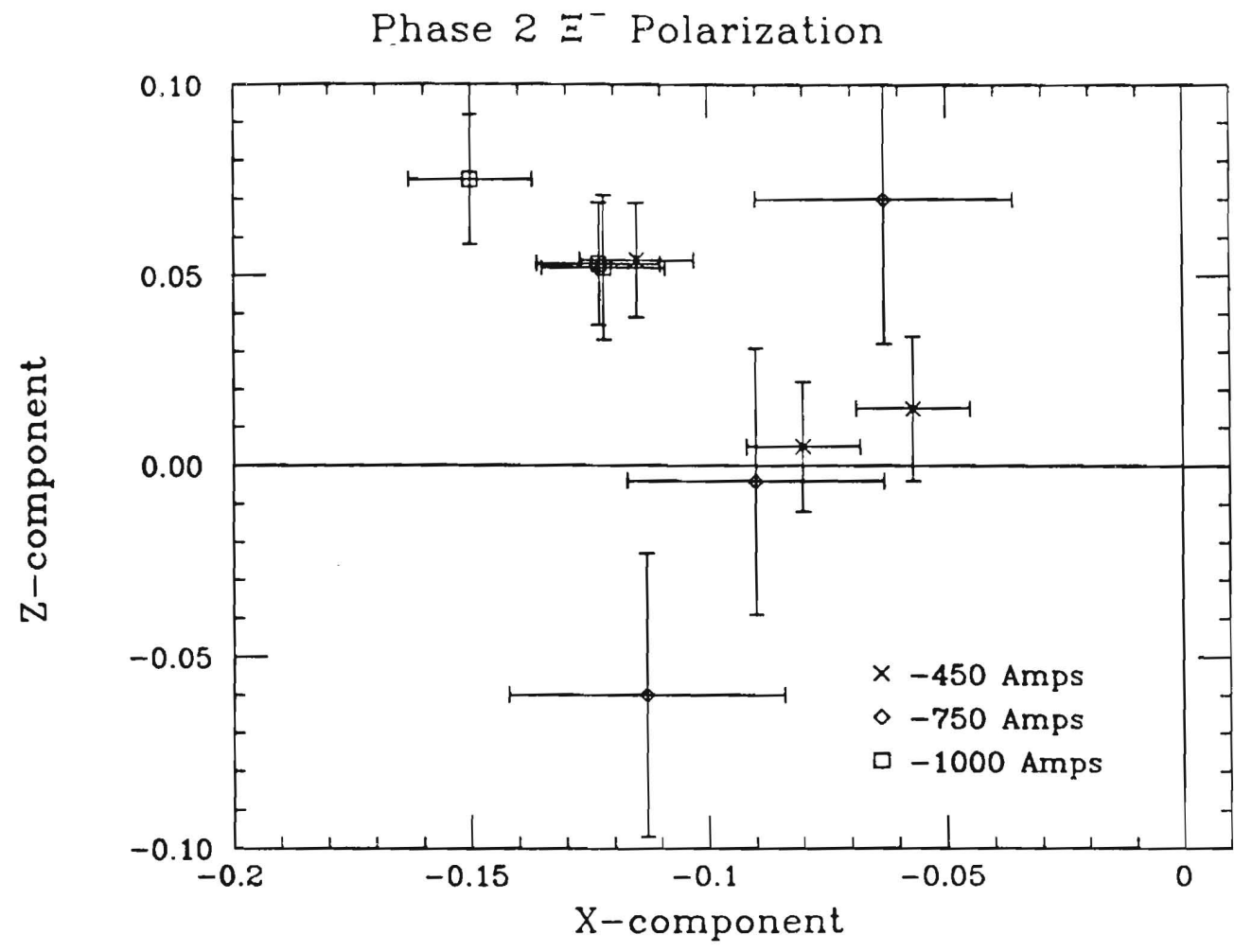

Figure 5.14: Phase $2 \Xi^{-}$Polarization components. Plotted are $P x$ vs. $P_{z}$ for the 9 data points of Table 5.19.

\begin{tabular}{|c|c|c|c|c|}
\hline $\begin{array}{c}\text { Hyperon } \\
\text { Magnet } \\
\text { (Amps) }\end{array}$ & $\begin{array}{c}\text { Avg. } \Xi^{-} \\
\text {Momentum } \\
(\mathrm{GeV} / \mathrm{c})\end{array}$ & $P_{x}$ & $P_{y}$ & $P_{z}$ \\
\hline-450 & 280. & $-0.057 \pm 0.012$ & $0.008 \pm 0.012$ & $0.015 \pm 0.019$ \\
& 319. & $-0.080 \pm 0.012$ & $-0.012 \pm 0.014$ & $0.005 \pm 0.017$ \\
& 365. & $-0.115 \pm 0.012$ & $\mathbf{0 . 0 2 0} \pm 0.012$ & $0.054 \pm 0.015$ \\
\hline-750 & 333. & $-0.063 \pm 0.027$ & $-0.035 \pm 0.027$ & $0.070 \pm 0.038$ \\
& 375. & $-0.090 \pm 0.027$ & $-0.014 \pm 0.028$ & $-0.004 \pm 0.035$ \\
& 427. & $-0.113 \pm 0.029$ & $-0.034 \pm 0.030$ & $-0.060 \pm 0.037$ \\
\hline-1000 & 356. & $-0.122 \pm 0.013$ & $\mathbf{0 . 0 0 8} \pm 0.013$ & $0.052 \pm 0.019$ \\
& 399. & $-0.123 \pm 0.013$ & $-0.009 \pm 0.013$ & $0.053 \pm 0.016$ \\
& 454. & $-0.150 \pm 0.013$ & $-0.003 \pm 0.014$ & $0.075 \pm 0.017$ \\
\hline
\end{tabular}

Table 5.19: Phase 2. $\Xi^{-}$polarization results at $\pm 2 \mathrm{mR}$ targeting angle. These are the components of the polarization after precessing in the Hyperon magnet. 


\begin{tabular}{|c|c|c|}
\hline $\begin{array}{c}\text { Hyperon } \\
\text { Magnet } \\
\text { (Amps) }\end{array}$ & $\begin{array}{c}\text { Avg. } \Xi^{-} \\
\text {Momentum } \\
(\mathrm{GeV} / \mathrm{c})\end{array}$ & Polarization \\
\hline-450 & 280. & $-0.059 \pm 0.013$ \\
& 319. & $-0.080 \pm 0.012$ \\
& 365. & $-0.127 \pm 0.012$ \\
\hline-750 & 333. & $-0.094 \pm 0.033$ \\
& 375. & $-0.090 \pm 0.027$ \\
& 427. & $-0.128 \pm 0.031$ \\
\hline-1000 & 356. & $-0.133 \pm 0.014$ \\
& 399. & $-0.134 \pm 0.013$ \\
& 454. & $-0.168 \pm 0.014$ \\
\hline
\end{tabular}

Table 5.20: Phase 2. $\Xi^{-}$polarization results at $\pm 2 \mathrm{mR}$ targeting angle. These are the initial polarizations at the $\Xi^{-}$production target. They are calculated by taking $\left(P_{x}^{2}+P_{z}^{2}\right)^{1 / 2}$. 


\begin{tabular}{|c|c|c|c|c|}
\hline $\begin{array}{c}\text { Hyperon } \\
\text { Magnet } \\
(\text { Amps })\end{array}$ & $\begin{array}{c}\text { Avg. } \Xi^{-} \\
\text {Momentum } \\
(\mathrm{GeV} / \mathrm{c})\end{array}$ & $B_{x}$ & $B_{y}$ & $B_{z}$ \\
\hline-450 & 280. & $-0.014 \pm 0.007$ & $0.020 \pm 0.007$ & $0.029 \pm 0.011$ \\
& 319. & $0.021 \pm 0.007$ & $0.014 \pm 0.008$ & $0.022 \pm 0.010$ \\
& 365. & $0.005 \pm 0.007$ & $-0.012 \pm 0.007$ & $-0.045 \pm 0.009$ \\
\hline-750 & 333. & $-0.016 \pm 0.016$ & $0.042 \pm 0.016$ & $0.050 \pm 0.023$ \\
& 375. & $0.033 \pm 0.016$ & $0.048 \pm 0.017$ & $0.023 \pm 0.021$ \\
& 427. & $0.029 \pm 0.017$ & $-0.003 \pm 0.018$ & $-0.041 \pm 0.022$ \\
\hline-1000 & 356. & $-0.012 \pm 0.008$ & $0.027 \pm 0.008$ & $0.006 \pm 0.011$ \\
& 399. & $0.010 \pm 0.008$ & $-0.010 \pm 0.008$ & $-0.020 \pm 0.010$ \\
& 454. & $0.016 \pm 0.008$ & $0.000 \pm 0.008$ & $-0.031 \pm 0.010$ \\
\hline
\end{tabular}

Table 5.21: Phase 2. $\Xi^{-}$bias results at $\pm 2 \mathrm{mR}$ targeting angle.

\begin{tabular}{|c|c|c|c|c|}
\hline $\begin{array}{c}\text { Hyperon } \\
\text { Magnet } \\
(\text { Amps })\end{array}$ & $\begin{array}{c}\text { Avg. } \Xi^{-} \\
\text {Momentum } \\
(\mathrm{GeV} / \mathrm{c})\end{array}$ & $B_{x}$ & $B_{y}$ & $B_{z}$ \\
\hline-450 & 280. & $0.003 \pm 0.014$ & $0.040 \pm 0.014$ & $0.031 \pm 0.022$ \\
& 319. & $0.030 \pm 0.013$ & $0.017 \pm 0.014$ & $0.003 \pm 0.019$ \\
& 371. & $0.020 \pm 0.011$ & $-0.005 \pm 0.012$ & $-0.001 \pm 0.014$ \\
\hline-750 & 333. & $-0.038 \pm 0.016$ & $0.021 \pm 0.016$ & $0.032 \pm 0.023$ \\
& 376. & $-0.004 \pm 0.015$ & $-0.000 \pm 0.015$ & $-0.058 \pm 0.019$ \\
& 434. & $0.012 \pm 0.013$ & $-0.032 \pm 0.013$ & $-0.016 \pm 0.016$ \\
\hline-1000 & 356. & $-0.017 \pm 0.016$ & $0.019 \pm 0.016$ & $0.023 \pm 0.023$ \\
& 399. & $0.005 \pm 0.014$ & $-0.014 \pm 0.014$ & $0.007 \pm 0.018$ \\
& 461. & $-0.006 \pm 0.016$ & $0.005 \pm 0.013$ & $-0.003 \pm 0.013$ \\
\hline
\end{tabular}

Table 5.22: Phase 2. $\Xi^{-}$polarization (bias) results at $0 \mathrm{mR}$ targeting angle. These results are to be compared with the bias measured at $\pm 2 . \mathrm{mR}$, shown in Table 5.8. 


\begin{tabular}{|c|c|c|c|c|c|c|}
\hline $\begin{array}{c}\text { Hyperon } \\
\text { Magnet } \\
\text { (Amps) }\end{array}$ & $\begin{array}{l}\text { Tgting } \\
\text { Angle } \\
(\mathrm{mR})\end{array}$ & $\begin{array}{c}\text { Avg. } \Xi^{-} \\
\text {Monentum } \\
(\mathrm{GeV} / \mathrm{c})\end{array}$ & $\begin{array}{c}\text { Final } \\
\text { Event } \\
\text { Sample }\end{array}$ & $\begin{array}{c}\chi_{x}^{2} \\
20 \text { bins }\end{array}$ & $\begin{array}{c}\chi_{y}^{2} \\
20 \text { bins }\end{array}$ & $\begin{array}{c}\chi_{z}^{2} \\
18 \text { bins }\end{array}$ \\
\hline \multirow[t]{9}{*}{-450} & \multirow[t]{3}{*}{+2.0} & 280 & 28246 & 26.9 & 28.6 & 49.1 \\
\hline & & 319. & 23914 & 13.8 & 9.55 & $\overline{36.4}$ \\
\hline & & 365. & 25774 & 32.2 & 14.7 & 46.3 \\
\hline & \multirow[t]{3}{*}{0.0} & 281. & 14403 & 25.7 & 24.4 & 27.0 \\
\hline & & 319. & 14392 & 22.1 & 16.6 & 28.8 \\
\hline & & 371. & 21100 & 30.6 & 37.0 & 38.2 \\
\hline & \multirow[t]{3}{*}{-2.0} & 280 . & 28355 & 13.4 & 22.3 & 22.4 \\
\hline & & 319. & 25126 & 31.0 & 27.1 & 46.0 \\
\hline & & 365. & 28424 & 35.5 & 24.0 & 35.4 \\
\hline \multirow[t]{9}{*}{-750} & \multirow[t]{3}{*}{+2.0} & 333. & $5 \overline{453}$ & 35.0 & 28.2 & 23.7 \\
\hline & & 376. & 5180 & 14.0 & 13.3 & 26.0 \\
\hline & & 428. & 4727 & 15.3 & 21.9 & 21.7 \\
\hline & \multirow[t]{3}{*}{0.0} & 334. & 11160 & 26.6 & 33.3 & 24.0 \\
\hline & & 376. & 12672 & 15.4 & 13.9 & 32.0 \\
\hline & & 435. & 17447 & 23.7 & 19.4 & 27.1 \\
\hline & \multirow[t]{3}{*}{-2.0} & 333. & 5419 & 20.2 & 25.2 & 23.1 \\
\hline & & 375. & 5009 & 24.9 & 28.9 & 19.1 \\
\hline & & 427. & 4119 & 24.4 & 28.9 & 19.1 \\
\hline \multirow[t]{9}{*}{-1000} & \multirow[t]{3}{*}{+2.0} & 356. & 23267 & 46.7 & 18.6 & 63.6 \\
\hline & & 399. & 25211 & 38.7 & 26.3 & 49.5 \\
\hline & & 453 & 21823 & 15.6 & 23.6 & 55.9 \\
\hline & \multirow[t]{3}{*}{0.0} & 356. & 10597 & 36.9 & 42.7 & 36.5 \\
\hline & & 400. & 13614 & 34.8 & 16.6 & 47.5 \\
\hline & & 461. & 17612 & 45.0 & 29.1 & 34.0 \\
\hline & \multirow[t]{3}{*}{-2.0} & 356. & 19579 & 24.8 & 37.2 & 37.9 \\
\hline & & 399. & 21740 & 34.7 & 13.2 & 57.7 \\
\hline & & 454. & 20009 & 30.1 & 35.0 & 40.6 \\
\hline
\end{tabular}

Table 5.23: Phase 2. Statistics of the $\Xi^{-}$polarization results. 
Analysis of the data recorded with the downstream target removed indicated that some $\Xi^{-}$and $\Omega^{-}$events originated upstream of the target location. These events could not be distinguished from those produced at the target. The probable source of these events was neutral particles which hit the walls of the downstream end of the upstream collimator (see Figure 3.3). The $\Xi^{-}$target-out sample exhibited the same polarization signal as the target-in data. That was expected because the polarization was transferred by the same mechanism as was production at the target. It is not a serious problem because the polarization precesses the full length of the Hyperon Magnet.

\subsection{Monte Carlo Studies}

The Hybrid Monte Carlo was subjected to a variety of checks. The first was to test external Monte Carlo events with a known polarization. Events were generated with no reconstruction or apparatus acceptance cuts. The Hybrid Monte Carlo, also without any acceptance cuts, correctly found the signs and magnitudes of the generated polarizations without any bias. Secondly, the $\Omega^{-}$polarization of the $-450 \mathrm{amp} \Omega^{-}$sample was put into the external Monte Carlo. This time the events were fully reconstructed and required to pass all the spectrometer and trigger cuts. The Hybrid Monte Carlo again correctly measured the polarization, and moreover, reproduced the bias of the data. Table 5.24 contains the bias measured in these Monte Carlo $\Omega^{-}$events. The Monte Carlo vs. data bias comparison is shown in Figure 5.15.

A Monte Carlo study was performed to estimate the effect on the magnetic moment

\begin{tabular}{|c|c|c|c|c|}
\hline $\begin{array}{c}\text { Hyperon } \\
\text { Magnet } \\
\text { (Amps) }\end{array}$ & $\begin{array}{c}\text { Avg. } \Omega^{-} \\
\text {Momentum } \\
(\mathrm{GeV} / \mathrm{c})\end{array}$ & $B_{\boldsymbol{x}}$ & $B_{y}$ & $B_{\boldsymbol{z}}$ \\
\hline-450 & 280. & $-.015 \pm .014$ & $.004 \pm .014$ & $.117 \pm .017$ \\
& 319. & $-.017 \pm .013$ & $.011 \pm .014$ & $.065 \pm .016$ \\
& $\mathbf{3 6 8 .}$ & $.028 \pm .011$ & $-.003 \pm .012$ & $.046 \pm .012$ \\
\hline
\end{tabular}

Table 5.24: Phase 2. Bias results from the combined $\pm 2 \mathrm{mR}$ targeting angle for the Monte Carlo $\Omega^{-}$data. These are to be compared with Table 5.13. 

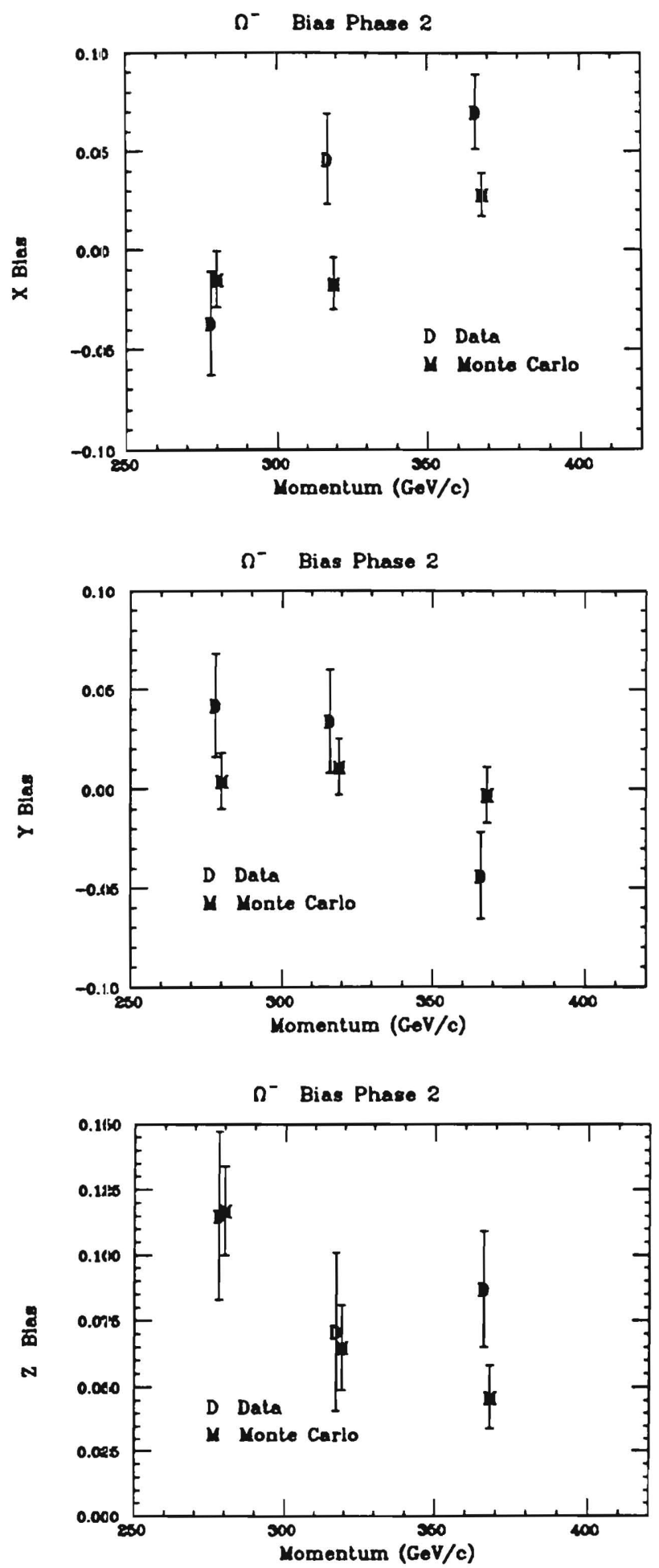

Figure 5.15: Monte Carlo Bias vs. Data Bias. The points are shown as a function of momentum for $B_{x}, B_{y}$, and $B_{z}$. 
of not using a separation cut. A Monte Carlo sample of $\Omega^{-}$events of size $8 \times$ the data was generated with the polarization measured in Phase 2. The Hybrid Monte Carlo measured the polarization as in Section 5.5 with and without the separation cut applied to the $\Xi^{-}$. The difference in the magnetic moment measured by the two methods was $0.05 \pm 0.05 \mu_{N}$.

The $\Omega^{-} \rightarrow \Xi^{-} \pi^{0}$ background estimated in Chapter 4 was included in the polarized $\Omega^{-}$Monte Carlo study. The background had no effect on the polarization or bias.

Finally, since the $\Omega^{-}$is a spin- $\frac{3}{2}$ particle, it can, in general, have rank-2 and rank-3 tensor polarizations in addition to vector (rank-1) polarization. These higher order polarizations are not measured in this thesis. In the event that it is non-zero, there is a risk that a rank-3 polarization could contribute slightly to the measured vector polarization. The distribution of protons from an ensemble of $\Omega^{-} \mathrm{s}$ was determined in Equation 5.17 which used an integral over $\Omega_{\Lambda}$, the $\Lambda$ solid angle. In reality, the acceptance is not perfect over the solid angle; in fact, the cut on $\cos \theta_{\mathrm{K}}-\phi_{\mathrm{K}}$ discussed in Section 4.3 eliminated part of the phase space entirely. A Monte Carlo study was performed in order to estimate the effect of a non-zero rank-3 tensor polarization which alternated sign with targeting angle on the measured vector polarization.

Rank 3 polarizations of \pm 0.1 were generated in the $\hat{x}$ and $\hat{z}$ directions with the Monte Carlo. The vector and rank-2 polarizations were held equal to zero. The Hybrid Monte Carlo was then used to analyze the fake events vector polarization. This determined how much the rank-3 polarization contaminated the rank- 1 measurement. The following was the result. There was no measured effect on $P_{x}$ and a slight effect on $P_{z}$. The results are quantified in Table 5.25. Assuming the $\hat{z}$ polarization measured in Phase 2 was the result of rank-1 polarization and a contaminating rank-3 polarization which was $+0.1 \hat{z}$ when the targeting angle was $-2.0 \mathrm{mR}$ and $-0.1 \hat{z}$ when the targeting angle was +2.0 $\mathrm{mR}$, the magnetic moment would be shifted by $+0.3 \pm 0.3$ nuclear magnetons. In other words, the real magnetic moment would actually be $-1.7 \mu_{N}$ instead of $-2.0 \mu_{N}$. 


\begin{tabular}{|c|c|c|}
\hline $\begin{array}{c}\text { Component } \\
\text { Direction }\end{array}$ & $\begin{array}{c}\text { M.C. Input } \\
\text { Rank-3 }\end{array}$ & $\begin{array}{c}\text { Measured } \\
\text { Vector Pol. Comp. }\end{array}$ \\
\hline$\hat{x}$ & \pm 0.1 & $-0.008 \pm 0.010$ \\
$\hat{z}$ & \pm 0.1 & $-0.014 \pm 0.011$ \\
\hline
\end{tabular}

Table 5.25: Monte Carlo Situdy. Effect of Rank-3 Tensor Polarization. The contamination of vector polarization from a rank-3 tensor polarization which alternates sign with targeting angle is shown.

The higher rank tensor polarizations are discussed further in context with spin transfer in Appendix B, Section 2. The result is that spin transfer from a polarized neutral beam is predicted, from the overlap of the neutral particle wavefunction with the $\Omega^{-}$ wavefunction, to produce no rank-2 or rank-3 tensor polarizations in any direction. Finally it is expected that there is no tensor polarization produced in either Phase 1 or Phase 2 [48]- [49]. 


\section{Chapter 6}

\section{Magnetic Moment Results}

This chapter concentrates on the $\Xi^{-}$and $\Omega^{-}$magnetic moment results. The magnetic moment is calculated from the particle's precession angle in the Hyperon Magnet field as discussed in Appendix A. In practice, the precession angle, $\phi$, is determined by

$$
\phi=\operatorname{arctangent}\left(\frac{P_{z}}{P_{x}}\right) .
$$

The uncertainty in the precession angle, $\Delta \phi$, is given by

$$
\Delta \phi=\left[\frac{\left(\Delta P_{x}\right)^{2} P_{z}^{2}+\left(\Delta P_{z}\right)^{2} P_{x}^{2}}{\left(P_{x}^{2}+P_{z}^{2}\right)^{2}}\right]^{1 / 2} .
$$

The magnetic moment, in units of $\mu_{N}$, is given by

$$
\mu_{B}=\frac{q}{e} g \frac{S}{\hbar} \frac{m_{p}}{m_{B}}
$$

where $g$ is the $g$-factor. The $g$-factor is related to the precession angle by

$$
g=2\left(1+\frac{\phi}{k \int B \overline{d l}}\right)
$$

where $\mathrm{k}$ is -0.227 radians per Tesla-meter for the $\Xi^{-}$and -0.179 radians per Tesla-meter for the $\Omega^{-}$. It is important to notice that if the precession angle is small, the the main contribution to the magnetic moment comes from the first term of this equation.

This chapter presents the magnetic moment results. The Phase $2 \Xi^{-}$results are presented first. Then the Phase $2 \Omega^{-}$results.

\subsection{Cascade Minus Magnetic Moment Result}

As precise measurements of the $\Xi^{-}$magnetic moment have been made in the past, there is no ambiguity in the initial direction of the polarization or the precession direction. 


\begin{tabular}{|c|c|c|}
\hline $\begin{array}{c}\text { Field Integral } \\
\text { (Tesla-Meters) }\end{array}$ & $\begin{array}{c}\text { Momentum Bin } \\
(\mathrm{GeV} / \mathrm{c})\end{array}$ & $\begin{array}{c}\text { Precession Angle } \\
\text { (Radians) }\end{array}$ \\
\hline \multicolumn{3}{|c|}{ Phase 1 } \\
\hline-15.30 & 330. & $-0.166 \pm 0.098$ \\
-19.43 & 393. & $-0.224 \pm 0.088$ \\
-22.18 & 433. & $-0.520 \pm 0.073$ \\
-25.6 .2 & 464. & $-0.503 \pm 0.085$ \\
& 485. & $-0.39 \pm 0.13$ \\
\hline-14.43 & Phase 2 \\
& 280. & $-0.28 \pm 0.34$ \\
& 319. & $-0.12 \pm 0.21$ \\
& 365. & $-0.39 \pm 0.12$ \\
\hline-17.87 & 333. & $-0.87 \pm 0.35$ \\
& 375. & $0.08 \pm 0.63$ \\
& 427. & $0.35 \pm 0.27$ \\
\hline-19.43 & 356. & $-0.44 \pm 0.15$ \\
& 398. & $-0.39 \pm 0.12$ \\
& 454. & $-0.35 \pm 0.11$ \\
\hline
\end{tabular}

Table 6.1: Phase 1 and Phase 2. $\Xi^{-}$precession angles.

The Hyperon Magnet field points in the $-\hat{y}$ direction. The $\Xi^{-}$precesses a fraction of one turn counter-clockwise around the field starting in the $-\hat{x}$ direction and precessing into the $+\hat{z}$ direction. Table 6.1 shows the precession angle as a function of momentum bin and field integral for Phase 1 and 2. These are also plotted in Figure 6.1. A least-squares fit of the precession angle vs. field integral provided a measurement of the $\mathrm{g}$-factor. The fit was constrained to the origin. The $\mathrm{g}$-factor is $1.831 \pm 0.014$ with a $\chi^{2}$ of 23.1 for 14 degrees of freedom. This leads to a magnetic moment of $-0.650 \pm 0.005$ nuclear magnetons. Separately, Phase 2 resulted in a magnetic moment of $-0.645 \pm 0.009$ nuclear magnetons with a $\chi^{2}$ of 15.7 for 9 degrees of freedom. Phase 1 resulted in a magnetic moment of $-0.652 \pm 0.006$ nuclear magnetons with a $\chi^{2}$ of 6.92 for 5 degrees of freedom.

The magnetic moment varied by less than .002 nuclear magnetons when the $\hat{y}$ aperatures were tightened by $1 \mathrm{~cm}$ in the Hybrid Monte Carlo.

The difference of the g-factor from exactly 2.0 is $0.169 \pm 0.014$. Thus, a systematic 


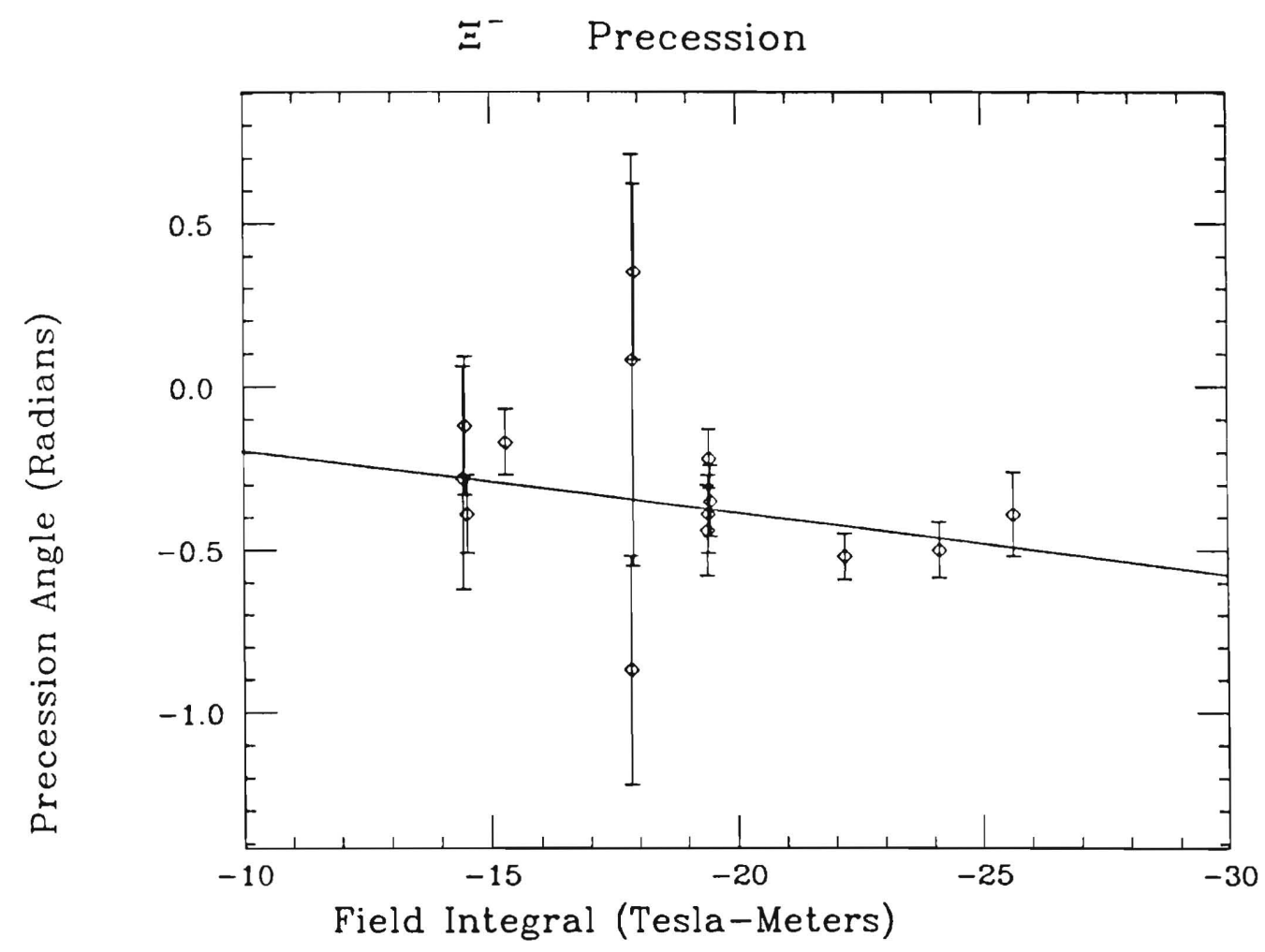

Figure 6.1: Phase $2 \Xi^{-}$precession angles vs. field integral. The precession angle is provided in radians and the field integral in tesla-meters. The fit was constrained to the origin. 


\begin{tabular}{|c|c|c|}
\hline $\begin{array}{c}\text { Field Integral } \\
\text { (Tesla-Meters) }\end{array}$ & $\begin{array}{c}\text { Momentum Bin } \\
(\mathrm{GeV} / \mathrm{c})\end{array}$ & $\begin{array}{c}\text { Precession Angle } \\
\text { (Radians) }\end{array}$ \\
\hline-14.48 & 280. & $0.98 \pm 0.45$ \\
& 319. & $-0.54 \pm 0.70$ \\
& 368. & $0.27 \pm 0.48$ \\
& 325 (avg.) & $0.27 \pm 0.38$ \\
\hline$-19.4:$ & 400 (avg.) & $0.98 \pm 0.60$ \\
\hline
\end{tabular}

Table 6.2: Phase 2. $\Omega^{-}$precession angles.

shift of the all the Hyperon Magnet field integrals of $1 \%$ would change the magnetic moment by about 0.0007 nuclear magnetons.

\subsection{Omega Minus Magnetic Moment Result}

The $\Omega^{-}$polarization comes from spin transfer from a beam with a polarization in the $-\hat{x}$ direction. The direction of the $\Omega^{-}$polarization is ambiguous because it depends on the sign of $\gamma_{\Omega}$. Fortunately, the magnetic moment and it's uncertainty do not depend on the sign of $\gamma$ because the precession angle is determined from the ratio of polarizations. Nevertheless, both possibilities are considered in this section.

If $\gamma$ is 1 , the polarization starts in the $-\hat{x}$ direction and precess into the $-\hat{z}$ direction. If $\gamma$ is -1 , the polarization starts in the $+\hat{x}$ direction and precesses into the $+\hat{z}$ direction. The same precession angle is obtained in either case. This precession is in the direction opposite to the $\Xi^{-}$. Table 6.2 contains the precession angle as a function of momentum bin and field integral for the two highest statistics samples. The average over momentum is shown for these as well as for the third field integral. Figure 6.2 contains a plot of these results. 


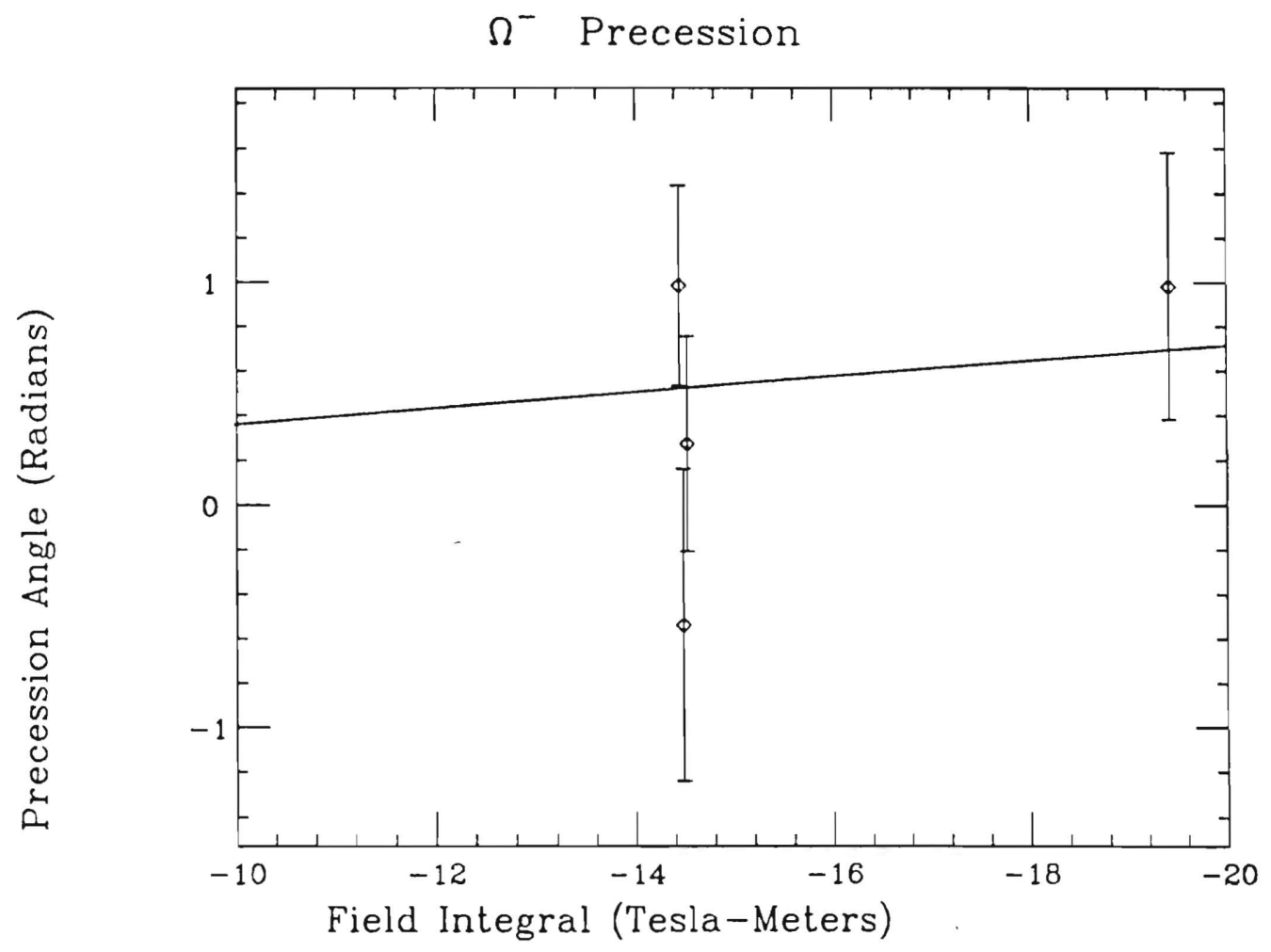

Figure 6.2: Phase $2 \Omega^{-}$precession angle vs. field integral. This fit was constrained at the origin. 
A least-squares fit of the precession angle vs. field integral provided a measurement of the g-factor. When the fit line is constrained to go through the origin, the g-factor is $2.40 \pm 0.19$ with a $\chi^{2}$ of 3.89 for the 4 data points comprised by the 3 momentum bins at -14.48 Tesla-meters and the average of the data at -19.43 Tesla-meters. Assuming the $\Omega^{-}$is spin-3/2 leads to a magnetic moment of $-2.02 \pm 0.16$ nuclear magnetons.

The precession angles of Table 6.2 assume the polarization started in the $-\hat{x}$ direction (for $\gamma=+1$ ). An ambiguity of $\pm \pi, \pm 2 \pi$, etc... exists on this initial direction. Table 6.3 lists the ambiguities, their $\chi^{2} \mathrm{~s}$, and the magnetic moment determined from each assumption. The most favored possibility is that the polarization started in the $-\hat{x}$ direction and precessed a fraction of 1 turn into the $-\hat{z}$ direction. The other possibilities are less likely and lead to magnetic moment results far from the expectations of the models discussed in Section 2.4.

A second $\chi^{2}$ was formed by fitting the polarization components of Table 5.11 to a magnetic moment and ore average polarization. Specifically,

$$
\chi^{2}=\sum_{i} \cdot\left[\frac{\left(P \cos \theta^{i}-P_{x}^{i}\right)^{2}}{\left(\delta P_{x}^{i}\right)^{2}}+\frac{\left(P \sin \theta^{i}-P_{z}^{i}\right)^{2}}{\left(\delta P_{z}^{i}\right)^{2}}\right],
$$

where $\theta$ was determined from the field integral and the magnetic moment (at the point of the fit). Figure 6.3 is a contour plot showing lines of constant $\chi^{2}$ where the horizontal axis is the polarization and the vertical axis is the magnetic moment. The lowest minimum is for a magnetic moment close to -2 . with a polarization of -0.065 . The ambiguities at $\pm \pi$ are also shown. The ambiguities at $\pm 2 \pi$ are off scale. This plot

\begin{tabular}{|c|c|c|}
\hline$\theta=\theta+$ & $\chi^{2}$ & Magnetic Moment \\
\hline$-2 \pi$ & 16.1 & $+1.73 \pm 0.16$ \\
\hline$-\pi$ & 7.77 & $-0.14 \pm 0.16$ \\
\hline 0 & $\mathbf{3 . 8 9}$ & $-2.02 \pm 0.16$ \\
\hline$+\pi$ & 4.63 & $-3.90 \pm 0.16$ \\
\hline$+2 \pi$ & 9.50 & $-5.77 \pm 0.16$ \\
\hline
\end{tabular}

Table 6.3: $\Omega^{-}$precession angle ambiguities. There are 4 degrees of freedom. These were determined by adding (or subtracting) factors of $\pi$ from the results of Table 6.2 and performing the straight-line fit through the origin to determine the magnetic moment. 


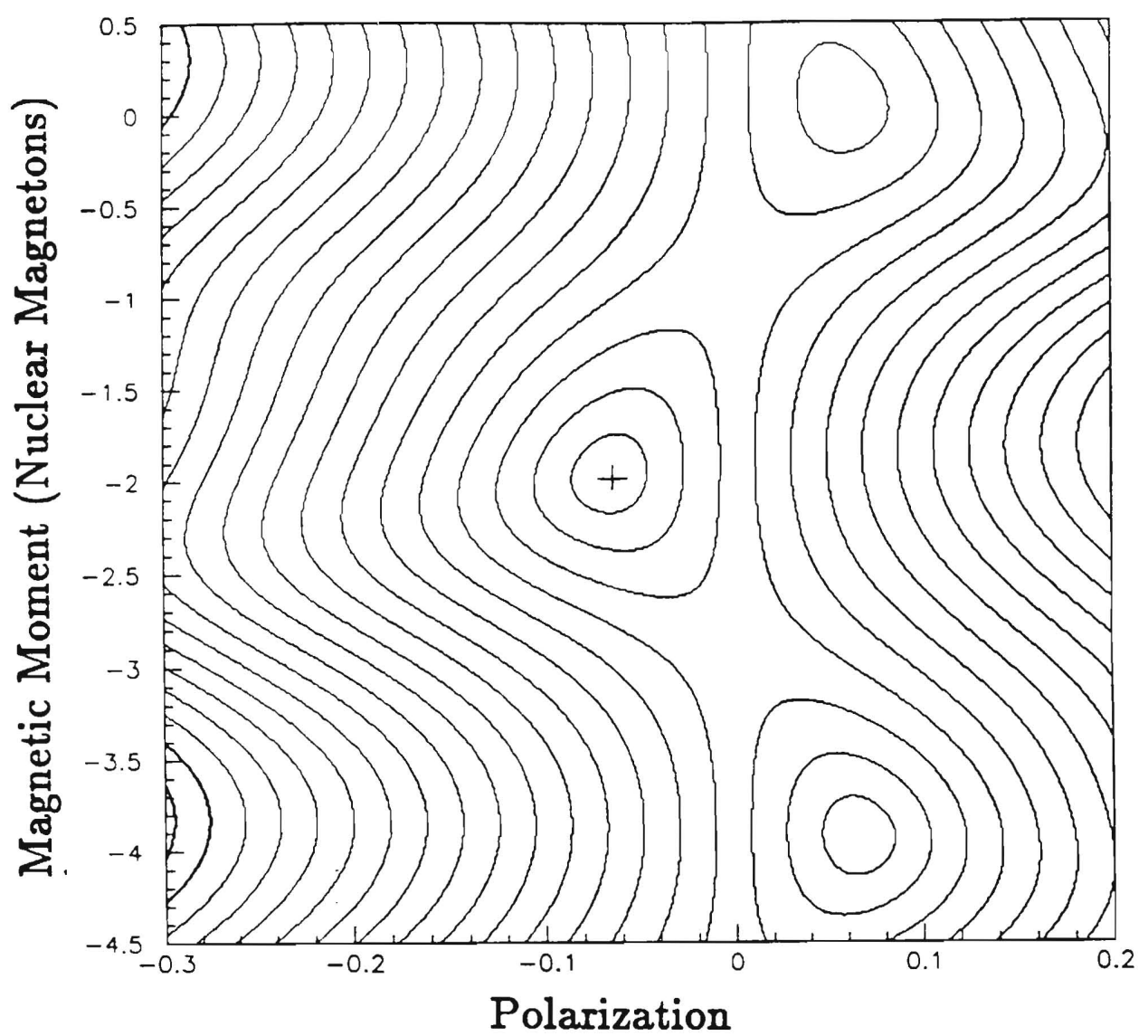

Figure 6.3: Lines of constant $\sigma$. The horizontal axis is the polarization. The vertical axis is the magnetic moment. $\gamma$ is assumed to be +1 in this plot.

shows that some values of the $\Omega^{-}$magnetic moment are excluded by more than $3 \sigma$.

The $\Omega^{-}$magnetic moment was calculated with a variation in the selection of $\Omega^{-} \mathrm{s}$. The separation cut used on the $\Xi^{-}$was applied to the $\Omega^{-}$sample and the $\Omega^{-}$decay vertex cut was taken at $0 \mathrm{~cm}$ ( rather than $50 \mathrm{~cm}$ downstream of the Hyperon Magnet ). The resulting variation in the magnetic moment was 0.12 nuclear magnetons. This is taken as the systematic error in the $\Omega^{-}$magnetic moment. Additional possible sources were discussed in Section 5.7. 


\section{Chapter 7 \\ Discussion and Conclusions}

Protons with $800 \mathrm{GeV} / \mathrm{c}$ momenta incident on a Be target at a targeting angle of \pm 2.5 $\mathrm{mR}$ produced $\Omega^{-}$with an average polarization of $-0.018 \pm 0.009$. The polarization of $\Xi^{-} \mathrm{s}$ produced at the same conditions was $-0.109 \pm .004$.

A neutral beam containing polarized hyperons was used to make polarized $\Omega^{-} \mathrm{s}$ and $\Xi^{-} \mathrm{s}$ at $0 \mathrm{mR}$. The $\Xi^{-}$polarization is in the same direction as the neutral beam. The polarization increases with $\Xi^{-}$momentum with the trend expected of the neutral beam. As the $\Xi^{-}$were produced at $0 \mathrm{mR}$, the polarization must have come from spin transfer from the neutral beam. $\Xi^{-}$polarization up to $-0.168 \pm 0.014$ was observed. The average $\Xi^{-}$polarization was $-0.112 \pm 0.005$. Polarized $\Omega^{-}$s were produced by this spin transfer method. The sign and magnitude of this polarization has an ambiguity because the sign of $\gamma_{\Omega^{-}}$has not been measured. If $\gamma_{\Omega}$ is +1 , the polarization is $-0.069 \pm .022$. If it is -1 , the polarization and the uncertainty are increased by $-5 / 3$.

The $\Xi^{-}$polarization behaves as predicted from the overlap of the naive quark model wavefunctions of the $\Xi^{-}$and a polarized neutral beam. The $\Omega^{-}$s are polarized by the same mechanism. Therefore, $\gamma_{\Omega^{-}}=+1$ is experimentally favored because the sign of the $\Omega^{-}$polarization is the same as that of the neutral beam which produced them.

The spin precession method has been used to make the first measurement of the $\Omega^{-}$ magnetic moment. The $g$-factor is $2.40 \pm 0.19 \pm 0.14$. If the $\Omega^{-}$is a spin-3/2 particle, this corresponds to a magnetic moment of $-2.02 \pm 0.16 \pm 0.12 \mu_{N}$. The first uncertainty is statistical and the second systematic. The magnetic moment and the error bar do not depend on the sign of $\gamma$.

A glance at Table 2.1 shows that this is not a high precision magnetic moment 
measurement. The uncertainty is much larger than the other measurements. However, the uncertainty excludes some of the predictions for $\mu_{\Omega}$ - reported in Table 2.4. If the $\Omega^{-}$magnetic moment is, in fact, very close to $-1.84 \mu_{N}$ (3 times the $\Lambda$ magnetic moment), a measurement with uncertainty less than 0.012 nuclear magnetons ( 3 times the uncertainty in the $\Lambda$ measurement) will be necessary to constrain the strange quark magnetic moment. If a high precision measurement of the $\Omega^{-}$magnetic moment is significantly different from $-1.84 \mu_{N}$, then corrections to the simple quark model become important. As the $\Omega^{-}$consists of three relatively heavy quarks, it provides the best hope among the long-lived baryons of a precision Lattice Gauge Theory calculation.

A high precision measurement of the $\Xi^{-}$magnetic moment has been performed. The magnetic moment was determined to be $-0.650 \pm 0.005 \pm 0.002 \mu_{N}$. This result confirms and improves upon the previous two $\Xi^{-}$magnetic moment measurements. The new world average is $-0.6508 \pm 0.0054 \mu_{N}$. This result is significantly different from the prediction of the simple quark model suggested in Table 2.4. Thus, the corrections discussed in Chapter 2 are important in the description of baryon magnetic moments.

This is the first experiment which has taken advantage of the spin-transfer method of producing polarized hyperons. Fermilab Experiment E800, which will collect data during the 1990 fixed-target run, expects to use this method to make a determination of the $\Omega^{-}$magnetic moment to 0.05 nuclear magnetons. 


\section{Appendix A}

\section{Spin Precession in a Magnetic Field}

This Appendix discusses the motion of a charged particle with spin, $S$, in a constant magnetic field perpendicular to the particle's momentum. In a coordinate system fixed with respect to the particles initial momentum, the spin vector precesses through an angle

$$
\phi=\frac{q}{\beta m c^{2}}(g / 2-1+1 / \gamma) \int B d l .
$$

The particle's momentum also changes direction. The momentum vector precesses through an angle given by

$$
\theta=\frac{q}{\beta m c^{2}} \frac{1}{\gamma} \int B d l .
$$

Thus, the spin precession in a coordinate system which precesses with the particle's momentum is

$$
\phi-\theta=\frac{q}{\beta m c^{2}}(g / 2-1) \int B d l .
$$

As $\beta \approx 1$, the net precession angles of the $\Xi^{-}$and $\Omega^{-}$are

$$
(\phi-\theta)_{\Xi-}=-0.227\left(\frac{g_{\Xi-}}{2}-1\right) \int B d l,
$$

and

$$
(\phi-\theta)_{\Omega^{-}}=-0.179\left(\frac{g_{\Omega^{-}}}{2}-1\right) \int B d l
$$

where the constant-factor is given in units Tesla - meters per radian.

Since the Hyperon Magnet field is parallel to $\hat{y}$, the assumption that the field is perpendicular to the beam direction is a good approximation. 


\section{Appendix B}

\section{Spin Transfer}

This appendix contains a model of the spin-transfer production and polarization of $\Omega^{-}$ and $\Xi^{-}$hyperons from a polarized neutral beam. The purpose for constructing this model is to make some prediction for the momentum dependence of the $\Omega^{-}$and $\Xi^{-}$ polarization in Phase 2.

The ingredients necessary for this study are: neutral particle cross-sections from production by protons, charged hyperon production cross-sections from production by various neutral particle species, a parameterization of the neutral particle polarization, the lifetime of the neutral particles, and a model for spin transfer from the neutral particles to the charged hyperons. This data is then hooked together in an equation for the polarization of the $\Xi^{-}$and $\Omega^{-}$as a function of their momentum.

\section{B.1 Neutral Particle Cross-sections}

The neutral particle cross-sections as a function of $x_{f}$ and $P_{T}$ from production by 800 $\mathrm{GeV} / \mathrm{c}$ protons are scaled up from the results of previous experiments at lower energy.

A neutral beam produced by $800 \mathrm{GeV} / \mathrm{c}$ protons contains photons, neutrons, Lambdas, neutral kaons, $\Xi^{0} \mathrm{~s}$, and neutral anti-particles. These are ordered roughly by crosssection. Some of these may be neglected in a study of $\Omega^{-}$and $\Xi^{-}$production with simple arguments. The photons are neglected because their cross-section for hyperon production is small [50]. The neutral kaons are neglected because they have strangeness -1. The anti-particles and other neutral particles are short-lived or neglected because their production cross-sections are small and (or) they have negative strangeness. The procedure in determining the neutral beam content of E756 was to scale the invariant 
Neutron Production

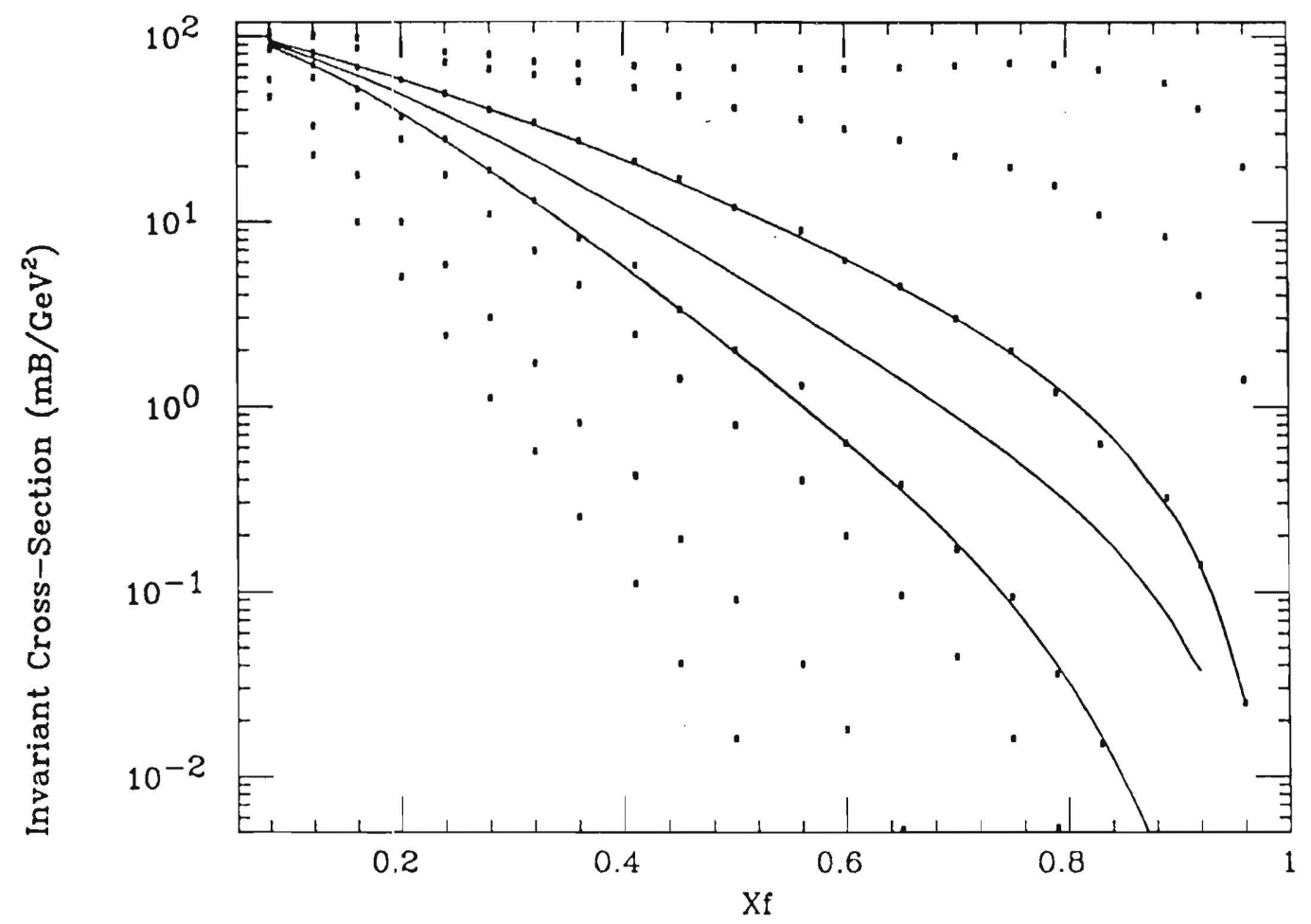

Figure B.1: Neutron production by $400 \mathrm{GeV} / \mathrm{c}$ protons. The targeting angles are 0.7 , 1.6, 3.0, 5.0, 6.0, 8.0 and $1.0 .0 \mathrm{mR}$. The points are the data. The lines are the fit at 3.0 and $5.0 \mathrm{mR}$ and the interpolation at $4.0 \mathrm{mR}$.

cross-section to $800 \mathrm{GeV} / \mathrm{c}$ and a $2 \mathrm{mR}$ production angle from data at $400 \mathrm{GeV} / \mathrm{c}$ and a $4 \mathrm{mR}$ production angle. A parameterization in $x_{f}$ and $P_{T}$ was used which fits the data closely.

Data is available for neutron[51], Lambda [52], and Cascade-zero[52] production by high-energy protons from a variety of kinematic conditions and targets. All of this data is subject to systematic uncertainties on the order of $20 \%$ originating from sources such as target absorption and mismeasuring the number of protons incident on the target.

Neutron production data, taken from Reference [51], for $400 \mathrm{GeV} / \mathrm{c}$ protons incident at various targeting angles on a $\mathrm{Cu}$ target is shown in Figure B.1. The data was estimated from the plot. The statistical uncertainties for each point were not available. The data was fit to the form

$$
E \frac{d^{3} \sigma}{d p^{3}}=\exp \left(C_{1}+C_{2} x_{f}^{2}+C_{3} x_{f}+C_{4} x_{f} P_{T}+C_{5} P_{T}^{2}+C_{6} P_{T}^{4}+C_{7} P_{T}^{6}\right)\left(1-x_{f}\right)^{C_{6}+C_{9} P_{T}^{2}}
$$




\begin{tabular}{|l|c|c|c|}
\hline & Neutron & $\Lambda$ & $\Xi^{0}$ \\
\hline$C_{1}$ & 4.83 & 3.44 & .7 \\
$C_{2}$ & 4.98 & 1.96 & 3.16 \\
$C_{3}$ & .683 & -1.79 & -2.09 \\
$C_{4}$ & .0662 & -.92 & -1.31 \\
$C_{5}$ & -4.436 & -2.21 & -1.48 \\
$C_{6}$ & 1.10 & .31 & .068 \\
$C_{7}$ & -.280 & -.023 & 0.0 \\
$C_{8}$ & 3.80 & .99 & 3.52 \\
$C_{9}$ & -.711 & .12 & -.30 \\
\hline
\end{tabular}

Table B.1: These are the constants from the parameterization of the data to Equation B.1.

Rather arbitrary uncertainties were assigned to each point in order to force the fit to behave at high $x_{f}$. The fit was poor when all of the data was included. In particular, the matchup was bad in the region of interest, at the intermediate targeting angles and high $x_{f}$. This was understandable owing to the shape of the cross-section at low $P_{T}$ and high $x_{f}$. Diffractive production processes like $p+A \rightarrow n+\pi^{+}+A$ contribute strongly in this region and cause the cross-section to remain high. In order to interpolate the data to the kinematic region important for this study, only the data provided at 3.0 and $5.0 \mathrm{mR}$ were used in the fit. Figure B.1 shows the fits to these curves and the interpolation to $4 \mathrm{mR}$ ( $400 \mathrm{GeV} / \mathrm{c}$ protons). The parameters of the fit are included in Table B.1.

Figures B.2 and B.3 show the cross-section data for $\Lambda$ and $\Xi^{0}$ production from 400 $\mathrm{GeV} / \mathrm{c}$ protons at a variety of kinematic conditions using a $\mathrm{Cu}$ target. The fits to the $\Lambda$ and $\Xi^{0}$ cross-sections provided in Reference [52] were used for this data. The parameters of the fits are provided in Table B.1. It is relevant to note that the $\Lambda$ and $\Xi^{0}$ were measured in the same experiment; therefore some systematic uncertainties cancel in the ratio of the cross sections. 


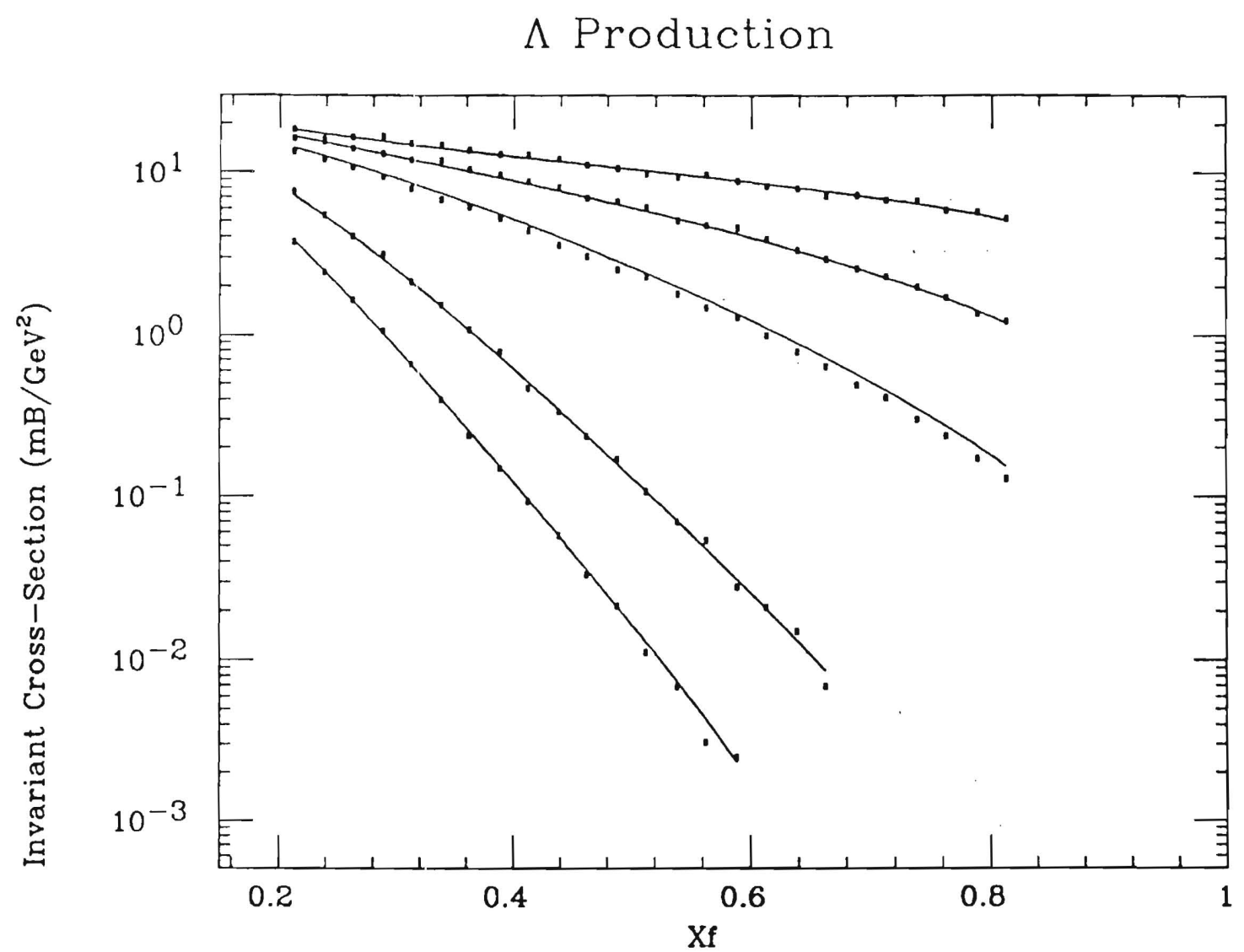

Figure B.2: $\Lambda$ production by $400 \mathrm{GeV} / \mathrm{c}$ protons. The targeting angles are $0.0,2.0,3.5$, 7.3, and $9.8 \mathrm{mR}$. The target was $\mathrm{Cu}$. The lines are the result of the fit. 


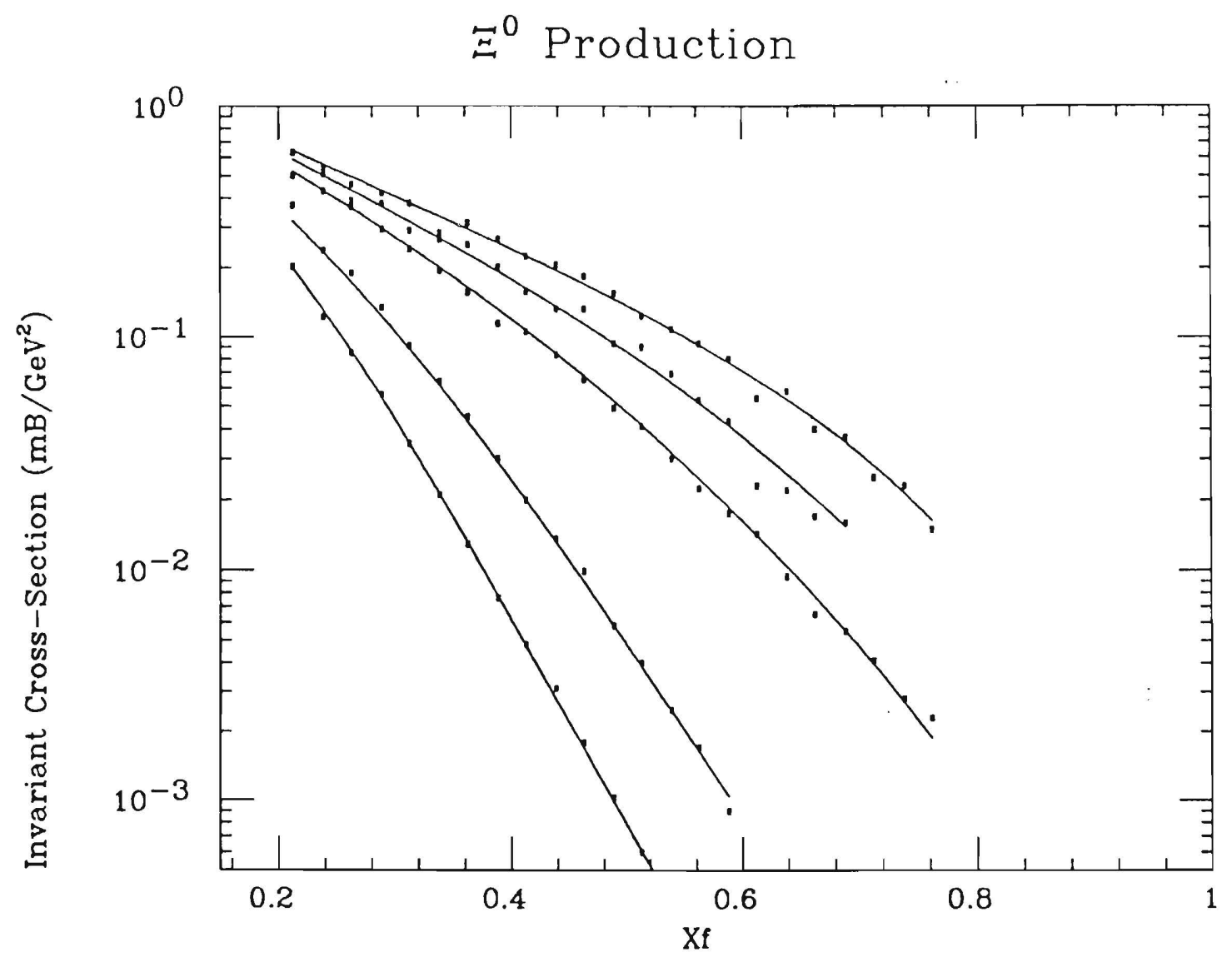

Figure B.3: $\Xi^{0}$ production by $400 \mathrm{GeV} / \mathrm{c}$ protons. The targeting angles are $0.0,2.0$, 3.5, 7.3, and $9.8 \mathrm{mR}$. The target was $\mathrm{Cu}$. The lines are the result of the fit. 
The number of particles, $N(p)$, in a momentum bin of width $\Delta p$, produced by $N_{i n c}$ incoming particles is related to the invariant cross-section by

$$
N(p)=\frac{p^{2}}{E} \frac{N_{A} L \rho}{A C} \epsilon N_{i n c}\left(E \frac{d^{3} \sigma}{d p^{3}}\right)(\Delta \Omega)(\Delta p),
$$

where,

1. $p$ and $E$ are the particle's momentum and energy,

2. $N_{A}, \rho, L$ and $A$ are Avogadro's Number, the target density, length and atomic number,

3. $C$ is a target reabsorption factor,

4. $\Delta \Omega$ is the solid angle subtended by the outgoing beam (this represents the solid angle of the collimator's defining aperture),

5. $\epsilon$ is the rest of the acceptance including the momentum-dependent acceptance of a curved channel, the trigger and the reconstruction algorithm,

6. $N_{\text {inc }}$ is the number of particles in the incoming beam, and

7. $E d^{3} \sigma / d p^{3}$ is the invariant cross-section for producing the species from the incoming beam.

\section{B.2 Neutral Beam Polarization}

The polarization of the $\Lambda$ and $\Xi^{0}$ hyperons is known. Figure 2.1, taken from Reference [5], shows hyperon polarization as a function of $x_{f}$ for a fixed targeting angle. This includes $\Lambda \mathrm{s}$ and $\Xi^{0} \mathrm{~s}$. The parameterization of the polarizations of Figure 2.1 used in this study was $\mathcal{P}=-0.40 x_{f}$. But as Be was used as the target in those experiments and $\mathrm{Cu}$ was used in Phase 2, a slight modification of this parameterization was necessary. A factor of 0.75 was used because of the $A$ dependence of the polarization as measured by Lundberg et al. [53].

The polarization of the neutrons has not been measured. 


\section{B.3 Omega Minus and Cascade Minus Production from the Neutral} Beam

No data is available for $\Omega^{-}$or $\Xi^{-}$production from $\Lambda s$, neutrons, or $\Xi^{0} s$. This information has to be guessed. A reasonable guess is that the cross-sections are similar to other processes which exchange the same quarks. For example, $\Omega^{-}$production by $\Xi^{0} s$, which involves swapping an up quark in the $\Xi^{0}$ for a strange quark from the sea, has the same quark exchange as $\Lambda$ production from protons. Similarly, $\Omega^{-}$production by $\Lambda \mathrm{s}$ is like $\Xi^{0}$ production by protons. $\Omega^{-}$production by neutrons is like $\Omega^{-}$production by protons. $\Xi^{-}$production by $\Lambda \mathrm{s}$ is like $\Lambda$ production by protons. $\Xi^{-}$production by neutrons is $\Xi^{0}$ production by protons. Finally, $\Xi^{-}$production by $\Xi^{0}$ is most like neutron production by protons.

Many of these cross-sections already have been parameterized in this appendix. $\Xi^{-}$ production by $\Xi^{0}$ needs special treatment because Phase 2 production at $0 \mathrm{mR}$ occurs in a region the parameterization for neutron production is not valid. A glance at Figure B.1 shows the cross-section is nearly flat for small targeting angles. A constant invariant cross-section of $110 \mathrm{mB} / \mathrm{GeV}^{2}$ was used.

$\Omega^{-}$production from protons has not been measured at $0 \mathrm{mR}$. The only data available is from this experiment. This analysis is in progress, but no preliminary results exist. However, a preliminary estimate of the $\Omega^{-} / \Xi^{-}$production ratio as a function of $x_{f}$ is available for a limited region in $p_{t}$. The ratio is 0.03 independent of $x_{f}$ for production at $2.5 \mathrm{mR}$ [54]. Combining this with the estimate for the $\Xi^{0}$ cross section from protons at $0 \mathrm{mR}$ provides an estimate of the $\Omega^{-}$cross section.

The model for predicting the polarization requires the relative number of neutral beam species as a function of momentum and the relative number of $\Xi^{-}$or $\Omega^{-}$which they produce. Therefore, all of the parameters related to the targets and acceptance can be ignored (set equal to 1 ). The cost is that absolute rates will no longer be available. The experiment dependent parameters remaining are the energy and targeting angle of the incoming protons, $0 \mathrm{mR}$ production of the charged beam, and the distance between 
the $\mathrm{Cu}$ targets ( 8 meters in E756). This reduces Equation B.2 to

$$
N(p) \propto p\left(E \frac{d^{3} \sigma}{d p^{3}}\right)(\Delta p)
$$

Then $N_{B}(P)$, the number of species $\mathrm{B}$ as a function of momentum, in a momentum bin of width $\Delta p$, produced by a decaying beam of species $n$, with more than one momentum, $p_{n}$, is

$$
N_{B}(p)=\int_{p_{n}=p_{B}}^{800}\left(E \frac{d^{3} \sigma}{d p^{3}}\right)_{n} p_{n}\left(E \frac{d^{3} \sigma}{d p^{3}}\right)_{B} p_{B}\left(\Delta p_{B}\right) L_{n} \delta\left(x_{n} x_{B}-\frac{p_{B}}{p_{n}}\right) d p_{n}
$$

where,

1. $x_{n}=p_{n} / p_{\text {proton }}$,

2. $x_{B}=p_{B} / p_{n}$, and

3. $L_{n}=e^{D / \gamma c r}$, where $L$ ) is the distance that beam $n$ has traveled, and $c \tau$ is the speed of light times the neutral particle's lifetime. Notice that $\gamma$ is a function of $p_{n}$.

It is clear how this relates to Phase 2 of E756. $\Omega^{-} \mathrm{s}$ and $\Xi^{-} \mathrm{s}$ were produced by a decaying mixture of neutral beam species which covered a wide momentum range. $D$ is the distance between the targets ( $\sim 8$ meters).

This results in the following predictions for relative production cross sections of $\Xi^{-}$ and $\Omega^{-}$from the neutral particles. $\Xi^{-}$are produced mostly by $\Lambda \mathrm{s}$ followed by $\Xi^{0} \mathrm{~s} . \Omega^{-} \mathrm{s}$ are produced mainly by $\Xi^{0} \mathrm{~s}$ followed by $\Lambda \mathrm{s}$. Neutrons play only a small role in both $\Xi^{-}$and $\Omega^{-}$production. One encouraging note is that the $\Xi^{-} / \Omega^{-}$production ratio is close to the value seen in Phase $2(\sim 65$ at $320 \mathrm{GeV} / \mathrm{c})$.

\section{B.4 Spin Transfer to the Omega Minus and Cascade Minus}

This section contains a review of the spin transfer predictions of Reference [42] for $\Omega^{-}$and $\Xi^{-}$polarization. The cartesian spin-projections are used to parameterize the $\Omega^{-}$spin state. Comparing the $\Lambda$ distribution from $\Omega^{-}$decays with that derived in 
PHASE 2

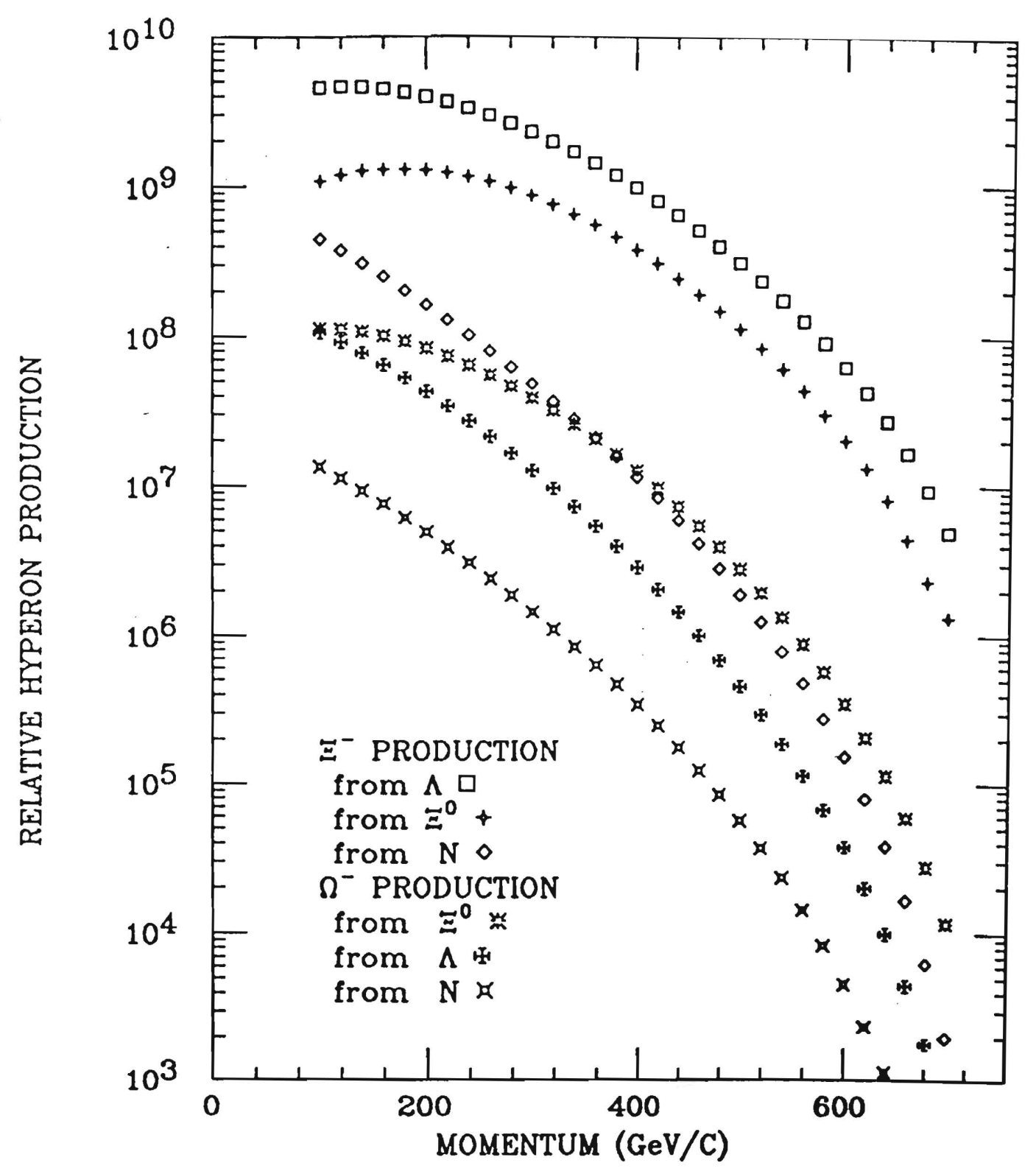
Figure B.4: Relative $\Omega^{-}$and $\Xi^{-}$rates from production by a decaying neutral beam of
widespread momentum. 
Chapter 5 leads to relations between the cartesian spin-projections and the sphericaltensor polarizations. A surnmary of the spin transfer from various polarized particles to the $\Omega^{-}$and $\Xi^{-}$is presented. The spin transfer predictions are then extended to the rank-2 and rank-3 $\Omega^{-}$polarizations.

The $\Omega^{-}$is assumed to be a spin-3/2 particle. Then, in it's helicity frame there are 4 spin projections: $\mathrm{m}=-3 / 2,-1 / 2,+1 / 2$, and $+3 / 2$. The final state wavefunction, $\psi$, for the decay to a spin-1/2 baryon and a spin- 0 meson is given by

$$
T\left|\frac{3}{2} m\right\rangle=\sum_{L=1}^{2} \sum_{\lambda=-1 / 2}^{1 / 2} A_{L}\left\langle\frac{1}{2} L \lambda \frac{1}{2}-\lambda \mid \frac{3}{2} m\right\rangle Y_{L}^{m-\lambda}(\theta, \phi)\left|\frac{1}{2} \lambda\right\rangle,
$$

where $\lambda$ is the spin projection of the daughter baryon, $\mathrm{L}$ indicates the orbital angular momentum of the daughter: particles, $A_{L}$ are the decay amplitudes, and $\theta$ and $\phi$ are the angles between the $\Omega^{-}$and the daughter baryon. If the fraction of $\Omega^{-} \mathrm{s}$ in each of the spin projections $+3 / 2,+1 / 2,-1 / 2$, and $-3 / 2$ is $A, B, C$, and $D$ respectively, then the angular distribution, $\psi^{*} \psi$, of daughter baryons is given by

$$
\begin{aligned}
& I_{\Lambda}(\theta, \phi)=\frac{3}{8 \pi} \sin ^{2} \theta(A+D)+\alpha_{\Omega}-\frac{3}{8 \pi} \sin ^{2} \theta(A-D) \cos \theta+ \\
& \frac{1}{8 \pi}\left(1+3 \cos ^{2} \theta\right)(B+C)+\alpha_{\Omega}-\frac{1}{8 \pi}(B-C)\left(9 \cos ^{3} \theta-5 \cos \theta\right) .
\end{aligned}
$$

The decay is isotropic in $\phi$.

The $\Omega^{-}$decay distribution, Equation B.6 can be compared with the distribution, Equation 5.3, obtained when the spin-state is parameterized with spherical tensor operators. This provides relations between the cartesian parameters and the spherical tensor parameters in the helicity frame. They are

$$
\begin{array}{ll}
t_{0}^{0 *}= & 1 \\
t_{1}^{0 *}= & \sqrt{\frac{1}{15}}(3 A+B-C-3 D) \\
t_{2}^{0 *}= & \sqrt{\frac{1}{5}}(A-B-C+D) \\
t_{3}^{0 *}= & \sqrt{\frac{1}{35}}(A-3 B+3 C-D) .
\end{array}
$$

The $\Omega^{-}$vector polarization (closely related to $t_{1}^{0 *}$ ) is, for example,

$$
P=A+\frac{B}{3}-\frac{C}{3}-D \text {. }
$$




\begin{tabular}{c|c|c|c}
\hline Hyperon & Produced by & Exchange & Expected Polarization \\
\hline$\Xi^{-}$ & $\mathrm{p}$ & quark & $\frac{1}{9}(d-3 \epsilon-6 \delta)$ \\
\cline { 2 - 4 } & $\mathrm{n}$ & quark & $-\frac{1}{9}(2 d+3 \epsilon+6 \delta)$ \\
\cline { 2 - 4 } & $\Lambda$ & diquark & $\frac{1}{6}(-4 \epsilon+\delta+d)$ \\
\cline { 2 - 4 } & $\Xi^{0}$ & diquark & $\frac{1}{3}(\epsilon+2 \delta+2 d)$ \\
\cline { 2 - 4 } & $\Sigma^{-}$ & diquark & $\frac{1}{40}(-40 \epsilon+\delta+2 d)$ \\
\hline$\Omega^{-}$ & $\mathrm{p}$ & none & 0 \\
\cline { 2 - 4 } & $\mathrm{n}$ & none & 0 \\
\cline { 2 - 4 } & $\Lambda$ & quark & $\frac{5}{6}(d+\epsilon-\delta)$ \\
\cline { 2 - 4 } & $\Xi^{0}$ & diquark & $\frac{5}{6}(d-\epsilon+\delta)$ \\
\cline { 2 - 4 } & $\Sigma^{-}$ & quark & $-\frac{5}{18} d+\frac{5}{6}(\epsilon-\delta)$ \\
\cline { 2 - 4 } & $\Xi^{-}$ & diquark & $\frac{5}{6}(d-\epsilon+\delta)$ \\
\hline
\end{tabular}

Table B.2: Polarization expectations of the D-M Model. The parameters $\epsilon$ and $\delta$ are roughly 0.10 to 0.30 . The incoming beam polarization is $\mathrm{d}$. These estimates are given to first order in all of the parameters.

Calculating the quark wavefunction overlap between the incoming polarized beam particles and the resulting final state baryons of a given spin projection and applying the relations B.7 describing the polarizations in terms of the spin projections allows prediction of polarizations of any rank. For production at a targeting angle, application of the general rule of (D-M) that sea quarks tend to recombine with their spins down and valence quarks tend to recombine with their spins up in the scattering plane allows the prediction of baryon polarization of any rank from any given beam particle in a similar calculation.

DeGrand and Mietinnen predicted [42] that $\Omega^{-}$produced with a polarized hyperon beam, such as was used in Phase 2 would be vector polarized where the $\Omega^{-}$polarization is $5 / 6$ of the neutral hyperon polarization. Table B.1 summarizes their results. For example, $\Omega^{-}$s produced by $\Lambda$ s have a polarization $\frac{5}{6}(d+\epsilon+\delta)$. This means that if the $\Lambda$ beam is unpolarized and is targeted at an angle, the resulting $\Omega^{-} \mathrm{s}$ will be polarized an amount $5 / 6(\epsilon-\delta)$. If a polarized $\Lambda$ beam is directed at $0 \mathrm{mR}$, as in Phase 2 , the resulting $\Omega^{-} \mathrm{s}$ will have a polarization $5 / 6$ that of the $\Lambda \mathrm{s}$.

As for the higher rank $\Omega^{-}$polarizations, the overlap of the quark wavefunctions 
predicts that the targeting scheme used by E756 produces no rank-2 or rank-3 polarization. A small rank-2 polarization is expected from producing $\Omega^{-} \mathrm{s}$ with a polarized neutral or charged hyperon beam set at a targeting angle. The predictions for rank-2 polarizations are all on the order of $\epsilon$ or $\delta$ times the polarization of the hyperon beam. The predictions for rank-3 are effectively all zero for all targeting conditions and for Phase 2 are identically zero.

\section{B.5 Phase 2 Polarization Predictions}

All of the ingredients of the model have been presented. This section describes how they are put together in a model for the polarization of $\Omega^{-}$and $\Xi^{-}$in Phase 2.

The polarization of the $\Xi^{-} s$ or $\Omega^{-} s$ as a function of momentum is given by

$\mathcal{P}\left(p_{B}\right)=\left(\sum_{n} \int_{p_{n}=p_{B}}^{800}\left(E \frac{d^{3} \sigma}{d p^{3}}\right)_{n} p_{n}\left(E \frac{d^{3} \sigma}{d p^{3}}\right)_{B} p_{B}\left(\Delta p_{B}\right) L_{n} \mathcal{P}_{n} T_{n} \delta\left(x_{n} x_{B}-\frac{p_{B}}{p_{n}}\right) d p_{n}\right) / \sum_{n} N_{B}(p)$,

where $\mathcal{P}_{n}$ is the polarization of the neutral beam (as discussed in Section B.2) and $T_{n}$ is the polarization transfer from the neutral species to species B (as discussed in Section B.4).

The integral, Equation B.9, was performed numerically. The polarization of the $\Xi^{-}$ as a function of $P_{\Xi-}$ are shown in Figure B.5. The $\Omega^{-}$polarization is also shown in Figure B.5. Both the $\Xi^{-}$and $\Omega^{-}$polarizations are predicted to increase with momentum.

\section{B.6 Discussion}

There are two ways in which the prediction does not match the results. First, the $\Xi^{-}$ polarization prediction is too small. Second, a feature obvious from the plots is that the polarization extrapolates towards a substantial amount at zero momentum.

Possible explanations for the discrepancy in the overall size of the $\Xi^{-}$polarization include 


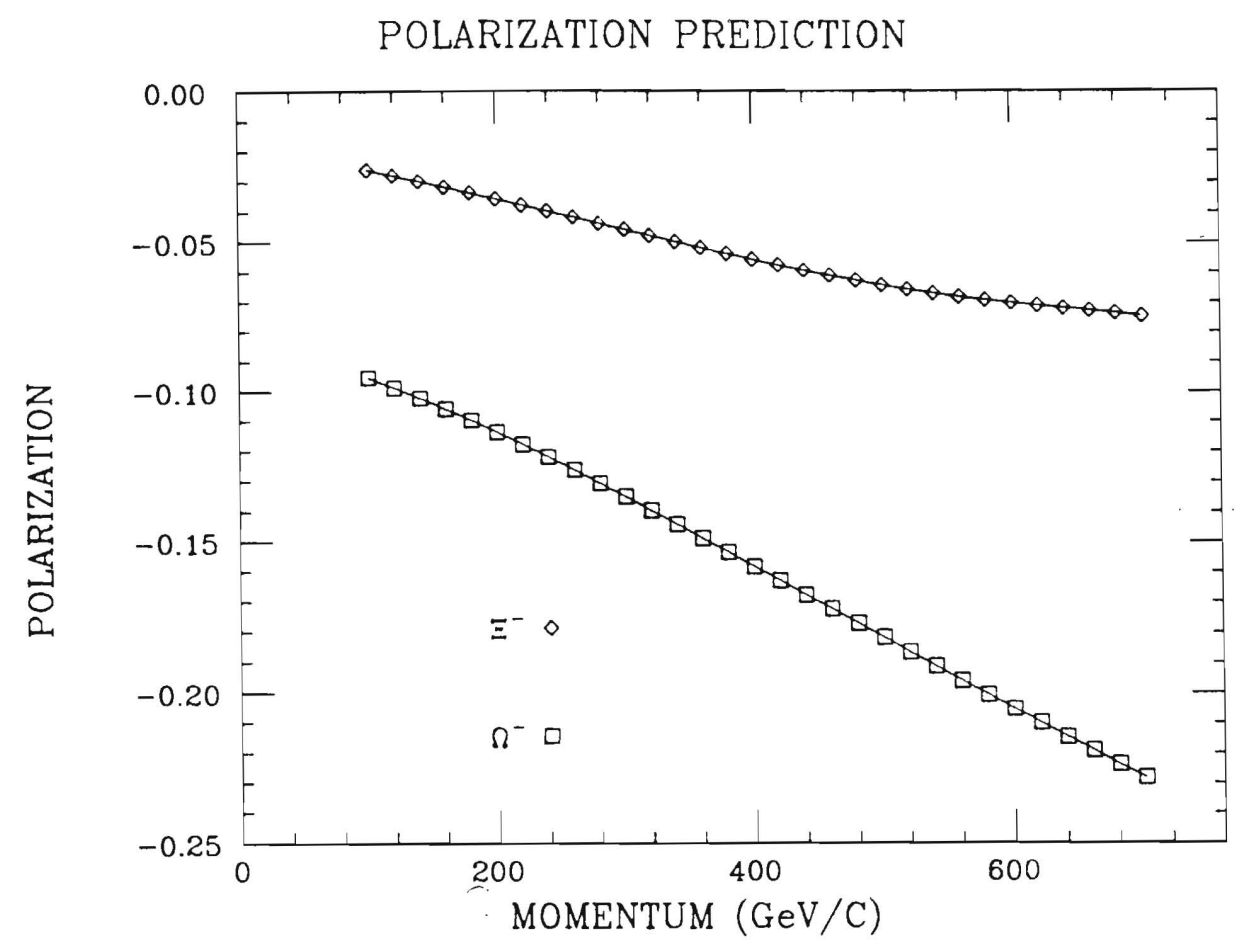

Figure B.5: Predictions for Phase $2 \Omega^{-}$and $\Xi^{-}$polarization.

1. the spin transfer of the $\Lambda$ polarization to the $\Xi^{-}$is closer to $1 / 2$ than $1 / 6$,

2. the $\Xi^{0} \rightarrow \Xi^{-}$cross section is a factor of 3 higher, or

3. some combination of the above.

The spin transfer from $\Lambda \mathrm{s}$ to $\Xi^{-} \mathrm{s}$ given in Table B.2 proceeded through exchange of a diquark. If instead, a single strange quark was exchanged, the spin transfer would be $7 / 11$ instead of $1 / 6$.

There is an alternative to the simple parameterization for spin transfer which could explain the trend at low momentum. It is: the smallest exchange which produces the $\Omega^{-}$preserves the most polarization. A harder scattering preserves less. Thus, the polarization transfer could be parameterized as $\mathcal{P}_{\Omega}=\mathcal{P}_{0} x_{\Omega}$, where $x_{n}$ is the fraction of the neutral particle momentum carried by the $\Omega^{-}$and $\mathcal{P}_{0}$ is the spin transfer coefficient from Table B.4. 


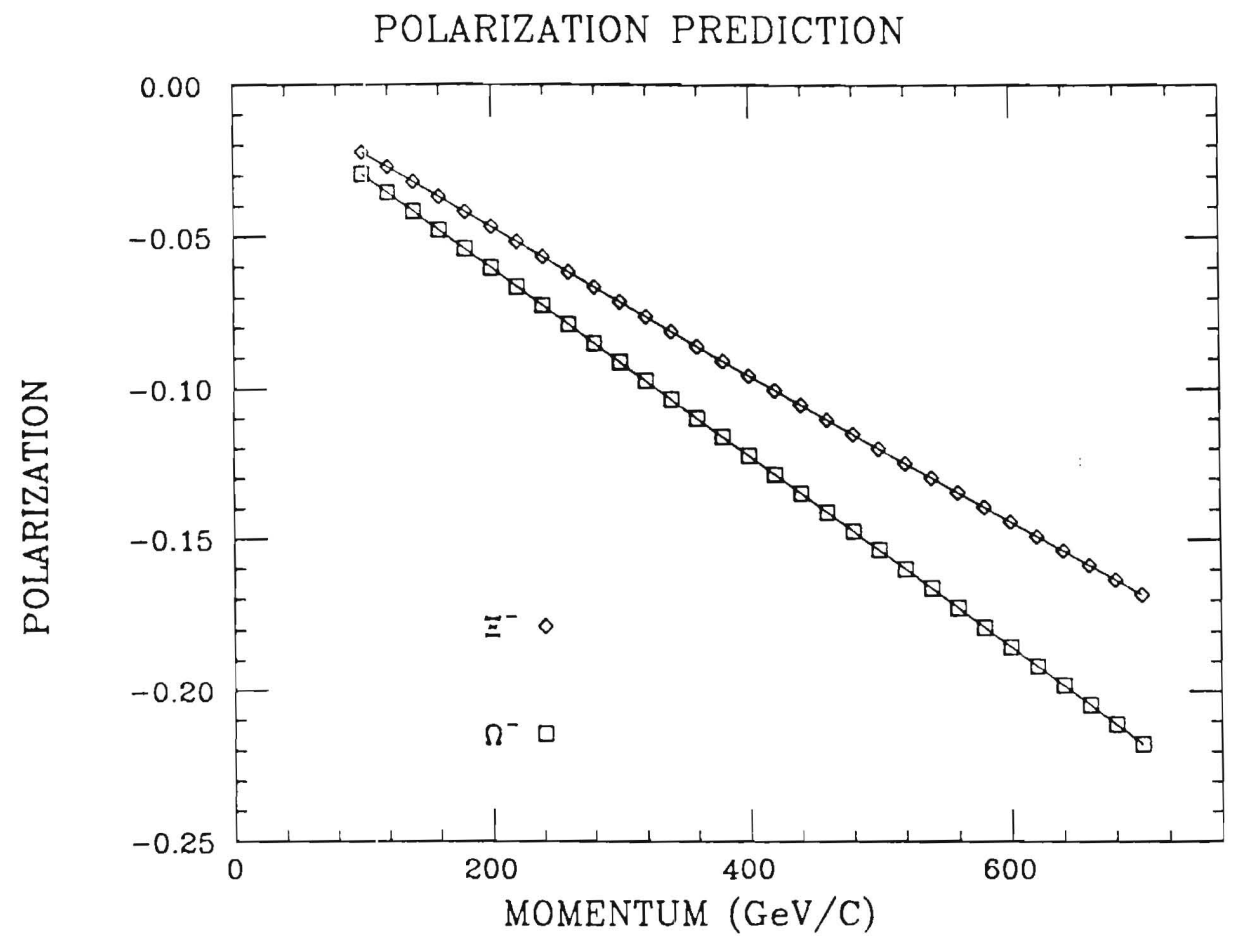

Figure B.6: Modified predictions for Phase $2 \Omega^{-}$and $\Xi^{-}$polarization.

The polarization predictions with the two suggested modifications are shown in Figure B.6. The agreement, while not perfect, is substantially better.

Finally, this model is based on improvisation. However, it suggests that a measurement of the content and polarization of the neutral beam is an interesting experiment. It provides some clues to future experiments measuring $\mu_{\Omega^{-}}$with this technique. And it makes some suggestions regarding the dynamics of spin transfer. 


\section{Appendix C \\ The Rutgers $1 \mathrm{~mm}$ Pitch MWPCs}

MWPCs C2 and C3 of Figure 3.3 were built by Rutgers for E756. They are noteworthy because they were large MWPCs $\left(650 \mathrm{~cm}^{2}\right)$ considering their small pitch $(1 \mathrm{~mm})$. Sauli's review paper [55] claims $1 \mathrm{~mm}$ pitch MWPCs of more than $100 \mathrm{~cm}^{2}$ are difficult to operate. This difficulty was not observed. This Appendix outlines their design and performance. Design modifications for larger MWPCs with small pitch are suggested.

Their design was based on a University of Wisconsin model of $1 / 4$ the active area. It was the "standard design" of three cathode (high-voltage) planes sandwiching two anode (sense-wire) planes. The design characteristics were:

$\begin{array}{cl}\text { Wire supporting frame } & 3 / 16 \text { inch thick Laminated FR }-4 \text { (fiberglass) } \\ \text { Half - gap } & 3 / 16 \text { inch } \\ \text { Active area } & 100 \mathrm{in}^{2}\end{array}$

$\begin{array}{cl}\text { Cathode wires (512) } & 2 \text { mil Be }-\mathrm{Cu} \text { alloy 25 } \\ \text { Cathode wire tension } & 100 \text { grams - weight equivalent } \\ \text { Cathode wire spacing } & 1 / 2 \mathrm{~mm} \\ \text { Anode wires (256) } & 0.8 \mathrm{mil} \text { Gold - plated Tungsten } \\ \text { Anode wire tension } & 50 \text { grams - weight equivalent } \\ \text { Anode wire spacing } & 1 \mathrm{~mm}\end{array}$

The sense planes were wound at Fermilab's Lab 6 on a digital high precision windingmachine normally used for making mini-drift chambers. The wires were wound onto a transfer frame. The transfer frame was then clamped onto the planes. This held the wires tension during the soldering process. The solder pads were $5 / 8$ inch long and 
$2 / 3 \mathrm{~mm}$ wide. A thin ( 1/8 inch wide) mylar strip with a layer of two-component epoxy fixed the wires at the edge of the active area during the soldering process. This insured that the wires would not be pushed out of alignment during soldering. The high-voltage planes were wound on Lab 6 standard MWPC winders. The fiberglass planes were mounted on a rolled steel frame fitted with two dowel pins to control their relative alignment.

The chamber gas was the argon-freon-methylal combination mentioned in Chapter 3. It consisted of $99.88 \%$ argon and $0.12 \%$ Freon $13-\mathrm{B} 1$ by weight bubbled through a jar of liquid methylal at 2 degrees centigrade. Gas inlet and exhaust ports were cut in the steel frame and bottom fiberglass plane. The gas was held at atmospheric pressure in the chamber. The MWPCs operating voltage was -4150 volts. At operating voltage, the MWPCs had a 4-6 mV signal at the passage of a charged particle. 1/16 inch diameter Buna o-rings were located in grooves between the planes. The windows were $1 / 2$-mil mylar sheets.

The chambers were operated in a beam which occasionally exceeded $15 \mathrm{khz} /$ wire at their locations. Their efficiency was shown in Table 3.3. One chamber (C2) had a high efficiency $(>97 \%$ ) for both planes. The other (C3) had one plane which was $\approx 95 \%$ efficient and one plane which was $\approx 25 \%$ efficient. The inefficient plane was due to one of it's half-gaps being larger than the others in the MWPC. This plane operated at a higher field which could not be reached without causing breakdown at the other anode. A solution to this problem implemented after the run was to use four high-voltage planes, two surrounding each anode plane, with a grounded plane in the middle to isolate the two views.

The size limit for a wire chamber of this design due to the electrostatic repulsion of the wires exceeding the restoring force of the wire tension is about $75 \times 75 \mathrm{~cm}^{2}$. However, a chamber of this size would break a wire at every spark due to the large capacitance of the planes. In order to build a $1 \mathrm{~mm}$ chamber with a size much larger than those used in E756, it is necessary to use a smaller half-gap (1/8 inch is recommended) in order to decrease the operating voltage. Care must be maintained to insure that the 
half gap spacing is uniform between all of the planes.

In summary, a design for large $1 \mathrm{~mm}$ pitch MWPCs has been tested and proved to work in an experiment. Improvements have been suggested which allow the possible construction of even larger $1 \mathrm{~mm}$ pitch MWPCs. 


\section{References}

[1] G. Bunce et al. Phys. Rev. Lett., 36:1113, 1976.

[2] L.G.Pondrum. Phys. Rep., 122:, 1985.

[3] R. Rameika et al. Phys. Rev. D, 33:3172, 1986.

[4] K. B. Luk et al. Phys. Rev. D, 38:19, 1988.

[5] Carol Ann Wilkinson. The Polarization and Magnetic Moment of the Sigma Plus Hyperon. PhD thesis, University of Wisconsin - Madison, 1985. (unpublished).

[6] S. A. Gourlay et al. Phys. Rev. Lett, 56:2244, 1986.

[7] J. Bensinger et al. Phys. Rev. Lett., 50:313, 1983.

[8] BIS-2 Collaboration. Sov. J. Nucl. Phys., 43:395, 1986.

[9] Particle Data Group. Review of particle properties. Phys. Lett., 204B:1, 1988.

[10] L. H. Trost et al. Phys. Rev. D, 40:1703, 1988.

[11] R. Rameika et al. Phys. Rev. Lett., 52:581, 1984.

[12] V. E. Barnes et al. Phys. Rev. Lett., 12:204, 1964.

[13] J. Finjord and M. K. Gaillard. Phys. Rev. D, 22:778, 1980.

[14] Dan-di Wu and J. Rosner. Phys. Rev. D, 33:1367, 1986.

[15] D. Tadić H. Galić and J. Trampetić. Phys. Lett., 89B:249, 1980.

[16] Lee Brekke and Robert G. Sachs. Phys. Rev. D, 28:1178, 1983. 
[17] Lee Brekke and Jonathan L. Rosner. Enrico Fermi Institute Preprint EFI87-80, $:, 1987$.

[18] Howard Georgi and Aneesh Monoher. Phys. Lett., 132B:183, 1983.

[19] D. B. Lichtenberg. Z. Phys. C, 7:143-148, 1981.

[20] C. B. Chiu et al. Phys. Rev. D, 33:1961, 1986.

[21] Nathan Isgur and Gabriel Karl. Phys. Rev. D, 21:3175, 1980.

[22] S. Perantonis. Phys. Rev. D, 37:2687, 1988.

[23] Yukio Tomowaza. Phys. Rev. D, 19:1626, 1979.

[24] R. C. Verma and M. P. Khanna. Phys. Lett., 183B:207, 1981.

[25] T. Das and S. P. Misra. Phys. Lett., 96B:165, 1980.

[26] A. Chodos et al. Phys. Rev. D, 10:2599, 1974.

[27] T. DeGrand et al. Phys. Rev. D, 12:2060, 1975.

[28] K. Ushio. Phys. Lett., 158B:71, 1985.

[29] M. Krivoruchenko et al. Moscow-ITEP 89-23, :, 1989.

[30] K. Tsushima et al. Phys. Lett., 205B:128, 1988.

[31] Ted Barnes. Nucl. Phys., B96:353-364, 1975.

[32] J. Cohen and H. J. Weber. Phys. Lett., 165B:229, 1985.

[33] N. Barik and B. K. Dash. Phys. Rev. D, 31:31, 1985.

[34] Z. Dziembowski and L. Mankiewicz. Phys. Rev. Lett., 55:1839, 1985.

[35] W. Y. P. Hwang. Z. Phys. C, 16:327-330, 1983.

[36] V. P. Efrosinin and D. A. Zaikin. Sov. J. Nucl. Phys., 44:681, 1986. 
[37] C. Bernard et al. Phys. Rev. Lett., 49:1076, 1982.

[38] B. Andersson G. Gustafson and G. Ingelman. Phys. Lett., 86B:417, 1979.

[39] T. DeGrand and H. I. Miettinen. Phys. Rev. D, 24:2419, 1981. This paper contains an error in the sign of the Sigma Minus polarization which was corrected in Reference 42.

[40] J. Szwed. Phys. Lett., 105B:403, 1981.

[41] Lars Skold. Physica Sicripta, 31:464, 1985.

[42] Thomas A. Degrand et al. Phys. Rev. D, 32:2445, 1985.

[43] G. Bunce et al. Phys. Lett., 86B:386, 1979.

[44] Kam-Biu Luk. A Study of The Omega Minus Hyperon. PhD thesis, Rutgers, The State University of New Jersey, 1983. (unpublished).

[45] H. T. Diehl et al. Preliminary $\Xi^{-}$and $\Omega^{-}$polarization and magnetic moment results. In K. Haller, editor, Proceedings of the Storrs Meeting of the Division of Particles and Fields of the American Physical Society, 1989.

[46] G. Bunce et al. Nucl. Instr. and Meth., 172:553, 1980.

[47] Jeffrey Walton Duryea. A Precision Measurement of the $\Xi^{-}$Polarization and Magnetic Moment. PhD thesis, University of Minnesota, 199? (This thesis is in progress at this time.).

[48] M. I. Shirkov. JETP, 6:748, 1958.

[49] A. Simon and T. A. Welton. Phys. Rev., 90:1036, 1953.

[50] K. Abe et al. Phys. Rev. D, 32:2869, 1985.

[51] L. W. Jones et al. In S. N. Ganguli et al., editor, Proceedings of the Xth International Symposium on Multiparticle Dynamics, 1979. 
[52] A. Beretvas et al. Phys. Rev. D, 34:53, 1986.

[53] Byron Gene Lundberg. Neutral Strange Particle Production and Polarization at Large $P_{T}$. PhD thesis, University of Wisconsin - Madison, 1984. (unpublished).

[54] Kam-Biu Luk. Fermilab Preprint 89-40, :, 1989.

[55] F. Sauli. Cern Preprint 77-09, :53, 1977. 


\section{Vita}

\section{Herman Thomas Diehl, III}

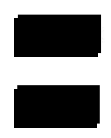

1978-82

1982

1982

1983

1983-90

1983-85

1985-90 Research Assistant, Department of Physics, Rutgers, The State University of New Jersey, New Brunswick, New Jersey.

1987

1988

1988

1989

1990

1990

Physics major, Bates College, Lewiston, Maine.

B.A., Bates College.

Semiconductor Research and Development Lab, Motorola Co. Pheonix, Az.

S. R. Wilson et al. "Characterization of Ion Implanted Silicon Annealed with a Graphite Radiation Source", IEEE Transactions on Nuclear Science, NS-30:1734, (1983).

Graduate reseas:ch in Physics, Rutgers, The State University of New Jersey, New Brunswick, New Jersey.

Teaching Assistant, Department of Physics, Rutgers, The State University of New Jersey, New Brunswick, New Jersey.

N. Grossman et al. "Measurement of the Lifetime of $\mathrm{K}_{s}^{0}$ Mesons in the Momentum range 100 to $350 \mathrm{GeV} / \mathrm{c}$ ", Phys. Rev. Lett., $\underline{59}$ :18, (1987).

5th NATO Advanced Study Institute on Techniques and Concepts of High Energy Physics, Christiansted, United States Virgin Islands.

H. T. Diehl et al. "Preliminary $\Xi^{-}$and $\Omega^{-}$Polarization and Magnetic Moment Results", Paper presented at the 1988 Meeting of the Division of Particles and Fields of the American Physical Society, Storrs, Connecticut.

S. Teige et al. "Measurement of the $\Xi^{0} \rightarrow \Sigma^{0} \gamma$ Branching Ratio and Asymmetry Parameter", Phys. Rev. Lett., 63: 2717, (1989).

H. T. Diehl et al. "Measurement of the Polarization and Magnetic Moment of the $\Omega^{-}$Hyperon", Paper presented at the 1990 Meeting of the Division of Particles and Fields of the American Physical Society, Houston, Texas.

Ph.D. in Physics. 